\title{
Insight in disease and drug interactions, with treatment optimisation of patients with cancer
}

Citation for published version (APA):

Knapen, L. (2019). Insight in disease and drug interactions, with treatment optimisation of patients with cancer. [Doctoral Thesis, Maastricht University]. Gildeprint Drukkerijen.

https://doi.org/10.26481/dis.20190510lk

Document status and date:

Published: 01/01/2019

DOI:

10.26481/dis.20190510lk

Document Version:

Publisher's PDF, also known as Version of record

\section{Please check the document version of this publication:}

- A submitted manuscript is the version of the article upon submission and before peer-review. There can be important differences between the submitted version and the official published version of record.

People interested in the research are advised to contact the author for the final version of the publication, or visit the DOI to the publisher's website.

- The final author version and the galley proof are versions of the publication after peer review.

- The final published version features the final layout of the paper including the volume, issue and page numbers.

Link to publication

\footnotetext{
General rights rights.

- You may freely distribute the URL identifying the publication in the public portal. please follow below link for the End User Agreement:

www.umlib.nl/taverne-license

Take down policy

If you believe that this document breaches copyright please contact us at:

repository@maastrichtuniversity.nl

providing details and we will investigate your claim.
}

Copyright and moral rights for the publications made accessible in the public portal are retained by the authors and/or other copyright owners and it is a condition of accessing publications that users recognise and abide by the legal requirements associated with these

- Users may download and print one copy of any publication from the public portal for the purpose of private study or research.

- You may not further distribute the material or use it for any profit-making activity or commercial gain

If the publication is distributed under the terms of Article $25 \mathrm{fa}$ of the Dutch Copyright Act, indicated by the "Taverne" license above, 
Insight in disease and drug interactions, with treatment optimisation of patients with cancer 
(C) Lotte Knapen, Maastricht 2019, All rights reserved

Cover: Tjeerd Bosma (tjeerd@mintis.nl)

Layout: Tiny Wouters Lenssen

Printing: Gildeprint B.V.

ISBN: 978-94-6323-576-1

Printing of this thesis was kindly supported by: het Nederlands Bijwerkingen Fonds. 


\title{
Insight in disease and drug interactions, with treatment optimisation of patients with cancer
}

\author{
PROEFSCHRIFT \\ ter verkrijging van de graad van doctor aan de Universiteit Maastricht, \\ op gezag van de Rector Magnificus, prof. dr. Rianne M. Letschert, \\ volgens het besluit van het College van Decanen, \\ in het openbaar te verdedigen \\ op vrijdag 10 mei 2019 om 14.00 uur
}

door

Lotte Marieke Knapen 


\section{Promotor}

Prof. dr. V.C.G. Tjan-Heijnen

\section{Copromotoren}

Dr. S. Croes

Dr. F. de Vries

Dr. N.P. van Erp (Radboudumc)

\section{Beoordelingscommissie}

Prof. dr. N.C. Schaper (voorzitter)

Dr. M. Aarts

Prof. dr. H.-J. Guchelaar (Universiteit Leiden)

Prof. dr. R. Mathijssen (Erasmus Universiteit) 


\section{Contents}

Chapter 1 Introduction and outline of the thesis

Chapter 2 The impact of medications on cancer risk in real-life populations

2.1 Use of biguanides and the risk of colorectal cancer: a registerbased cohort study Curr Drug Saf. 2013;8(5):349-56

2.2 Use of incretin agents and risk of pancreatic cancer: a population-based cohort study Diabetes Obes Metab. 2016;18(3):258-65

Chapter 3 Effectiveness of targeted oncologic therapies in real-life populations

3.1 A real-life study on the implementation and effectiveness of exemestane plus everolimus per hospital type in patients with advanced breast cancer. A study of the Southeast Netherlands advanced breast cancer registry

Breast. 2019;44:46-51 on the effectiveness of vemurafenib in metastatic BRAF V600 mutated melanoma: a retrospective cohort study Target Oncol. 2018:13(3):363-370

Chapter 4 Optimisation of treatment in real-life individual cancer patients

4.1 Development and validation of an analytical method using UPLC-MS/MS to quantify everolimus in dried blood spots in the oncology setting J Pharm Biomed Anal. 2018;149:106-113

4.2 Clinical validation study of dried blood spot for determining everolimus concentration in patients with cancer Eur J Clin Pharmacol. 2018;74(4):465-471 erlotinib, and sunitinib in routine clinical outpatient cancer care Ther Drug Monit. 2014;36(3):326-34 
Chapter 5 Summary, general conclusions and future perspectives

$\begin{array}{lll}\text { Appendices } & \text { List of abbreviations } & 159\end{array}$

Nederlandse samenvatting $\quad 165$

Valorisation 173

List of publications $\quad 179$

Dankwoord 181

Curriculum vitae $\quad 183$ 

0

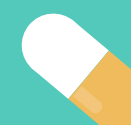

$\theta$

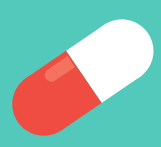

$\theta$

○ 


\section{Chapter 1}

Introduction and outline of the thesis 


\section{Introduction and outline of the thesis}

\section{Cancer}

Cancer is a major threat to human health, affecting burden of morbidity and leading to mortality of millions of people worldwide. Of the 56.9 million deaths worldwide in 2015, 8.8 million were attributed to the death of people from cancer ${ }^{1}$. Cancer is characterised by an uncontrolled division of cells which can invade local tissue and metastasise to other organs. During the last years, substantial progress has been made in the fight against cancer. Cancer can be detected earlier and patients can be treated with better treatment ${ }^{1}$. These improvements resulted in an increased likelihood of long-term survival ${ }^{2}$. The longer survival is shown by the lifetime risk to develop cancer of $38 \%$ in females and $40 \%$ in males versus the lifetime risk to die from cancer of $19 \%$ in females and $22 \%$ in males ${ }^{3}$. This illustrates that patients currently frequently die from other causes than cancer, potentially due to better treatment options ${ }^{1,2}$. Hence, patients with cancer are often exposed to multiple coexisting diseases, making health care delivery even more complex.

\section{Comorbidity and medications for comorbidities versus cancer risk}

Comorbidities are coexisting diseases and conditions present at the time of diagnosis that influence the overall health and survival. The prevalence of any comorbidities in the period of 2002-2008 among the general Dutch population was high (34\%), and was even higher among patients with cancer of 55 years and older $(73 \%)^{4}$. The high number of multimorbidities among patients with cancer may not come as a surprise, since comorbidities and cancer share many risk factors, such as obesity, low physical activity, alcohol consumption and smoking ${ }^{5}$. As a consequence, unhealthy lifestyle factors are major contributors to the global burden of cancer. For example, body fatness (both overweight and obesity versus healthy weight) contributes for $13 \%$ to the 10 -year burden of cancers ${ }^{6}$. Further, smoking (current and former smokers versus never smokers), alcohol consumption ( $>2$ drinks/day versus $\leq 2$ drinks/day) and simultaneous smoking and more than 2 drinks of alcohol per day are factors that contribute for respectively $13 \%, 6 \%$ and $29 \%$ to the 10 -year burden of cancers ${ }^{6}$.

Furthermore, besides the fact that patients with cancer may have multiple comorbidities, these comorbidities may also impact the predisposition to cancer. For example, type 2 diabetes mellitus (T2DM) itself has been associated with various types of cancers including colorectal, pancreatic, liver, endometrium, rectum, breast, and bladder cancer ${ }^{7-9}$.

In addition, the medications used to treat the comorbidities may either in- or decrease the predisposition to cancer. To illustrate, T2DM patients treated with biguanides may have a decreased risk of colorectal cancer ${ }^{10}$, while T2DM patients 
treated with incretin agents may have an increased risk of pancreatic cancer ${ }^{11}$. Last, comorbidities may also affect the timing of the cancer diagnosis, the treatment options and compliance.

Therefore, gaining knowledge on the interaction between cancer on one hand and non-cancer diseases and treatments on the other hand is of utmost importance. Yet, it is difficult to investigate the impact of drug use for non-cancer diseases on the risk to develop cancer in real-life populations, due to the potential influence of the non-cancer disease itself on the risk to develop cancer. Thus, results of (observational) studies are often conflicting and inconclusive.

\section{Efficacy versus effectiveness of cancer treatment}

Most patients are being treated for their condition (i.e. cancer and/or comorbidities) according to evidence-based treatment guidelines which are based on results and conclusions drawn from randomised clinical trials (RCTs). The design of these trials is focused on providing causal conclusions regarding the efficacy and safety of new (anticancer) drugs. It is therefore that RCTs are known for their high internal validity $^{12,13}$. However, the high internal validity comes at the cost of limited external validity.

Real-life populations represent a more naturalistic environment as compared with patients participating in RCTs, since real-life patients are exposed to many factors that are being controlled for in the RCTs. For example, RCTs are conducted under ideal conditions, require strict adherence to structured protocols and have restrictive in- and exclusion criteria $^{12}$. Patients with comorbidities can therefore often not participate in RCTs. As a consequence, it is estimated that the representativeness of real-life patients compared with patients participating in RCTs is only $29 \%{ }^{12}$, whereas cancer treatment may be less effective in real-life patients as compared with patients participating in RCTs.

Real-life studies in oncology populations provide additional insight for the everyday clinical practice regarding effectiveness of cancer treatment (degree and velocity of implementation, characteristics of patients actually selected for treatment, data on progression-free and overall survival) and its safety (toxicity profile of the treatment, delivered dose modifications, drug-drug interactions between anticancer and non-cancer drugs). This real-life information should be incorporated when developing evidence-based guidelines to make more accurate treatment decisions for future patients. As an example, the latest international guideline on primary breast cancer provides advice to consider trastuzumab-based adjuvant therapy for patients with small, node-negative, HER2-postitive tumours ${ }^{14,15}$. Yet, real-life studies on implementation and effectiveness of new drugs, and the interaction between the use of anticancer and non-cancer drugs in real-life is often poorly investigated, specifically shortly after approval of the new anticancer drug. 


\section{Optimisation of treatment}

Cancer treatment increasingly becomes personalised, amongst others by incorporating relevant unique genetic and molecular abnormalities of the tumour $^{16}$. Well known examples of tumour characteristics that affect the (targeted) treatment given include the presence of the estrogen and/or progesterone-receptor in breast cancer (e.g. exemestane plus everolimus) ${ }^{17}$, the BRAF V600 gene mutation in metastatic melanoma (e.g. vemurafenib) ${ }^{18}$, the presence of the BCR-ABL fusion gene in chronic myeloid leukemia (e.g. imatinib) ${ }^{19}$, or abnormalities in C-KIT in gastrointestinal stromal tumour (e.g. imatinib) ${ }^{20}$. Tyrosine kinase inhibitors (TKIs) are small molecule therapeutic agents that specifically target the molecular alterations of cancer cells (e.g. tumour characteristics).

In addition to targeting treatment specifically for the patients' tumour characteristics, it is possible to optimise and personalise the treatment through the use of therapeutic drug monitoring (TDM) in patients with cancer ${ }^{21-23}$. TDM comprises the measurement and interpretation of drug concentrations in biological fluids to support the determination of drug dosage for the individual patient ${ }^{24}$. Many of the targeted oral oncolytics used to treat patients with cancer can be characterised by the steep exposure-response relationships and a narrow therapeutic window ${ }^{21-23}$. In addition, interpatient variability in pharmacokinetics is often large ${ }^{21-23}$. Yet, most of the TKIs are fixed-flat dosed according to the 'one-size-fits-all' approach and are orally administrated, which may result in varying drug exposure and response levels among patients with cancer ${ }^{21-23}$. For this reason the use of TDM in daily clinical practice may be promising for the (near) future. However, at present there are various obstacles that prevent TDM from being routinely used in daily practice. The most important obstacle to overcome the implementation of TDM is the lack of evidence from prospective RCTs that have demonstrated considerable better treatment outcomes with the use of pharmacokinetically guided dosing versus fixed-flat dosing ${ }^{21,25}$. Other obstacles include the invasive nature of TDM and the suboptimal timing of venous sampling. Dried blood spot (DBS) sampling is an attractive alternative to venous sampling, since patients can perform sampling at home and send it in by mail to the clinical lab a couple of days before their routine visit to the outpatient clinic ${ }^{26}$.

Hence, we hypothesised that individualised dosing instead of fixed-flat dosing of TKIs might optimise treatment outcome and that this might be supported by performing TDM, using DBS sampling to improve feasibility. It is therefore of interest to investigate whether DBS concentrations correlate with whole-blood concentrations and to evaluate determinants of subtherapeutic plasma concentrations of real-life individual cancer patients treated with TKIs. 
This thesis is designed to fill in a few of the aforementioned 'gaps'. The drugs chosen as proof of concept in this thesis are the antidiabetic biguanides and incretin agents and the anticancer drugs everolimus, vemurafenib, imatinib, erlotinib, and sunitinib.

\section{Specific objectives of this thesis}

This thesis consists of three main parts: 1 ) to determine the association between the use of antidiabetic drugs and risk of cancer in real-life populations; 2) to evaluate the implementation and effectiveness of exemestane plus everolimus and of vemurafenib in real-life oncology populations and to assess whether vemurafenib effectiveness was impacted by the potential interaction between acid reducing agents and vemurafenib; and 3) to improve individualised dosing by developing a bioanalytical assay, clinically validating the assay and by assessing the feasibility to perform TDM in real-life patients with cancer. See also Figure 1.1.

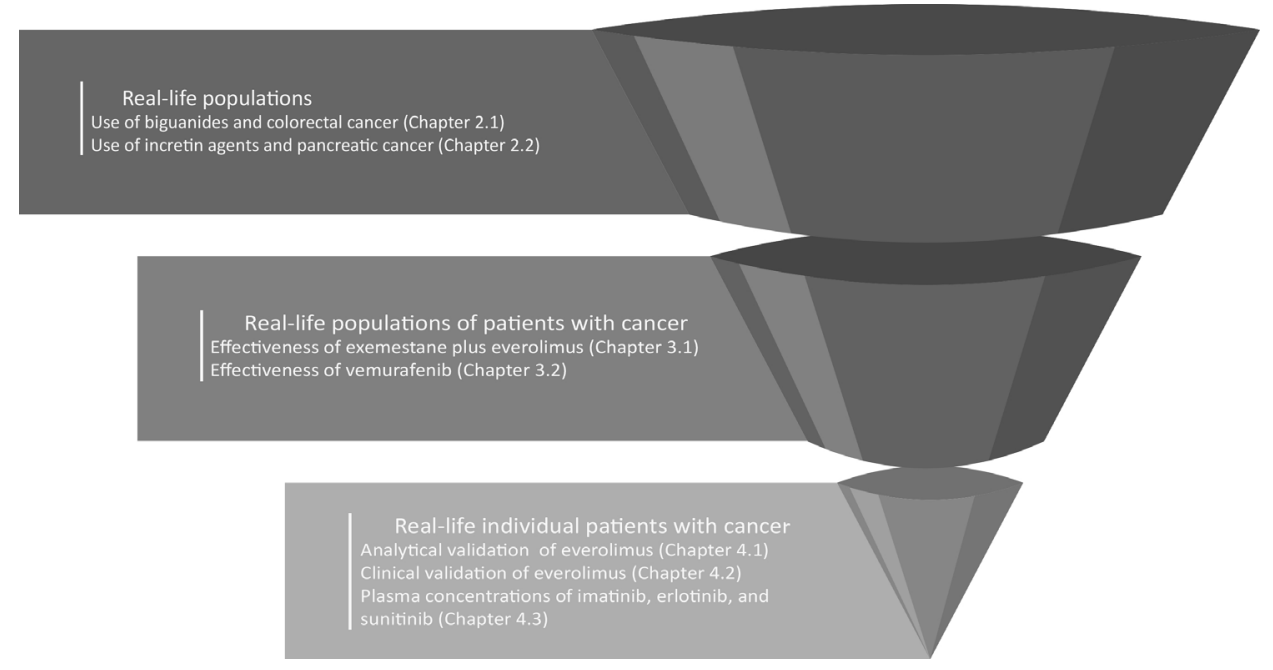

Figure 1.1 Schematic outline of this thesis. 


\section{Outline}

Chapter 2 consists of two cohort studies that assessed the risk of cancer with the use of antidiabetic drugs. In Chapter $\mathbf{2 . 1}$ we investigated the association between the use of biguanides and the risk of colorectal cancer using the Danish National databases $(n=5.5$ million) ${ }^{27}$. In Chapter $\mathbf{2 . 2}$ we evaluated the association between the use of incretin agents and the risk of pancreatic cancer using the Clinical Practice Research Database (CPRD) from the United Kingdom ( $n=13$ million $)^{28}$.

Chapter 3 consists of two observational cohort studies that evaluated the effectiveness of anticancer treatment, taking treatment dose, duration and drug-drug interactions into account. In Chapter 3.1 we used real-life data from the SOutheast Netherlands Advanced BREast cancer (SONABRE) registry to assess the implementation and effectiveness of exemestane plus everolimus treatment per hospital type in patients with hormone receptro-positive/human epidermal growth factor receptor 2-negative (HR-positive/HER2-negative) advanced breast cancer. In Chapter 3.2 the impact of vemurafenib dose and simultaneous use of vemurafenib and acid-reducing agents (ARAs) on the risk of progression in metastatic BRAF V600 mutated melanoma patients was assessed. Although the interaction between ARAs and various TKIs (e.g. erlotinib) was known, this information was lacking for vemurafenib ${ }^{29,30}$. Furthermore, the impact of a reduced vemurafenib dose versus full-dose vemurafenib on progression-free survival in melanoma patients was unknown. Data were obtained from pharmacy dispensing data and electronic patient records of an academic hospital in the Netherlands $(n=115)$.

Chapter 4 consists of three studies describing the TDM of targeted agents. While TDM of everolimus, including dried blood spot (DBS) sampling, has been routinely performed in solid organ transplantation medicine, it is yet uncommon in the treatment of cancer ${ }^{26,31}$. DBS is a minimal invasive sampling method which can be performed by patients at their homes. Therefore, in Chapter $\mathbf{4 . 1}$ an analytical method using ultra performance liquid chromatography-tandem mass spectrometry (UPLC-MS/MS) to quantify everolimus in DBS in the oncology setting was developed and analytically validated. This method was applied in Chapter 4.2 to assess the level of agreement between everolimus concentrations measured in capillary blood obtained by finger prick dried versus whole-blood obtained by venipuncture in patients with cancer treated with everolimus. Last, we aimed to assess the occurrence of suboptimal plasma concentrations of imatinib, erlotinib, and sunitinib and aimed to investigate the contribution of patient- and medication-related factors on suboptimal plasma concentrations in daily outpatient cancer care. To address this research question we used data from the electronic patient records of a hospital in the Netherlands $(n=108)$. The results of this study are described in Chapter 4.3.

This thesis concludes with a summary, general discussion of the results and prospects for future research. 


\section{References}

1. World Health Organization. Home/News/Fact sheets/Detail/Cancer. Retrieved from http://www.who.int/en/ news-room/factsheets/detail/cancer. Accessed July 2018.

2. Dal Maso L, Guzzinati S, Buzzoni C, et al. Longterm survival, prevalence, and cure of cancer: a population-based estimation for 818902 Italian patients and 26 cancer types. Ann Oncol. 2014;25(11):2251-60.

3. Lifetime risk of developing or dying from cancer. Retrieved from https://www.cancer.org/cancer/ cancerbasics/lifetime-probability-of-developing-ordying-from-cancer.html. Accessed October 2018.

4. van Oostrom SH, Picavet HS, van Gelder BM, et al. Multimorbidity and comorbidity in the Dutch population - data from general practices. BMC Public Health. 2012;12:715.

5. Giovannucci E, Harlan DM, Archer MC, et al. Diabetes and cancer: a consensus report. Diabetes Care. 2010;33(7):1674-85.

6. Arriaga ME, Vajdic CM, Canfell K, et al. The burden of cancer attributable to modifiable risk factors: the Australian cancer-PAF cohort consortium. BMJ Open. 2017;7(6):e016178.

7. Vigneri $P$, Frasca $F$, Sciacca $L$, et al. Diabetes and cancer. Endocr Relat Cancer. 2009;16(4): 1103-23.

8. Huxley R, Ansary-Moghaddam A, Berrington de Gonzalez A, et al. Type-Il diabetes and pancreatic cancer: a meta-analysis of 36 studies. Br J Cancer. 2005;92(11):2076-83.

9. Larsson SC, Orsini N, Wolk A. Diabetes mellitus and risk of colorectal cancer: a meta-analysis. J Natl Cancer Inst. 2005;97(22):1679-87.

10. Zhang ZJ, Zheng ZJ, Kan H, et al. Reduced risk of colorectal cancer with metformin therapy in patients with type 2 diabetes: a metaanalysis. Diabetes Care. 2011;34(10):2323-8.

11. Boniol $M$, Franchi $M$, Bota $M$, et al. IncretinBased Therapies and the Short-term Risk of Pancreatic Cancer: Results From Two Retrospective Cohort Studies. Diabetes Care. 2018;41(2):286-92.

12. Kennedy-Martin T, Curtis S, Faries D, et al. A literature review on the representativeness of randomized controlled trial samples and implications for the external validity of trial results. Trials. 2015;16:495.
13. Van Spall HG, Toren A, Kiss A, et al. Eligibility criteria of randomized controlled trials published in high-impact general medical journals: a systematic sampling review. JAMA 2007;297(11):1233-40.

14. Tolaney SM, Barry WT, Dang CT, et al. Adjuvant paclitaxel and trastuzumab for nodenegative, HER2-positive breast cancer. $\mathrm{N}$ Engl J Med. 2015;372(2):134-41.

15. Senkus E, Kyriakides S, Ohno S, et al. Primary breast cancer: ESMO Clinical Practice Guidelines for diagnosis, treatment and follow-up. Ann Oncol. 2015;26Suppl5:v8-30.

16. Schmidt $\mathrm{KT}$, Chau $\mathrm{CH}$, Price DK, et al. Precision Oncology Medicine: The Clinical Relevance of Patient-Specific Biomarkers Used to Optimize Cancer Treatment. J Clin Pharmacol. 2016;56(12):1484-99.

17. Baselga J, Campone $\mathrm{M}$, Piccart $\mathrm{M}$, et al. Everolimus in postmenopausal hormonereceptor-positive advanced breast cancer. $\mathrm{N}$ Engl J Med. 2012;366(6):520-9.

18. Chapman PB, Hauschild A, Robert C, et al. Improved survival with vemurafenib in melanoma with BRAF V600E mutation. N Engl J Med. 2011;364(26):2507-16.

19. O'Brien SG, Guilhot F, Larson RA, et al. Imatinib compared with interferon and lowdose cytarabine for newly diagnosed chronicphase chronic myeloid leukemia. N Engl J Med. 2003;348(11):994-1004.

20. Demetri GD, von Mehren $M$, Blanke CD, et al. Efficacy and safety of imatinib mesylate in advanced gastrointestinal stromal tumors. N Engl J Med. 2002;347(7):472-80.

21. Verheijen RB, $\mathrm{Yu} \mathrm{H}$, Schellens JHM, et al. Practical Recommendations for Therapeutic Drug Monitoring of Kinase Inhibitors in Oncology. Clin Pharmacol Ther. 2017;102 (5):765-76.

22. de Wit D, Guchelaar HJ, den Hartigh J, et al. Individualized dosing of tyrosine kinase inhibitors: are we there yet? Drug Discov Today. 2015;20(1):18-36.

23. Yu H, Steeghs N, Nijenhuis CM, et al. Practical guidelines for therapeutic drug monitoring of anticancer tyrosine kinase inhibitors: focus on the pharmacokinetic targets. Clin Pharmacokinet. 2014;53(4):305-25.

24. de Jonge ME, Huitema AD, Schellens JH, et al. Individualised cancer chemotherapy: 
strategies and performance of prospective studies on therapeutic drug monitoring with dose adaptation: a review. Clin Pharmacokinet. 2005;44(2):147-73.

25. Bardin C, Veal G, Paci A, et al. Therapeutic drug monitoring in cancer--are we missing a trick? Eur J Cancer. 2014;50(12):2005-9.

26. van der Heijden J, de Beer $Y$, Hoogtanders $K$, et al. Therapeutic drug monitoring of everolimus using the dried blood spot method in combination with liquid chromatographymass spectrometry. J Pharm Biomed Anal. 2009;50(4):664-70.

27. Andersen TF, Madsen $M$, Jørgensen J, et al. The Danish National Hospital Register. A valuable source of data for modern health sciences. Dan Med Bull. 1999;46(3):263-8.

28. Herrett E, Gallagher AM, Bhaskaran K, et al. Data Resource Profile: Clinical Practice
Research Datalink (CPRD). Int J Epidemiol 2015;44(3):827-36.

29. Smelick GS, Heffron TP, Chu L, et al. Prevalence of acid-reducing agents (ARA) in cancer populations and ARA drug-drug interaction potential for molecular targeted agents in clinical development. Mol Pharm. 2013;10(11):4055-62.

30. Willemsen AECAB, Lubberman FJ, Tol J, et al. Effect of food and acid-reducing agents on the absorption of oral targeted therapies in solid tumors. Drug Discov Today. 2016;21(6):96276.

31. Shipkova M, Hesselink DA, Holt DW, et al. Therapeutic Drug Monitoring of Everolimus: A Consensus Report. Ther Drug Monit. 2016;38(2):143-69. 
0

$\varnothing$

$Q$

$\curvearrowright$

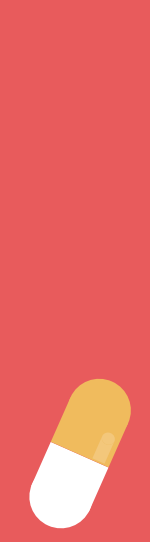

0
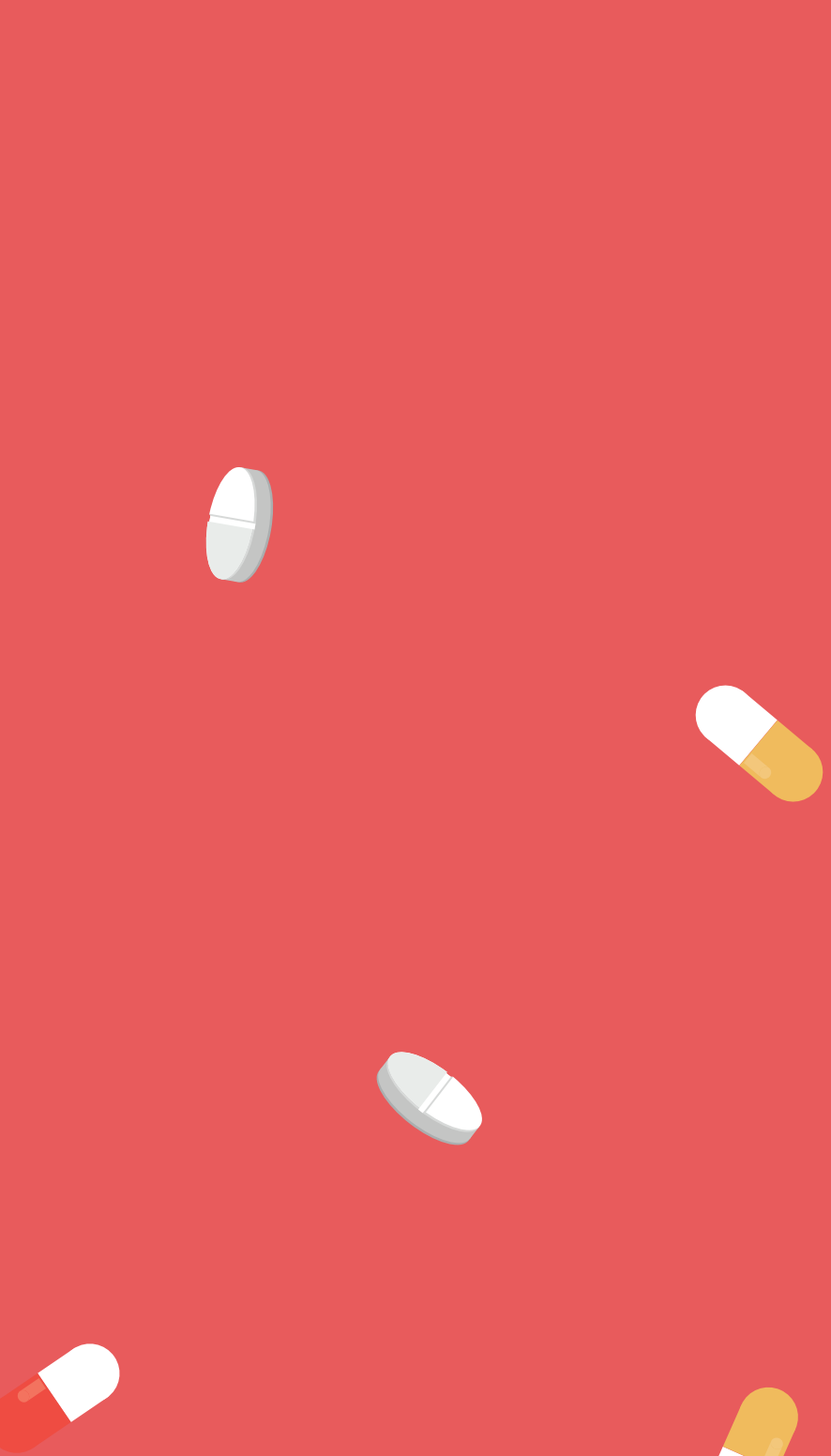
Chapter

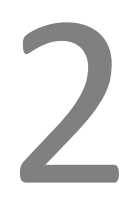

The impact of medications on cancer risk in real-life populations 



\section{Chapter}

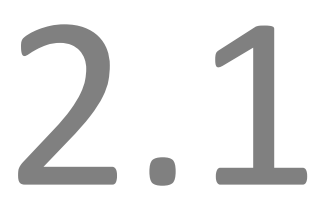

\section{Use of biguanides and the risk of colorectal}

cancer: a register-based cohort study

L.M. Knapen, S.T.A.M. Dittrich, F. de Vries, J. Starup-Linde, P. Vestergaard, R.M.A. Henry, L.M.L. Stolk, C. Neef, M.T. Bazelier

Curr Drug Saf. 2013;8(5):349-56 


\section{Abstract}

\section{Background}

Observational studies have shown conflicting results on the potential protecting effect of biguanide use with the risk of colorectal neoplasms. In addition, the cellular mechanism can either support or oppose biguanides influence on colorectal carcinoma.

\section{Objectives}

Our objective was to evaluate the association between biguanide use and colorectal carcinoma.

\section{Patients and methods}

A population-based cohort study using healthcare data from the Danish National database (1996-2007), was conducted. Oral antidiabetic (OAD) drug users $(n=177,281)$ were matched 1:3 with a population-based reference group. Cox proportional hazard models estimated hazard ratios (HRs) of colorectal carcinoma. Stratification was performed to analyse the risk of colorectal cancer in current biguanide users. Two sub-analyses were performed, to investigate the risk of colorectal cancer associated with discontinuous and prolonged use of biguanides.

\section{Results}

Instead of a protective effect, we found that current biguanide users had a 1.2-fold increased risk of colorectal cancer (HR 1.19; 95\% confidence interval (CI) 1.08-1.30) as compared with the non-diabetes reference group. Prolonged use was not inversely associated with colorectal cancer either. When studying colorectal risk with biguanides, the underlying type 2 diabetes mellitus (T2DM) should be taken into account since a 1.3-1.6-fold increased risk was found in OAD drug users compared to controls unexposed to diabetic medication.

\section{Conclusions}

This study could not detect a protective effect of biguanide use with colorectal cancer. Therefore, this study does not support a further investigation of the effectiveness of biguanides to prevent colorectal carcinoma in clinical studies. 


\section{Introduction}

Type 2 diabetes mellitus (T2DM) is a major threat to human health in the 21st century ${ }^{1}$. The prevalence of diabetes mellitus is expected to rise over the next decades, from an estimated 171 million patients in 2000 to 366 million in 2030 worldwide ${ }^{2}$. T2DM is characterised by insulin resistance and relative insulin deficiency and can lead to severe macro- and microvascular complications, such as coronary heart disease, nephropathy, retinopathy and neuropathy ${ }^{1}$. The biguanide metformin is the most frequently used drug in the management of $\mathrm{T}^{2} \mathrm{DM}^{3}$. Metformin is known to improve insulin resistanceassociated hyperinsulinemia and hyperglycaemia mainly by affecting the insulin/IGF-1 signalling pathway ${ }^{3,4}$. Since insulin resistence is a risk factor of cancer, metformin may have protective effects on various types of malignancies ${ }^{5,6}$. Metformin indirectly activates AMP-activated protein kinase (AMPK)-signalling ${ }^{4}$. Activated AMPK improves insulin-sensitivity, and lowers circulating insulin, which leads to suppression of the stimulatory effects of obesity and hyperinsulinemia on tumour growth. This association has been studied for lung, breast, prostate and colorectal cancer ${ }^{5}$. Activation of AMPK also inhibits the mammalian target of rapamycin-signalling pathway (mTOR), which is one of the most frequently deregulated molecular networks in human cancer ${ }^{4}$. Inhibition of this pathway leads to reduced cell growth and proliferation, protein synthesis, cell metabolism and tumour angiogenesis ${ }^{5,6}$. Meta-analyses suggested a 0.68 -fold and 0.64 -fold decreased risk of colorectal cancer in metformin users ${ }^{4,7}$. Furthermore, a pooled analysis of five epidemiological studies suggested a $37 \%$ reduced risk of colorectal cancer in metformin users ${ }^{8}$. In contrast, T2DM itself has been associated with a $27-30 \%$ increased risk of colorectal cancer in several meta-analyses ${ }^{9-12}$.

However, various observational studies have shown conflicting results. Two independent groups of researchers analysed the world's largest primary care database, the United Kingdom (UK) Clinical Practice Research Datalink (CPRD). They did not report protective effects of metformin on the risk of colorectal cancer $^{13}$, or even a 1.4-fold increased risk $^{14}$. In contrast, a $40 \%$ reduced risk of colorectal cancer with new users of metformin monotherapy (as compared to prevalent users of insulins) was reported when a different UK General Practice Database ('The Health Improvement Network') was analysed ${ }^{15}$. Alternative methodological explanations for these discrepancies include the presence of selection bias, different definitions of exposure windows (metformin may need several years to exert protective effects on malignancies), or different (clinical) characteristics of exposed and control patients whose hazard may have varied over time. This has been described previously when the association between hormone replacement therapy (HRT) and cardiovascular disease was evaluated, and is sometimes referred to as the 'healthy HRT user effect'. A 'new-user study design' may deal with this distortion by restricting the study to patients who have recently started taking metformin and comparing those to recent starters of other 
antidiabetic drugs ${ }^{16}$. Therefore, objective of our study was to determine the risk of colorectal cancer in biguanide users.

\section{Research design and methods}

\section{Data source}

This study used healthcare data from the widely used Danish National databases. In Denmark, separate registers with computerised medical records on all contacts to hospitals, and on the use of drugs can be linked to the entire population (approximately 5.5 million inhabitants) ${ }^{17}$. The Ministry of Interior keeps records of all inhabitants and their migrations, date of birth and (cause of) death. Information on hospital admissions comes from the National Hospital Discharge Register ${ }^{17}$, which covers all inpatient contacts from 1977 to 1994 and since 1995 also all outpatient visits to hospitals, outpatient clinics, and emergency rooms. Upon discharge, the physician codes the reason for the contact according to the International Classification of Diseases (ICD) system. The data quality of the register is high, with an estimated completeness of $93.4 \%$ and a positive prediction value of $88.9 \%$ for colorectal carcinoma ${ }^{18}$. The Danish Medicines Agency keeps a nationwide register of all prescription drugs sold at pharmacies throughout the country from 1996 onward (National Pharmacological Database run by the Danish Medicines Agency, http://www.dkma.dk). All dispensings are registered with ATC code, dosage, and date. As all dispensings are registered to the individual who redeems the prescription, the capture and validity are high ${ }^{18}$. All registers can be linked through the use of a person specific code (the civil person number) given to all inhabitants, and can be used for all of the registrations mentioned before.

\section{Study population}

The exposed study population consisted of all patients (aged 18+) with at least two prescriptions of antidiabetic medication (1996-2007). Patients with a history of colorectal cancer or a history of bowel resection were excluded from the analysis. The date of the first antidiabetic prescription within the period of valid data collection defined the index date. Patients with only a recorded prescription of insulin on their index date (i.e. no oral antidiabetic (OAD) prescription) were excluded from the cohort. Therefore, all patients had a record of an OAD drug prescription on their index date. OAD drug users were matched to up to three control persons, who did not have an antidiabetic ( $O A D$ or insulin) prescription any time during follow-up. Controls were assigned the same index date as their matched OAD drug user. All participants were then followed from their index date to the end of data collection, emigration or the patient's death, whichever came first. 


\section{Exposure}

The total period of follow-up for each patient was divided into periods of current and past exposure, with patients moving between these groups. Each period started on the day of a new antidiabetic prescription and ended 3 months after the expected duration of antidiabetic therapy, or on the date that a new antidiabetic drug was prescribed within this period. For OAD therapy, the expected duration of use was defined as the median time between two OAD dispensings, based on all OAD dispensings. For insulin treatment, the median time between two insulin dispensings (based on all insulin dispensings) was taken as the expected duration of use.

At the start of each interval, each patient was classified as a current user of antidiabetic medication if they had an antidiabetic prescription on that start date or in the three months before. The current user status was determined for five different groups of antidiabetic medication: biguanides, sulphonylureas, thiazolidinediones (TZDs), insulin, or other antidiabetics (including glinides, alpha glucosidase inhibitors, dipeptidyl peptidase-4 inhibitors (DPP4-Is) and glucagon-like peptide-1 (GLP-1) receptor agonists). For biguanides, recent and past users were also identified. Patients were classified as recent users if they had a biguanide prescription between three and twelve months before the start date of an interval. A biguanide prescription more than twelve months before the start date was defined as past use. For the reference group, the total period of follow-up was divided into periods of six months. Current exposure to biguanides was stratified to the number of dispensings ever before.

Denmark is one of the few countries where the biguanide phenformin is still registered. Since there is pre-clinical evidence that both metformin and phenformin have an effect on the AMPK-signalling pathway ${ }^{19}$, biguanides were analysed as a group.

\section{Study Outcome}

Patients were followed up for the occurrence of a first event of colorectal cancer (ICD-10 codes: C18-C21 and D010-D013) as recorded in the National Hospital Discharge Register.

\section{Risk factors}

The presence of risk factors was assessed by reviewing the computerised medical records for any evidence of these risk factors before the start of each interval. A potential confounder that was determined at baseline is sex. For a time-dependent analysis, the following potential confounders were considered: age, a history of malignancies other than colorectal cancer, a family history of hereditary colon cancer syndromes, physical activity, inflammatory bowel disease, ischemic heart disease, dietary fiber, fruit, vegetables, as well as a prescription for anti-neoplastic drugs ever 
before or a prescription for statins ${ }^{20}$, aspirin, non steroidal anti-inflammatory drugs (NSAIDs), bisphosphonates, and calcium supplements within the previous 6 months.

\section{Statistical analysis}

Cox proportional hazards analysis was used. First, we compared the risk of colorectal cancer in never, current, recent and past users of biguanides with that in control patients (without diabetes). Second, we compared the risk of colorectal cancer in current, recent and past users of biguanides with that in never users of biguanides (other OAD drug users). In these analyses, the calculations were adjusted for all potential confounders that changed the beta coefficient of the hazard ratio $(H R)>5 \%$ in an age/sex adjusted analysis or potential confounders that were clinically relevant ${ }^{21-25}$. To investigate a duration of use relationship, we stratified the risk of colorectal cancer in current biguanide users by the number of biguanide dispensings ever before $(\leq 10$ dispensings (short term), 11-30 dispensings (intermediate term), >30 dispensings (long term)). Wald tests were used to examine if there were statistically significant differences between differently exposed groups. All data management and statistical analyses were conducted using SAS 9.1/9.2 software.

\section{Sensitivity analysis}

In a sensitivity analysis, we studied the impact of a 'new user design'. The full cohort of OAD drug users was restricted to those patients with a first ever prescription of antidiabetic medication with a lead-in period of 1 year of valid time.

\section{Results}

We included 177,281 OAD drug users, who were matched with 477,647 populationbased controls. For the exposed patients, $52.9 \%$ of all patients were male, and mean age at index date was 62.4 years. OAD drug users were more likely to have a history of ischemic heart disease than the reference group (16.4\% versus $8.2 \%$ ). Also, the occurrence of malignancies other than colorectal cancer ever before was slightly higher in OAD drug users than in the reference group (7.2\% versus $6.2 \%$ ). OAD drug users used more NSAIDs, statins and aspirin. During follow-up, $70.1 \%$ of the OAD drug users received a prescription for a biguanide at least once, of which $0.5 \%$ was a prescription for phenformin. Sulphonylureas were used by $76.6 \%$, thiazolidinediones by $4.5 \%$, insulins by $25.8 \%$ and other antidiabetic drugs by $10.5 \%$ of the OAD drug users. Further baseline characteristics are shown in Table 2.1.1.

Table 2.1.2 shows that biguanide users had a 1.2-fold increased risk of colorectal cancer (HR 1.19; 95\% Cl 1.08-1.30) as compared with the non-diabetes reference group. 
T2DM itself was associated with colorectal cancer, with risk of colorectal cancer ranging from 1.3-1.6.

Table 2.1.1 Baseline characteristics of oral antidiabetic drug users and the reference group ${ }^{\mathrm{a}}$.

\begin{tabular}{lcc}
\hline & $\begin{array}{c}\text { Oral antidiabetic drug } \\
\text { users } \\
\text { Characteristic }\end{array}$ & Reference group \\
\hline Mean duration of follow-up after index date (years) & 5.3 & $\mathrm{~N}=477,647$ \\
Sex & & 6.2 \\
Females & $83,425(47.1)$ & $226,459(47.4)$ \\
Males & $93,856(52.9)$ & $251,188(52.6)$ \\
Age & & \\
Mean at index date (years) & 62.4 & 62.3 \\
Median at index date (years) & 63 & 63 \\
By category & & $81,478(17.1)$ \\
18 - 49 years & $31,027(17.5)$ & $109,980(23.0)$ \\
50 - 59 years & $39,707(22.4)$ & $130,177(27.3)$ \\
60 - 69 years & $46,711(26.3)$ & $106,576(22.3)$ \\
$70-79$ years & $39,548(22.3)$ & $49,436(10.3)$ \\
$80+$ years & $20,288(11.4)$ & \\
Comorbidity presence ever before & & $29,852(6.2)$ \\
Malignancy other than colorectal cancer & $12,721(7.2)$ & $1,737(0.4)$ \\
Hereditary colon cancer syndromes & $794(0.4)$ & $3,269(0.7)$ \\
Inflammatory bowel disease & $1,576(0.9)$ & $39,119(8.2)$ \\
Ischemic heart disease & $29,115(16.4)$ & \\
Drug use within 6 months before & & $56,704(11.9)$ \\
NSAIDs & $28,920(16.3)$ & $20,910(4.4)$ \\
Statins & $21,269(12.0)$ & $41,391(8.7)$ \\
Aspirin & $29,267(16.5)$ & $4,376(0.9)$ \\
Bisphosphonates & $1,123(0.6)$ & $2,963(0.6)$ \\
Calcium supplements & $988(0.6)$ &
\end{tabular}

NSAIDs: non steroid anti-inflammatory drugs. ${ }^{a}$ Data are $n,(\%)$ unless stated otherwise.

Table 2.1.3 shows that there were no statistically significant differences of developing colorectal cancer in biguanide users compared with users of other antidiabetic drugs (HR 0.95; 95\% $\mathrm{Cl}$ 0.87-1.04), who never used a biguanide. Two analyses were performed to study the influence of time. The duration of use was studied by the number of prescriptions. No association was found with prolonged use of biguanides (HRs 0.94-0.97). Time since discontinuation was determined by studying recent and past users of biguanides. There were no statistically significant differences between current biguanide use versus past use. However, patients who recently stopped using biguanides seemed to have a slightly increased risk on developing colorectal cancer (HR 1.25; 95\% Cl 1.05-1.50), which was statistically significant with current use and with past use. 


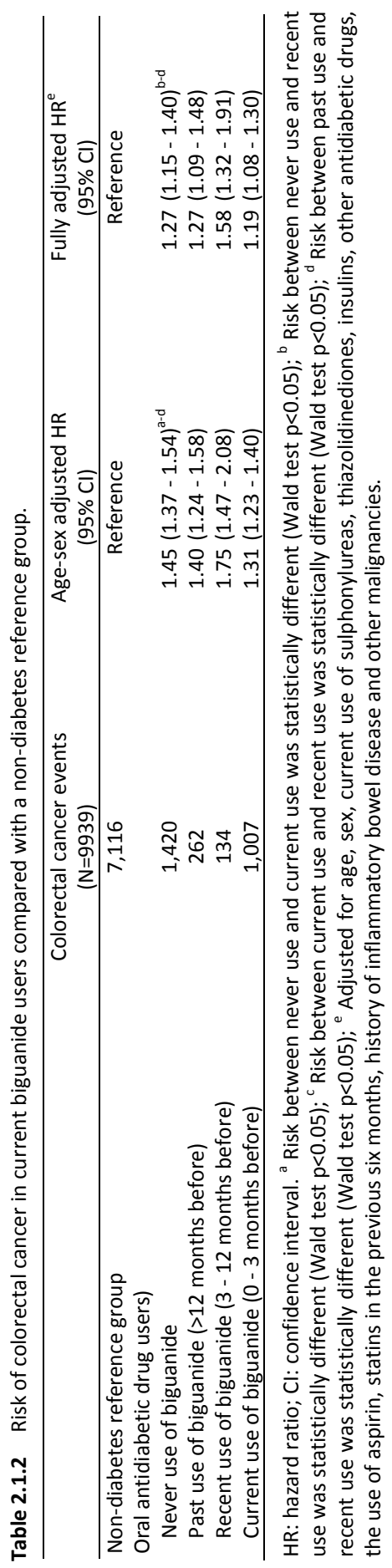




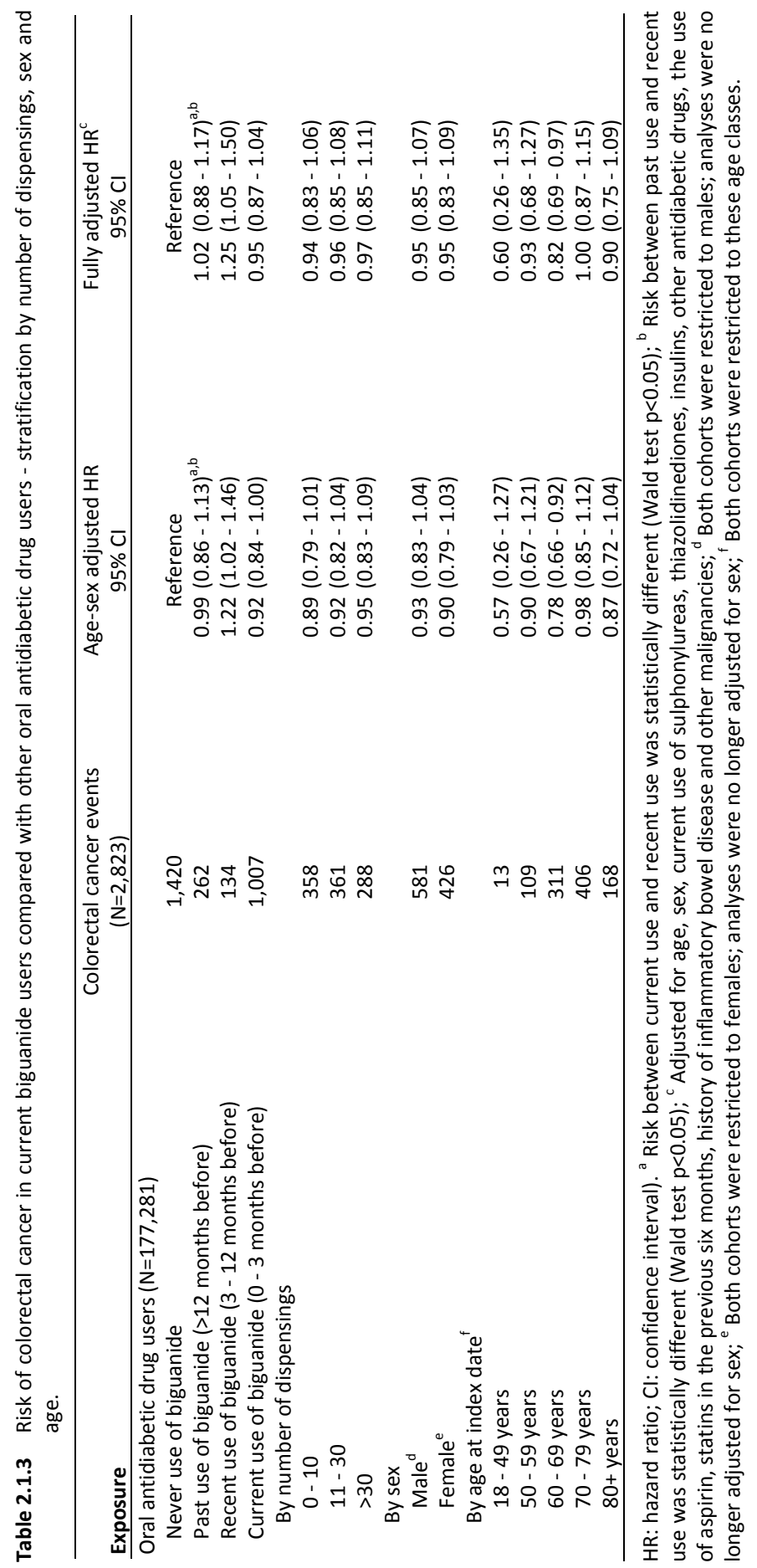


We found that biguanide users with a history of malignancy other than colorectal cancer have an 1.2-fold increased risk on developing colorectal cancer than patients without that history (HR 1.23; 95\% Cl 1.02-1.49). The same association was found for biguanide users with a history of hereditary colon cancer syndromes (HR 2.85; $95 \% \mathrm{Cl} 1.91-4.25)$ and a history of inflammatory bowel disease (HR 1.76; $95 \% \mathrm{Cl}$ 1.06-2.94), as shown in Table 2.1.4.

Table 2.1.4 Risk of colorectal cancer in current biguanide users compared with other oral antidiabetic drug users by potential determinants of colorectal cancer.

\begin{tabular}{|c|c|c|}
\hline Exposure & $\begin{array}{l}\text { Number of events of } \\
\text { Colorectal cancer } \\
(\mathrm{N}=2,823)\end{array}$ & Fully adj $\mathrm{HR}^{\mathrm{a}}(95 \% \mathrm{Cl})$ \\
\hline \multicolumn{3}{|l|}{ Oral antidiabetic drug users $(\mathrm{N}=177,281)$} \\
\hline Never use of biguanide & 1,420 & Reference \\
\hline Past use of biguanide (>12 mnd before) & 262 & $1.02(0.88-1.17)^{\mathrm{b}, \mathrm{c}}$ \\
\hline Recent use of biguanide ( 3 - 12 mnd before) & 134 & $1.25(1.05-1.50)$ \\
\hline Current use of biguanide ( 0 - 3 mnd before) & 1,007 & $0.95(0.87-1.04)$ \\
\hline \multicolumn{3}{|l|}{ By a history of comorbidities } \\
\hline \multicolumn{3}{|l|}{ Malignancy other than colorectal cancer } \\
\hline Yes & 122 & $1.23(1.02-1.49)^{d}$ \\
\hline No & 885 & $0.92(0.84-1.01)$ \\
\hline \multicolumn{3}{|l|}{ Hereditary colon cancer syndromes } \\
\hline Yes & 25 & $2.85(1.91-4.25)^{d}$ \\
\hline No & 982 & $0.94(0.86-1.03)$ \\
\hline \multicolumn{3}{|l|}{ Inflammatory bowel disease } \\
\hline Yes & 15 & $1.76(1.06-2.94)^{d}$ \\
\hline No & 992 & $0.95(0.87-1.04)$ \\
\hline \multicolumn{3}{|l|}{ Ischemic heart disease } \\
\hline Yes & 244 & $1.06(0.92-1.22)$ \\
\hline No & 763 & $0.93(0.84-1.02)$ \\
\hline \multicolumn{3}{|l|}{ By drug exposure 6 months before } \\
\hline \multicolumn{3}{|l|}{ NSAIDs } \\
\hline Yes & 192 & $0.89(0.76-1.04)$ \\
\hline No & 815 & $0.97(0.88-1.07)$ \\
\hline \multicolumn{3}{|l|}{ Statins } \\
\hline Yes & 356 & $0.91(0.80-1.04)$ \\
\hline No & 651 & $0.96(0.88-1.06)$ \\
\hline \multicolumn{3}{|l|}{ Aspirin } \\
\hline Yes & 399 & 0.97 (0.86 - 1.09) \\
\hline No & 608 & $0.94(0.85-1.04)$ \\
\hline \multicolumn{3}{|l|}{ Bisphosphonates } \\
\hline Yes & 20 & $1.87(1.20-2.92)$ \\
\hline No & 987 & $0.95(0.87-1.03)$ \\
\hline \multicolumn{3}{|l|}{ Calcium supplements } \\
\hline Yes & 15 & 2.02 (1.21 - 3.37) \\
\hline No & 992 & $0.95(0.87-1.03)$ \\
\hline
\end{tabular}

adj: adjusted; $\mathrm{Cl}$ : confidence interval; HR: hazard ratio; NSAIDs: non steroidal anti-inflammatory drugs. ${ }^{a}$ Adjusted for age, sex, current use of sulphonylureas, thiazolidinediones, insulins, other antidiabetic drugs, the use of aspirin, statins in the previous six months, history of inflammtory bowel disease and other malignancies. ${ }^{b}$ Risk between past use and recent use was statistically different (Wald test $p<0.05$ ); ${ }^{c}$ Risk between current use and recent use was statistically different (Wald test $p<0.05$ ); ${ }^{d}$ Risk between patients with a history of comorbidity versus patients without a history of comorbidity was statistically different (Wald test $p<0.05) ;{ }^{e}$ Risk of drug use 6 months before versus no drug use 6 months before was statistically different (Wald test $\mathrm{p}<0.05$ ). 
All these hazard ratios were statistically significant. Furthermore, previous 6 months drug use was associated with an increased risk with bisphosphonate (HR 2.02) and calcium supplement (HR 1.87) use, while no altered risk was found with use of NSAIDs, statins or aspirin.

In a sensitivity analysis, we used a 'new user design' and restricted our cohort to starters of diabetes treatment. This may have removed potential differential hazards over time from prevalent users. No differences were found when compared with our main analysis in Table 2.1.3. Again, there were no significant differences on developing colorectal cancer in new biguanide users compared with new users of other antidiabetic drugs (HR 1.06; 95\% Cl 0.93-1.20). There were no significant differences between current biguanide users versus past users (HR 0.91; $95 \% \mathrm{Cl} 0.72-1.15$ ). The 'new user design' also showed that patients who recently stopped using biguanides seemed to have a slightly increased risk on developing colorectal cancer (HR 1.47; $95 \% \mathrm{Cl} 1.15-1.86)$, which was statistically significant with current use and with past use.

\section{Discussion}

The main finding of our study is that current biguanide users had a 1.2-fold increased risk of developing colorectal cancer as compared to a non-diabetes reference group. However, this increased risk was mainly explained by the underlying disease (T2DM) because there was no statistically significant difference in the risk between current biguanide users and users of other antidiabetic drugs. The duration of use did not further increase or decrease the risk. Restricting our analysis to incident biguanide users did not change our findings.

Our main findings are in line with data from two randomised controlled trials and several observational studies. The ADOPT (A Diabetes Outcome Progression Trial) and the RECORD (Rosiglitazone Evaluated for Cardiovascular Outcomes and Regulation of glycaemia in Diabetes) trials by Home et al. did not find a protective effect of metformin (compared to rosiglitazone) on developing several subtypes of cancer, including colorectal cancer ${ }^{26}$. A recent meta-analysis of randomised controlled trials further supports that metformin does not have a protective effect on developing cancer in general ${ }^{27}$. In addition, two observational studies by Bodmer et al. and Yang et al. studying the UK Clinical Practice Research Datalink (CPRD) found a tendency towards an increased risk with prolonged use of metformin and overall no association with colorectal carcinoma ${ }^{13,14}$.

Previous studies that were not in line with our study included observational studies which demonstrated a protective effect of metformin on colorectal cancer. One example is the retrospective cohort study by Lee et al. $^{28}$. However, the Charlson comorbidity index was used in this study for statistical adjustments, which has probably 
resulted in substantial residual confounding. Currie et al. conducted a retrospective cohort study in The Health Information Network (THIN) and found a 40\% reduced risk of colorectal cancer with new users of metformin monotherapy (as compared to prevalent users of insulins) $)^{15}$. An important fact is that of all general practices which contributed data to the THIN database, 327 (66\%) also provided their data to the CPRD database in $2012^{29}$. The CPRD database was also used by Bodmer et al. and Yang et al. ${ }^{13,14}$. The conflicting results between these studies can probably be explained by selection bias, different definitions of exposure windows (metformin may need several years to exert protective effects on malignancies), or the use of different codelists for clinical outcomes ${ }^{30,31}$. UK GP databases are very large with hundreds of millions of medical and prescription records; advanced computer programming skills are required and a programming error cannot be excluded. These explanations can only be evaluated by the researchers of the original publications. Another explanation is that the clinical characteristics of exposed and control patients varied among these studies; in addition, their hazard may have also varied over time or different types of statistical adjustment. It has been proposed by Ray et al. that a sensitivity analysis by using a 'new user design' could deal with this ${ }^{16}$, in particular because a $40 \%$ reduction of (gastrointestinal) malignancies with metformin use has been reported in an observational study with incident users ${ }^{32}$. After we restricted our cohort to 'incident' or 'new users', our main results did not change and therefore this bias is unlikely to have played a role in the current study.

T2DM itself could also influence the risk of colorectal cancer, which is in line with findings of this study. A meta-analysis of six case-control studies and nine cohort studies showed that diabetes mellitus was associated with a 1.3-fold increased risk of colorectal cancer (95\% Cl 1.20-1.40) compared to non-diabetes controls. Besides that, diabetes was positively associated with colorectal cancer mortality ${ }^{33}$. In 2010, a consensus report was published which stated that diabetes is associated with an increased risk of several cancers, including colon and rectum cancer ${ }^{34}$. Several observational studies also found an effect of insulin on the risk of colorectal cancer. Yang et al. found that chronic insulin therapy significantly increased the risk of colorectal cancer in patients with T2DM (OR 1.21; 95\% Cl 1.03-1.42) ${ }^{13}$. The hazard ratios in this study are adjusted for the use of other antidiabetic drugs, including insulin. It could be argued that a protective effect of biguanides is concealed by a non-protecting effect of T2DM itself. To eliminate this effect, we compared OAD drug users with a nondiabetes reference group. In addition, we were not able to detect protective effects of biguanides when we compared exposure to other antidiabetic drug users, who could possibly have been exposed to insulins.

Several mechanisms have been studied to explore the association between diabetes and cancer, such as hyperinsulinaemia, hyperglycaemia and chronic inflammation ${ }^{34,35}$. The insulin receptor, which is expressed on most cancer cells, is capable of inducing mitogenesis, proliferation and metastasis ${ }^{34,35}$. It also activates multiple signalling 
pathways, which may stimulate proliferation, protection from apoptotic stimuli, invasion and metastasis. Hyperinsulinaemia could promote carcinogenesis by increasing insulin-like growth factor 1 (IGF-1), which acts as a growth stimulus on cancer cells ${ }^{34}$. Hyperglycaemia is also associated with increased neoplastic growth, which is supported by the dependence of many cancers on glycolysis for energy and their highly effective glucose uptake mechanisms, but is contradicted by some in vivo models ${ }^{34,35}$. In addition to the direct effects of insulin and glucose, there is evidence that T2DM itself might enhance other pathways resulting in malignant progression, for example by releasing the cytokine interleukine- $6^{34}$.

Strengths of our study included a reasonable sample size and a mean duration of follow-up of at least 5 years, which allowed us to evaluate the association between biguanide use and the risk of colorectal cancer. Our source population included the total Danish population and the reference group was therefore population-based. There was detailed longitudinal information on drug prescribing. Our study had several limitations. Data on risk factors of colorectal cancer such as obesity, smoking, physical activity, a family history of colorectal cancer, fruit, vegetables, dietary fiber and the consumption of red meat were not available and may have biassed our findings. Obesity may have masked the potential protective effect of metformin use, since obesity is associated with an increased risk for developing colorectal cancer and metformin users generally have a higher body mass index (BMI) as compared to OAD drug users who use sulphonylureas ${ }^{36-38}$. The same applies for smoking, which has been associated with a 2-fold risk of adenomatous polyps. The prevalence of cigarette smoking is higher in diabetes patients as compared to controls ${ }^{39}$. Furthermore, physical activity may have masked the potential protective effect of metformin use, since excess weight, due to less physical activity, is the most important risk factor for the development of type two diabetes ${ }^{40}$. Decreased physical activity appears to increase the risk for cancer ${ }^{41}$. However, after adjustment for BMI and smoking, two observational studies were still unable to detect protective effects of metformin with colorectal cancer ${ }^{13,14}$. To our best knowledge, differential effects of red meat intake in diabetes patients compared to non-diabetes controls has not been reported ${ }^{38}$, and it is therefore unlikely to have biassed the association with colorectal cancer.

In conclusion, our study does not show any protective effects of biguanide use with the risk of colorectal carcinoma. An indirect test of this hypothesis which supported our main finding was the absence of an effect with the duration of use. Therefore, this study does not support a further investigation of the effectiveness of biguanides to prevent colorectal carcinoma in clinical studies. 


\section{References}

1. Israili $\mathrm{ZH}$. Advances in the treatment of type 2 diabetes mellitus. Am J Ther. 2011;18(2): 117-52.

2. Wild S, Goglic G, Green A, et al. Global Prevalence of Diabetes. Estimates for the year 2000 and projections for 2030. Diabetes Care. 2004;27(5):1047-53.

3. Wulffelé MG, Kooy A, Zeeuw D de, et al. The effect of metformin on blood pressure, plasma cholesterol and triglycerides in type 2 diabetes: a systematic review. J Intern Med. 2004;256;1-14.

4. Noto H, Goto A, Tsujimoto T, et al. Cancer risk in diabetic patients treated with metformin: a systematic review and meta-analysis. PLoS One. 2012;7(3):e33411.

5. Dowling RJO, Goodwin PJ, Stambolic V. Understanding the benefit of metformin use in cancer treatment. BMC Med. 2011;9:33.

6. Shaw RJ, Lamia KA, Vasquez $D$, et al. The kinase LKB1 mediates glucose homeostasis in liver and therapeutic effects of metformin. Science. 2005;310(5754):1642-6.

7. Soranna D, Scotti L, Zambon A, et al. Cancer risk associated with use of metformin and sulfonylurea in type 2 diabes: a meta-analysis. Oncologist. 2012;17(6):813-22.

8. Zhang ZJ, Zheng ZJ, Kan H, et al. Reduced risk of colorectal cancer with metformin therapy in patients with type 2 diabetes. Diabetes Care. 2011;34;2323-8.

9. Jiang $Y$, Ben $Q$, Shen $H$, et al. Diabetes mellitus and incidence and mortality of colorectal cancer: a systematic review and meta-analysis of cohort studies. Eur J Epidemiol. 2011;26(11):863-76.

10. Krämer HU, Schöttker B, Raum E, et al. Type 2 diabetes mellitus and colorectal cancer: metaanalysis on sex-specific differences. Eur J Cancer. 2012;48(9):1269-82.

11. Larsson SC, Orsini N, Wolk A. Diabetes mellitus and risk of colorectal cancer: a metaanalysis. J Natl Cancer Inst. 2005;97(22):167987.

12. Luo W, Cao Y, Liao C, et al. Diabetes mellitus and the incidence and mortality of colorectal cancer: A meta-analysis of twenty four cohort studies. Colorectal Disease. 2011;14(11): 1307-12.

13. Yang YX, Hennesy S, Lewis JD. Insulin therapy and colorectal cancer risk among type 2 diabetes mellitus patients. Gastroenterology. 2004;127;1044-50.

14. Bodmer M, Becker C, Meier C, et al. Use of metformin is not associated with a decreased risk of colorectal cancer: a case-control analysis. Cancer Epidemiol Biomarkers Prev. 2012;21(2):280-6.

15. Currie CJ, Poole CD, Gale EAM. The influence of glucose-lowering therapies on cancer risk in type 2 diabetes. Diabetologia. 2009;52(9): 1766-77.

16. Ray WA. Evaluating medication effects outside of clinical trials: new-user designs. Am J Epidemiol. 2003;158(9):915-20.

17. Andersen TF, Madsen $M$, Jørgensen J, et al. The Danish National Hospital Register. A valuable source of data for modern health sciences. Dan Med Bull. 1999;46(3):263-8.

18. Helqvist L, Erichsen R, Gammelager $\mathrm{H}$, et al. Quality of ICD-10 colorectal cancer diagnosis codes in the Danish National Registry of Patients. Eur J Cancer Care. 2012;21(6):722-7.

19. Hardie DG. AMP-activated protein kinase as a drug target. Annu Rev Pharmacol Toxicol. 2007;47:185-210.

20. Poynter JN, Gruber SB, Higgins PD, et al. Statins and the risk of colorectal cancer. N Engl J Med. 2005; 352(21):2184-92

21. Bardou M, Barkun A, Martel M. Effect of statin therapy on colorectal cancer. Gut. 2010; 59:1572-85.

22. Chan AT, Giovannucci EL, Meyerhardt JA, et al. Long-term use of aspirin and nonsteroidal anti-inflammatory drugs and risk of colorectal cancer. JAMA. 2005;294:914-23.

23. Ekbom A, Helmick $C$, Zack $M$, et al. Ulcerative colitis and colorectal cancer. A populationbased study. N Engl J Med. 1990; 323(18):1228-33.

24. Jasperson KW, Tuohy TM, Neklason DW, et al. Hereditary and familial colon cancer. Gastroenterology. 2010;138(6):2044-58.

25. Rothwell PM, Price JF, Fowkes GR, et al. Shortterm effects of daily aspirin on cancer incidence, mortality, and non-vascular death: analysis of the time course of risks and benefits in 51 randomised controlled trials. Lancet. 2012;379:1602-12.

26. Home PD, Kahn SE, Jones NP, et al; ADOPT Study Group; RECORD Steering Committee. Experience of malignancies with oral glucose- 
lowering drugs in the randomised controlled ADOPT (A Diabetes Outcome Progression Trial) and RECORD (Rosiglitazone Evaluated for Cardiovascular Outcomes and Regulation of Glycaemia in Diabetes) clinical trials. Diabetologia. 2010;53(9):1838-45.

27. Stevens RJ, Ali R, Bankhead CR, et al. Cancer outcomes and all-cause mortality in adults allocated to metformin: systematic review and collaborative meta-analysis of randomised clinical trials. Diabetologia. 2012;55:2593-603.

28. Lee MS, Hsu CC, Wahlqvist ML, et al. Type 2 diabetes increases and metformin reduces total, colorectal, liver and pancreatic cancer incidences in Taiwanese: a representative population prospective cohort study of 800,000 individuals. BMC Cancer. 2011;11:20.

29. Cai B, Xu W, Bortnichak E, et al. An algorithm to identify medical practices common to both the General Practice Research Database and The Health Improvement Network database. Pharmacoepidemiol Drug Saf. 2012;21(7): 770-4.

30. De Vries F, de Vries C, Cooper C, et al. Reanalysis of two studies with contrasting results on the association between statin use and fracture risk: the general practice research database. Int J Epidemiol. 2006;35(5):1301-8.

31. De Vries F, van Staa TP, Leufkens HG. Proton pump inhibitors, fracture risk and selection bias: three studies, same database, two answers. Osteoporos Int. 2011;22(5):1641-2.

32. Libby G, Donnelly LA, Donnan PT, et al. New users of metformin are at low risk of incident cancer: a cohort study among people with type 2 diabetes. Diabetes Care. 2009;32(9):1620-5.
33. Larsson SC, Orsini N, Wolk A. Diabetes mellitus and risk of colorectal cancer: a metaanalysis. J Natl Cancer Inst. 2005;97(22): 1679-87.

34. Giovannucci E, Harlan D, Archer MC, et al. Diabetes and cancer: a consensus report. Diabetes Care. 2010;33:1674-85.

35. Vigneri P, Frasca F, Sciacca L, et al. Diabetes and cancer. Endocr Relat Cancer. 2009;16:1103-23.

36. Roumie $\mathrm{CL}$, Liu $\mathrm{X}$, Choma NN, et al. Initiation of sulphonylureas versus metformin is associated with higher blood pressure at one year. Pharmacoepidemiol Drug Saf. 2012; 21(5):515-23.

37. Huizinga $\mathrm{MM}$, Roumie $\mathrm{CL}$, Greevy RA, et al. Glycemic and weight changes after persistent use of incident oral diabetes therapy: a Veterans Administration retrospective cohort study. Pharmacoepidemiol Drug Saf. 2010;19(11):1108-12.

38. Rasool S, Kadla SA, Rasool V, et al. A comparative overview of general risk factors associated with the incidence of colorectal cancer. Tumour Biol. 2013;34(5):2469-76.

39. Ford ES, Malarcher AM, Herman WH, et al. Diabetes mellitus and cigarette smoking. Findings from the 1989 National Health Interview Survey. Diabetes Care. 1994;17(7): 688-92.

40. Joseph J, Svartberg J, Njølstad I, et al. Incidence of and risk factors for type-2 diabetes in a general population: the Troms $\varnothing$ Study. Scand J Public Health. 2010;38(7): 768-75.

41. Wolkin KY, Yan Y, Colditz GA, et al. Physical activity and colon cancer prevention: a metaanalysis. Br J Cancer. 2009;100(4):611-6. 



\section{Chapter}

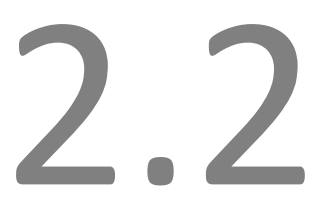

\section{Use of incretin agents and risk of pancreatic}

cancer: a population-based cohort study

L.M. Knapen, J. van Dalem, Y.C. Keulemans, N.P. van Erp, M.T. Bazelier, M.L. De Bruin, H.G.M. Leufkens, S. Croes, C. Neef, F. de Vries, J.H.M. Driessen

Diabetes Obes Metab. 2016;18(3):258-65 


\section{Abstract}

\section{Background / objectives}

To investigate the association between the use of incretin agents and the risk of pancreatic cancer.

\section{Patients and methods}

A retrospective population-based cohort study, using data from the Clinical Practice Research Datalink, 2007-2012, was conducted. Patients $(n=182,428)$ with at least one non-insulin antidiabetic drug (NIAD) prescription and aged $\geq 18$ during data collection, were matched one-to-one to control patient without diabetes. Multivariable Cox proportional hazards models and a 'new user design' were used to estimate the hazard ratio (HR) of pancreatic cancer in incretin users $(n=28,370)$ compared with control subjects without diabetes and other NIADtreated patients. Time-dependent adjustments were made for age, sex, lifestyle, comorbidities and drug use.

\section{Results}

The mean duration of follow-up was 4.1 years for incretin users. Current NIAD use was associated with a fourfold increased risk of pancreatic cancer (HR 4.28; $95 \%$ confidence interval (Cl) 3.49-5.24). This risk was almost doubled among current incretin users as compared with control subjects. Incretin use was not associated with pancreatic cancer when compared with control subjects with diabetes (HR 1.36; 95\% Cl 0.94-1.96); however, the 'new user design' did show an association between incretin use and pancreatic cancer when compared with control subjects with diabetes. In both cohorts with prevalent and incident users of antidiabetic drugs, the risk of pancreatic cancer almost doubled in those who had recently initiated incretin therapy (up to seven prescriptions), whereas this elevated risk dropped to baseline levels with prolonged use.

\section{Conclusions}

We found that incretin use was not associated with pancreatic cancer after adjustment for the severity of the underlying type 2 diabetes mellitus (T2DM). The elevated risk of pancreatic cancer in those recently initiating incretin agents is likely to be caused by protopathic bias or other types of unknown distortion. The presence of considerable confounding by disease severity and the lack of a duration-of-use relationship do not support a causal explanation for the association between incretin agents and pancreatic cancer. 


\section{Introduction}

Type 2 diabetes mellitus (T2DM) has become a major threat to human health. Almost $90 \%$ of T2DM patients fail to achieve target values for glucose, lipids, and blood pressure while treated with a non-insulin antidiabetic drug (NIAD) or insulin ${ }^{1}$. Incretin agents [glucagon-like peptide-1 receptor agonists (GLP-1RAs), such as exenatide or liraglutide, and dipeptidyl peptidase-4 inhibitors (DPP4-Is), such as saxagliptin, or sitagliptin] are new therapeutic agents for the treatment of T2DM. Incretin agents have a sustained antihyperglycaemic effect, while promoting weight loss with a minimal risk of hypoglycaemia ${ }^{2}$; however, evidence has arisen that pancreatic cancer is an important potential side effect of incretin agents ${ }^{2}$. Spontaneous adverse event reporting systems have detected cases of pancreatitis in users of incretin agents ${ }^{3}$. Moreover, animal models showed that incretin agents can lead to alterations associated with pancreatic cancer, such as inflammation, chronic pancreatic damage, inhibition of apoptosis and proliferation in 6 -cells, proliferation of pancreatic acinar and ductal cells and increased pancreatic mass ${ }^{2,4-10}$. A post-mortem clinical study showed an average $40 \%$ increase in pancreas mass in American organ donors who had been treated with incretins versus other antidiabetic drugs ${ }^{10}$; however, randomised controlled trials (RCTs) and a meta-analysis of RCTs ( $n=29,598$, follow-up 0.23-2.1 years) did not show an increased risk of pancreatic cancer ${ }^{11-13}$. Furthermore, no association between the use of incretin agents and pancreatic cancer was found in a large observational cohort study [DPP4-I and thiazolidinedione treatment cohort, $n=29,366$; DPP4-I and sulphonylurea derivative treatment cohort, $n=18,179$; follow-up period 5-18 months $]^{14}$. These RCTs were restricted, however, to patients with cardiovascular disease or the elderly, whereas incretins are also used by other groups of patients ${ }^{15}$.

Most studies did not evaluate the association between pancreatic cancer and GLP-1RAs or the widely prescribed DPP4-Is vildagliptin and linagliptin. Moreover, the results were not stratified according to the duration of drug use which could shed more light on the plausibility of the underlying aetiology ${ }^{11-13}$. The aim of the present study, therefore, was to assess the risk of pancreatic cancer in patients with T2DM who were using incretin agents (GLP-1RAs and DPP4-Is) compared with patients with T2DM using other NIADs and with population-based control subjects without diabetes mellitus.

\section{Materials and methods}

Data for this study were obtained from the United Kingdom (UK) Clinical Practice Research Datalink (CPRD), previously known as the General Practice Research Database (http://www.CPRD.com). The CPRD contains computerised medical records of 625 primary care practices in the UK, representing $8 \%$ of the population. The data 
recorded in the CPRD include demographic information, prescription details, clinical events, preventive care provided, specialist referrals, hospital admissions and major outcomes since 1987. Previous studies using CPRD data have shown a high validity with regard to a wide range of diseases. Moreover, CPRD data have previously been used to study a wide range of malignancies, including pancreatic cancer ${ }^{16-18}$.

We conducted a retrospective population-based cohort study largely according to methods that have been described previously ${ }^{15}$. All patients aged $\geq 18$ years at start of follow-up, with at least one NIAD prescription during the period of valid CPRD data collection were included in the study population. The study period started on 13 June 2007 (the date of the first recorded prescription of an incretin agent in the CPRD) and ended on 31 August 2012. The index date was defined as the date of the first NIAD prescription after the practice started to contribute data delivery to CPRD.

Each NIAD user was matched by sex, year of birth (within 5 years) and practice to one control subject who never received a NIAD or insulin prescription during follow-up. The index date of each control subject was set to the index date of his or her matched NIAD user. Their period of follow-up was divided into 90-day intervals. Each subject was then followed from his or her index date up to the end of data collection, the date of transfer out of the practice area, or the subject's death, or the earliest record of pancreatic cancer, i.e. the outcome of interest, whichever came first.

Follow-up time was divided into intervals based on the NIAD and incretin prescriptions, i.e. for every prescription a new interval was created. Exposure of NIAD users was defined as follows: after a washout period of 90 days, an interval was classified as 'past NIAD use', until the end of follow-up or a new prescription of an antidiabetic drug, whichever came first. Otherwise an interval was classified as 'current NIAD use'.

The NIAD users could move between current and previous exposure over time. Current NIAD users were further stratified by their exposure status to incretin agents and other non-incretin NIADs. Incretin use was further stratified by current GLP-1RA use and DPP4-I use. Current, recent and past incretin use were defined as GLP-1RA/DPP4-I use 0-90, 91-180 and >180 days before the start of an interval, respectively. Recent and past users became current users again with a new GLP-1RA/DPP4-I prescription. To evaluate duration of incretin use, current users were stratified by the number of incretin prescriptions ever before (in the UK, a single incretin prescription is generally issued every 28 days in case of chronic use).

Each patient was followed until the first event of pancreatic cancer, which was classified by the use of read codes that were reviewed by a gastroenterologist (Y.K.). Operational definitions are shown in Table 2.2.1. All patients with a history of pancreatic cancer were excluded as well as patients with polycystic ovaries or polycystic ovarian syndrome before the start of follow-up because metformin may be used as a treatment for these conditions. 
Table 2.2.1 Identification of pancreatic cancer by read codes.

\begin{tabular}{|c|c|c|c|}
\hline Medcode & $\begin{array}{l}\text { Clinical } \\
\text { events }\end{array}$ & Readcode & Readterm \\
\hline 8166 & 12138 & B170.00 & Malignant neoplasm of pancreas \\
\hline 16931 & 1821 & B80Z000 & Carcinoma in situ of pancreas \\
\hline 8771 & 853 & B170.00 & Malignant neoplasm of head of pancreas \\
\hline 34388 & 884 & B17Z.00 & Malignant neoplasm of pancreas NOS \\
\hline 35535 & 95 & B173.00 & Malignant neoplasm of pancreatic duct \\
\hline 35795 & 6 & B174.00 & Malignant neoplasm of Islets of Langerhans \\
\hline 39870 & 90 & B172.00 & Malignant neoplasm of tail of pancreas \\
\hline 40810 & 97 & B171.00 & Malignant neoplasm of body of pancreas \\
\hline 48537 & 6 & B17Y.00 & Malignant neoplasm of other specific sites of pancreas \\
\hline 49629 & 7 & BB5C100 & [M] Gastrinoma, malignant \\
\hline 61764 & 6 & BB5Y100 & [M] Vipoma \\
\hline 63102 & 6 & BB5B100 & [M] Islet cell carcinoma \\
\hline 95783 & 9 & B17YZ00 & Malignant neoplasm of specified site of pancreas NOS \\
\hline 96635 & 2 & B17Y000 & Malignant neoplasm of ectopic pancreatic tissue \\
\hline 97875 & 1 & B175.00 & Malignant neoplasm, overlapping lesion of pancreas \\
\hline 98825 & 2 & BB5B600 & [M] Mixed islet cell and exocrine adenocarcinoma \\
\hline
\end{tabular}

NOS: not otherwise specified.

The presence of potential confounders was assessed by reviewing the computerised medical records for any evidence of these risk factors before the start of an interval. The following potential confounders were considered as general risk factors and were determined at baseline: sex, body mass index (BMI), smoking status and alcohol use. Other confounders considered in this study were determined time-dependently (i.e. at the start of each new interval): $\operatorname{age}^{19}$, gallstones/endoscopic retrograde cholangiopancreatography procedure $(E R C P)^{20,21}$ or alcoholism ${ }^{19,22}$. Alcoholism was defined as history of specific drugs used to treat alcoholism or a diagnosis of alcoholism. In addition, the following drug prescriptions 6 months before the start of an interval were considered to be potential confounders: paracetamol ${ }^{23}$; antibiotics (co-trimoxazole/macrolides/tetracyclines) ${ }^{24,25}$; angiotensin-converting enzyme (ACE) inhibitors ${ }^{24,25}$; loop diuretics ${ }^{24,25}$; statins $^{24,25}$; proton pump inhibitors ${ }^{24,25}$; or systemic glucocorticoids ${ }^{23,25}$. The following potential confounders for disease severity were considered time-dependently: a history of retinopathy and neuropathy ${ }^{23,25,26}$ and the most recent glycosylated hemoglobin type $\mathrm{A} 1 \mathrm{c}(\mathrm{HbA} 1 \mathrm{c})$ value in the year preceding the start of an interval.

We estimated the adjusted hazard ratio (HR) of pancreatic cancer among current NIAD users versus controls and among current incretin users versus other NIAD users using time-varying Cox proportional hazards regression (SAS 9.2; PHREG procedure). Potential confounders and indicators of disease severity were included in the final model if they independently changed the 8 -coefficient for the exposure of interest by at least $5 \%$, or when a consensus about inclusion existed within the team of researchers, supported by clinical evidence from literature. A sensitivity analysis 
repeated the main analysis in a 'new user design'27. Only patients who had started NIADs after 13 June 2007 were included. An extra sensitivity analysis was performed in order to exclude all women with a record of gestational diabetes during follow-up, as metformin may be used as a treatment for this condition. Furthermore, an extra sensitivity analysis was performed adjusting the main analysis for insulin use as well as a sensitivity analysis for history of pancreatitis, and use of calcium channel blockers and angiotensin receptor blockers in the previous 6 months.

\section{Results}

The study population consisted of 28,370 incretin users and 182,428 NIAD users, who were matched with 210,798 control subjects without diabetes. The mean duration of follow-up was 4.1 years for incretin users, 3.3 years for other NIAD users and 3.3 years for control subjects. The mean duration of incretin use was 1.18 years. Of the incretin users, $43.7 \%$ were female and their mean age at index date was 58.1 years. At baseline, the mean age of incretin users was 4 years younger than users of other NIADs, and they had a higher mean BMI. The severity of the underlying diabetes mellitus was higher among incretin users compared with other NIAD users as their most recently recorded mean $\mathrm{HbA1C}$ measurement in the preceding 12 months was $8.7 \%$ higher. With the exception of exposure to ACE inhibitors, statins or various antidiabetic drugs classes, there were no notable differences in history of comorbidities with incretin users versus other NIAD users at baseline (Table 2.2.2).

Table 2.2.2 Baseline characteristics of incretin users, other non-insulin antidiabetic drug users and controls ${ }^{\mathrm{a}}$.

\begin{tabular}{lccc}
\hline Characteristic & $\begin{array}{c}\text { Incretin users } \\
(\mathrm{N}=28,370)\end{array}$ & $\begin{array}{c}\text { Other NIAD users } \\
(\mathrm{N}=182,428)\end{array}$ & $\begin{array}{c}\text { Controls } \\
(\mathrm{N}=210,798)\end{array}$ \\
\hline Females & $12,410(43.7)$ & $86,000(47.1)$ & $98,410(46.7)$ \\
Mean duration of follow-up (years, SD) & $4.1(1.5)$ & $3.3(1.8)$ & $3.3(1.8)$ \\
Median duration of follow-up (years, IQR) & $5.1(2.1)$ & $3.7(3.4)$ & $4.0(3.1)$ \\
Age & & & \\
Mean age at index date (years, SD) & $58.1(11.8)$ & $62.4(14.9)$ & $61.8(14.6)$ \\
18 - 49 years & $6,746(23.8)$ & $35,585(19.5)$ & $42,331(20.1)$ \\
$50-59$ years & $8,319(29.3)$ & $34,764(19.1)$ & $43,083(20.4)$ \\
$60-69$ years & $8,359(29.5)$ & $47,650(26.1)$ & $56,009(26.6)$ \\
$>70$ years & $4,946(17.4)$ & $64,429(35.3)$ & $69,375(32.9)$ \\
BMI at index date & & & \\
Mean BMI at index date $\left(\mathrm{kg} / \mathrm{m}^{2}, \mathrm{SD}\right)$ & $33.6(7.1)$ & $31.1(6.5)$ & $26.8(5.1)$ \\
$<25.0 \mathrm{~kg} / \mathrm{m}^{2}$ & $2,180(7.7)$ & $26,648(14.6)$ & $72,236(34.3)$ \\
$25.0-29.9 \mathrm{~kg} / \mathrm{m}^{2}$ & $7,243(25.5)$ & $59,489(32.6)$ & $74,047(35.1)$ \\
$30.0-34.9 \mathrm{~kg} / \mathrm{m}^{2}$ & $8,462(29.8)$ & $50,309(27.6)$ & $29,927(14.2)$ \\
$\geq 35.0 \mathrm{~kg} / \mathrm{m}^{2}$ & $10,293(36.3)$ & $41,014(22.5)$ & $12,095(5.7)$ \\
Missing & $192(0.7)$ & $4,968(2.7)$ & $22,493(10.7)$ \\
\hline
\end{tabular}


Table 2.2.2 (continued)

\begin{tabular}{|c|c|c|c|}
\hline Characteristic & $\begin{array}{c}\text { Incretin users } \\
(\mathrm{N}=28,370)\end{array}$ & $\begin{array}{l}\text { Other NIAD users } \\
\quad(\mathrm{N}=182,428)\end{array}$ & $\begin{array}{c}\text { Controls } \\
(\mathrm{N}=210,798)\end{array}$ \\
\hline \multicolumn{4}{|l|}{ Smoking status } \\
\hline Never & $13,897(49.0)$ & $90,786(49.8)$ & $110,907(52.6)$ \\
\hline Current & 5,935 (20.9) & $35,823(19.6)$ & $43,821(20.8)$ \\
\hline Ex & $8,505(30.0)$ & $54,780(30.0)$ & $50,490(24.0)$ \\
\hline Missing & $33(0.1)$ & $1,039(0.6)$ & $5,580(2.6)$ \\
\hline \multicolumn{4}{|l|}{ Alcohol use } \\
\hline Yes & $19,297(28.6)$ & $118,957(29.0)$ & $38,090(18.1)$ \\
\hline No & $8,107(68.0)$ & $52,935(65.2)$ & $148,979(70.7)$ \\
\hline Missing & $966(3.4)$ & $10,536(5.8)$ & $23,729(11.3)$ \\
\hline Alcoholism & 533 (1.9) & $3,961(2.2)$ & 4,105 (1.9) \\
\hline \multicolumn{4}{|l|}{ History of comorbidities } \\
\hline Gallstones & $1,465(5.2)$ & $9,031(5.0)$ & $6,455(3.1)$ \\
\hline ERCP & $162(0.6)$ & $1,302(0.7)$ & $897(0.4)$ \\
\hline Retinopathy & $3,768(13.3)$ & $22,184(12.2)$ & $758(0.4)$ \\
\hline Neuropathy & $2,128(7.5)$ & $14,047(7.7)$ & $2,492(1.2)$ \\
\hline \multicolumn{4}{|c|}{ Drug use within six months before } \\
\hline Metformin & $15,099(53.2)$ & 67,087 (36.8) & $\mathrm{n} / \mathrm{a}$ \\
\hline Sulfonylurea derivatives & $8,156(28.7)$ & $31,812(17.4)$ & $\mathrm{n} / \mathrm{a}$ \\
\hline Thiazolidinediones & $5,481(19.3)$ & $13,899(7.6)$ & $\mathrm{n} / \mathrm{a}$ \\
\hline Insulin & $2,219(7.8)$ & $19,283(10.6)$ & $n / a$ \\
\hline Paracetamol & $7,170(25.3)$ & $47,502(26.0)$ & $38,093(18.1)$ \\
\hline ACE inhibitors & $10,826(38.2)$ & $64,848(35.5)$ & $30,733(14.6)$ \\
\hline Loop diuretics & $2,588(9.1)$ & 20,809 (11.4) & $10,330(4.9)$ \\
\hline Statins & $17,114(60.3)$ & $98,909(54.2)$ & $44,297(21.0)$ \\
\hline Proton pump inhibitors & $5,891(20.8)$ & $37,850(20.7)$ & $31,998(15.2)$ \\
\hline Systemic glucocorticoids & $1,086(3.8)$ & $9,387(5.1)$ & $6,791(3.2)$ \\
\hline \multicolumn{4}{|l|}{$\mathrm{HbA1c}$} \\
\hline$<6 \%$ & $435(1.5)$ & $6,490(3.5)$ & $2,381(1.1)$ \\
\hline $6-6.9 \%$ & 3,345 (11.7) & $26,968(14.7)$ & $2,122(1.0)$ \\
\hline $7-7.9 \%$ & $5,623(19.7)$ & 32,869 (17.9) & $438(0.2)$ \\
\hline $8-8.9 \%$ & 3,403 (11.9) & $16,986(9.3)$ & $222(0.1)$ \\
\hline$\geq 9 \%$ & $5,135(18.0)$ & $22,084(12.0)$ & $238(0.1)$ \\
\hline Missing & $10,598(37.1)$ & $78,213(42.6)$ & $206,748(97.5)$ \\
\hline
\end{tabular}

ACE: angiotensin-converting-enzyme; $\mathrm{BMI}$ : body mass index; $\mathrm{ERCP}$ : endoscopic retrograde cholangiopancreatography; HbA1c: glycosylated hemoglobin type A1c; IQR: interquartile range; n/a: not applicable; NIAD: non-insulin antidiabetic drug; SD: standard deviation. ${ }^{a}$ Data are $n$, (\%) unless stated otherwise.

Table 2.2.3 shows that current NIAD users had a fourfold higher risk of pancreatic cancer as compared with control subjects without diabetes (fully adjusted HR 4.28; 95\% $\mathrm{Cl}$ 3.49-5.24). This association almost doubled when the subgroup of current incretin users was compared with control subjects without diabetes (fully adjusted HR 7.52; 95\% Cl 5.09-11.12). There was no difference between current use of GLP-1RA or DPP4-Is and the risk of pancreatic cancer. 


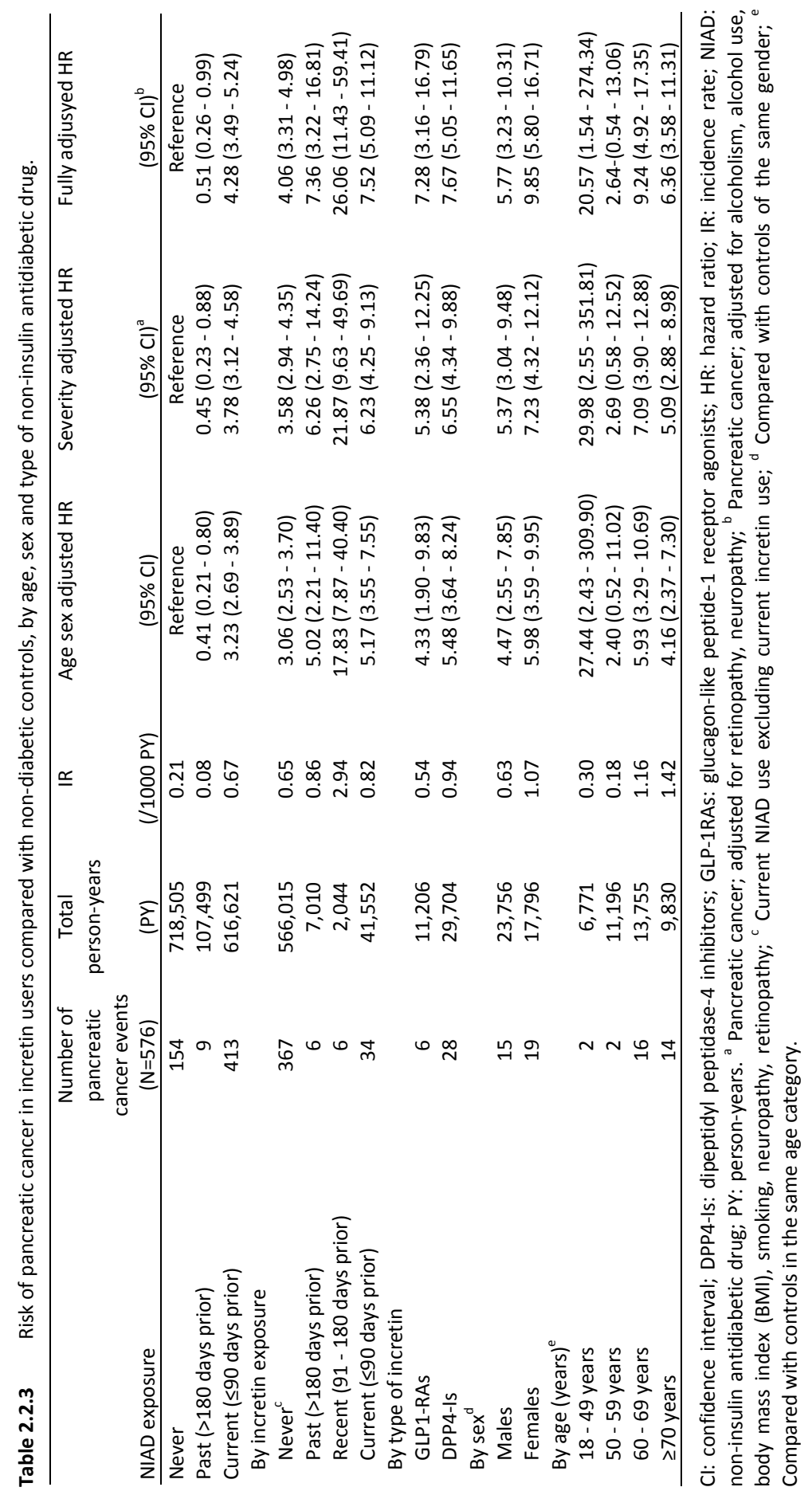


To adjust for confounding by indication, current incretin users were compared with other NIAD users. Results show a 1.7-fold increased risk of pancreatic cancer among current incretin users. This association decreased after adjustment for disease severity, yielding a fully adjusted HR of 1.27; ( $\mathrm{Cl} 0.88-1.83)$. Additional statistical adjustment for general risk factors did not substantially change this result (adjusted HR $1.36 ; 95 \% \mathrm{Cl}$ 0.94-1.96). The prolonged use of incretin agents did not further increase the risk of pancreatic cancer. Incretin users who had recently started taking the drugs (4-7 prescriptions) had an almost twofold higher risk (adjusted HR 1.86; $95 \% \mathrm{Cl}$ 1.01-3.42), whereas this elevated risk dropped to baseline levels with prolonged use (adjusted HR 0.95; 95\% Cl 0.53-1.72) (Table 2.2.4).

Table 2.2.5 shows the sensitivity analysis with a 'new user design', in which the cohort was restricted to starters of NIADs (including incretins). A statistically significant 1.7-fold increased risk for pancreatic cancer was found in current incretin users (HR 1.67; 95\% Cl 1.01-2.77) versus other NIAD users. Similar to the results in Table 2.2.4, this risk was more than twice as high among patients who had been prescribed up to 60 days of incretins (two prescriptions), whereas the risk dropped to baseline levels with long-term use ( 8 months, measured as prescriptions, exposure or more). In the extra sensitivity analysis in which women with gestational diabetes were excluded, we found that current incretin use was not associated with pancreatic cancer (adjusted HR 1.36; $95 \% \mathrm{Cl}$ 0.94-1.96). Furthermore, the main results of the extra sensitivity analysis showed that incretin use (adjusted HR 1.44; $95 \% \mathrm{Cl} 0.99-2.09$ ) as well as use of calcium channel blockers and angiotensin receptor blockers in the previous 6 months (adjusted HR 1.37; 95\% Cl 0.95-1.98) were not associated with pancreatic cancer.

\section{Discussion}

The present study found an 1.7 fold increased risk of pancreatic cancer with incretin use which disappeared after statistical adjustments for the severity of the underlying T2DM. In recent starters of incretin agents, the risk of pancreatic cancer was almost doubled and dropped to baseline levels with prolonged use. The presence of considerable confounding by disease severity and the lack of a duration of use relationship in our results do not support a causal relationship between incretin use and pancreatic cancer.

Our results are not consistent with the study by Elashoff et al. ${ }^{28,29}$. This study showed that pancreatic cancer was significantly more reported in patients treated with sitagliptin or exenatide as compared with users of other antidiabetic therapies ${ }^{28}$; however, that study provided hypothesis-generating evidence only, as it was based on data from the United States (US) Food and Drug Administration's spontaneous adverse event reporting system ${ }^{29}$. 


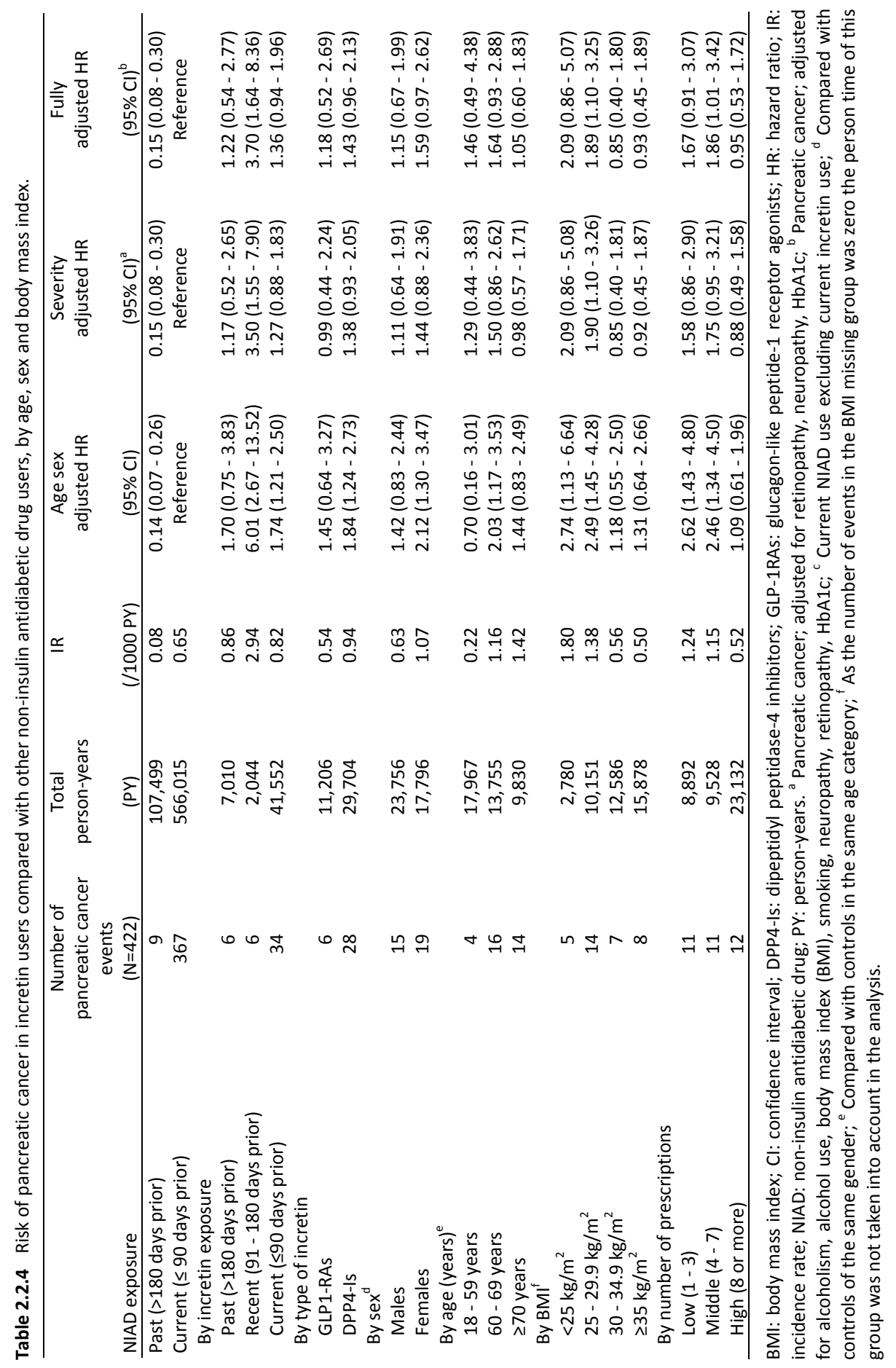




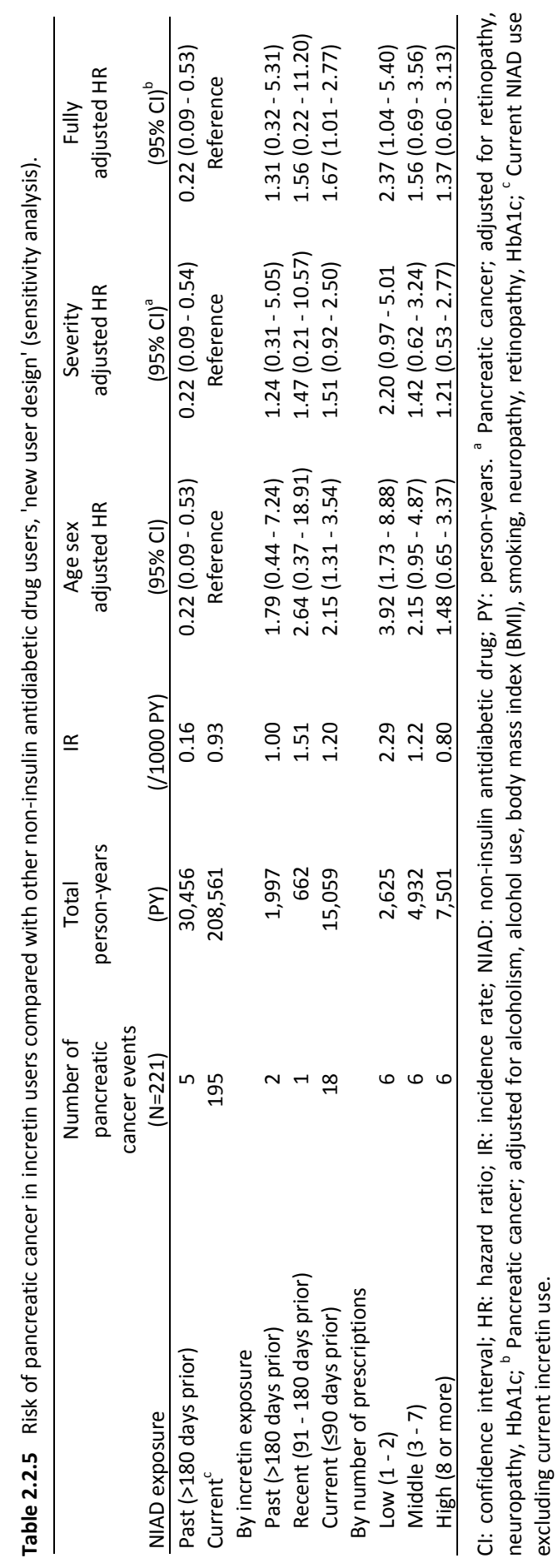


Several previous studies have reported results in line with our results, identifying no statistically significant risk of pancreatic cancer ${ }^{11-14}$. A meta-analysis of 25 RCTs (sitagliptin arm, $n=7,726$; comparator agent, $n=6,885$ ), with a follow-up period of 12-104 weeks, found no effect on pancreatic cancer occurrence ( 0.05 and 0.06 events per 100 patient-years in the sitagliptin and non-exposed group, respectively) ${ }^{11}$. In the SAVOR cardiovascular outcome trial ( $n=16,492$, median follow-up 2.1 years) there were five cases of pancreatic cancer in the saxagliptin arm and 12 cases in the comparator agent arm, while no events of pancreatic cancer were reported in the alogliptin or placebo arm of the EXAMINE cardiovascular outcome trial $(n=5,380$, median follow-up 18 months $)^{12,13}$. Furthermore, an observational cohort study using a US claims database did not show an association between risk of pancreatic cancer with DPP4-I use versus use of sulphonylurea derivatives or thiazolidinediones ${ }^{14}$.

The potential biological mechanisms of incretin agents promoting or enhancing pancreatic cancer are supported by limited indirect evidence. In animal models three GLP-1-induced pathways have been proposed; proliferation in B-cells, inhibition of $B$-cells, and enhanced differentiation of adult stem cells in the ductal pancreatic epithelium. This could lead to chronic pancreatic damage, inflammation of pancreatic acinar and ductal cells, increased formation of dysplastic pancreatic intraepithelial neoplasia lesions and an increase in pancreatic weight ${ }^{2,4,5,8,30-34}$. GLP-1RA-dependent effects on 6 -cell proliferation include activation of phosphatidylinositol-3 (PI3) kinase, protein kinase $B(A K T)$, mitogen-activated protein kinase (MAPK), protein kinase $C$, the src kinase, and the epidermal growth factor receptor $(E G F R)^{6,35,36}$; however, the exact mechanism by which GLP-1RA activates the PI3 kinase signalling pathway remains unknown ${ }^{6}$. Both EGFR and src have been implicated in the pathogenesis and progression of numerous malignant tumours, including pancreatic cancer ${ }^{35}$. Furthermore, duct cell proliferation and pancreatic intraepithelial neoplasia lesions might lead to duct occlusion, which could cause back pressure in the pancreas, stressing the acinar cells to release the digestive enzymes, with the resulting chronic pancreatitis fostering further development of pancreatic intraepithelial neoplasia lesions and duct cell proliferation ${ }^{5,34}$. By activating both above-mentioned pathways, incretin agents could promote acute pancreatitis, chronic pancreatitis and eventually development of pancreatic cancer ${ }^{34,35}$. Instead of inducing pancreatic cancer, it was hypothesised that incretin agents may enhance tumour growth which could lead to earlier detection. This underlying mechanism was also not confirmed by our data, including the duration of use analysis.

It is important to note that the duration of follow-up of the present study was relatively short (up to five years) in order to detect a causal effect of incretins on pancreatic cancer. The mean duration of incretin use was only 1.2 years. The time span from an initiated pancreatic intraepithelial lesion to a parental clone, which will initiate an infiltrating pancreatic carcinoma, is $\sim 12$ years ${ }^{37}$. 
Although a limited duration of follow-up and continuous use of incretins may not be supportive for an observational study to evaluate a causal relationship, it can give valuable information about the alternative hypothesis that the results may be flawed by unmeasured distortion. Other well-known examples include CPRD studies that have evaluated the association between hip fracture and the use of statins ${ }^{38}$ or proton pump inhibitors ${ }^{39}$. Similarly to the present study, the associations were strongest briefly after the initiation of statins or proton pump inhibitors, and then attenuated with prolonged use (the opposite of a causal hypothesis). The lack of an association between statin use and fracture risk was confirmed in various large $\mathrm{RCTs}^{38,39}$. Protopathic bias is a potential explanation for the rapidly increased risk of pancreatic cancer that we observed in the present study. Protopathic bias occurs when a pharmaceutical agent is inadvertently prescribed for an early manifestation of a disease that has not yet been diagnostically detected $^{40,41}$. It is well known that pancreatic cancer is a powerful diabetogenic state, as illustrated by prevalence of pancreatic cancer-associated T2DM varying from 4 to $64 \%^{39,42,43}$. T2DM was found to be more prevalent ( 47 versus $7 \% ; p<0.001$ ) and at onset ( $<2$ years; 74 versus $53 \% ; p=0.002$ ) among patients with pancreatic cancer compared with patients without pancreatic cancer ${ }^{43,44}$. Lifestyle interventions and use of antihyperglycaemic drugs are usually inadequate in treating this pancreatic cancerassociated T2DM, leading to rapid prescribing of antihyperglycaemic treatment such as incretin agents. This may be indirectly supported by the previously reported 1.7 -fold increased risk of pancreatic cancer with sulphonylurea derivative use ${ }^{45}$, and the fourfold increased risk of pancreatic cancer with NIAD treatment observed in the present study.

In addition to the presence of protopathic bias and confounding by disease severity, it is likely that our observed associations are not without residual confounding. For instance, we were not able to correct for the amount of exercise. Physical activity appears to decrease the risk of pancreatic cancer, especially among overweight people $^{46}$. Incretin users might perform less exercise compared with non-incretin users, which could lead to an overestimation of our effect.

The present study has several strengths. We were able to adjust statistically in a time-dependent way for several potentially important confounders, including age, the most recently recorded $\mathrm{HbA} 1 \mathrm{c}$ value in the preceding year, and different comorbidities, such as retinopathy, neuropathy, alcoholism and drug use. Additionally, we had information at baseline on sex, smoking status, BMI and alcohol use for almost all patients. Furthermore, CPRD data are collected prospectively, eliminating the risk of recall bias. Finally, a substantial amount of data representing the general population of the UK was available. It is important to note, however, that the incidence rates of pancreatic cancer in the present study were slightly higher compared with known rates in the UK population ${ }^{47}$. We observed a rate of 21 per 100,000 person-years for patients without T2DM and 67 per 100,000 person-years for patients with T2DM. This is possibly attributable to the characteristics of the study cohort, which included only patients 
aged $\geq 18$ years (i.e. it excluded children, who represent $\sim 20 \%$ of the UK population), and also to the fact that the control cohort had the same age and gender distribution as the T2DM cohort as a result of matching. Given that age is an important risk factor for pancreatic cancer, the average incidence rates would be expected to be higher.

In conclusion, we found that incretin use was not associated with pancreatic cancer after adjustment for indicators of the severity of the underlying T2DM. The elevated risk of pancreatic cancer found in recent starters of incretin agents may be the result of protopathic bias or other types of unknown distortion. The presence of considerable confounding by disease severity and the lack of duration of use relationship do not support a causal explanation for the observed association between incretin agents and pancreatic cancer. A longer duration of follow-up and exposure to incretin agents is required to investigate the association between exposure time to incretin agents and pancreatic cancer. 


\section{References}

1. Drucker DJ, Sherman SI, Gorelick FS, et al. Incretin-Based Therapies for the Treatment of Type 2 Diabetes: Evaluation of the Risks and Benefits. Diabetes Care. 2010;33(2):428-33.

2. Butler PC, Elashoff $M$, Elashoff $R$, et al. $A$ critical analysis of the clinical use of incretinbased therapies: are the GLP-1 therapies safe? Diabetes Care. 2013;36:2118-25.

3. Raschi E, Piccinni C, Poluzzi E, et al. The association of pancreatitis with antidiabetic drug use: gaining insight through the FDA pharmacovigilance database. Acta Diabetol. 2013;50(4):569-77.

4. Farilla L, Hui H, Bertolotto $C$, et al. Glucagonlike peptide-1 promotes islet cell growth and inhibits apoptosis in Zucker diabetic rats. Endocrinology. 2002;143(11):4397-408.

5. Ryder RE. The potential risks of pancreatitis and pancreatic cancer with GLP-1-based therapies are far outweighed by the proven and potential (cardiovascular) benefits. Diabet Med. 2013;30(10):1148-55.

6. Buteau J, Foisy S, Joly E, et al. Glucagon-Like Peptide 1 Induces Pancreatic $\beta$-cell Growth Factor Receptor. Diabetes. 2003; 52(1):12432.

7. Niebisz-Cieślak AB, Karnafel W. Insulin sensitivity in chronic pancreatitis and features of insulin resistance syndrome. Pol Arch Med Wewn. 2010;120(7-8):255-63.

8. Cohen D. Pressure mounts for companies to hand over data on antidiabetes drugs linked to pancreatic harm. BMJ. 2013;346:f3900.

9. Shyangdan DS, Royle PL, Clar C, et al. Glucagon-like peptide analogues for type 2 diabetes mellitu: systematic review and metaanalysis. BMC Endocr Disord. 2010;10:20.

10 Butler AE, Campbell-Thompson M, Gurlo T, et al. Marked expansion of exocrine and endocrine pancreas with incretin therapy in humans with increased exocrine pancreas dysplasia and the potential for glucagonproducing neuro-endocrine tumors. Diabetes. 2013;62(7):2595-604.

11. Engel SS, Round E, Golm GT, et al. Safety and tolerability of sitagliptin in type 2 diabetes: pooled analysis of 25 clinical studies. Diabetes Ther. 2013;4:119-45.

12. Scirica BM, Bhatt DL, Braunwald E, et al. Saxagliptin and cardiovascular outcomes in patients with type 2 diabetes mellitus. N Engl J Med. 2013; 369:1317-26.

13. White WB, Cannon CP, Heller SR, et al. Alogliptin after acute coronary syndrome in patients with type 2 diabetes. N Engl J Med. 2013;369:1327-35.

14. Gokhale M, Buse JB, Gray CL, et al. Dipeptidylpeptidase-4 inhibitors and pancreatic cancer: a cohort study. Diabetes Obes Metab. 2014;16(12):1247-56.

15. Driessen JH, van Onzenoort HA, Henry RM, et al. Use of dipeptidyl peptidase-4 inhibitors for type 2 diabetes mellitus and risk of fracture. Bone. 2014;68:124-30.

16. Herrett E, Thomas SL, Schoonen WM, et al. Validation and validity of diagnoses in the General Practice Research Database: a systematic review. Br J Clin Pharmacol. 2010; 69(1):4-14.

17. Lalmohamed A, MacGregor AJ, de Vries F, et al. Patterns of risk of cancer in patients with metal-on-metal hip replacements versus other bearing surface types: a record linkage study between a prospective joint registry and general practice electronic health records in England. PLoS One. 2013;8(7):e65891.

18. Bodmer M, Becker C, Meier C, et al. Use of antidiabetic agents and the risk of pancreatic cancer: a case-control analysis. Am J Gastroenterol. 2012;107(4):620-6.

19. Zhang J, Dhakal I, Ning B, et al. Patterns and trends of pancreatic cancer mortality rates in Arkansas, 1969-2002: a comparison with the US population. Eur J Cancer Prev. 2008; 17(1):18-27.

20. Nogueira L, Freedman ND, Engels EA, et al. Gallstones, cholecystectomy, and risk of digestive system cancers. Am J Epidemiol. 2014;179(6):731-9.

21. Patai Á, Patai ÁV, Solymosi N, et al. Prevention of acute pancreatitis following endoscopic retrograde cholangio-pancreatography. Orv Hetil. 2015;156(18):715-9.

22. Herreros-Villanueva M, Hijona E, Bañales JM, et al. Alcohol consumption on pancreatic diseases. World J Gastroenterol. 2013;19(5): 638-47.

23. Vinklerová $\mathrm{I}$, Procházka $\mathrm{M}$, Procházka, $\mathrm{V}$, et al. Incidence, severity, and etiology of druginduced acute pancreatitis. Dig Dis Sci. 2010;55(10):2977-81. 
24. Kaurich T. Drug-induced acute pancreatitis. Proc (Bayl Univ Med Cent). 2008;21(1):77-81.

25. Trivedi CD, Pitchumoni CS. Drug-induced pancreatitis: an update. J Clin Gastroenterol. 2005;39(8):709-16.

26. Urbánek K, Vinklerová I, Krystyník O, et al. Acute Pancreatitis Induced by Drugs, Acute Pancreatitis, Prof. Luis Rodrigo (Ed.), ISBN: 978-953-307-984-4. Retrieved from http://www.intechopen.com/books/acutepancreatitis/acute-pancreatitis-induced-bydrugs. Accessed April 2015.

27. Ray WA. Evaluating medication effects outside of clinical trials: new-user designs. Am J Epidemiol. 2003;158(9):915-20.

28. Elashoff M, Matveyenko AV, Gier B, et al. Clinical-Pancreas. Pancreatitis, Pancreatic, and Thyroid Cancer With Glucagon-Like Pepide-1Based Therapies. Gastroenterology. 2011;141: 150-6.

29. Ben $Q, X u M, N i n g ~ X$, et al. Diabetes mellitus and risk of pancreatic cancer: A meta-analysis of cohort studies. Eur J Cancer. 2011;47(13): 1928-37.

30. Singh S, Chang HY, Richards TM, et al. Glucagon-like peptide 1-based therapies and risk of hospitalization for acute pancreatitis in Type 2 diabetes mellitus: a population-based matched case-control study. JAMA Intern Med. 2013;173:534-9.

31. Yu X, Tang H, Huang L, et al. Exentatideinduced chronic damage of pancreatic tissue in rats. Pancreas. 2012;41(8):1235-40.

32. Nachnani JS, Bulchandani DG, Nookala A, et al. Biochemical and histological effects of exendin-4 (exenatide) on the rat pancreas. Diabetologia. 2010;53(1):153-9.

33. Rolin B, Larsen MO, Gotfredsen CF, et al. The long-acting GLP-1 derivative NN2211 ameliorates glycemia and increases beta-cell mass in diabetic mice. Am J Physiol Endocrinol Metab. 2002;283(4):45-52.

34. Gier B, Matveyenko AV, Kirakossian D, et al. Chronic GLP-1 receptor activation by exendin4 induces expansion of pancreatic duct glands in rats and accelerates formation of dysplastic lesions and chronic pancreatitis in the Kras(G12D) mouse model. Diabetes. 2012; 61(5):1250-62.

35. Spranger J, Gundert-Remy U, Stammschulte T. GLP-1-based therapies: the dilemma of uncertainty. Gastroenterology. 2011;141(1): 20-3.
36. Buteau J. GLP-1 receptor signaling: effects on pancreatic beta-cell proliferation and survival. Diabetes Metabolism. 2008;34;Suppl2:S73-7.

37. Yachida S, Jones S, Bozic I, et al. Distant metastasis occurs late during the genetic evolution of pancreatic cancer. Nature. 2010;467(7319):1114-7.

38. de Vries F, de Vries C, Cooper C, et al. Reanalysis of two studies with contrasting results on the association between statin use and fracture risk: the General Practice Research Database. Int J Epidemiol. 2006;35(5):1301-8.

39. de Vries F, Cooper AL, Cockle SM, et al. Fracture risk in patients receivingacidsuppressant medication alone and in combination with bisphosphonates. Osteoporos Int. 2009;20(12):1989-98.

40. Horwitz RI, Feinstein AR. The problem of protopathic bias in case-control studies. Am J Med. 1980;68(2):255-8.

41. De Bruijn KM, Ruiter R, de Keyser CE, et al. Detection bias may be the main cause of increased cancer incidence among diabetics: results from the Rotterdam Study. Eur J Cancer. 2014;50(14):2449-55.

42. Roberts SE, Akbari A, Thorne K, et al. The incidence of acute pancreatitis: impact of social deprivation, alcohol consumption, seasonal and demographic factors. Aliment Pharmacol Ther. 2013;38(5):539-48.

43. Pannala R, Leirness JB, Bamlet $W R$, et al. Prevalence and clinical profile of pancreatic cancer-associated diabetes mellitus. Gastroenterology. 2008;134(4):981-7.

44. Halfdanarson TR, Pannala R. Incretins and risk of neoplasia. BMJ. 2013;346:f3750.

45. Singh S, Singh PP, Singh AG, et al. Anti-diabetic medications and risk of pancreatic cancer in patients with diabetes mellitus: a systemic review and meta analysis. Am J Gastroenterol. 2013;108(4):510-9.

46. Michaud DS, Giovannucci E, Willett WC, et al. Physical activity, obesity, height, and the risk of pancreatic cancer. JAMA. 2001;286(8):9219.

47. Cancer research UK. Pancreatic Cancer Incidence Rates. Retrieved from http://www.cancerresearchuk.org/healthprofessional/cancer-statistics/statistics-bycancer-type/pancreatic-cancer/incidence\# heading-One. Accessed November 2015. 

0

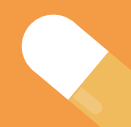

$\theta$

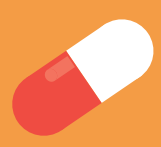

$\theta$

○ 


\section{Chapter 3}

Effectiveness of targeted oncologic therapies in real-life populations 


\section{Chapter}

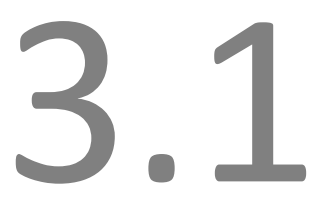

A real-life study on the implementation and effectiveness of exemestane plus everolimus per hospital type in patients with advanced breast cancer. A study of the Southeast Netherlands advanced breast cancer registry

L.M. Knapen, S.M.E. Geurts, K.I.E. Ibragimova, S. Croes, B.E.P.J. Vriens, F.W.P.J. van den Berkmortel, M.W. Dercksen, A.J. van de Wouw, M.J.A.E. Pepels, A.O.J. de Fallois, D.J.A. Lobbezoo, M. de Boer, V.C.G. Tjan-Heijnen

Breast. 2019;44:46-51 


\section{Abstract}

\section{Background / objectives}

We aimed to assess the implementation and effectiveness of exemestane plus everolimus treatment per hospital type in real-life, shortly after approval of everolimus.

\section{Patients and methods}

Advanced breast cancer patients treated with exemestane plus everolimus in 2012-2014 were included from the SONABRE registry. Progression-free survival (PFS) and a 12-week conditional PFS (post-hoc) were estimated by Kaplan-Meier method. The multivariable Cox proportional hazards model was performed by type of hospital and adjusted for patient, tumour and treatment characteristics.

\section{Results}

We included 122 patients, comprising 48 patients treated in academic $(n=1), 56$ in teaching $(n=4)$, and 18 in non-teaching $(n=2)$ hospitals. The median PFS was 6.3 months (95\% confidence interval (Cl) 4.0-8.6) overall, and 8.5 months (95\% Cl 7.7-9.3), 4.2 months (95\% Cl 2.0-6.3), and 5.5 months $(95 \% \mathrm{Cl}$ 4.2-6.7) for the patients treated in academic, teaching and non-teaching hospitals, respectively. The adjusted hazard ratio (HR) for PFS-events was $1.5(95 \% \mathrm{Cl} 1.0-2.2)$ and 1.0 (95\% Cl 0.5-1.9) respectively for patients treated at teaching and non-teaching hospitals versus the academic hospital. The adjusted HR for 12-week conditional PFS-events was not different between hospital types. In the first 12-week treatment period, treatment was discontinued due to early progression in one out of 48 patients in the academic versus nine out of 74 patients in the non-academic hospitals, confirmed by imaging in one and two patients, respectively.

\section{Conclusions}

In our study, the median PFS was borderline significantly different between hospital types, possibly the result of a different assessment approach in the first 12-week treatment period. 


\section{Introduction}

Advanced breast cancer $(A B C)$ is a major cause of death among women worldwide. The median overall survival (OS) of patients diagnosed with $A B C$ irrespective of their breast cancer subtype is $\sim 2-3$ years and the 5 -year survival is only $\sim 25 \%{ }^{1}$. The hormone receptor-positive/human epidermal growth factor receptor 2-negative (HR-positive/HER2-negative) subtype is the leading subtype of $A B C$, accounting for $60-70 \%$ of all patients with $A B C^{2}$. In recent years, progress has been made in the treatment of these patients by the introduction of new therapeutic drugs ${ }^{3,4}$. Everolimus, an oral inhibitor of the mammalian target of rapamycin (mTOR) that targets the oncogenic PI3K-AKT-mTOR driver pathway, is one of these agents ${ }^{3,5}$.

Everolimus in combination with exemestane was evaluated in the BOLERO-2 trial in patients who had recurrence or progression on a non-steroidal aromatase inhibitor ${ }^{3}$. Progression-free survival (PFS) was significantly longer for exemestane plus everolimus as compared with exemestane plus placebo, with a median of 7.8 months versus 3.2 months (hazard ratio (HR) 0.45$)^{6}$. No statistically significant difference in OS was detected (31.0 months for exemestane plus everolimus versus 26.6 months for exemestane alone; HR 0.89$)^{7}$. It should be noted, however, that there was a small imbalance in post-study use of chemotherapy (53\% versus $63 \%$, respectively), which may partly explain the lack of an improved OS besides a lack of power for this secondary study endpoint ${ }^{7}$. The results of the BOLERO-2 phase 3 trial were in line with previous phase 2 trials ${ }^{5}$.

Randomised controlled trials offer the highest level of evidence on efficacy of a particular treatment, but in real-life the situation may be different. The use of a drug in a population with generally more comorbidities than in a trial setting may lead to more dose reductions or earlier treatment cessation. Ultimately, patient selection and delivered treatment modifications impact effectiveness, as shown in previous phase IIIb studies on everolimus ${ }^{8-10}$. In addition, the implementation of new anti-cancer drugs in real-life is often slow and a large variation between different hospital types has actually been shown for a number of systemic therapies ${ }^{11,12}$. For that reason real-life data may not only support future reimbursement decisions and shared decision making in real-life. It may also improve the quality of delivered care when using the data per hospital type as mirror information for daily practice.

Therefore, the objective of our study was to investigate the PFS duration of $A B C$ patients treated with exemestane plus everolimus in the Southeast of the Netherlands shortly after registration of everolimus (i.e. the period 2012-2014) and, foremost, whether implementation and PFS was associated with hospital type. 


\section{Material and methods}

\section{SOutheast Netherlands Advanced BREast cancer (SONABRE) registry}

Data for this study were obtained from the SONABRE registry of the Southeast Netherlands Breast Cancer Consortium (NCT03577197), which is currently running in seven hospitals and shortly extending to another seven hospitals in the Netherlands. In line with Dutch practice, the hospitals were classified as academic, teaching, or non-teaching hospitals. The treatment of patients with $A B C$ in the Netherlands is not centralised and thus performed at all hospitals. The SONABRE registry is an ongoing observational cohort study aimed at the inclusion of all patients diagnosed with $A B C$ since 2007, irrespective of received treatment and irrespective of date of primary breast cancer diagnosis. Specially trained registration clerks collect the data retrospectively from medical files. The data concerns patient, treatment, and tumour characteristics, hospitalisation, and outcomes (progression and death). The SONABRE registry has already been effectively used to perform real-life studies on safety and effectiveness of various treatments used in $A B C$ patients ${ }^{13,14}$. The Medical Research Ethics Committee of Maastricht University Medical Centre approved the registry (no. 15-4-239).

\section{Study population}

The European Medicines Agency (EMA) approved exemestane plus everolimus on 31 July 2012. We selected patients from the SONABRE registry when treatment was started before 31 December 2014, which we had defined as the implementation phase' (Supplementary Figure S3.1.1). Data lock was on July $25^{\text {th }}, 2017$.

\section{Endpoints and statistical analyses}

The endpoint of the study was the PFS of $A B C$ patients treated with exemestane plus everolimus in the implementation phase and, foremost, per hospital type (academic, teaching and non-teaching). PFS was defined as the interval between start of treatment with exemestane plus everolimus and the earliest date of progression or death from any cause, whichever came first. Dates for censoring included last update in case of ongoing treatment, date of next treatment in case of switching to a different therapy without progression, or last date of exemestane plus everolimus treatment in case of no further treatment and no information on progression. PFS was estimated using the Kaplan-Meier (KM) method and compared between hospital types using the log-rank test. Based on the observation that the number of PFS-events mainly differed between hospital types in the first 12-week treatment period, we additionally performed a post-hoc 12-week conditional analysis for patients who used exemestane plus everolimus treatment for longer than 12 weeks. The multivariable Cox proportional 
hazards model was performed by type of hospital and adjusted for potential confounding factors at start of exemestane plus everolimus treatment. Factors considered in the model were age ( $<65$ versus $\geq 65$ years), metastatic-free interval (de novo, 3-24 months versus $>24$ months), visceral disease (yes versus no), number of metastatic sites $(1,2$ versus $\geq 3$ ), prior (neo)adjuvant endocrine therapy (yes versus no), prior (neo)adjuvant chemotherapy (yes versus no), number of previous systemic therapies (1, 2 versus $\geq 3$ ), and start dose everolimus ( $<10 \mathrm{mg}$ versus $\geq 10 \mathrm{mg}$ ). Performance status was not included in the model, because of the high number of missing items and also considered to be related to the other included factors. Factors with a p-value below 0.25 in the univariable model were included in the multivariable model.

To evaluate the implementation of exemestane plus everolimus treatment, we additionally assessed treatment duration for exemestane -irrespective of everolimus-, exemestane plus everolimus treatment duration, and for exemestane plus everolimus dosed at $10 \mathrm{mg}$ using the KM method and log-rank tests. Further, we assessed the reasons for treatment discontinuation (i.e. progression, toxicity or other reasons).

Baseline characteristics of the patients between hospital types were compared using Pearson's chi-squared tests for categorical variables and the nonparametric Kruskal-Wallis test for median age. Overall follow-up time was estimated using the reverse KM method.

\section{Results}

\section{Study population}

At time of data lock, we registered 244 patients who were treated for HR-positive/HER2-negative ABC in 2012-2014 in the SONABRE registry, of whom 65, 123 , and 56 patients were treated in the academic ( $n=1$ hospital), teaching $(n=4$ hospitals) and non-teaching hospitals ( $n=2$ hospitals), respectively. A total of 122 patients started treatment with exemestane plus everolimus, of whom 48 patients were treated in an academic, 56 in teaching and 18 in non-teaching hospitals. The first patient started treatment on August $28^{\text {th }}, 2012$ (Supplementary Figure S3.1.1). The majority of patients were female (99.5\%). The median age was 64 years (range 32-89) (Table 3.1.1). The majority had a metastatic-free interval of $>24$ months (75\%), visceral disease (66\%), and $\geq 3$ lines of previous systemic therapy in the (neo)adjuvant and/or palliative setting (68\%). No statistically significant differences in baseline characteristics were found between hospital types, apart from performance status which was often missing from the patient files. The median follow-up time after start of treatment was 40 months (95\% confidence interval (Cl) 35-45). 
Table 3.1.1 Patient, tumour and treatment characteristics at start of exemestane plus everolimus treatment for advanced breast cancer in 2012-2014.

\begin{tabular}{|c|c|c|c|c|c|}
\hline \multirow[t]{2}{*}{ Characteristic } & \multirow{2}{*}{$\begin{array}{c}\text { Total } \\
\mathrm{N}=122(\%)\end{array}$} & \multicolumn{3}{|c|}{ Type of hospital } & \multirow[t]{2}{*}{$p$-value } \\
\hline & & $\begin{array}{l}\text { Academic } \\
\mathrm{N}=48(\%)\end{array}$ & $\begin{array}{l}\text { Teaching } \\
\mathrm{N}=56(\%)\end{array}$ & $\begin{array}{l}\text { Non-teaching } \\
\mathrm{N}=18(\%)\end{array}$ & \\
\hline \multicolumn{6}{|l|}{ Age } \\
\hline Median (years, range) & $64(32-87)$ & $65(32-87)$ & $63(44-80)$ & $64(50-84)$ & 0.62 \\
\hline \multicolumn{6}{|l|}{ Comorbidities } \\
\hline Any & $61(50)$ & $22(46)$ & $27(48)$ & $12(67)$ & 0.30 \\
\hline Infections & $1(1)$ & $1(2)$ & $0(0)$ & $0(0)$ & 0.46 \\
\hline Respiratory system abnormalities & $8(7)$ & $5(10)$ & $3(5)$ & $0(0)$ & 0.28 \\
\hline Vascular disorders & $43(35)$ & $15(31)$ & $20(36)$ & $8(44)$ & 0.60 \\
\hline Metabolic and nutritional disorders & $10(8)$ & $3(6)$ & $5(9)$ & $2(11)$ & 0.79 \\
\hline Performance status & & & & & 0.01 \\
\hline $0-1$ & $51(42)$ & $24(50)$ & $26(46)$ & $1(6)$ & \\
\hline$\geq 2$ & $9(7)$ & $4(8)$ & $4(7)$ & $1(6)$ & \\
\hline Unknown & $62(51)$ & $20(42)$ & $26(46)$ & $16(89)$ & \\
\hline Metastatic-free interval & & & & & 0.54 \\
\hline De novo (<3 months) & $26(21)$ & $13(27)$ & $10(18)$ & $3(17)$ & \\
\hline $3-24$ months & $4(3)$ & $1(2)$ & $3(5)$ & $0(0)$ & \\
\hline$>24$ months & $92(75)$ & $34(71)$ & $43(77)$ & $15(83)$ & \\
\hline Visceral disease & $81(66)$ & $29(60)$ & $42(75)$ & $10(56)$ & 0.17 \\
\hline No. of metastatic sites & & & & & 0.63 \\
\hline 1 & $32(26)$ & $13(27)$ & $12(21)$ & $7(39)$ & \\
\hline 2 & $34(28)$ & $12(25)$ & $17(30)$ & $5(28)$ & \\
\hline$\geq 3$ & $56(46)$ & $23(48)$ & $27(48)$ & $6(33)$ & \\
\hline Prior NSAI use & $116(95)$ & $46(96)$ & $53(95)$ & $17(94)$ & 0.95 \\
\hline Prior (neo)adjuvant endocrine therapy & $64(53)$ & $26(54)$ & $27(48)$ & $11(61)$ & 0.60 \\
\hline $\begin{array}{l}\text { Prior lines of endocrine therapy for } \\
A B C \text {, median (range) }\end{array}$ & $2(0-5)$ & $1.5(0-4)$ & $2(0-5)$ & $2(0-4)$ & 0.53 \\
\hline Prior (neo)adjuvant chemotherapy & $48(39)$ & $19(40)$ & $22(39)$ & $7(39)$ & 1.00 \\
\hline $\begin{array}{l}\text { Prior lines of chemotherapy for } A B C \text {, } \\
\text { median (range) }\end{array}$ & $0(0-11)$ & $0(0-4)$ & $0(0-11)$ & $0(0-4)$ & 0.89 \\
\hline No. of previous systemic therapies ${ }^{b}$ & & & & & 0.36 \\
\hline 1 & $14(12)$ & $7(15)$ & $6(11)$ & $1(6)$ & \\
\hline 2 & $25(21)$ & $13(27)$ & $10(18)$ & $2(11)$ & \\
\hline$\geq 3$ & $83(68)$ & $28(58)$ & $40(71)$ & $15(83)$ & \\
\hline
\end{tabular}

ABC: advanced breast cancer; NSAI: non-steroidal aromatase inhibitor. Visceral disease was defined as lung, liver, brain, pleural or peritoneal involvement. Metastatic-free interval was defined as the time between primary breast cancer diagnosis and the detection of distant recurrence. ${ }^{a}$ Data are number (\%) of patients, unless stated otherwise. ${ }^{b}$ Previous systemic therapies include those used in the adjuvant setting or to treat metastatic disease and include chemo- and endocrine therapy.

\section{The effectiveness of exemestane plus everolimus}

The median PFS was 6.3 months $(95 \% \mathrm{Cl} 4.0-8.6)$ for the total population, and for the patients treated in academic, teaching and non-teaching hospitals respectively 8.5 months (95\% Cl 7.7-9.3), 4.2 months (95\% Cl 2.0-6.3), and 5.5 months $(95 \% \mathrm{Cl}$ 4.2-6.7) (log-rank $p=0.20$ ) (Figure 3.1.1). 
In the multivariable analysis, we found that patients treated in teaching hospitals had a borderline significant shorter PFS (adjusted HR 1.5; 95\% Cl 1.0-2.3, p=0.08) and that patients treated in non-teaching hospitals had a comparable PFS (adjusted HR 1.0; $95 \% \mathrm{Cl} 0.5-1.9, \mathrm{p}=0.98$ ) as compared with patients treated in the academic hospital (Table 3.1.2). When looking at the PFS figures, we noted a clear PFS difference in the first 12-week treatment period, for which we next performed a post-hoc 12-week conditional analysis, showing that the difference in PFS between hospital types disappeared (adjusted HR 1.2; 95\% Cl 0.7-2.1, $\mathrm{p}=0.42$, and adjusted HR 1.3; 95\% Cl 0.72.5, $\mathrm{p}=0.49$, respectively) (Table 3.1.2).

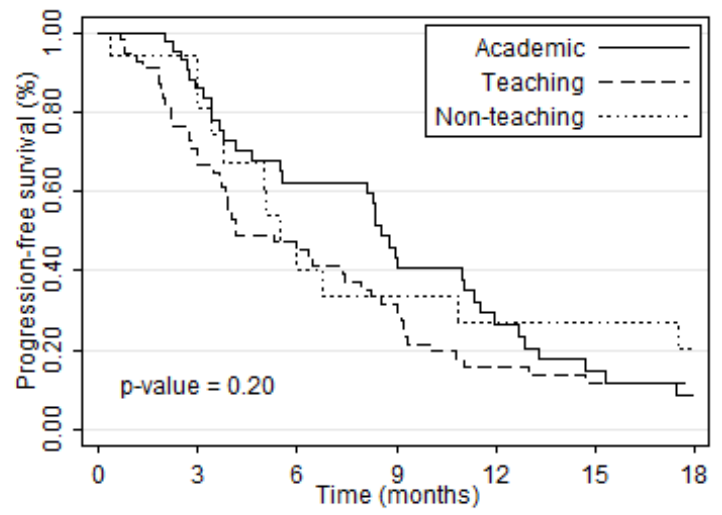

Number at risk

Hospital = academic $\begin{array}{lllllll}48 & 33 & 23 & 16 & 9 & 5 & 3\end{array}$

$\begin{array}{llccccc}\text { Hospital = teaching } 56 & 35 & 24 & 16 & 8 & 6 & 6\end{array}$

Figure 3.1.1 Progression-free survival for exemestane plus everolimus treatment in 2012-2014 by type of hospital (i.e. academic, teaching or non-teaching).

Table 3.1.2 The adjusted hazard ratios for progression-free survival events for exemestane plus everolimus treatment in 2012-2014 performed by type of hospital, from start and after 12-weeks of treatment.

\begin{tabular}{lccccc}
\hline From start of treatment & $\begin{array}{c}\text { Number } \\
(\mathrm{N}=122)\end{array}$ & $\begin{array}{c}\text { Events } \\
(\mathrm{N}=102)\end{array}$ & $\begin{array}{c}\text { Unadj. HR } \\
(95 \% \mathrm{Cl})\end{array}$ & $\begin{array}{c}\text { Adjusted HR } \\
(95 \% \mathrm{Cl})^{\mathrm{a}}\end{array}$ & $\mathrm{p}$-value \\
\hline Academic hospital & 48 & 37 & Reference & Reference & Reference \\
Teaching hospitals & 56 & 51 & $1.4(0.9-2.2)$ & $1.5(1.0-2.3)$ & 0.08 \\
Non-teaching hospitals & 18 & 14 & $1.0(0.6-1.9)$ & $1.0(0.5-1.9)$ & 0.98 \\
\hline After 12-weeks of & Number & Events & Unadj. HR & Adjusted HR & p-value \\
treatment & $(\mathrm{N}=87)$ & $(\mathrm{N}=80)$ & $(95 \% \mathrm{Cl})$ & $(95 \% \mathrm{Cl})^{\mathrm{a}}$ & \\
\hline Academic hospital & 37 & 32 & Reference & Reference & Reference \\
Teaching hospitals & 36 & 35 & $1.2(0.7-1.9)$ & $1.2(0.7-2.1)$ & 0.42 \\
Non-teaching hospitals & 14 & 13 & $1.1(0.6-2.1)$ & $1.3(0.7-2.5)$ & 0.49 \\
\hline
\end{tabular}

$\mathrm{Cl}$ : confidence interval; HR: hazard ratio; unadj: unadjusted. ${ }^{\text {a }}$ Adjusted for potential confounders at start of treatment, i.e. age, metastatic-free interval, visceral disease, number of metastatic sites, prior (neo)adjuvant endocrine therapy, prior (neo)adjuvant chemotherapy, number of previous systemic therapies, start dose everolimus. Factors with a p-value below 0.25 in the univariable model were included in the multivariable model. 
We decided to look more into depth in the patient files as an attempt to find the underlying explanation for the clear difference in PFS in the first 12-week treatment period. Early progression was reported in one out of 48 patients in the academic as compared to nine out of 74 patients in the non-academic hospitals. In the academic hospital, progression was confirmed by imaging. In the non-academic hospitals, early progression was confirmed by imaging in two patients, indicated by a rise in the cancer antigen 15-3 (CA 15-3) in four patients, by symptomatic deterioration in two patients and by unknown reason in one patient.

\section{Delivered everolimus treatment}

Everolimus was started at a $10 \mathrm{mg}$ dose in $81 \%, 84 \%$ and $67 \%$ of the patients treated in the academic, teaching and non-teaching hospitals, respectively (Supplementary Figure S3.1.2A-C).

In the first 12 -week treatment period, $2 \%$ of patients in the academic versus $14 \%$ of patients in teaching and $11 \%$ of patients in non-teaching hospitals had stopped treatment because of progressive disease, whereas respectively $8 \%, 5 \%$, and $11 \%$ of patients had stopped treatment because of toxicity (Supplementary Figure S3.1.2D-F).

There were no significant differences in median treatment durations between hospital types (Supplementary Table S3.1.1).

\section{Discussion}

Here, we present real-life data of patients with HR-positive/HER2-negative ABC, who started treatment with exemestane plus everolimus from August 2012 till December 2014 to assess the implementation and effectiveness of everolimus treatment per hospital type. The real-life median PFS was 6.3 months overall, with a borderline significant difference in PFS between hospital types, due to a difference in number of reported PFS-events in the first 12 weeks of treatment. In the first 12-week treatment period, treatment was discontinued due to early progression in one out of 48 patients in the academic versus nine out of 74 patients in the non-academic hospitals, confirmed by imaging in one and two patients, respectively.

The overall median PFS observed in our real-life study (6.3 months) was slightly shorter as seen in the BOLERO-2 trial ( 7.8 months) ${ }^{6}$. Our real-life population was more heavily pretreated and in a more severe state of disease as compared with the patient population in the BOLERO- 2 trial. For instance, our patients had received more often $\geq 3$ lines of systemic treatment (68\% versus $54 \%)$ and had more often visceral involvement ( $66 \%$ versus $58 \%$ ) than in the BOLERO-2 trial $^{3,6}$. Several phase IIlb studies reported on the efficacy and safety of everolimus, showing that PFS depends on patient selection and everolimus starting dose ${ }^{8-10}$. Although these studies report on real-life 
data, we note that most studies used eligibility criteria and study treatment protocols, and/or included only patients from dedicated cancer centres. In contrast, in our study the patient selection and the decisions on everolimus dosing were fully left to the local oncologist. Knowledge on parameters that are related to treatment outcome is of relevance when informing future patients on the benefits that can be expected, in that way providing a more realistic, individualised prospect.

As far as we know, our real-life study is the first study ever reported assessing everolimus effectiveness per hospital type. We observed that patients treated in teaching hospitals had a borderline significant shorter PFS as compared to those treated in the academic hospital (median 4.2 months versus 8.5 months, adjusted HR 1.5). There seemed to be a small, statistically non-significant, imbalance in number of previous systemic treatment lines between hospital types, but this did not change the HR in the adjusted analysis. By the post-hoc analysis, we showed that the difference in PFS originated from the first 12-week treatment period which seemed to be the result of a different assessment approach. The assessment was not always based on imaging, but also on a rise in CA 15-3. However, caution is needed when interpreting a rise in CA 15-3, especially in the first weeks after treatment initiation, since an initial rise in CA $15-3$ may be followed by a decline with longer treatment duration in $~ 25 \%$ of the patients ${ }^{15,16}$. Therefore, we stress not to use tumour markers as sole criterium for determining progression, especially not in the initial treatment period ${ }^{15}$.

The implementation of exemestane plus everolimus among hospitals types was assessed by determining the number of patients treated with exemestane plus everolimus divided by the number of patients registered and newly diagnosed with HR-positive/HER2-negative $A B C$ in the first 29 months after registration. We had expected to find a similar overall distribution rate of exemestane plus everolimus use among the hospitals types. However, we found that in total only $50 \%$ of patients (122/244 patients) initiated treatment with exemestane plus everolimus, these proportions were $74 \%(48 / 65), 46 \%(56 / 123)$ and $32 \%(18 / 56)$ in the academic, teaching and non-teaching hospitals, respectively. These numbers suggest that the implementation of exemestane plus everolimus was not optimal, and lower in the nonacademic hospitals when compared with the academic hospital. Of note, these proportions need to be interpreted with caution as for some patients exemestane plus everolimus may not be indicated, while for other patients a catch-up may have occurred as exemestane plus everolimus could be provided as third-line treatment and beyond in the first period after approval by the EMA. These two phenomena may have resulted in respectively an under- and overestimation of our results, of which its size is unknown, but expected to be similar between hospital types. An alternative explanation for the lower implementation rate could be that patients diagnosed in nonacademic hospitals had more serious comorbidities. However, as the academic hospital has also a local function (i.e. the only hospital in a large city) and referral for $A B C$ is not frequently done in the region, we do not think this is a major reason for the observed 
differences. The difference in the implementation rate of exemestane plus everolimus by hospital type may also be explained by a difference in experience in prior clinical trials. The implementation may be improved when discussing the best treatment strategies in $A B C$ patients in a team of breast cancer specialists, sharing specific knowledge about the new drug among breast cancer specialists who work at different hospitals.

The present study has a number of strengths. The data were collected by specially trained registration clerks. A thorough data cleaning further contributed to the high quality of the data. Furthermore, we had almost complete exemestane plus everolimus treatment and follow-up data for the patients included due to the long observation period. Last, no exclusion criteria were defined, which makes our study a real-life cohort of patients with $A B C$ treated with exemestane plus everolimus.

Our study also has some limitations which are inherent to the observational design of this study, such as the potential of information bias due to physicians' way of documentation and different assessment schedules. Further, some missing data could not be retrieved. Due to the ongoing nature of our registry, hospitals had different levels of patient inclusion at time of data lock. Patient registration was, however, performed at random, leaving our findings unbiased. Last, we included in total seven hospitals in the implementation phase, which comprised one academic, four teaching and two non-teaching hospitals. The results by type of hospital are therefore indicators for variation between hospitals rather than a comparison between hospital types in the Netherlands and the results should be interpreted accordingly.

In conclusion, in our real-life implementation-phase study, the median PFS was slightly shorter as compared with the BOLERO-2 trial, as is also reported with other treatments and in other tumour types. We observed a difference in the adjusted HR for PFS between hospital types, which was due to a difference in PFS in the first 12-week treatment period. In real-life, the assessment of progressive disease may be more pragmatic and not always based on imaging. As in the first months of treatment the tumour markers may not be a reliable indicator for disease status and toxicity may be more pronounced, we caution to be careful in concluding too early that a treatment is failing. We recommend physicians to broadly share treatment protocols and treatment experience to improve the implementation of therapies. 


\section{References}

1. Cardoso F, Costa A, Senkus E, et al. 3rd ESOESMO international consensus guidelines for Advanced Breast Cancer (ABC 3). Breast. 2017; 31:244-59.

2. Lobbezoo DJ, van Kampen RJ, Voogd AC, et al. Prognosis of metastatic breast cancer subtypes: the hormone receptor/HER2positive subtype is associated with the most favorable outcome. Breast Cancer Res Treat. 2013;141(3):507-14.

3. Baselga J, Campone $M$, Piccart $M$, et al. Everolimus in postmenopausal hormonereceptor-positive advanced breast cancer. N Engl J Med. 2012;366(6):520-9.

4. Cristofanilli M, Turner NC, Bondarenko I, et al. Fulvestrant plus palbociclib versus fulvestrant plus placebo for treatment of hormonereceptor-positive, HER2-negative metastatic breast cancer that progressed on previous endocrine therapy (PALOMA-3): final analysis of the multicentre, double-blind, phase 3 randomised controlled trial. Lancet Oncol. 2016;17(4):425-39.

5. Bachelot T, Bourgier C, Cropet C, et al. Randomized phase II trial of everolimus in combination with tamoxifen in patients with hormone receptor-positive, human epidermal growth factor receptor 2-negative metastatic breast cancer with prior exposure to aromatase inhibitors: a GINECO study. J Clin Oncol. 2012;30(22):2718-24.

6. Yardley DA, Noguchi S, Pritchard $\mathrm{KI}$, et al. Everolimus plus exemestane in postmenopausal patients with $\mathrm{HR}(+)$ breast cancer: BOLERO-2 final progression-free survival analysis. Adv Ther. 2013;30(10):87084.

7. Piccart M, Hortobagyi GN, Campone M, et al. Everolimus plus exemestane for hormonereceptor-positive, human epidermal growth factor receptor-2-negative advanced breast cancer: overall survival results from BOLERO2†. Ann Oncol. 2014;25(12):2357-62.

8. Lousberg L, Jerusalem G. Safety, Efficacy, and Patient Acceptability of Everolimus in the Treatment of Breast Cancer. Breast Cancer (Auckl). 2017;10:239-52.

9. Jerusalem G, Mariani G, Ciruelos EM, et al. Safety of everolimus plus exemestane in patients with hormone-receptor-positive, HER2-negative locally advanced or metastatic breast cancer progressing on prior nonsteroidal aromatase inhibitors: primary results of a phase IIIb, open-label, single-arm, expanded-access multicenter trial (BALLET). Ann Oncol. 2016;27(9):1719-25.

10. Chocteau-Bouju D, Chakiba C, Mignot L, et al. Efficacy and tolerance of everolimus in 123 consecutive advanced ER positive, HER2 negative breast cancer patients. A two center retrospective study. Breast. 2015;24(6):71822.

11. van Steenbergen LN, Rutten HJ, Creemers GJ, et al. Large age and hospital-dependent variation in administration of adjuvant chemotherapy for stage III colon cancer in southern Netherlands. Ann Oncol. 2010;21(6): 1273-8.

12. Spronk PER, van Bommel ACM, Siesling $S$, et al. Variation in use of neoadjuvant chemotherapy in patients with stage III breast cancer: Results of the Dutch national breast cancer audit. Breast. 2017;36:34-8.

13. van Kampen RJW, Ramaekers BLT, Lobbezoo DJA, et al. Real-world and trial-based costeffectiveness analysis of bevacizumab in HER2-negative metastatic breast cancer patients: a study of the Southeast Netherlands Breast Cancer Consortium. Eur J Cancer. 2017; 79:238-46.

14. Seferina SC, Lobbezoo DJ, de Boer M, et al. Real-Life Use and Effectiveness of Adjuvant Trastuzumab in Early Breast Cancer Patients: A Study of the Southeast Netherlands Breast Cancer Consortium. Oncologist. 2015;20(8):856-63.

15. Van Poznak C, Somerfield MR, Bast RC, et al. Use of Biomarkers to Guide Decisions on Systemic Therapy for Women With Metastatic Breast Cancer: American Society of Clinical Oncology Clinical Practice Guideline. J Clin Oncol. 2015;33(24):2695-704.

16. Kim HS, Park YH, Park MJ, et al. Clinical significance of a serum CA15-3 surge and the usefulness of CA15-3 kinetics in monitoring chemotherapy response in patients with metastatic breast cancer. Breast Cancer Res Treat. 2009;118(1):89-97. 


\section{Supplemental material}

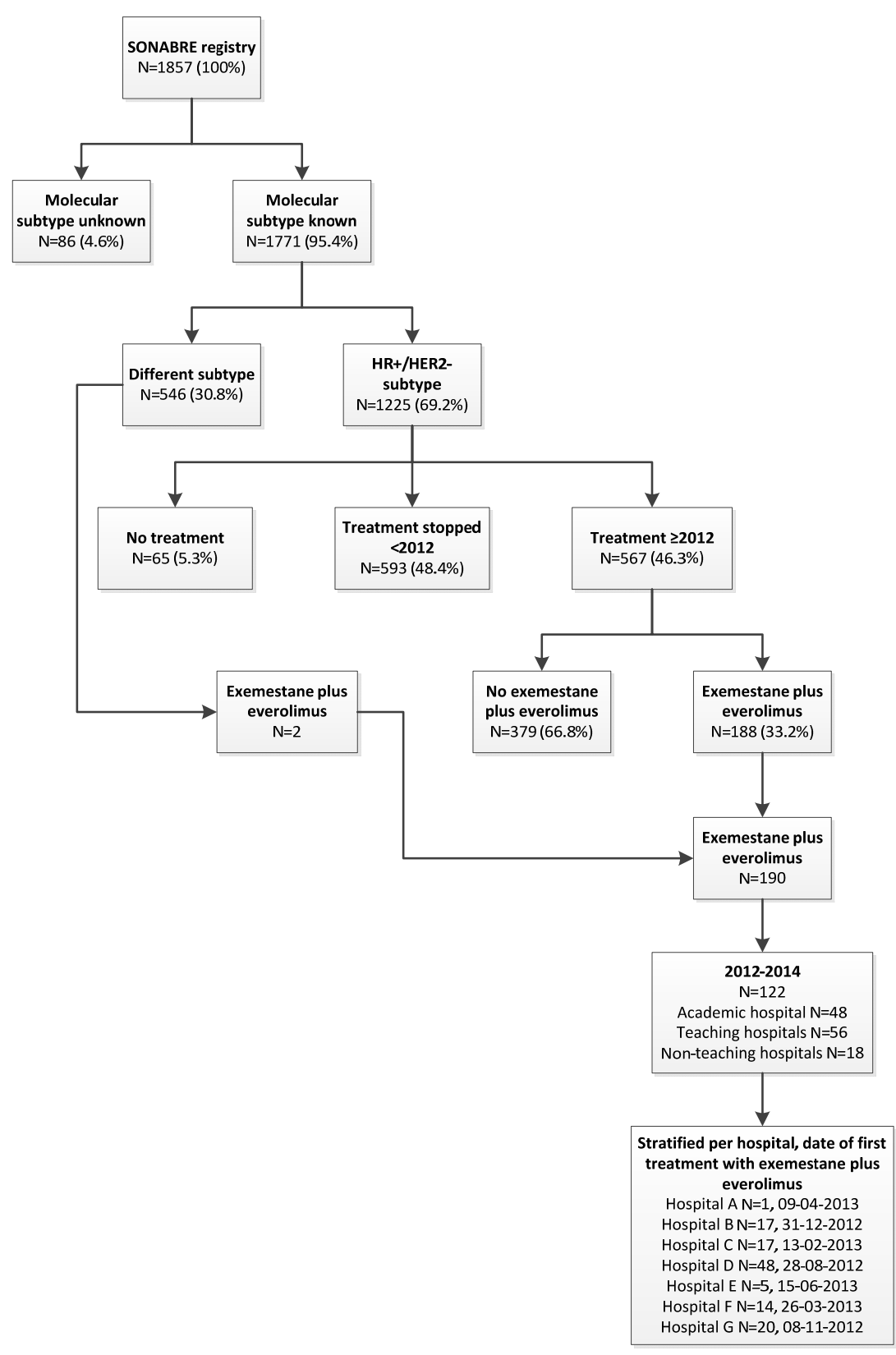

Figure S3.1.1 Study flow chart. 
(A) Academic hospital

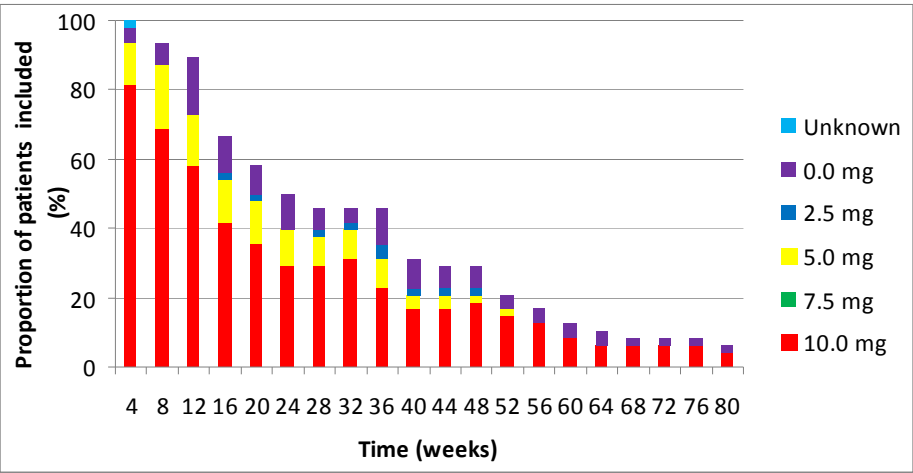

(B) Teaching hospitals

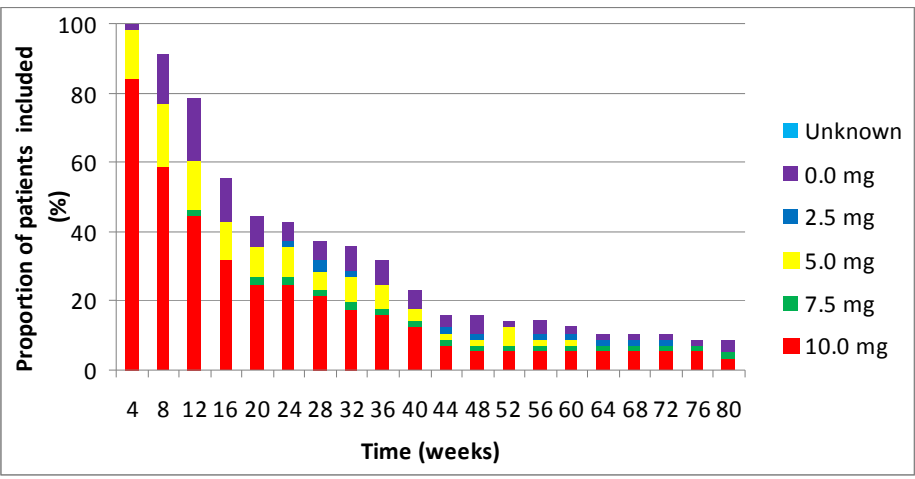

(C) Non-teaching hospitals

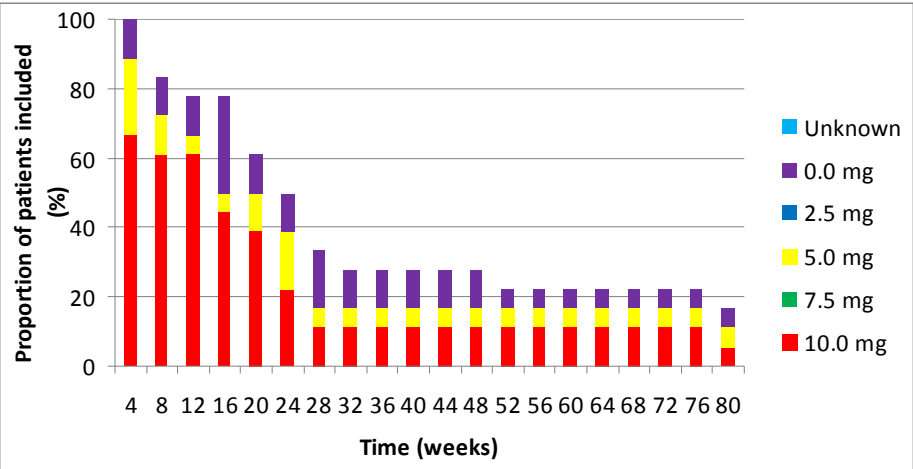


(D) Academic hospital

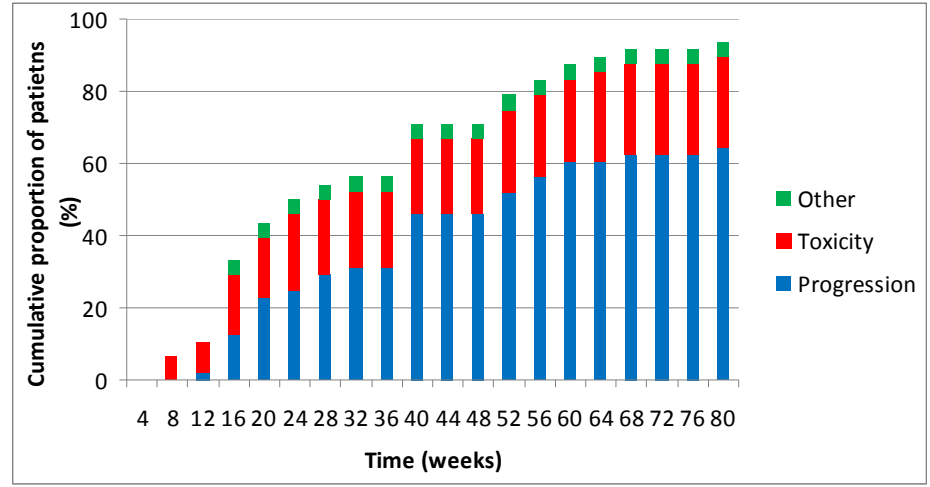

(E) Teaching hospitals

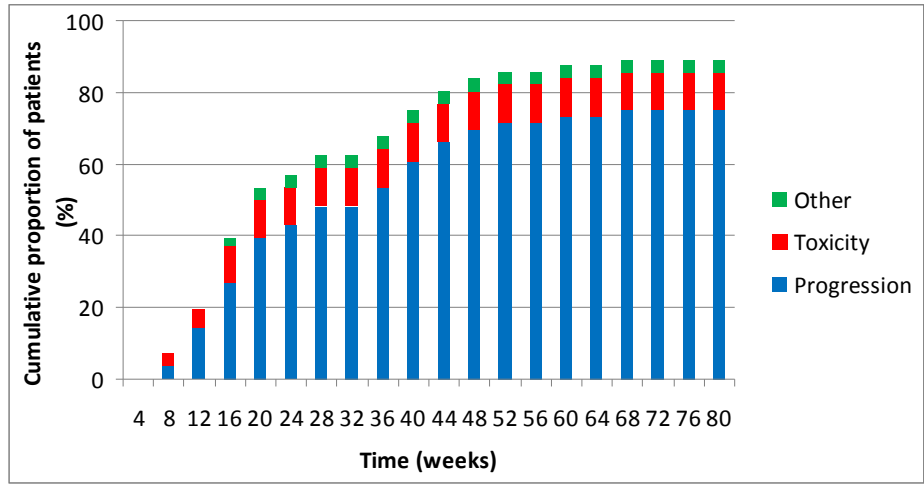

(F) Non-teaching hospitals

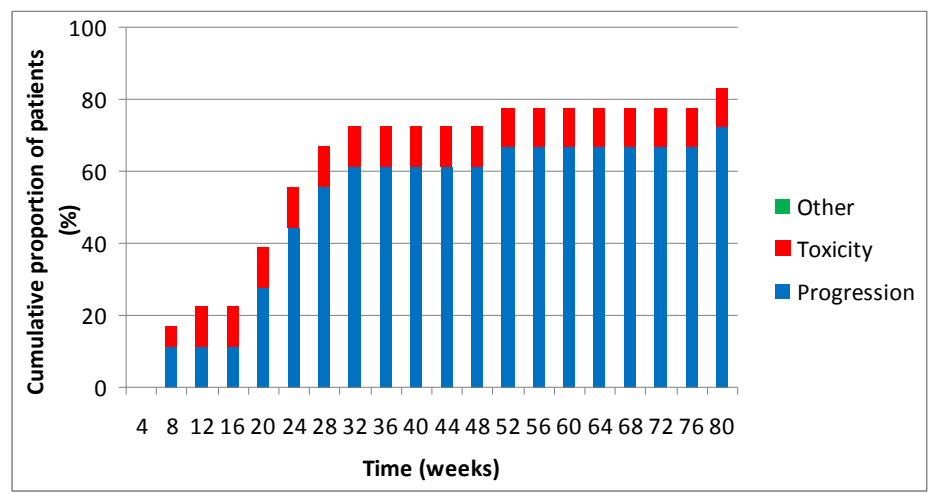

Figure S3.1.2 Actual delivered everolimus dose in time during exemestane plus everolimus treatment in 2012-2014 performed by type of hospital (A, B, C). Reasons for permanent discontinuation of exemestane plus everolimus in time in 2012-2014 performed by type of hospital (D, E, F). 
Implementation and effectiveness of exemestane plus everolimus per hospital type

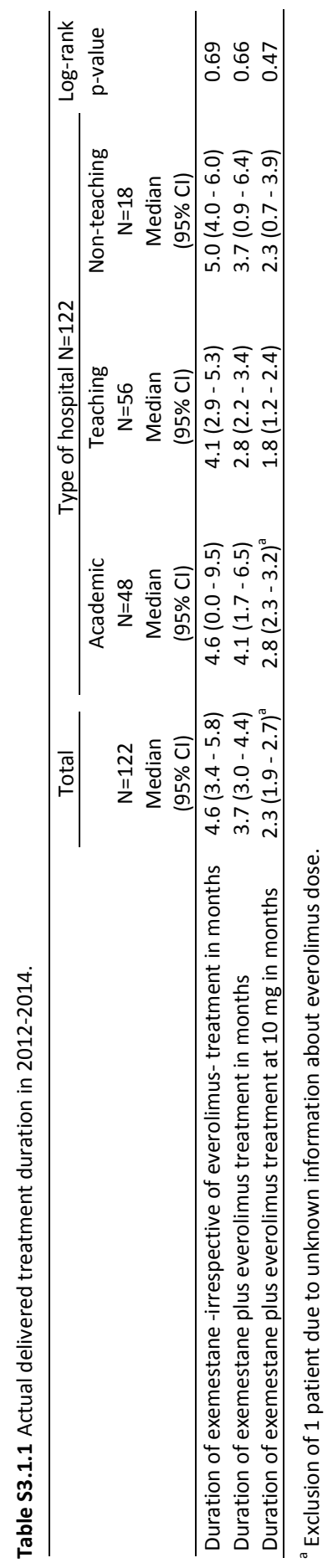






\section{Chapter}

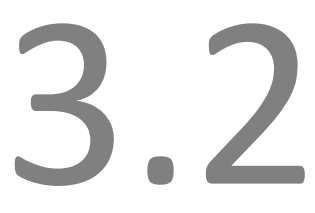

The impact of dose and simultaneous use of acid-reducing agents on the effectiveness of vemurafenib in metastatic BRAF V600 mutated melanoma: a retrospective cohort study

L.M. Knapen, R.H.T. Koornstra, J.H.M. Driessen, B. van Vlijmen, S. Croes, S. Schalkwijk, A. Colbers, W.R. Gerritsen, D.M. Burger, F. de Vries, N.P. van Erp Target Oncol. 2018;13(3):363-370 


\section{Abstract}

\section{Background}

The impact of dose and simultaneous use of acid-reducing agents (ARAs) on the effectiveness of vemurafenib is unknown.

\section{Objectives}

To determine the association between progression of metastatic BRAF V600 mutated melanoma and (1) dose reductions of vemurafenib, and (2) simultaneous use of vemurafenib and ARAs.

\section{Patient and methods}

A retrospective cohort study of 112 first-line vemurafenib users for melanoma was conducted (March 2012-March 2016), using electronic patient records and pharmacy dispensing records of a Dutch academic hospital. Cox regression analysis was used to estimate risk of progression with full-dose $(n=64)$ versus reduced-dose vemurafenib $(n=48)$ and with simultaneous use of vemurafenib and ARAs $(n=35)$ versus vemurafenib alone $(n=77)$. Analyses were adjusted for age and sex.

\section{Results}

In total, disease progression occurred in $55 \%$ of treated patients on vemurafenib, with a median progression-free survival of 6.0 (95\% confidence interval (Cl) 5.0-6.9) months. Compared to patients on vemurafenib alone, there was no increased risk of progression among patients requiring vemurafenib at a reduced dose or among patients receiving simultaneous therapy with vemurafenib and ARAs. In addition, there was no increased risk of progression among patients who used reduced-dose vemurafenib and ARAs versus those receiving full-dose vemurafenib as sole therapy. However, a tendency for progression was observed among patients who used full-dose vemurafenib and ARAs versus full-dose vemurafenib alone (adjusted hazard ratio (HRa) $2.37 ; 95 \% \mathrm{Cl} 0.97-5.76$ ), which became statistically significant in a sensitivity analysis (HRa 4.56; 95\% Cl 1.51-13.75).

\section{Conclusions}

There was no association between the use of vemurafenib in a reduced dose or the simultaneous use of vemurafenib and ARAs and the risk of progression. In addition, there was no association between the simultaneous use of vemurafenib in a reduced dose and ARAs and the risk of progression. However, patients tolerating full-dose vemurafenib simultaneously with ARAs might have an increased risk of progression. This finding requires prospective validation. 


\section{Introduction}

Until recently, the prognosis for melanoma patients with distant metastases was poor, with 1-year survival rates ranging from $33 \%$ to $62 \%$ among patients with stage IV melanoma ${ }^{1}$. Mutations in the BRAF gene can be detected in approximately $40-60 \%$ of metastatic melanoma cases and lead to the constitutive activation of downstream MAP kinase/ERK-signalling, resulting in cell proliferation and survival ${ }^{2}$. Vemurafenib is a potent, selective inhibitor of the mutated BRAF V600 protein kinase ${ }^{2}$. In the BRIM-3 trial, which evaluated vemurafenib as first-line treatment of melanoma with BRAF V600 mutations, progression-free survival (PFS) was significantly longer for patients receiving vemurafenib than for those on dacarbazine, with a median PFS of 6.9 versus 1.6 months, respectively (hazard ratio (HR) 0.38; $95 \%$ confidence interval $(\mathrm{Cl})$ 0.32-0.46; $p<0.001)^{2,3}$. Moreover, this improvement was also reflected in a significantly longer median overall survival (OS) of 13.6 months for vemurafenib as compared to 9.7 months for dacarbazine (HR 0.70; 95\% $\mathrm{Cl} 0.57-0.87 ; \mathrm{p}=0.0008)^{3}$. This trial resulted in the approval of vemurafenib as first-line treatment of metastatic BRAF V600 mutated melanoma by the United States (US) Food and Drug Administration (FDA) in August 2011, followed by the approval by the European Medicines Agency (EMA) in December 2011.

Currently, vemurafenib is not dosed on an individual basis, and no dose increments are allowed. The standard starting dose is $960 \mathrm{mg}$ orally twice daily, which may be reduced in case of toxicity or frailty. After oral intake, the bioavailability of vemurafenib is assumed to be modest ${ }^{4}$. Vemurafenib shows dose-proportional pharmacokinetics from a dose of $240 \mathrm{mg}$ twice daily to $960 \mathrm{mg}$ twice daily ${ }^{5-7}$. Due to the large intra- and interpatient pharmacokinetic variability of vemurafenib, the recommended fixed starting dose will lead to substantial differences in vemurafenib exposure and may thereby result in either under- or overexposure ${ }^{7-9}$. Both are likely to affect treatment outcome, since an exposure-response relationship has been shown for vemurafenib $^{5,9-11}$. Patients tolerating full-dose vemurafenib may be the subgroup underexposed to vemurafenib, while patients experiencing toxicities due to vemurafenib may reflect a subgroup of patients who are overexposed, and thus in need of dose reduction.

Vemurafenib absorption can be decreased with simultaneous use of acid-reducing agents (ARAs), since the solubility of vemurafenib drops with gastric $\mathrm{pH}>2.8^{5,12}$. Data from two American healthcare databases showed that the prevalence of ARA use ranged from 14 to $29 \%$ among melanoma patients ${ }^{12}$. Simultaneous use of proton pump inhibitors (PPIs) was shown to reduce the area under the concentration-time curve of other tyrosine kinase inhibitors by 46 to $61 \%{ }^{13-15}$. Patients using vemurafenib simultaneously with ARAs may therefore be at risk of underexposure to vemurafenib due to the potential interaction between ARAs and vemurafenib ${ }^{12,16}$. 
It is currently unknown whether the use of reduced-dose vemurafenib positively affects treatment outcomes of vemurafenib and whether the use of ARAs negatively affects treatment outcomes of vemurafenib. The objectives of this study were, therefore, to determine the association between the progression of metastatic BRAF V600 mutated melanoma and (1) dose reductions of vemurafenib, and (2) simultaneous use of ARAs.

\section{Materials and methods}

\section{Data source}

The treatment of patients with metastatic melanoma in the Netherlands is centralised in 14 designated medical centres. Requirements of the national reimbursement system are such that vemurafenib prescriptions can only be dispensed at the pharmacy that is affiliated to the respective melanoma medical centre, which ensures a perfect capture of vemurafenib exposure. Data for this study were obtained from both the pharmacy dispensing data and the electronic patient records of an academic hospital in the Netherlands, which were linked at an individual patient level. The pharmacy dispensing data included information on (co-)medication, such as ARAs, cobimetinib, potent inductors or inhibitors of cytochrome P450 enzyme (CYP3A4) and P-glycoprotein (P-gp), the prescribed dose of vemurafenib, and the starting date of vemurafenib treatment. The electronic patient records contained information on the date and reason for vemurafenib discontinuation (disease progression, toxicity, hospital admission, death, switch to different lines of treatment, and/or surgical interventions). All data entries were checked by independent researchers.

\section{Study population}

With the approval of the Research Ethics Board (Arnhem/Nijmegen), we conducted a retrospective cohort study and reviewed pharmacy dispensing data to identify patients who had started vemurafenib as first-line treatment for metastatic BRAF V600 mutated melanoma between 17 March 2012 and 17 March 2016. The first vemurafenib dispensing by the pharmacy was defined as the start of follow-up (index date). A total of 115 patients were selected based on the pharmacy dispensing data, and we included all patients aged $18+$ years at the start of follow-up who had at least one vemurafenib prescription dispensed. Two patients were excluded because their melanoma was not BRAF V600 mutated after revision by our molecular biology laboratory. One patient was excluded because the electronic patient record was lacking information on the safety and effectiveness of vemurafenib (Figure 3.2.1). No other exclusion criteria were defined in order to reflect a 'real-life' retrospective cohort. 


\section{Exposure}

The follow-up was divided into 7-day intervals. Patients were followed up to death, censoring, or disease progression (the outcome of interest) as recorded in the electronic patient files, whichever came first. Reasons for censoring included switching to different lines of therapy before disease progression occurred, toxicity, surgical interventions, loss to follow-up, and the end of the study period.
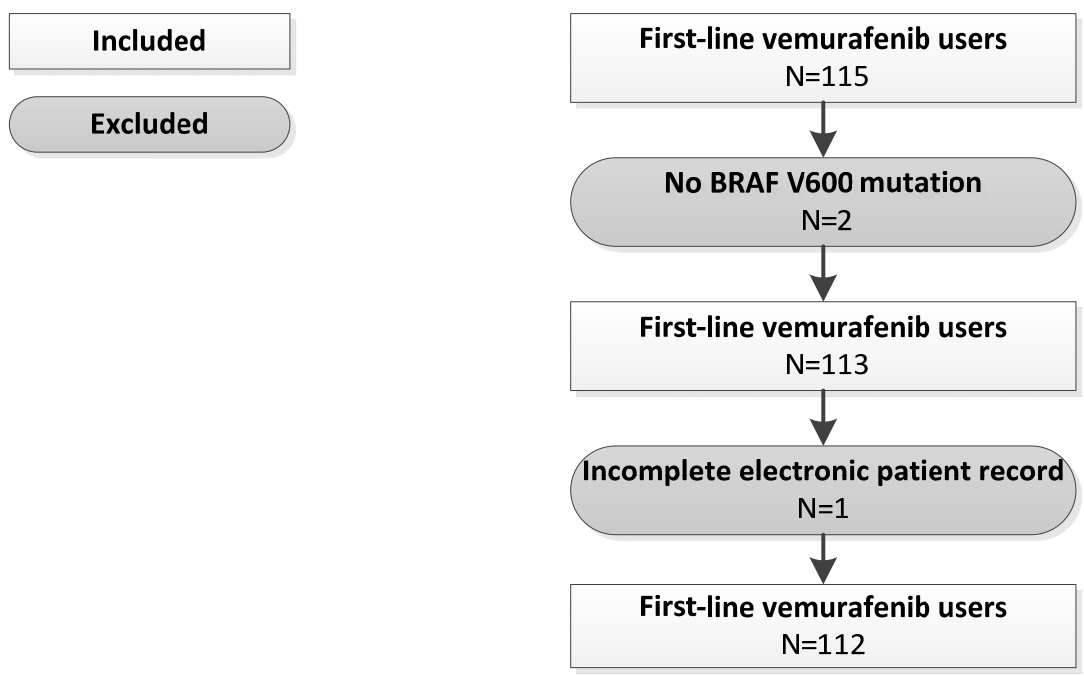

Figure 3.2.1 Flow chart of the study population according to entry criteria.

At the start of each interval, the most recently dispensed dose of vemurafenib was determined using the pharmacy dispensing data. These data were then compared to the dispensed dose of the penultimate vemurafenib dispensed to assess whether a dose change had taken place. Person-time was divided into three categories ('before dose reduction'; 'after dose reduction'; 'no dose reduction') since it was hypothesised that patients experiencing toxicities while using full-dose vemurafenib could be overexposed and therefore in need of a dose reduction. When a dose reduction did occur, time was separated into two different exposure categories: 'before dose reduction' and 'after dose reduction'. Patients using full-dose vemurafenib during their entire treatment were classified as 'no dose reduction' (960 mg twice daily).

Furthermore, prior to the start of each interval, exposure to ARAs (PPIs, $\mathrm{H} 2$ receptor antagonists, or antacids) was determined using the pharmacy dispensing data. ARAs were identified by the following Anatomical Therapeutic Chemical (ATC) codes: A02BC, $A 02 B A$, and $A 02 A^{17}$. A current ARA user was defined as someone who had his most 
recent ARA dispensed within the 30 days prior to the start of an interval, while past ARA users had received their most recent dispensing $>30$ days previously.

\section{Outcomes}

The outcome of interest was PFS in patients with metastatic BRAF V600 mutated melanoma on first-line vemurafenib. Progression was either assessed radiologically or clinically by the treating physician. PFS was defined as the time from dispensing of first vemurafenib prescription until the occurrence of new lesions or the progression of existing lesions.

\section{Potential confounders}

Age and sex were considered to be potential confounders and were incorporated as covariates into the analyses. Sex was determined at baseline, and age was determined time dependently.

\section{Co-medication}

The influence of concomitant administration of potent inductors or inhibitors of CYP3A4 and P-gp was assessed because vemurafenib is mainly metabolised by CYP3A4 and is a substrate of P-gp.

\section{Statistical analysis}

Regression analysis with the Cox proportional hazards models (PHREG procedure, SAS version 9.4; SAS Institute, Cary, NC, USA) was used to estimate disease progression among (1) full-dose vemurafenib users $(n=64)$ versus reduced-dose vemurafenib users $(n=48)$; (2) simultaneous ARA use $(n=35)$ versus no ARA use $(n=77)$; and (3) the combination of both exposure groups. Multivariate analysis was used to adjust for age and sex. PFS was estimated using the Kaplan-Meier method.

\section{Sensitivity analysis}

A sensitivity analysis was performed using only pharmacy dispensing data to determine the discontinuation date of vemurafenib exposure. This discontinuation date was added to the previously defined censoring events. Discontinuation of vemurafenib was defined as the estimated end date of the dispensing based on the total number of dispensed tablets and the number of prescribed tablets per day. In the case of no new dispensing during the 28 days after the estimated end data, patients were censored at the estimated end date of the dispensing. This maximum tolerated gap period of vemurafenib was similar to the maximum discontinuation period in clinical trials. In another sensitivity analysis, the time-window of the time since the most recent ARA 
prescription (i.e. current use) was varied to 15 or 60 days, respectively, prior to the start of an interval. ARAs are likely to be dispensed for either a short period of time or for a long period of time, but they may also be used by patients on an 'as needed basis'.

\section{Results}

\section{Study population at baseline}

Among the 112 patients fulfilling inclusion criteria (Figure 3.2.1), 45 were female (40.2\%) and the median age at index date was 60 (range 26-81) years. In total, 64 patients were using full-dose vemurafenib during the entire study period, and 35 patients were using ARAs. The ARAs used by the patients in this study were the PPIs esomeprazole, omeprazole, and pantoprazole, the H2-receptor antagonist ranitidine, and the antacids magnesium hydroxide and ordinary salt combinations. Further, four patients were treated with vemurafenib in combination with cobimetinib. Of those four patients, two were using full-dose vemurafenib during the entire study period and two were using ARAs. None of the patients treated with vemurafenib concomitantly used strong CYP3A4 or P-gp inducers or inhibitors.

\section{Study population at outcome}

The patient outcome characteristics are shown in Table 3.2.1. In total, 62 patients (55.4\%) showed disease progression on first-line vemurafenib. Censoring occurred in 50 patients $(44.6 \%)$, mainly due to switching to immunotherapy before disease progression occurred $(20.5 \%)$, toxicity $(12.5 \%)$, surgical resection $(3.6 \%)$, or loss to follow-up (2.7\%). Six patients (5.4\%) were still on vemurafenib at the end of the follow-up period. The median PFS was 6.0 (95\% Cl 5.0-6.9) months.

Table 3.2.1 Outcome characteristics of metastatic BRAF V600 mutated melanoma patients ( $\mathrm{N}=112)$

\begin{tabular}{lc}
\hline Outcome characteristics & $\mathrm{N}(\%)$ \\
\hline Progression & $62(55.4)$ \\
Female & $28(45.2)$ \\
Male & $34(54.8)$ \\
Censoring & $50(44.6)$ \\
Switch to different lines of therapy & $23(20.5)$ \\
Toxicity & $14(12.5)$ \\
End of follow-up period & $6(5.4)$ \\
Surgical intervention & $4(3.6)$ \\
Lost to follow-up & $3(2.7)$ \\
Death & $0(0)$ \\
\hline
\end{tabular}




\section{Dose reductions of vemurafenib and/or simultaneous use of ARAs and risk of disease progression}

Patients using vemurafenib in a reduced dose had no increased risk of disease progression as compared to full-dose vemurafenib users (adjusted HR (HRa) 1.12; 95\% Cl 0.64-1.61) (Table 3.2.2). Vemurafenib users who had used ARAs within the past 30 days had no increased risk of disease progression (HRa 1.23; 95\% $\mathrm{Cl} 0.53-2.85$ ) as compared to vemurafenib users not using ARAs. There was no increased risk of progression among patients who used reduced-dose vemurafenib and ARAs versus those on full-dose vemurafenib alone (HRa 1.00; $95 \% \mathrm{Cl} 0.45-2.20$ ). However, a tendency for disease progression was observed among patients who had used full-dose vemurafenib and ARAs at the same time versus those who used full-dose vemurafenib alone (HRa 2.37; 95\% Cl 0.97-5.76).

Table 3.2.2 Dose reductions of vemurafenib and/or simultaneous use of acid-reducing agents and risk of disease progression of metastatic BRAF V600 mutated melanoma.

\begin{tabular}{|c|c|c|c|c|}
\hline Vemurafenib exposure & $\begin{array}{l}\text { Events } \\
(\mathrm{N}=62)\end{array}$ & $\begin{array}{c}\text { Incidence rate } \\
\text { (no. of events / } 10 \text { PY) }\end{array}$ & $\begin{array}{l}\text { Unadj. HR } \\
(95 \% \mathrm{Cl})\end{array}$ & $\begin{array}{l}\text { Age-sex adj. HR } \\
(95 \% \mathrm{Cl})\end{array}$ \\
\hline \multicolumn{5}{|l|}{ By dose reduction } \\
\hline No dose reduction (960 mg BID) ${ }^{a}$ & 31 & 18.5 & Reference & Reference \\
\hline Reduced dose $^{\mathrm{b}}$ & 31 & 18.9 & $1.00(0.59-1.67)$ & $1.12(0.64-1.61)$ \\
\hline \multicolumn{5}{|l|}{ By simultaneous ARA use } \\
\hline No ARA exposure & 40 & 14.6 & Reference & Reference \\
\hline Current ARA use $^{c}$ & 9 & 20.4 & $1.24(0.54-2.87)$ & $1.23(0.53-2.85)$ \\
\hline Past ARA use $\mathrm{d}^{\mathrm{d}}$ & 13 & 16.4 & $1.05(0.54-2.03)$ & $1.03(0.53-2.00)$ \\
\hline \multicolumn{5}{|l|}{$\begin{array}{l}\text { By simultaneous ARA use and dose } \\
\text { reductions }\end{array}$} \\
\hline $\begin{array}{l}\text { No dose reduction }(960 \text { mg BID) } \\
\text { no ARA use }\end{array}$ & 19 & 14.6 & Reference & Reference \\
\hline $\begin{array}{l}\text { No dose reduction }(960 \mathrm{mg} \mathrm{BID})^{\mathrm{a}} \text {, } \\
\text { ARA use }\end{array}$ & 12 & 32.5 & $2.49(1.03-6.01)^{*}$ & $2.37(0.97-5.76)$ \\
\hline Reduced dose ${ }^{b}$, no ARA use & 21 & 21.3 & $1.48(0.77-2.85)$ & $1.40(0.72-2.73)$ \\
\hline Reduced dose ${ }^{b}$, ARA use & 10 & 15.2 & $1.02(0.46-2.26)$ & $1.00(0.45-2.20)$ \\
\hline
\end{tabular}

adj: adjusted; ARA: acid-reducing agent; BID: twice daily; $\mathrm{Cl}$ : confidence interval; HR: hazard ratio; no: number; PY: person-years; unadj: unadjusted. ${ }^{a}$ Full-dose (960 mg BID): person-time of patients using $960 \mathrm{mg}$ vemurafenib BID during entire follow-up period; ${ }^{b}$ Reduced dose: person-time of patients using vemurafenib in a reduced dose after the dose reduction; ${ }^{c}$ Current ARA use: most recent ARA prescription within 30 days before start of an interval; ${ }^{d}$ Past ARA use: most recent ARA prescription over 30 days before start of an interval; ${ }^{e}$ Before dose reduction person-time of patients using $960 \mathrm{mg}$ vemurafenib BID not shown due to zero number of events; ${ }^{*}$ Statistically significant at $p<0.05$.

\section{Sensitivity analysis}

In a sensitivity analysis in which we used pharmacy dispensing data to determine the discontinuation date of vemurafenib exposure, only 41 patients showed disease progression (Table 3.2.3). In this sensitivity analysis, there was no association between 
full-dose vemurafenib use versus reduced-dose vemurafenib use and disease progression. In addition, there was no increased risk of progression among patients who used reduced-dose vemurafenib and ARAs versus those on full-dose vemurafenib alone (HRa 0.99; 95\% Cl 0.35-2.75). However, a statistically significant 4.6-fold increased risk of disease progression was found among patients who had used full-dose vemurafenib and ARAs at the same time versus those who had used full-dose vemurafenib alone (HRa 4.56; 95\% Cl 1.51-13.75). In a second sensitivity analysis, current ARA use was determined by varying the time since the most recent ARA prescription to 15 or 60 days, respectively (Table 3.2.4). For both time-windows, we found no associations for current and past ARA use with disease progression as compared to no ARA use.

Table 3.2.3 Sensitivity analysis: dose reductions of vemurafenib and simultaneous use of acid-reducing agents and risk of disease progression of metastatic BRAF V600 mutated melanoma.

\begin{tabular}{|c|c|c|c|c|}
\hline Vemurafenib exposure & $\begin{array}{l}\text { Events } \\
(\mathrm{N}=41)\end{array}$ & $\begin{array}{c}\text { Incidence rate } \\
\text { (no. of events } / 10 \mathrm{PY} \text { ) }\end{array}$ & $\begin{array}{l}\text { Unadj. HR } \\
(95 \% \mathrm{Cl})\end{array}$ & $\begin{array}{l}\text { Age-sex adj. HR } \\
(95 \% \mathrm{Cl})\end{array}$ \\
\hline \multicolumn{5}{|l|}{$\begin{array}{l}\text { By simultaneous ARA use and dose } \\
\text { reductions }{ }^{a}\end{array}$} \\
\hline $\begin{array}{l}\text { No dose reduction }(960 \mathrm{mg} B I D)^{b} \\
\text { no ARA use }\end{array}$ & 14 & 12.7 & Reference & Reference \\
\hline $\begin{array}{l}\text { No dose reduction }(960 \mathrm{mg} \mathrm{BID})^{\mathrm{b}} \text {, } \\
\text { ARA use }\end{array}$ & 10 & 30.8 & $4.83(1.61-14.46)^{*}$ & $4.56(1.51-13.75)^{*}$ \\
\hline Reduced dose $^{c}$, no ARA use & 11 & 15.5 & $1.42(0.60-3.32)$ & $1.22(0.50-2.93)$ \\
\hline Reduced dose ${ }^{c}$, ARA use & 6 & 10.9 & $1.00(0.36-2.75)$ & $0.99(0.35-2.75)$ \\
\hline
\end{tabular}

adj: adjusted; ARA: acid-reducing agent; $\mathrm{Cl}$ : confidence interval; HR: hazard ratio; no: number; PY: personyears; unadj: unadjusted. ${ }^{\text {a }}$ Before dose reduction person-time of patients using $960 \mathrm{mg}$ vemurafenib BID not shown due to zero number of events; ${ }^{b}$ Full-dose (960 mg BID): person-time of patients using $960 \mathrm{mg}$ vemurafenib BID during the entire follow-up period; ${ }^{c}$ Reduced dose: person-time of patients using vemurafenib in a reduced dose after the dose reduction; ${ }^{*}$ Statistically significant at $p<0.05$. 


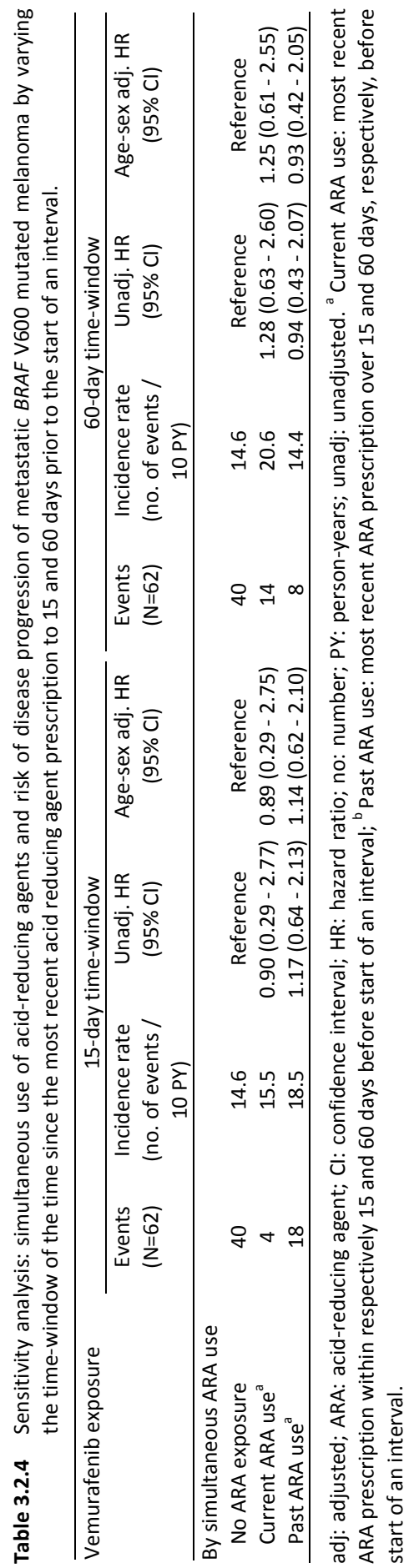




\section{Discussion}

In the present study we found no increased risk of the progression of metastatic BRAF V600 mutated melanoma after dose reductions of vemurafenib, or with simultaneous use of vemurafenib and ARAs, versus vemurafenib alone. In addition, there was no increased risk of progression among patients who used reduced-dose vemurafenib and ARAs versus those who used full-dose vemurafenib alone. However, a tendency for disease progression was observed among patients who used full-dose vemurafenib and ARAs at the same time versus those on full-dose vemurafenib alone when determining exposure to vemurafenib using electronic patient records. The risk for disease progression became significantly elevated to almost fivefold in a sensitivity analysis in which a discontinuation date of vemurafenib (based on pharmacy dispensing records) was added as a censoring event.

To the best of our knowledge, this is the first study to investigate the influence of vemurafenib dose reductions, simultaneous use of vemurafenib and ARA, and full-dose vemurafenib use simultaneously with ARAs on the risk of disease progression in melanoma patients ${ }^{12,16}$. It is presumed that co-treatment with ARAs will reduce vemurafenib plasma concentrations. Vemurafenib has only limited solubility at $\mathrm{pH} 1$ (e.g. the physiologic $\mathrm{pH}$ of the stomach), but has even less solubility at $\mathrm{pH}>2.8$ (i.e. the pKa value of vemurafenib) $)^{5}$. Since the use of ARAs may increase the gastric $\mathrm{pH}$ to $\geq 4$, it is possible that an ARA-vemurafenib interaction occurs that affects vemurafenib uptake $^{5,12}$.

Several previous studies have shown results in line with our findings. Our median PFS was 6.0 (95\% Cl 5.1-6.9) months, which is comparable to the PFS reported in the study of McArthur et al. (6.9 months) and Kramkimel et al. (5.0 months) ${ }^{3,11}$. Furthermore, we found dose reductions in $43 \%$ of the patients using vemurafenib, which is comparable to $38-50 \%$ of the patients requiring a dose reduction in other studies $^{2,9,18}$. In addition, we identified a complete discontinuation of vemurafenib due to toxicity in $13 \%$ of our patients, which is comparable to the $12 \%$ identified in the study by Kramkimel et al. ${ }^{11}$. These results suggest that our population of 'real-life' melanoma patients was comparable to earlier studied melanoma patient populations.

Other studies have reported a relationship between vemurafenib plasma concentrations and treatment outcome (i.e. toxicity and effectiveness), identifying a minimal target steady state concentration of $42 \mu \mathrm{g} / \mathrm{mL}^{9,11}$. High inter- and intrapatient variability at steady-state pharmacokinetics was observed, also when focusing only on the patients taking full-dose vemurafenib ${ }^{9,18}$. In addition, patients treated with a reduced dose of vemurafenib due to adverse events had nearly similar plasma concentrations as patients who tolerated full-dose vemurafenib ${ }^{9}$. These observations indicate that patients requiring dose reductions might be overexposed when taking full-dose vemurafenib ${ }^{9}$. The effect of simultaneous ARA use on treatment outcome will likely be most pronounced in patients with plasma concentrations close to the target 
concentration of $42 \mu \mathrm{g} / \mathrm{mL}$ during the entire treatment period, i.e. the group of patients tolerating full-dose vemurafenib ${ }^{9}$. Therefore, patients tolerating full-dose vemurafenib while simultaneously using ARAs may be of particular risk of early progression due to vemurafenib plasma concentrations below the target level, a possibility which is supported by our results. Modifying the vemurafenib dose based on plasma concentrations could be an elegant and patient friendly way to optimise vemurafenib therapy for individual patients ${ }^{18}$. Unfortunately, data on vemurafenib plasma concentrations were not collected for the patients included in our cohort; such data could be the starting point for future studies.

Information regarding the exposure-response relationship of vemurafenib used in combination with cobimetinib is lacking. The target might be equal or below the earlier identified target for monotherapy with vemurafenib of $42 \mu \mathrm{g} / \mathrm{mL}$, since efficacy was demonstrated with the same dose as used in monotherapy and no interaction between both drugs was identified ${ }^{19}$. It is therefore yet unknown if the increased risk of progression is still present in a population of metastatic BRAF V600 patients who are treated with vemurafenib and cobimetinib and simultaneously using ARAs.

It is important to note the shortcomings of this retrospective database study. True causality cannot be provided with this study. Further, the simultaneous use of vemurafenib with ARAs was difficult to assess. ARAs could be obtained at multiple pharmacies and are available over the counter (without dispensing), making an accurate estimation of co-use challenging. The electronic network linkage of pharmacies allowed us to use drug dispensing data from other healthcare providers; however, completeness of the pharmacy dispensing data could not be guaranteed. This potential misclassification of exposure may have resulted in an underestimation of the effect. Additionally, it is likely that our observed associations are not without residual confounding. Factors that could not be retrieved from the medical records of the patients, and thus were not adjusted for, were food intake (e.g. use of vemurafenib with or without food), the use of food supplements (e.g. grapefruit juice), and herbal supplements (e.g. St. John's wort) ${ }^{4,16,20}$. Lastly, confounding by disease severity might be present. Patients with brain metastases are often treated with the CYP3A4 inductor dexamethasone, which might result in a lower exposure of vemurafenib. We recommend future prospective studies to correct for factors such as food and/or herbal intake, disease severity, and simultaneous use of dexamethasone.

Notwithstanding, our study has a number of important strengths. First of all, we were able to include a relatively large group of metastatic BRAF V600 mutated melanoma patients using vemurafenib as first-line treatment. Moreover, we applied no other exclusion criteria, thereby providing a representative sample of 'real-life' melanoma patients who can therefore be extrapolated to the general population of melanoma patients. Second, we were able to obtain information from both the pharmacy dispensing data and the electronic patient record. The data collected is assumed to be reliable, since all data entries were checked independently by 
researchers. Moreover, due to different data sources we were able to investigate the presence of potential misclassification of vemurafenib exposure. In the sensitivity analyses using only pharmacy dispensing data to determine a discontinuation date of vemurafenib, we found a significant risk of progression in patients using full-dose vemurafenib and ARAs at the same time versus those using full-dose vemurafenib alone. The presence of non-differential misclassification of exposure results in an effect towards the null ${ }^{21}$, which might explain the difference in the results between using the pharmacy data and the electronic patient records data ( $\mathrm{HRa} 4.56 ; 95 \% \mathrm{Cl}$ 1.51-13.75 and HRa 2.37; 95\% $\mathrm{Cl}$ 0.97-5.76, respectively). Third, in order to control for confounding by indication, we only included patients using first-line vemurafenib. It has been found that pretreated patients have an impaired PFS, translating into a trend towards an unfavorable $\mathrm{OS}^{22}$.

In conclusion, there was no association between the use of vemurafenib in a reduced dose or the simultaneous use of vemurafenib and ARAs and the risk of progression. In addition, there was no increased risk of progression among patients who used reduced-dose vemurafenib and ARAs. However, patients using full-dose vemurafenib simultaneously with ARAs might have an increased risk of progression. This finding requires prospective validation. Moreover, future studies are necessary to investigate the added value of measuring vemurafenib concentrations in order to optimise therapy. 
1. Balch CM, Gershenwald JE, Soong SJ, et al. Final version of 2009 AJCC melanoma staging and classification. J Clin Oncol. 2009;27(36): 6199-206.

2. Chapman PB, Hauschild A, Robert C, et al: BRIM-3 Study Group. Improved survival with vemurafenib in melanoma with BRAF V600E mutation. N Engl J Med. 2011;364:2507-16.

3. McArthur GA, Chapman PB, Robert C, et al. Safety and efficacy of vemurafenib in BRAF(V600E) and BRAF(V600K) mutationpositive melanoma (BRIM-3): extended follow-up of a phase 3, randomised, openlabel study. Lancet Oncol. 2014;15(3):323-32.

4. European Medicinces Agency. Zelboraf, Summary of Product Characteristics. 2012. Retrieved from https://www.ema.europa.eu/ documents/product-information/zelborafepar-product-information_en.pdf. Accessed January 2017.

5. US Food \& Drug Administration. Center for Drug Evaluation and Research. Application Number: 2024290rig1s000. Clinical Pharmacology and Biopharmaceutics Review(s). Zelboraf. 2011. Retrieved from https://www.accessdata.fda.gov/drugsatfda_ docs/nda/2011/2024290rig1s000ClinPharmR. pdf. Accessed January 2017.

6. Flaherty KT, Puzanov I, Kim KB, et al. Inhibition of mutated, activated BRAF in metastatic melanoma. N Engl J Med. 2010;363(9):809-19.

7. Grippo JF, Zhang W, Heinzmann D, et al. A Phase I, randomized, open-label study of the multiple-dose pharmacokinetics of vemurafenib in patients with BRAF V600E mutation-positive metastatic melanoma. Cancer Chemother Pharmacol. 2014;73(1): 103-11.

8. Alvarez JC, Funck-Brentano E, Abe E, et al. A LC/MS/MS micro-method for human plasma quantification of vemurafenib. Application to treated melanoma patients. J Pharm Biomed Anal. 2014;97:29-32.

9. Funck-Brentano $E$, Alvarez JC, Longvert $C$, et al. Plasma vemurafenib concentrations in advanced BRAFV $600^{\text {mut }}$ melanoma patients: impact on tumour response and tolerance. Ann Oncol. 2015;26(7):1470-5.

10. Smelick GS, Heffron TP, Chu L, et al. Prevalence of acid-reducing agents (ARA) in cancer populations and ARA drug-drug interaction potential for molecular targeted agents in clinical development. Mol Pharm. 2013;10(11):4055-62.

11. European Medicinces Agency. Sprycel, Summary of Product Characteristics. 2009. Retrieved from https://www.ema.europa.eu/ documents/product-information/sprycelepar-product-information_en.pdf. Accessed January 2017.

12. European Medicinces Agency. Iressa, Summary of Product Characteristics. 2009. Retrieved from https://www.ema.europa.eu/ documents/product-information/iressa-eparproduct-information_en.pdf. Accessed January 2017.

13. European Medicinces Agency. Tarceva, Summary of Product Characteristics. 2009. Retrieved from https://www.ema.europa.eu/ documents/product-information/tarcevaepar-product-information_en.pdf. Accessed January 2017.

14. Willemsen AECAB, Lubbermans FJ, Tol J, et al. Effect of food and acid-reducing agents on the absorption of oral targeted therapies in solid tumors. Drug Discov Today. 2016;21(6):962-76.

15. World Health Organization Collaborating Centre for Drug Statistics Methodology. Retrieved from https://www.whocc.no/atc_ ddd_index/. Accessed January 2017.

16. Kramkimel N, Thomas-Schoemann A, Sakji L, et al. Vemurafenib pharmacokinetics and its correlation with efficacy and safety in outpatients with advanced BRAF-mutated melanoma. Target Oncol. 2016;11(1):59-69.

17. Nijenhuis $C M$, Huitema $A D$, Blank $C$, et al. Clinical Pharmacokinetics of Vemurafenib in BRAF-Mutated Melanoma Patients. J Clin Pharmacol. 2017;57(1):125-8.

18. Ribas A, Zhang W, Chang I, et al. The effects of a high-fat meal on single-dose vemurafenib pharmacokinetics. J Clin Pharmacol. 2014; 54(4):368-74.

19. Rothman KJ, Greenland S and Lash TL (2008). Modern Epidemiology, $3^{\text {rd }}$ edition. Philadelphia, PA: Lippincott, Williams and Wilkins.

20. Ugurel S, Loquai C, Kähler $\mathrm{K}$, et al. A multicenter DeCOG study on predictors of vemurafenib therapy outcome in melanoma: pretreatment impacts survival. Ann Oncol. 2015;26(3):573-82. 
0

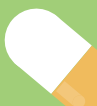

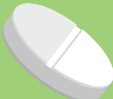

$\varnothing$

0

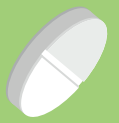




\section{Chapter 4}

Optimisation of treatment in real-life individual

cancer patients 


\section{Chapter 4.1}

Development and validation of an analytical method using UPLC-MS/MS to quantify everolimus in dried blood spots in the oncology setting

L.M. Knapen, Y. de Beer, R.J.M. Brüggemann, L.M. Stolk, F. de Vries, V.C.G. Tjan-Heijnen, N.P. van Erp, S. Croes

J Pharm Biomed Anal. 2018;149:106-113 


\section{Abstract}

\section{Background}

While the therapeutic drug monitoring (TDM) of everolimus has been routinely performed for over 10 years in solid organ transplantation medicine, in order to optimise the balance between effectiveness and toxicity, it is yet uncommon in the treatment of malignancies.

\section{Objectives}

The aim of this study was to develop and validate a bioanalytical method to quantify everolimus in dried blood spots (DBS) to facilitate TDM for the oncology outpatient setting.

\section{Patients and methods}

The hematocrit effect of everolimus was investigated. An $7.5 \mathrm{~mm}$ disk from the central part of the DBS was punched, followed by the extraction of everolimus from the DBS by methanol/acetonitrile (80/20\%) spiked with deuterium-labelled everolimus as internal standard. Subsequently, everolimus was separated and analysed using ultra performance liquid chromatography-tandem mass spectrometry (UPLC-MS/MS). The UPLC-MS/MS method was validated according to the European Medicines Agency (EMA) guideline.

\section{Results}

Everolimus concentrations could be quantified over the range of 3-75 $\mu \mathrm{g} / \mathrm{L}$. The intra- and interassay precision and accuracy of the method were shown to be acceptable (coefficient of variation $\leq 10.7 \%$ and relative error $\leq 4.4 \%$, respectively). The matrix effects appeared to be influenced by the hematocrit effect. The hematocrit effect was tested in a range of $0.20-0.50 \mathrm{~L} / \mathrm{L}$, at which hematocrit accuracy and precision were satisfactory at values $\geq 0.25 \mathrm{~L} / \mathrm{L}$. However, at $0.20 \mathrm{~L} / \mathrm{L}$ hematocrit in combination with high everolimus concentrations of 20 and $40 \mu \mathrm{g} / \mathrm{L}$, the precision was adequate $(\leq 7.4 \%)$, but the accuracy was $>15 \%$ of the nominal concentration. Everolimus was stable in DBS for at least 80 days at $2-8^{\circ} \mathrm{C}$.

\section{Conclusions}

Given these results, the everolimus DBS method has been successfully developed and validated. Special attention is necessary for cancer patients with both a $0.20 \mathrm{~L} / \mathrm{L}$ hematocrit in combination with everolimus concentrations $\geq 20 \mu \mathrm{g} / \mathrm{L}$. A clinical validation for the use of everolimus DBS in cancer patients is currently being undertaken. 


\section{Introduction}

Everolimus is an oral mammalian target of rapamycin (mTOR) inhibitor that inhibits the oncogenic PI3K/AKT/mTOR driver pathway and is approved for the treatment of metastatic (hormone receptor-positive, human epidermal growth factor receptor 2negative) breast cancer, advanced or unresectable neuroendocrine tumours of pancreatic, gastrointestinal or lung origin and metastatic renal cell carcinoma ${ }^{1}$. Despite the proven efficacy, serious adverse events (i.e. NCI-CTCAE grade 3 or higher) occur frequently, being hyperglycaemia (up to $12 \%$ ), stomatitis (up to $9 \%$ ), anemia (up to $9 \%$ ), and diarrhea (up to $6 \%)^{2-5}$. Their management consists of dose reductions, treatment cycle interruptions or even complete discontinuation of everolimus ${ }^{2-5}$.

Currently, everolimus is used at a fixed oral dose of $10 \mathrm{mg}$ once daily, which may be reduced in case of toxicity or fragility ${ }^{1}$. However, it seems important to guide everolimus dosing pharmacokinetically, since everolimus is well known for its large interpatient pharmacokinetic variability, dose proportional pharmacokinetics, relatively small therapeutic window and exposure-response relationship ${ }^{6-11}$. The therapeutic window of area under the concentration-time curve or trough everolimus concentrations is not established yet, although it is assumed to be $\pm 12-26 \mu \mathrm{g} / \mathrm{L}^{8-11}$.

Therapeutic drug monitoring (TDM) is the measurement of drug concentrations in blood to determine pharmacokinetic parameters, in order to optimise individual dosage regimes. Dried blood spot (DBS) sampling by finger prick for the use of TDM has become more common over the years, including the oncology field ${ }^{12-18}$. DBS sampling is minimally invasive and is a promising patient-friendly alternative to venous sampling, since this technique can be performed by patients at their homes and the samples can be sent to the laboratory by regular mail for analysis ${ }^{12,13}$. The physician may benefit from the ease of the DBS sampling method, since analysis results are available before the patient visits the clinic for their (routine) check-up ${ }^{12,13}$. Moreover, it is possible that DBS will be used in the future to determine all routine check-up parameters, enabling the patient to visit the hospital less frequently.

TDM of everolimus, including DBS, is routinely performed in solid organ transplantation medicine for over 10 years to balance between effectiveness and toxicity ${ }^{7,12}$. Therefore, a number of DBS everolimus assays have already been developed and validated ${ }^{12,13,19,20}$. However, these assays cannot easily be extrapolated to the oncology setting, since the upper range of the calibration curves is relatively low (up to a maximum of $50 \mu \mathrm{g} / \mathrm{L}$ ) and the hematocrit effect is often not or only poorly investigated $^{12,13,19,20}$.

It is important to investigate the influence of the hematocrit effect, since it is known that hematocrit varies widely in patients with cancer and that hematocrit can significantly affect analytical results ${ }^{8,13,21,22}$. Lower hematocrit values may impact the disposition of everolimus due to chromatographical effects, causing a higher concentration at the periphery of the DBS as compared to the concentration at the 
centre $^{19,23}$. Moreover, the DBS area may increase at lower hematocrit values due to the reduced blood viscosity and higher permeability through the DBS paper ${ }^{19}$. However, it is important to note that the hematocrit effect is not the only variable influencing the analytical results ${ }^{24}$.

Therefore, the objective of this study was to develop and analytically validate a DBS sampling method for everolimus in the oncology outpatient setting using ultra performance liquid chromatography-tandem mass spectrometry (UPLC-MS/MS). Special attention was paid to the effect of varying hematocrit levels on quantification of everolimus.

\section{Experimental}

\section{Chemicals and reagents}

Everolimus standard vial of $1000 \mathrm{mg} / \mathrm{L}$ in acetonitrile was purchased from Cerilliant Corporation (Round Rock, Texas, USA). As internal standard (IS), deuterium-labelled everolimus (everolimus-D4) was purchased from Toronto Research Chemicals (Toronto, Ontario, Canada). Methanol and formic acid of LC/MS grade were obtained from Biosolve (Valkenswaard, the Netherlands), ammonium acetate and ethanol absolute PA grade were obtained from Merck (Darmstadt, Germany). Ultrapure water was supplied with a Millipore Milli-Q water purification system from Merck-Millipore (Darmstadt, Germany).

A lyophilised certified external quality control of $5.04 \mu \mathrm{g} / \mathrm{L}$ based on human whole-blood (range 3.53-6.56 $\mathrm{\mu g} / \mathrm{L}$ ) from Chromsystems (Gräffelfing, Germany) was used. The sampling paper used was Protein saver 903, ref no. 10531018, and was obtained from Whatman Gmbh (Dassel, Germany). Costar spin-X high performance liquid chromatography (HPLC) $0.2 \mu$ m nylon filter, catalogue no. 8169, was used as the microcentrifuge filter. Everolimus-free blood was collected from healthy volunteers in spray-coated $\mathrm{K}_{2}$ EDTA tubes. Plasma was purchased from Sanquin (Amsterdam, the Netherlands).

\section{Preparation of stock and extraction solutions}

Separate batches of everolimus stock solutions for calibrators and quality control samples were prepared in ethanol $(100 \mathrm{mg} / \mathrm{L})$. The IS stock solution was prepared by dissolving $1 \mathrm{mg}$ of everolimus-D4 in $100.0 \mathrm{~mL}$ methanol (10 mg/L). Both the everolimus and IS stock solution were stored at $2-8^{\circ} \mathrm{C}$. Under these conditions, all solutions are expected to be stable for at least 90 days ${ }^{12,25}$. 
The extraction solution containing IS was prepared by adding $75 \mu \mathrm{L}$ of the IS stock solution $(10 \mathrm{mg} / \mathrm{L})$ to methanol in $25.0 \mathrm{~mL}$. Subsequently, $1.0 \mathrm{~mL}$ of this solution was added to a methanol/acetonitrile $(80 / 20 \%)$ solution resulting in a total $10.0 \mathrm{~mL}$ volume.

\section{Preparation of calibrators and quality control samples}

The everolimus stock solutions for quality controls and calibrators $(100 \mathrm{mg} / \mathrm{L})$ were diluted with millipore water to a concentration of $5 \mathrm{mg} / \mathrm{L}$. These solutions were added to fresh everolimus-free EDTA blood in order to obtain 500 and $50 \mu \mathrm{g} / \mathrm{L}$ working solutions of everolimus in the blood. These solutions were further diluted with fresh everolimus-free EDTA blood to obtain the calibration standards and quality controls (QC). The calibration standards were prepared at the following concentrations 3, 5, 10, 20, 30 and $50 \mu \mathrm{g} / \mathrm{L}$. Additionally, a blank and a zero sample was incorporated in the analysis. In addition, five levels of $Q C$ were prepared: lower limit of quantification (LLOQ) $3 \mu \mathrm{g} / \mathrm{L}$, low-level (QCL) $6 \mu \mathrm{g} / \mathrm{L}$, mid-level (QCM) $25 \mu \mathrm{g} / \mathrm{L}$, high-level (QCH) $40 \mu \mathrm{g} / \mathrm{L}$ and upper limit of quantification (ULOQ) $75 \mu \mathrm{g} / \mathrm{L}$. The LLOQ was set at $3 \mu \mathrm{g} / \mathrm{L}$ which was sufficient for the purpose of the assay in the oncology setting, and adequate sensitivity with minimal signal-to-noise peak area ratio of 5 could be achieved. The prepared whole-blood standards were tested by our validated venous immunosuppressants method (including everolimus), using the certified external QC from Chromsystems, as part of quality assurance before proceeding to sample preparation of DBS according to section 'DBS sample preparation and extraction'.

\section{DBS sample preparation and extraction}

DBS sample preparation and extraction procedure were conducted largely according to methods that have been described previously ${ }^{12}$. In short, the DBS samples were prepared by sampling $30 \mu \mathrm{L}$ of blood with a positive displacement pipette onto sampling paper. The DBS were kept overnight to dry at room temperature and then stored at $2-8^{\circ} \mathrm{C}$ in a sealed plastic bag.

For analysis a $7.5 \mathrm{~mm}$ disk from the central part of the DBS was punched out with an electromagnetic driven hole puncher ${ }^{12}$. The disk was placed into a Costar spin-X HPLC microcentrifuge $0.2 \mu \mathrm{m}$ filter, pressed onto the filter ${ }^{12}$, after which $200 \mu \mathrm{L}$ extraction solution was added. The samples were sonicated for $30 \mathrm{~min}$, centrifuged at $3506 \mathrm{xg}$ for $5 \mathrm{~min}$ and the filter was discarded from the Costar tube. The extracts were vortexed for $5 \mathrm{~s}$ after which they were transferred in a $300 \mu \mathrm{L}$ autosampler polypropylene vial. $6 \mu \mathrm{L}$ was injected onto the UPLC-MS/MS system.

\section{UPLC chromatography and mass-spectrometry}

Liquid chromatography was performed using a Waters UPLC H-class system (Waters, Milford, Massachusetts, USA). The sample was injected directly onto an analytical 
column (Waters Acquity UPLC BEH C18 $1.7 \mu \mathrm{m}$ column, $2.1 \mathrm{~mm} \times 50 \mathrm{~mm}$ ), which was heated to $60^{\circ} \mathrm{C}$. Chromatographic separation was performed by gradient analysis with a flow of $0.5 \mathrm{~mL} / \mathrm{min}$ and a run time of $2.5 \mathrm{~min}$. Mobile phase A consisted of a mixture of $0.1 \%$ formic acid and $2 \mathrm{mmol}$ of ammonium acetate in water, mobile phase $B$ consisted of $0.1 \%$ formic acid and $2 \mathrm{mmol}$ of ammonium acetate in methanol. The following linear gradient was used at the flow rate of $0.5 \mathrm{~mL} / \mathrm{min}$ : time scale (min - min) mobile phase A (\%)/mobile phase B (\%): 0-0.5 35/65 $\rightarrow$ 0.5-2.0 0/100 $\rightarrow$ 2.0-3.5 35/65 (reconditioning of the column). The UPLC system was directly coupled to the mass-spectrometer and the autosampler temperature was held at $10^{\circ} \mathrm{C}$.

Determination of everolimus and the IS (D4-everolimus) was performed on a Xevo TQ-S micro Triple Quadrupole mass-spectrometer fitted with an electrospray ionisation (ESI) source operating in the positive ion mode (Waters, Manchester, UK). The following internal parameters of the device were used: reaction monitoring of $\left(\mathrm{M}+\mathrm{NH}_{4}\right)^{+}$ [precursor/product] $m / z$ transitions everolimus (975.8/908.5) and IS (979.8/912.5), source temperature $150^{\circ} \mathrm{C}$, electrospray capillary voltage $1.0 \mathrm{kV}$, sample cone voltage $50 \mathrm{~V}$, collision energy $16 \mathrm{~V}$, desolvation temperature $400^{\circ} \mathrm{C}$ and dwell time $20 \mathrm{~ms}$ for everolimus and $10 \mathrm{~ms}$ for everolimus-D4. The cone gas (nitrogen) flow was set to $50 \mathrm{~L} / \mathrm{h}$, the desolvation gas (nitrogen) flow to $1000 \mathrm{~L} / \mathrm{h}$, and the collision gas used was argon. The retention time was approximately $1.0 \mathrm{~min}$. System control and data acquisition were performed with MassLynx software (version 4.1, Waters, Etten-Leur, the Netherlands).

\section{Method validation}

The analytical method validation was performed at a standardised $0.40 \mathrm{~L} / \mathrm{L}$ hematocrit value and included selectivity, linearity, precision, accuracy, matrix effects, recovery, carry-over and stability according to international European Medicines Agency (EMA) guideline $^{26}$. It is important to note that currently no regulatory-based DBS validation guideline exists, although it is generally accepted that the validation parameters should include hematocrit effect and blood spot size ${ }^{27}$. Therefore, our analytical validation was extended with the investigation of the influence of these parameters on the precision and accuracy.

Accuracy and precision were calculated by the analysis of the QC DBS samples LLOQ, QCL, QCM, QCH and ULOQ. Each QC DBS sample was freshly prepared in fivefold, processed and measured in three separate analytical runs on 3 different days. The intra-, inter-day and overall precision were calculated. To be specific, overall precision was calculated as the coefficient of variation (CV) based on all 15 measurements over the 3 days. All calculations were performed using Microsoft Office Excel (Version 2010, Microsoft Inc, Radmond, WA). 
To investigate selectivity, DBS samples were made from six everolimus-free volunteers. The selectivity was investigated to assess whether endogenous compounds from DBS could interfere with the detection of everolimus and the IS.

Linearity was investigated by analysing in DBS non-zero whole-blood calibrators in duplicate on three different days. The ratio of everolimus peak area to that of IS area at each concentration was plotted as a function of the everolimus concentration, and were fitted with $1 / x$ weighted linear regression. The linearity was evaluated by means of back-calculated concentrations of the calibrators.

The matrix effect was assessed by spiking six different blank matrices with 5 and $40 \mu \mathrm{g} / \mathrm{L}$ everolimus (concentrations close to the QCL and ULOQ, respectively). The DBS were prepared in duplicate according to Section 'DBS sample preparation and extraction', after which the samples were measured.

We designed an experiment to assess the recovery, and the influence of the hematocrit and stability on the recovery. Whole-blood was prepared at most prevalent hematocrit values of the intended oncology patient population (i.e. 0.35, 0.40 and $0.45 \mathrm{~L} / \mathrm{L}$ ), and spiked with 5 and $40 \mu \mathrm{g} / \mathrm{L}$ everolimus. Subsequently, $15 \mu \mathrm{L}$ of the whole-blood samples were analysed in duplicate. For the recovery, DBS were prepared in duplicate according to Section 'DBS sample preparation and extraction' yet using a blood spotting volume of $15 \mu \mathrm{L}$. The DBS samples were respectively directly analysed, and analysed after being stored for 17 days at $2-8^{\circ} \mathrm{C}$.

Carry-over effects were investigated in fivefold by injecting a blank sample after injecting the ULOQ sample (i.e. $75 \mu \mathrm{g} / \mathrm{L}$ ).

Stability of the DBS samples was investigated during transport conditions in addition to storage at $2-8^{\circ} \mathrm{C}$ or $15-25^{\circ} \mathrm{C}$ pending batch-wise analysis. In order to investigate transport conditions, we performed stability testing after 10 and 17 days, at the temperatures -20 to $-30^{\circ} \mathrm{C}, 2-8^{\circ} \mathrm{C}, 15-25^{\circ} \mathrm{C}$ and $40^{\circ} \mathrm{C}$. In order to investigate the possibility of storing the DBS at $2-8^{\circ} \mathrm{C}$ or $15-25^{\circ} \mathrm{C}$ followed by the batch-wise analysis, we performed stability testing after $10,17,40$ and 80 days. The stability was tested at the 5 and $50 \mu \mathrm{g} / \mathrm{L}$ concentration levels (concentrations close to the QCL and ULOQ, respectively). Moreover, stability was tested over the whole range of the calibration curve to gain insight in the stability over a broad concentration range. Therefore, DBS were prepared in duplicate using whole-blood spiked with 5 and $50 \mu \mathrm{g} / \mathrm{L}$, in addition to all calibrator concentrations (i.e. 3, 5, 10, 20, 30 and $50 \mu \mathrm{g} / \mathrm{L}$ ). Autosampler stability was investigated by storing the extracted everolimus samples in the autosampler for $24 \mathrm{~h}$ with the temperature set at $10^{\circ} \mathrm{C}$.

\section{Influence of hematocrit and concentration on accuracy}

The influence of the hematocrit effect in combination with the everolimus concentration was investigated. DBS were prepared in duplicate using whole-blood prepared at a broad range hematocrit values (i.e. 0.20, 0.25, 0.30, 0.35, 0.40 and 
$0.50 \mathrm{~L} / \mathrm{L}$ ) and spiked with respectively 5,20 and $40 \mu \mathrm{g} / \mathrm{L}$ everolimus ${ }^{8,13,21,22}$. The hematocrit levels used represent the common hematocrit levels of an oncology patient population $^{8,21,22}$.

\section{Influence of hematocrit and spot volume on difference}

The influence of the blood spotting volumes in combination with the hematocrit effect was assessed. DBS were prepared in duplicate using whole-blood prepared at $0.20 \mathrm{~L} / \mathrm{L}$ and $0.40 \mathrm{~L} / \mathrm{L}$, in combination with varying blood spotting volumes of $20-50 \mu \mathrm{L}$ (in $10 \mu \mathrm{L}$ increments) ${ }^{19,23,28}$. The whole-blood was spiked with everolimus concentrations over the whole range of the calibration curve, to gain insight in the hematocrit and blood spotting volume effect over a broad concentration range. The $0.20 \mathrm{~L} / \mathrm{L}$ and $0.40 \mathrm{~L} / \mathrm{L}$ hematocrit levels represent the typically lowest and most prevalent hematocrit levels in the oncology population ${ }^{8,21,22}$.

\section{Results and discussion}

\section{Validation procedure}

The method fulfilled all acceptance criteria regarding accuracy and precision of the calibrators and QCs. The acceptance criteria regarding the calibrators was a maximum accuracy of $\leq 15 \%$ as compared to the nominal concentration, except for $\leq 20 \%$ for the LLOQ, and regarding the QCS a maximum intra- and inter-day precision of $\leq 15 \%{ }^{26}$. The deviation of the individual calibration standards was respectively highest at $3 \mu \mathrm{g} / \mathrm{L}$ (17.1\%) and lowest at $50 \mathrm{\mu g} / \mathrm{L}$ (2.7\%) (results not provided). Table 4.1.1 shows the validation results regarding intra- and inter-day accuracy and precision of the QCs. The highest CV value for precision and percentage for accuracy for the QCs was respectively $\leq 10.7 \%$ and $\leq 7.6 \%$ of the nominal value ${ }^{26}$. A chromatogram of a blank and a blank with the addition of the $I S$ is shown in Figure 4.1.1A and $B$, respectively, and $a$ chromatogram of the LLOQ and QCM is shown in Figure 4.1.2A and $B$, respectively. The peak of everolimus at the LLOQ had a signal-to-noise ratio $(\mathrm{S} / \mathrm{N})$ of 13 for everolimus, and 36 for the IS. The limit of detection was set at three times the noise, and was calculated to be $0.6 \mu \mathrm{g} / \mathrm{L}$. 
Table 4.1.1 Accuracy and precision results of the quality controls for everolimus dried blood spot.

\begin{tabular}{|c|c|c|c|c|c|}
\hline $\begin{array}{l}\text { Nominal } \\
\text { concentration } \\
(\mu \mathrm{g} / \mathrm{L}) \\
\end{array}$ & $\begin{array}{c}\text { Intra-day } \\
\text { accuracy }(\mathrm{N}=5) \\
(\%)\end{array}$ & $\begin{array}{c}\text { Inter-day } \\
\text { accuracy }(\mathrm{N}=15) \\
(\%)\end{array}$ & $\begin{array}{c}\text { Intra-day } \\
\text { precision }^{\text {a }}(\mathrm{N}=5) \\
(\mathrm{CV} \%)\end{array}$ & $\begin{array}{c}\text { Inter-day } \\
\text { precision }^{\mathrm{b}}(\mathrm{N}=15) \\
(\mathrm{CV} \%)\end{array}$ & $\begin{array}{l}\text { Overall precision } \\
\qquad \begin{array}{c}(\mathrm{N}=15) \\
(\mathrm{CV} \%)\end{array} \\
\end{array}$ \\
\hline LLOQ (3) & 107.6 & 104.4 & 10.7 & 0.0 & 8.5 \\
\hline $\mathrm{QCL}(6)$ & 106.4 & 103.0 & 6.7 & 1.9 & 5.3 \\
\hline QCM (25) & 95.6 & 100.6 & 9.9 & 2.8 & 7.8 \\
\hline $\mathrm{QCH}(40)$ & 103.6 & 100.7 & 3.6 & 0.6 & 5.8 \\
\hline ULOQ (75) & 98.1 & 98.9 & 8.0 & 0.0 & 6.4 \\
\hline
\end{tabular}

$\mathrm{CV}(\%)$ : coefficient of variation; LLOQ: lower limit of quantification; $\mathrm{QCH}$ : quality control high-level; $\mathrm{QCL}$ : quality control low-level; QCM: quality control mid-level; ULOQ: upper limit of quantification. ${ }^{a}$ Others may refer to this as 'within-day precision'. ' ${ }^{\text {O }}$ Others may refer to this as 'between-day precision'. Overall precision at each concentration was calculated as the CV\% based on all 15 measurements over the 3 days. Data of the worst-case accuracy $(n=15)$ and intra-day precision $(n=5)$ were reported.

\section{(A)}
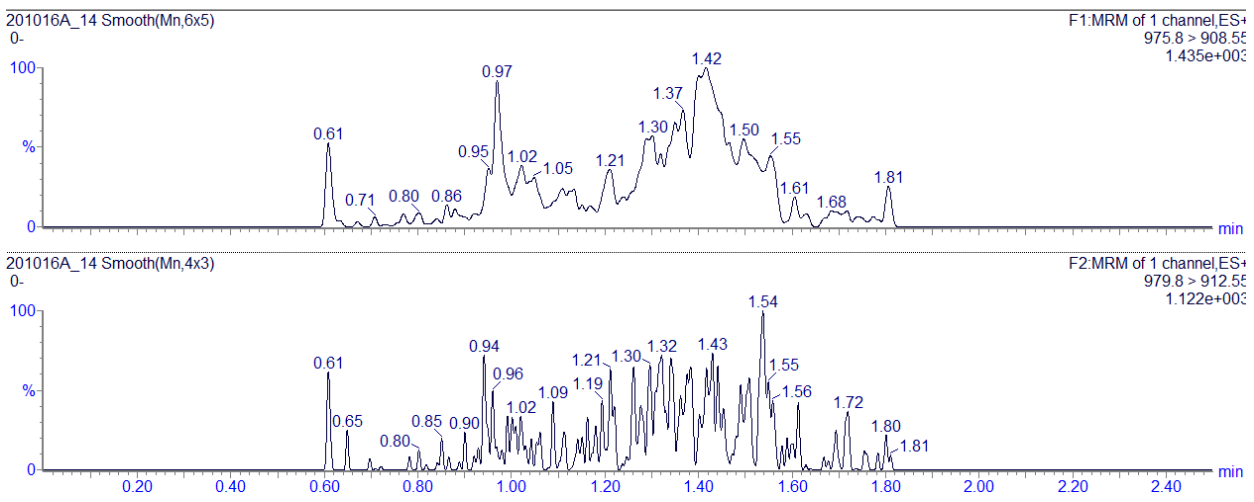

\section{(B)}

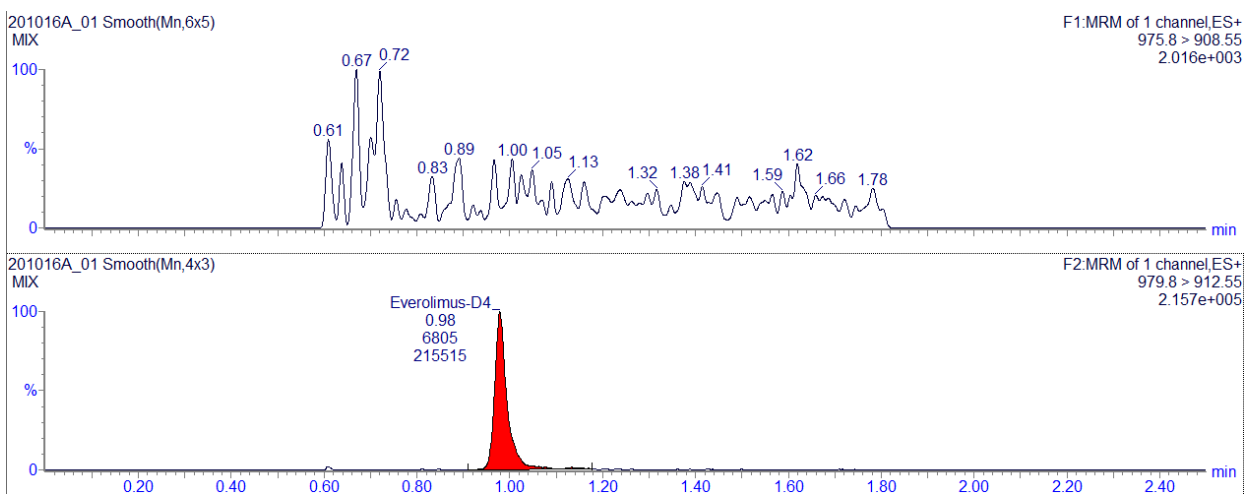

Figure 4.1.1 A chromatogram of a blank (A), and a blank with the addition of the internal standard (B). 
(A)

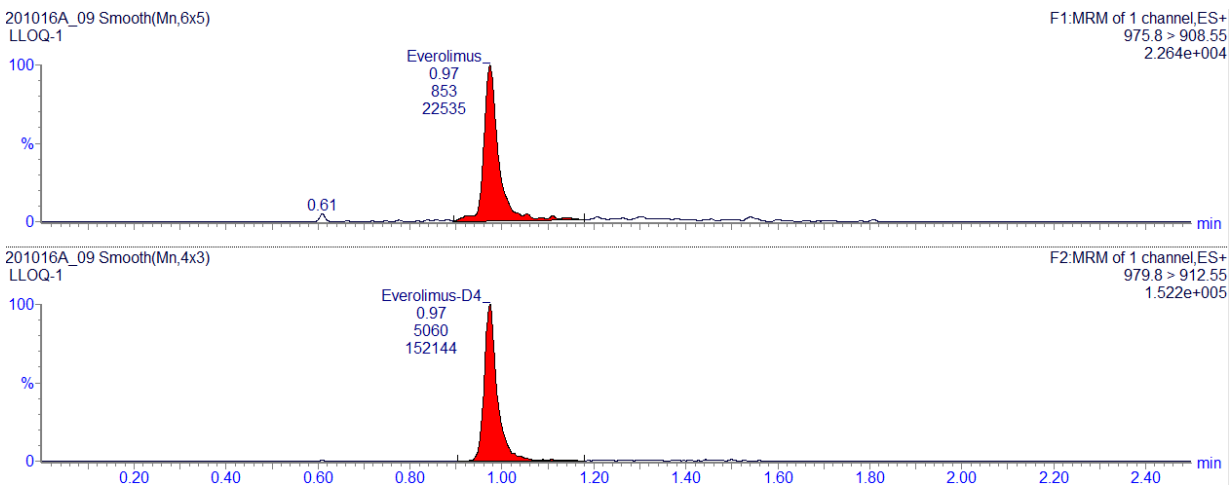

(B)

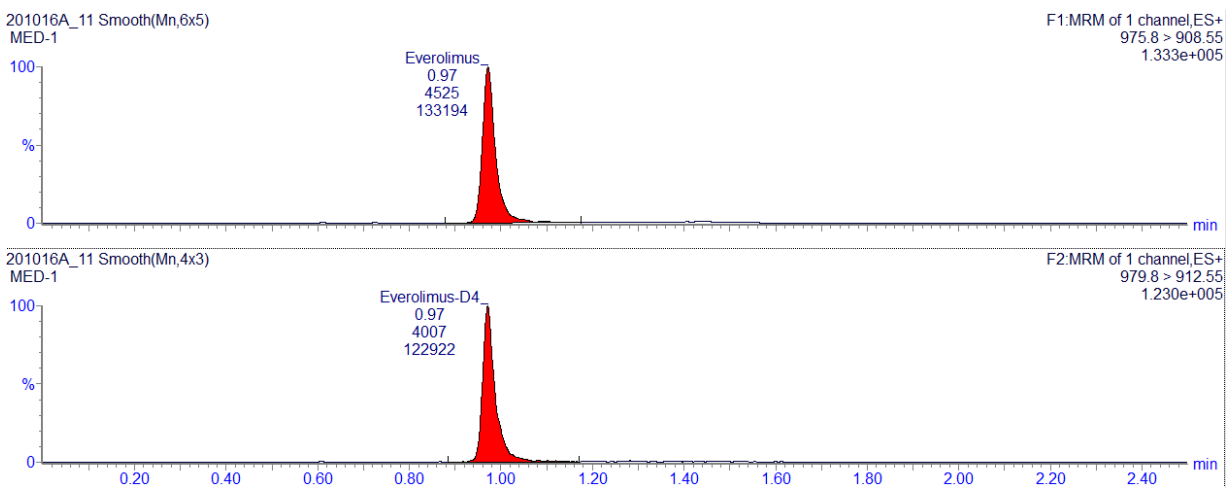

Figure 4.1.2 The typical chromatogram of respectively the LLOQ $(3 \mu \mathrm{g} / \mathrm{L})(\mathrm{A})$ and the $\mathrm{QCM}(25 \mu \mathrm{g} / \mathrm{L})(\mathrm{B})$.

Selectivity was sufficient as no interfering endogenous compounds eluting close to everolimus and IS were observed. All the responses of the six everolimus-free volunteer DBS samples were $<20 \%$ for the LLOQ and $<5 \%$ for the IS, which fell well within the acceptable limit $^{26}$.

Everolimus was validated with a linear range of 3-75 $\mu \mathrm{g} / \mathrm{L}$. The method showed a good performance in terms of linearity $\left(r^{2} \geq 0.994\right)$, as described by the following three analytical run equations: $y=0.0470 x+0.0053\left(r^{2}=0.996\right), y=0.0460 x+0.0054\left(r^{2}=0.999\right)$, and $y=0.0465 x+0.0056\left(r^{2}=0.994\right)$, where $y$ represents the peak area ratio of everolimus to that of IS and $x$ is the concentration of everolimus in DBS.

The matrix effect did not meet all acceptance criteria, since the precision at $5 \mu \mathrm{g} / \mathrm{L}$ was $15.6 \%$ (Table 4.1 .2 ). The acceptance criteria were defined as a maximum precision of the concentration and a maximum precision of the response ratio of $\leq 15 \%{ }^{26}$. The 
large precision at $5 \mu \mathrm{g} / \mathrm{L}$ was likely due to a combination of the following factors: 1) large variability inherent to a DBS method, 2) large variability inherent to the low everolimus concentration, and 3 ) the hematocrit effect. The hematocrit value of the six everolimus-free volunteer samples was verified, being $0.40,0.40,0.39,0.41,0.30$, and $0.24 \mathrm{~L} / \mathrm{L}$. After excluding the patient with a hematocrit value of $0.24 \mathrm{~L} / \mathrm{L}$, the precision at $5 \mu \mathrm{g} / \mathrm{L}$ was $14.8 \%$. Furthermore, the precision of all response ratios were $<15 \%$ (with or without excluding the patient with a hematocrit value of $0.24 \mathrm{~L} / \mathrm{L}$ ). Therefore, we expect the matrix effect to be adequate.

Table 4.1.2 Matrix effect for everolimus dried blood spot.

\begin{tabular}{|c|c|c|c|c|c|c|}
\hline $\begin{array}{l}\text { Nominal } \\
\text { concentration } \\
(\mu \mathrm{g} / \mathrm{L})\end{array}$ & $\begin{array}{c}\text { Mean } \\
\text { concentration } \\
(\mathrm{N}=6)(\mu \mathrm{g} / \mathrm{L})\end{array}$ & $\begin{array}{c}\text { Precision } \\
(\mathrm{N}=6) \\
(\mathrm{CV} \%) \\
\end{array}$ & $\begin{array}{c}\text { Precision of } \\
\text { response ratio } \\
(\mathrm{N}=6)(\mathrm{CV} \%)\end{array}$ & $\begin{array}{c}\text { Mean } \\
\text { concentration }^{\text {a }} \\
(\mathrm{N}=5)(\mu \mathrm{g} / \mathrm{L})\end{array}$ & $\begin{array}{c}\text { Precision }^{a} \\
(\mathrm{~N}=5) \\
(\mathrm{CV} \%) \\
\end{array}$ & $\begin{array}{c}\text { Precision of } \\
\text { response ratio }^{a} \\
(\mathrm{~N}=5)(\mathrm{CV} \%)\end{array}$ \\
\hline 5 & 6.0 & 15.6 & 12.4 & 6.0 & 14.8 & 11.7 \\
\hline 40 & 42.6 & 5.8 & 5.5 & 43.1 & 4.7 & 4.5 \\
\hline
\end{tabular}

$\mathrm{CV}(\%)$ : coefficient of variation; ${ }^{\text {a }}$ Patient with hematocrit value of $0.24 \mathrm{~L} / \mathrm{L}$ was excluded. The precision of the response ratio was calculated as the response of everolimus divided by the response of the internal standard. All concentrations were analysed in duplicate.

The mean recovery was $74 \%$ for the freshly prepared DBS samples and $81 \%$ for the DBS samples stored for 17 days at $2-8^{\circ} \mathrm{C}$ (Table 4.1.3). The recovery was not influenced by the hematocrit effect (i.e. $0.35 \mathrm{~L} / \mathrm{L}, 0.40 \mathrm{~L} / \mathrm{L}$ and $0.45 \mathrm{~L} / \mathrm{L}$ ). The precision at $5 \mu \mathrm{g} / \mathrm{L}$ and $0.35 \mathrm{~L} / \mathrm{L}$ for the freshly prepared DBS was $15.5 \%$, which was likely due to the large variability inherent to the low everolimus concentration in combination with the small amount of blood (i.e. $15 \mu \mathrm{L}$ ).

Carry-over was observed in 5 of the 5 blank samples of the LLOQ and the IS, however, the amount of carry-over fell well within the acceptance criteria. The acceptance criteria for the carry-over in the blank sample following the ULOQ was $\leq 20 \%$ of the LLOQ and $\leq 5 \%$ of the $\mathrm{IS}^{26}$.

Table 4.1.3 Recovery for everolimus dried blood spot depending on storage, concentration and hematocrit.

\begin{tabular}{|c|c|c|c|c|}
\hline \multirow{2}{*}{$\begin{array}{l}\text { Concentration }(\mu \mathrm{g} / \mathrm{L}) \& \\
\text { hematocrit }(\mathrm{L} / \mathrm{L})\end{array}$} & Precision (CV\%) & Recovery (\%) & Precision (CV\%) & Recovery (\%) \\
\hline & \multicolumn{2}{|c|}{ No storage } & \multicolumn{2}{|c|}{ Storage for 17 days at $2-8^{\circ} \mathrm{C}$} \\
\hline $5 \& 0.35$ & 15.5 & 76.6 & 9.2 & 92.1 \\
\hline $5 \& 0.40$ & 3.4 & 80.6 & 3.7 & 70.2 \\
\hline $5 \& 0.45$ & 9.7 & 73.6 & 13.8 & 76.9 \\
\hline $40 \& 0.35$ & 3.2 & 73.8 & 12.2 & 89.9 \\
\hline $40 \& 0.40$ & 5.9 & 69.7 & 8.3 & 80.0 \\
\hline $40 \& 0.45$ & 5.8 & 67.3 & 10.2 & 77.2 \\
\hline
\end{tabular}

$\mathrm{CV}(\%)$ : coefficient of variation. All concentrations were analysed in duplicate. 
The stability results are shown in Table 4.1.4A and B. Everolimus was proven to be stable for at least 80 days when storing at 2 to $8^{\circ} \mathrm{C}$ and for 17 days at 15 to $25^{\circ} \mathrm{C}$. Furthermore, the autosampler stability was proven sufficient for 24 hours at $10^{\circ} \mathrm{C}$, since all accuracies were within $\pm 2.7 \%$ (results not provided). The acceptance criteria for the stability were a maximum accuracy for the concentration at 5 and $50 \mu \mathrm{g} / \mathrm{L}$ of $\leq 15 \%$ as compared to the nominal concentration in addition to a maximum allowable difference of the slopes of the calibration curves of the stability samples of $\leq 15 \%$ as compared to the slope of the freshly prepared calibration curves ${ }^{26}$.

Table 4.1.4 Stability of everolimus dried blood spot samples on storage. (A) Stability assessed at the 5 and $50 \mu \mathrm{g} / \mathrm{L}$ concentration levels. (B) Influence of temperature and storage period on the slopes of the everolimus dried blood spot calibration curves.

(A)

\begin{tabular}{lccc}
\hline Temperature $\left({ }^{\circ} \mathrm{C}\right)$ & Time (days) & Accuracy $(\%)$ at $5 \mu \mathrm{g} / \mathrm{L}$ & Accuracy $(\%)$ at $50 \mu \mathrm{g} / \mathrm{L}$ \\
\hline-20 to -30 & 10 & 86.5 & 104.7 \\
-20 to -30 & 17 & 102.5 & 102.8 \\
$2-8$ & 10 & 97.6 & 97.3 \\
$2-8$ & 17 & 86.7 & 106.7 \\
$2-8$ & 40 & 89.0 & 98.2 \\
$2-8$ & 80 & 94.7 & 87.1 \\
$15-25(\mathrm{RT})$ & 10 & 89.4 & 91.3 \\
$15-25(\mathrm{RT})$ & 17 & 94.2 & 98.5 \\
$15-25(\mathrm{RT})$ & 40 & 87.3 & 82.9 \\
$15-25(\mathrm{RT})$ & 80 & 87.3 & 90.9 \\
40 & 10 & 89.3 & 79.5 \\
40 & 17 & 88.7 & 92.1 \\
\hline
\end{tabular}

RT: room temperature. Table gives mean concentration compared with nominal value. All concentrations were analysed in duplicate.

(B)

\begin{tabular}{lcccc}
\hline Temperature $\left({ }^{\circ} \mathrm{C}\right)$ & Time (days) & Intercept & Slope & Difference in slope (\%) \\
\hline-20 to -30 & 10 & -0.0190 & 0.0454 & 101.8 \\
-20 to -30 & 17 & 0.0109 & 0.0434 & 100.4 \\
$2-8$ & 10 & 0.0096 & 0.0428 & 96.0 \\
$2-8$ & 17 & 0.0365 & 0.0440 & 101.8 \\
$2-8$ & 40 & 0.0038 & 0.0410 & 91.7 \\
$2-8$ & 80 & 0.0036 & 0.0358 & 90.5 \\
$15-25(\mathrm{RT})$ & 10 & 0.0078 & 0.0399 & 89.4 \\
$15-25(\mathrm{RT})$ & 17 & 0.0086 & 0.0421 & 97.2 \\
$15-25(\mathrm{RT})$ & 40 & 0.0174 & 0.0375 & 83.8 \\
$15-25(\mathrm{RT})$ & 80 & 0.0081 & 0.0371 & 94.0 \\
40 & 10 & 0.0191 & 0.0358 & 80.3 \\
40 & 17 & 0.0023 & 0.0415 & 96.0 \\
\hline
\end{tabular}

RT: room temperature. Table gives slopes of stored calibration curves compared with the slopes of freshly prepared calibration curves. The slope of each calibration curve is based on the concentration of 6 calibrators that were analysed in duplicate. 


\section{Influence of hematocrit and concentration on accuracy}

Accuracy and precision were satisfactory at all hematocrit levels $\geq 0.25 \mathrm{~L} / \mathrm{L}$, since the precision and accuracy of the hematocrit and concentration samples should be $\leq 15 \%$ (Figure 4.1.3). At $0.20 \mathrm{~L} / \mathrm{L}$ hematocrit in combination with high everolimus concentrations of 20 and $40 \mu \mathrm{g} / \mathrm{L}$, the precision was adequate $(\leq 7.4 \%)$, however the accuracy was respectively $18.0 \%$ and $21.9 \%$ of nominal value (Figure 4.1 .3 ). The accuracies $>15 \%$ at hematocrit $0.20 \mathrm{~L} / \mathrm{L}$ in combination with the high everolimus concentrations of 20 and $40 \mu \mathrm{g} / \mathrm{L}$ may be explained by both the hematocrit effect and the relatively high number of hydrogen (H)-bond acceptors of everolimus (i.e. 14). At high everolimus concentrations, the cellulose bound fraction on the DBS paper might increase, which might result in lower recoveries ${ }^{24}$.

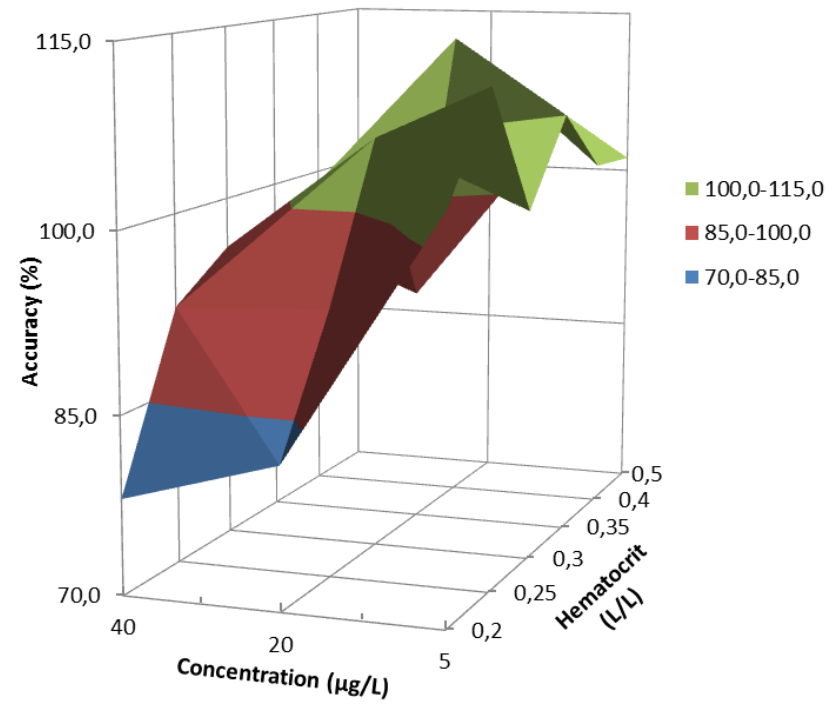

Figure 4.1.3 The accuracy for everolimus dried blood spot influenced by hematocrit and concentration.

\section{Influence of hematocrit and spot volume on difference in slope}

At $0.40 \mathrm{~L} / \mathrm{L}$ hematocrit, the blood spot volume showed to be of minor impact on the measured concentrations, illustrated by differences of $\leq 3.5 \%$ for all slopes (Table 4.1.5). At $0.20 \mathrm{~L} / \mathrm{L}$ hematocrit, the differences of the slopes of the $20 \mu \mathrm{L}, 30 \mu \mathrm{L}, 40 \mu \mathrm{L}$ and $50 \mu \mathrm{L}$ calibration curves were $14.6 \%, 21.7 \%, 14.3 \%$ and $16.4 \%$, respectively. The large differences of the slopes at $0.20 \mathrm{~L} / \mathrm{L}$ hematocrit can likely be explained by the 
hematocrit effect. Blood with a lower viscosity spreads further on the DBS sampling paper, causing large differences of the slopes since fixed DBS punching areas were measured $^{19,23}$. The maximum allowable difference of the slopes of the calibration curves at $0.20 \mathrm{~L} / \mathrm{L}$ and $0.40 \mathrm{~L} / \mathrm{L}$ hematocrit sampled with volumes of $20-50 \mu \mathrm{L}$ should be $\leq 15 \%$ as compared to the slope of the calibration curve at $0.40 \mathrm{~L} / \mathrm{L}$ hematocrit sampled with a volume of $30 \mu \mathrm{L}$.

Table 4.1.5 Influence of hematocrit and blood spotting volume on the slopes of the everolimus dried blood spot calibration curves.

\begin{tabular}{lcccc}
\hline Hematocrit $(\mathrm{L} / \mathrm{L})$ & Blood spot volume $(\mu \mathrm{L})$ & Intercept & Slope & Difference in slope $(\%)$ \\
\hline 0.20 & 20 & 0.0371 & 0.0287 & 85.4 \\
0.20 & 30 & 0.0439 & 0.0263 & 78.3 \\
0.20 & 40 & 0.0293 & 0.0288 & 85.7 \\
0.20 & 50 & 0.0342 & 0.0281 & 83.6 \\
0.40 & 20 & 0.0369 & 0.0341 & 101.4 \\
0.40 & 30 & 0.0535 & 0.0336 & Reference \\
0.40 & 40 & 0.0670 & 0.0324 & 96.5 \\
0.40 & 50 & 0.0686 & 0.0337 & 100.2 \\
\hline
\end{tabular}

Table gives slopes of calibration curves assessed with spot volumes of $20,30,40,50 \mu \mathrm{L}$ at the hematocrit levels of 0.20 and $0.40 \mathrm{~L} / \mathrm{L}$ compared with the slope of a calibration curve assessed with $30 \mu \mathrm{L}$ spot volume at hematocrit $0.4 \mathrm{~L} / \mathrm{L}$ (=reference). The slope of each calibration curve is based on the concentration of 6 calibrators that were analysed in duplicate.

In conclusion, the everolimus DBS sampling method has been successfully analytically validated for whole-blood over a range of 3-75 $\mu \mathrm{g} / \mathrm{L}$. As compared to previous published everolimus DBS methods, the total analysis time of this method is short (3.5 min versus 6.5 and $4.2 \mathrm{~min}$, respectively) ${ }^{12,19}$, and the method does not require an impregnation $\operatorname{step}^{12}$. The hematocrit accuracy and precision were satisfactory at hematocrit values $\geq 0.25 \mathrm{~L} / \mathrm{L}$, however, not satisfactory at $0.20 \mathrm{~L} / \mathrm{L}$ hematocrit at the high everolimus concentrations of 20 and $40 \mu \mathrm{g} / \mathrm{L}$. Correction for the hematocrit effect at $0.20 \mathrm{~L} / \mathrm{L}$ can be achieved by preparing a full set of calibration standards at $0.20 \mathrm{~L} / \mathrm{L}$ when the hematocrit value of the samples is expected or measured to be close to $0.20 \mathrm{~L} / \mathrm{L}^{28}$. An alternative method could be analysis of the whole blood spot (instead of centre punch), or by using volumetric absorptive microsampling, since these methods analyse a fixed volume of blood ${ }^{29,30}$. The validated everolimus assay is currently used in a clinical pharmacokinetic study for the use of everolimus DBS in cancer patients. 


\section{References}

1. European Medicinces Agency. Afinitor, Summary of Product Characteristics. 2009. Retrieved from https://www.ema.europa.eu/documents/pro duct-information/afinitor-epar-productinformation_en.pdf. Accessed February 2017.

2. Yao JC, Fazio N, Singh S, et al. Everolimus for the treatment of advanced, non-functional neuroendocrine tumours of the lung or gastrointestinal tract (RADIANT-4): a randomised, placebo-controlled, phase 3 study. Lancet. 2016;387(10022):968-77.

3. Yao JC, Shah MH, Ito T, et al. Everolimus for advanced pancreatic neuroendocrine tumors. N Engl J Med. 2011;364(6):514-23.

4. Motzer RJ, Escudier B, Oudard S, et al. Efficacy of everolimus in advanced renal cell carcinoma: a double-blind, randomized, placebo-controlled phase III trial. Lancet. 2008;372(9637):449-56.

5. Baselga J, Campone $M$, Piccart $M$, et al. Everolimus in postmenopausal hormonereceptor-positive advanced breast cancer. N Engl J Med. 2012;366(6):520-9.

6. US Food \& Drug Administration. Center for Drug Evaluation and Research. Application Number: 22-334. Clinical Pharmacology and Biopharmaceutics Review(s). Afinitor. 2008. Retrieved from https://www.accessdata.fda.gov/drugsatfda_ docs/nda/2009/022334s000_ClinPharmR.pdf. Accessed March 2017.

7. Shipkova M, Hesslink DA, Holt DW, et al. Therapeutic Drug Monitoring of Everolimus: A Consensus Report. Ther Drug Monit. 2016;38(2):143-69.

8. de Wit D, Schneider TC, Moes DJ, et al. Everolimus pharmacokinetics and its exposure-toxicity relationship in patients with thyroid cancer. Cancer Chemother Pharmacol. 2016;78(1):63-71.

9. Ravaud A, Urva SR, Grosch K, et al. Relationship between everolimus exposure and safety and efficacy: meta-analysis of clinical trials in oncology. Eur J Cancer. 2014;50(3):486-95.

10. Thiery-Vuillemin A, Mouillet G, Nguyen Tan Hon $\mathrm{T}$, et al. Impact of everolimus blood concentration on its anti-cancer activity in patients with metastatic renal cell carcinoma.
Cancer Chemother Pharmacol. 2014;73(5): 999-1007.

11. Deppenweiler M, Falkowski S, Saint-Marcoux $F$, et al. Towards therapeutic drug monitoring of everolimus in cancer? Results of an exploratory study of exposure-effect relationship. Pharmacol Res. 2017;121:138144.

12. van der Heijden J, de Beer $Y$, Hoogtanders $K$, et al. Therapeutic drug monitoring of everolimus using the dried blood spot method in combination with liquid chromatographymass spectrometry. J Pharm Biomed Anal. 2009;50(4):664-70.

13. Koster RA, Alffenaar JW, Greijdanus B, et al. Fast LC-MS/MS analysis of tacrolimus, sirolimus, everolimus and cyclosporin $A$ in dried blood spots and the influence of the hematocrit and immunosuppressant concentration on recovery. Talanta. 2013;115:47-54.

14. Hoogtanders K, van der Heijden J, Christiaans $M$, et al. Dried blood spot measurement of tacrolimus is promising for patient monitoring. Transplantation. 2007;83(2):2378.

15. Nijenhuis CM, Huitema AD, Marchetti S, et al. The Use of Dried Blood Spots for Pharmacokinetic Monitoring of Vemurafenib Treatment in Melanoma Patients. J Clin Pharmacol. 2016;56(10):1307-12.

16. de Wit D, den Hartigh J, Gelderblom $\mathrm{H}$, et al. Dried blood spot analysis for therapeutic drug monitoring of pazopanib. J Clin Pharmacol. 2015;55(12):1344-50.

17. Kralj E, Trontelj J, Pajič T, et al. Simultaneous measurement of imatinib, nilotinib and dasatinib in dried blood spot by ultra high performance liquid chromatography tandem mass spectrometry. J Chromatogr B Analyt Technol Biomed Life Sci. 2012;903:150-6.

18. Jager NG, Rosing $\mathrm{H}$, Schellens $\mathrm{JH}$, et al. Use of dried blood spots for the determination of serum concentrations of tamoxifen and endoxifen. Breast Cancer Res Treat. 2014; 146(1):137-44.

19. den Burger JC, Wilhelm AJ, Chahbouni A, et al. Analysis of cyclosporin A, tacrolimus, sirolimus, and everolimus in dried blood spot samples using liquid chromatography tandem 
mass spectrometry. Anal Bioanal Chem 2012; 404(6-7):1803-11.

20. Koster RA, Veenhof $H$, Botma R, et al. Dried blood spot validation of five immunosuppressants, without hematocrit correction, on two LC-MS/MS systems. Bioanalysis. 2017;9(7):553-63.

21. van Erp NP, van Herpen $C M$, de Wit $D$, et al. $A$ Semi-Physiological Population Model to Quantify the Effect of Hematocrit on Everolimus Pharmacokinetics and Pharmacodynamics in Cancer Patients. Clin Pharmacokinet. 2016;55(11):1447-56.

22. Knight K, Wade S, Balducci L. Prevalence and outcomes of anemia in cancer: a systematic review of the literature. Am J Med. 2004;116Suppl7A:11S-26S.

23. Wagner M, Tonoli D, Varesio E, et al. The use of mass spectrometry to analyze dried blood spots. Mass Spectrom Rev. 2016;35(3):361438.

24. Koster RA, Alffenaar JW, Botma R, et al. The relation of the number of hydrogen-bond acceptors with recoveries of immunosuppressants in DBS analysis. Bioanalysis. 2015;7(14):1717-22.

25. Capone D, Gentile A, Polichetti G et al. Stability of sirolimus and everolimus measured by immunoassay techniques in whole blood samples from kidney transplant patients. Int J Immunopathol Pharmacol. 2008;21(2):297-307.
26. European Medicines Agency. Guideline on bioanalytical method validation. 21 July 2011. Retrieved from https://www.ema.europa.eu/documents/scie ntific-guideline/guideline-bioanalyticalmethod-validation_en.pdf. Accessed March 2017.

27. Jager NG, Rosing $H$, Schellens $J H$, et al. Procedures and practices for the validation of bioanalytical methods using dried blood spots: a review. Bioanalysis. 2014;6(18):2481-514.

28. Wilhelm AJ, den Burger JC, Swart EL. Therapeutic drug monitoring by dried blood spot: progress to date and future directions. Clin Pharmacokinet. 2014;53(11):961-73.

29. Kip $A E$, Kiers $K C$, Rosing $H$, et al. Volumetric absorptive microsampling (VAMS) as an alternative to conventional dried blood spots in the quantification of miltefosine in dried blood samples. J Pharm Biomed Anal. 2017; 135:160-66.

30. Zheng N, Yuan L, Ji QC, et al. "Center punch" and "whole spot" bioanalysis of apixaban in human dried blood spot samples by UHPLCMS/MS. J Chromatogr B Analyt Technol. Biomed Life Sci. 2015;988:66-74. 


\section{Chapter}

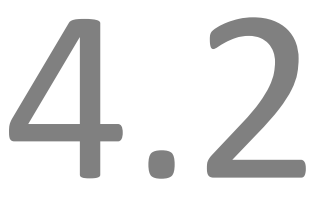

\section{Clinical validation study of dried blood spot for determining everolimus concentration in patients with cancer}

A.E.C.A.B. Willemsen, L.M. Knapen, Y.M. de Beer, R.J.M. Brüggemann, S. Croes,

C.M.L. van Herpen, N.P. van Erp

Eur J Clin Pharmacol. 2018;74(4):465-471 


\section{Abstract}

\section{Background}

Everolimus treatment is seriously hampered by its toxicity profile. As a relationship between everolimus exposure and effectiveness and toxicity has been established, early and ongoing concentration measurement can be key to individualise the dose and optimise treatment outcomes. Dried blood spot (DBS) facilitates sampling at a patients' home and thereby eases dose individualisation.

\section{Objectives}

The aim of this study is to determine the agreement and predictive performance of DBS compared to whole-blood (WB) to measure everolimus concentrations in cancer patients.

\section{Patients and methods}

Paired DBS and WB samples were collected in 22 cancer patients treated with everolimus and analysed using UPLC-MS/MS. Bland-Altman and Passing-Bablok analysis were used to determine method agreement. Limits of clinical relevance were set at a difference of $\pm 25 \%$, as this would lead to a different dosing advice. Using DBS concentration and Passing-Bablok regression analysis, WB concentrations were predicted.

\section{Results}

Samples of 20 patients were suitable for analysis. Bland-Altman analysis showed a mean ratio of everolimus WB to DBS concentrations of 0.90 , with $95 \%$ of data points within limits of clinical relevance. Passing-Bablok regression of DBS compared to WB revealed no constant bias (intercept 0.02; 95\% confidence interval (Cl) 0.93-1.35) and a small proportional bias (slope 0.89; $95 \% \mathrm{Cl}$ 0.76-0.99). Predicted concentrations showed low bias and imprecision, and $90 \%$ of samples had an absolute percentage prediction error of $<20 \%$.

\section{Conclusions}

DBS is a valid method to determine everolimus concentrations in cancer patients. This can especially be of value for early recognition of over- or underexposure to enable dose adaptations. 


\section{Introduction}

The introduction of everolimus has brought significant benefit for patients with metastatic renal cell carcinoma ( $\mathrm{mRCC}$ ), advanced hormone receptor-positive/human epidermal growth factor receptor 2-negative breast cancer, and advanced or unresectable neuroendocrine tumours of pancreatic, gastrointestinal, or lung origin ${ }^{1-4}$. Currently, the treatment with everolimus in patients with cancer is not individualised and no therapeutic drug monitoring (TDM) is routinely being performed. The standard initial dose is $10 \mathrm{mg}$ orally once daily, which may be reduced in case of toxicity or fragility ${ }^{5}$. This practice is in contrast to solid organ transplantation medicine, where doses of 0.75 to $1.0 \mathrm{mg}$ twice daily are used and where TDM of everolimus, including dried blood spot (DBS) monitoring, to guide dosing has been incorporated in the standard care for over 10 years ${ }^{6,7}$. Also, everolimus treatment of subependymal giant cell astrocytoma with tuberous sclerosis complex is individualised based on TDM ${ }^{8}$.

Several arguments point to the use of TDM for everolimus in patients with cancer as well. A relationship between everolimus drug exposure and effectiveness and safety has been established in several studies ${ }^{9-12}$. Everolimus trough concentrations above $11.9 \mu \mathrm{g} / \mathrm{L}$ and below $26.3 \mu \mathrm{g} / \mathrm{L}$, respectively, were associated with a threefold increase in progression-free survival and a fourfold decreased risk of toxicity in patients with breast cancer, kidney cancer, and neuroendocrine cancer ${ }^{10}$. Larger studies are required to further define the optimal therapeutic window. Everolimus has an interpatient pharmacokinetic variability up to $36-45 \%$ and shows dose proportional pharmacokinetics over the range of 5 to $10 \mathrm{mg}$ once daily ${ }^{5,9,13-15}$. Given the exposureeffectiveness relationship, the narrow therapeutic index, the large interpatient variability, and linear pharmacokinetics, it seems important to guide everolimus dosing pharmacokinetically.

If TDM of everolimus is performed in patients with cancer, sampling with DBS can bring many advantages over venous sampling, as it is minimally invasive, simple, and flexible. After adequate training and with clear instructions, patients can perform DBS at home and sent their sample by regular mail to the laboratory for analysis. Also, physicians may benefit from the ease of the DBS sampling method, as it can provide them with analysis results before patients visit the outpatient clinic for their (routine) check-up ${ }^{7}$. As such, DBS is a promising alternative to venous sampling and it already has become increasingly common in other anticancer drugs ${ }^{16-19}$. The use of DBS to predict venous whole-blood (WB) everolimus concentration has been established for organ transplantation patients, in which the administered dosage of everolimus is much lower than in cancer patients ${ }^{6,7,20,21}$. In patients with cancer, the agreement between everolimus DBS concentrations and WB concentrations is yet unknown. In order to enhance the implementation of DBS in clinical practice, this is the first clinical validation study, in which we aim to determine the agreement and the predictive performance of DBS compared to WB to measure everolimus concentrations in patients with cancer. 


\section{Methods}

\section{Study population}

The current study is an observational pharmacokinetic study in patients aged $>18$ years, treated with everolimus for any type of solid tumour at the Radboud university medical centre (Radboudumc), an academic hospital in the Netherlands. No exclusion criteria were set since the study population intends to reflect a 'real-life' group of patients with cancer treated with everolimus. As such, the dose and duration of everolimus treatment was not restricted for inclusion.

\section{Sampling and everolimus concentration}

Each patient was sampled in the outpatient clinic during their routine follow-up, at one moment, while being on steady state (i.e. treated for at least 7 days). Patients were asked not to take everolimus at the day of the visit at home, but only directly after obtaining the WB and DBS samples. Two drops of capillary blood were sampled on the sampling paper in order to create the DBS samples in duplicate. To establish whether the difference in blood source (capillary versus whole-blood) is a cause of variation, an additional DBS was made from a drop of whole-blood (DBS $\mathrm{wb}_{\mathrm{w}}$ ).

Since hematocrit might affect the quantification of everolimus in DBS due to inhomogeneity of the droplet on the paper, the hematocrit value of the WB samples was determined at the day the venipuncture and DBS took place. All samples per patient were collected within 10 minutes of each other by a dedicated physician.

Time after drug administration (interval between last dose intake and sampling) and dosing scheme were documented to estimate everolimus trough concentration $\left(C_{\text {trough }}\right)$, as described by Wang et al. ${ }^{22}$.

\section{Bioanalysis}

The DBS and DBS ${ }_{\mathrm{wb}}$ samples were visually inspected and scored whether the spot size was adequately shaped and sized for analysis. If both spots were of correct size, the average concentration of the two samples was used. If both spots were of incorrect size, these samples were not used for further analysis. After scoring of spot size, a 7.5-mm disk from the central part of the blood spot was punched out from the sample paper. Bioanalysis of the $W B, D B S$ and $D_{B S}$ wb samples was performed using two validated ultra performance liquid chromatography-tandem mass spectrometry (UPLC-MS/MS) methods. One method was used for the analysis of WB samples and another method was used to analyse the DBS and DBS ${ }_{\text {wb }}$ samples ${ }^{23}$. 


\section{Statistical analysis}

The clinical and laboratory standards institute advises to study 40 samples for agreement analysis ${ }^{24}$. However, as everolimus is measured in WB and therefore in the same matrix as DBS and as no effect of hematocrit is expected, the expected variation in DBS measurements is smaller than in other DBS studies. Moreover, in transplantation patients, good agreement of everolimus measurement between DBS and WB has been shown previously ${ }^{21}$. Therefore, we performed a power analysis for Bland-Altman analysis to determine the sample size $\mathrm{e}^{25}$. Assuming an expected mean of difference of $9 \%$, with a standard deviation of $5 \%$ and a maximum allowed difference of $25 \%$, and $\alpha$ of 0.05 and power of 0.80 , we required samples of 20 patients. Two extra patients were recruited for the risk of invalid samples.

To study the level of agreement between everolimus concentrations in DBS, DBS and WB, Bland-Altman analysis was performed ${ }^{26}$. In this analysis, we set limits of clinical relevance on a $25 \%$ range around the ratio of the two measurements. This range was chosen, as everolimus can be dose-adjusted in steps of $25 \%$ of the total dose. Passing-Bablok regression analysis was performed to detect constant and proportional bias, by analysing the intercept and the slope of the regression line, respectively ${ }^{27}$.

Furthermore, the DBS everolimus concentration was used to predict the measured WB concentration. With Passing-Bablok regression, the intercept and slope were determined using the whole population while excluding the data of the individual patient from whom the WB everolimus concentration is to be predicted. Subsequently, the intercept and slope were used to predict the WB everolimus concentration, based on the DBS concentration. This process was repeated for each individual patient. For analysing the predictive performance, the following equations from the guideline of Sheiner and $\mathrm{Beal}^{28}$ were used.

For bias:

median prediction error $(M P E)=$ median $($ WBpred $-W B)$

median percentage prediction error (MPPE) - median [100\% $\times($ WBpred $-W B) \div W B]$ (2)

For imprecision:

root median squared prediction error $(R M S E)=V$ median $(W B p r e d-W B)^{2}$

median absolute percentage prediction error $(M A P E)=$ median $[100 \% \times /($ WBpred $-(4)$ $W B) \mid \div W B]$

Values of median percentage prediction error (MPPE) and median absolute percentage prediction error (MAPE) of $<15 \%$ were considered acceptable. Overall predictive performance was measured by the percentage of samples with an absolute percentage prediction error of $<20 \%$. We set the criterion that at least $67 \%$ of samples 
should have a prediction error of $<20 \%$, analogous to the criteria for cross validation of the European Medicines Agency (EMA) guideline on bioanalytical method validation ${ }^{29}$.

All calculations were performed using Microsoft Office Excel (Microsoft Inc., Redmond, WA) and add-in Analyse-it statistics software, version 4.10.2 (Analyse-it Software, Ltd., Leeds, UK).

\section{Results}

\section{Patients and everolimus concentrations}

As planned, 22 patients were included, from June to December 2015. WB, DBS, and DBS $_{w b}$ samples of 20 patients were included in the final analysis, since one set of samples were lost and the duplicate DBS samples of one patient was insufficient for analysis. The population consisted of 11 patients with advanced breast cancer and 9 patients with mRCC. The median age at index date was 62.0 years (range 38- 73) and the median everolimus dose was $10 \mathrm{mg}$ (range 5-17.5 mg). The median hematocrit value of the WB samples was $0.35 \mathrm{~L} / \mathrm{L}$ (range 0.25-0.45) (Table 4.2.1). The everolimus concentrations of the 20 analysed samples ranged from 3.7 to $33.3 \mu \mathrm{g} / \mathrm{L}$ in DBS, from 3.3 to $31.2 \mu \mathrm{g} / \mathrm{L}$ in $\mathrm{DBS}_{\mathrm{wb}}$ and from 3.6 to $28.5 \mu \mathrm{g} / \mathrm{L}$ in WB. When the $\mathrm{C}_{\text {trough }}$ concentrations were calculated for the individual patients using the equation of Wang et al., $55 \%$ of patients had a $\mathrm{C}_{\text {trough }}$ concentration below $11.9 \mu \mathrm{g} / \mathrm{L}$ and $15 \%$ a $\mathrm{C}_{\text {trough }}$ concentration above $26.3 \mu \mathrm{g} / \mathrm{L}^{22}$.

Table 4.2.1 Patient baseline characteristics ${ }^{a}$.

\begin{tabular}{lc}
\hline Baseline characteristics & $\mathrm{N}=20$ \\
\hline Age (years) & $62(38-73)$ \\
Sex (number, \%) & $8(40)$ \\
$\quad$ Male & $12(60)$ \\
Female & $72.4(48.5-90.2)$ \\
Weight (kg) & $0.35(0.25-0.45)$ \\
Hematocrit (L/L) & $10.0(5.0-17.5)$ \\
Everolimus daily dose (mg) & \\
Tumour type (number, \%) & $11(55)$ \\
Breast cancer & $9(45)$ \\
Renal cell carcinoma & \\
\hline
\end{tabular}

${ }^{\text {a }}$ Data are median (range), unless stated otherwise.

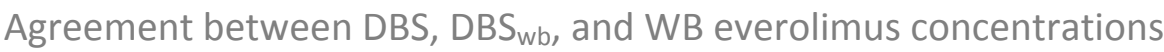

Bland-Altman analysis was used to determine the level of agreement between DBS, $D_{B b}$, and WB everolimus concentrations. The Bland-Altman plot with $95 \%$ limits of agreement (LOA) showed a small and balanced spread of relative differences between DBS and WB everolimus concentrations, with a mean ratio of everolimus WB to DBS 
concentrations of 0.90 (95\% LoA $0.71-1.08)$. Only $1 / 20$ (5\%) of values fell outside the limits of agreement and outside the limits of clinical relevance (Figure 4.2.1). Using Passing-Bablok regression analysis, a strong linear relationship was found between both methods, with a correlation coefficient of $r=0.97$, thus explaining $95 \%$ of the variance $\left(r^{2}=0.95\right)$. No significant constant bias was found, with an intercept close to zero (intercept estimate $0.02 \mu \mathrm{g} / \mathrm{L} ; 95 \% \mathrm{Cl}-0.93-1.35$ ), and only a small proportional bias (slope estimate 0.89; 95\% Cl 0.76-0.99) (Figure 4.2.2).

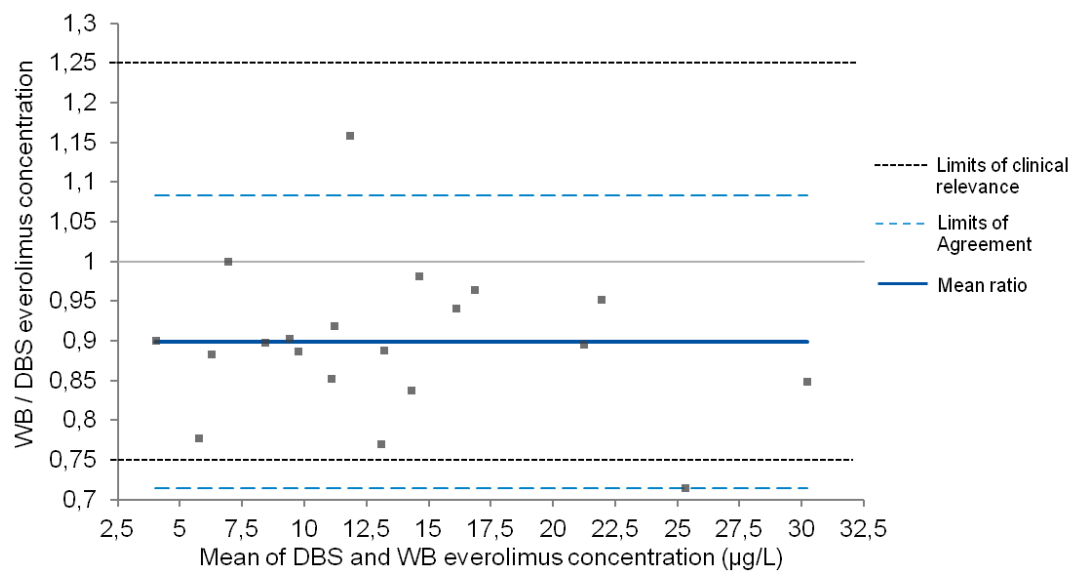

Figure 4.2.1 Bland-Altman plot of ratio between whole-blood and dried blood spot everolimus concentrations versus mean everolimus concentration.

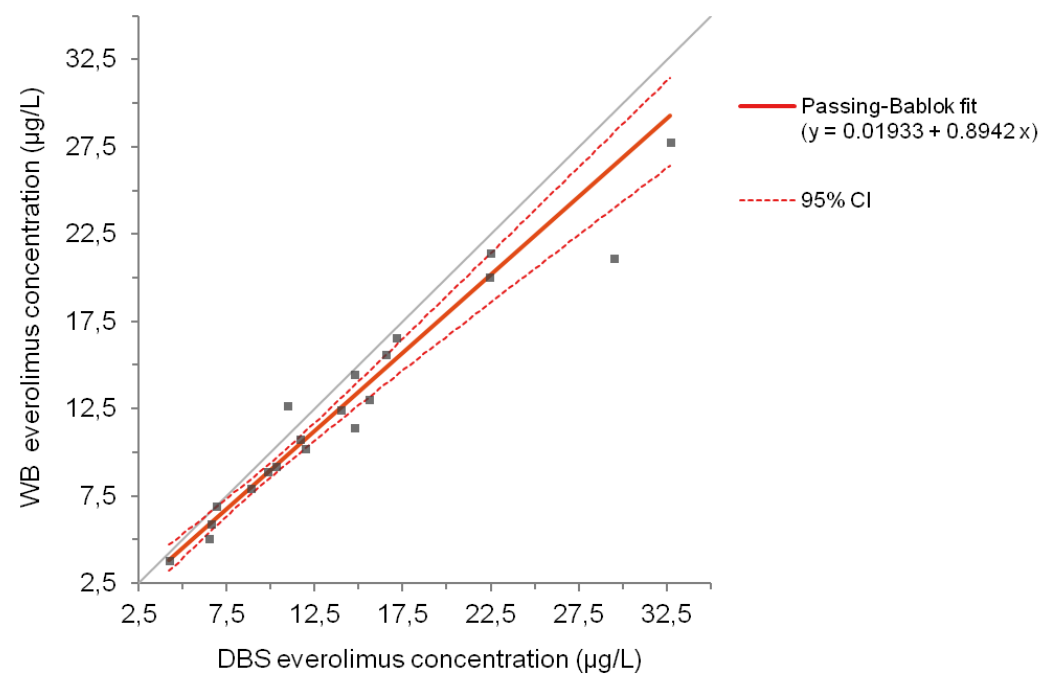

Figure 4.2.2 Passing-Bablok plot of everolimus concentrations from dried blood spot and whole-blood. 
Agreement between $\mathrm{DBS}_{\mathrm{wb}}$ and WB everolimus concentrations was comparable to the results of the DBS and WB analysis. Bland-Altman analysis showed a mean ratio of everolimus $\mathrm{DBS}_{\mathrm{wb}}$ concentrations to WB concentrations of 0.92 (95\% LoA 0.79-1.05). Passing-Bablok regression showed a coefficient of determination $r^{2}=0.98$, no constant bias (intercept $-0.17 \mu \mathrm{g} / \mathrm{L} ; 95 \% \mathrm{Cl}-1.37-0.51$ ), and no proportional bias (slope estimate $0.93 ; 95 \% \mathrm{Cl} 0.87-1.04$ ).

Everolimus concentrations of DBS and $\mathrm{DBS}_{\mathrm{wb}}$ were similar, as the Bland-Altman plot showed a mean ratio of nearly 1 (mean ratio $0.98 ; 95 \%$ LoA $0.82-1.13$ ). The Passing-Bablok regression of the two DBS sampling methods showed a coefficient of determination $r^{2}=0.97$, no constant bias (intercept $0.46 \mu \mathrm{g} / \mathrm{L} ; 95 \% \mathrm{Cl}-0.71-2.40$ ) and no proportional bias (slope estimate, $0.93 ; 95 \% \mathrm{Cl} 0.80-1.03$ ).

\section{Predictive performance of predicted everolimus WB concentrations compared with measured WB}

Based on the DBS concentrations and using the intercept and slope, everolimus WB concentrations were predicted. The total error of this prediction is determined by bias (average difference between estimator and true value) and imprecision (variance of the estimator). Bias between the predicted and the measured everolimus concentration was negligible, with median absolute difference as measured by median prediction error (MPE) of only $0.015 \mu \mathrm{g} / \mathrm{L}$, and median relative difference as measured by MPPE of $0.035 \%$. The imprecision of the predicted concentration was small, with root median squared prediction error (RMSE) of $0.76 \mu \mathrm{g} / \mathrm{L}$ and MAPE of $6.1 \%$. These values are well within the acceptable limits of $15 \%$. Overall predictive performance was good, as $90 \%$ of samples had an absolute percentage prediction error of $<20 \%$ (Figure 4.2 .3 ), which fell within the criterion of at least $67 \%$ of samples ${ }^{29}$.

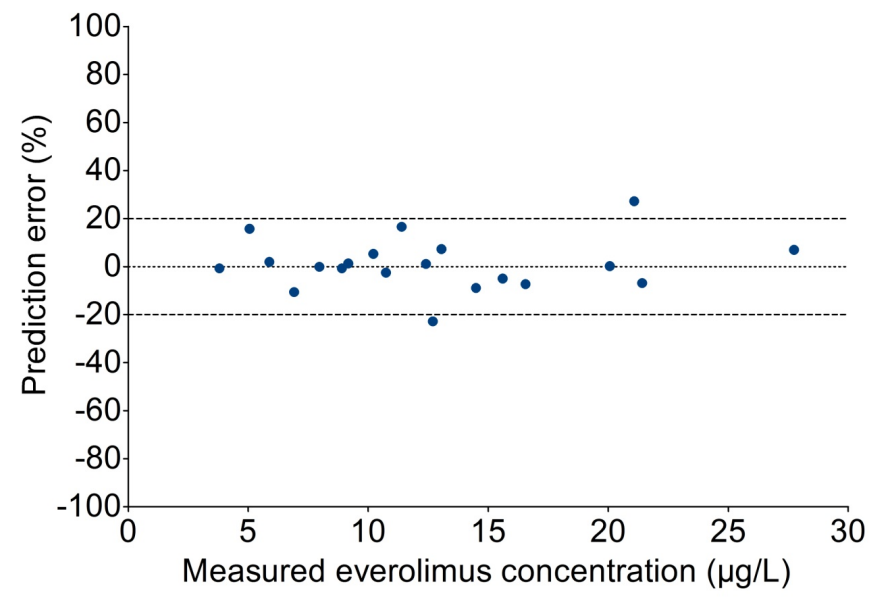

Figure 4.2.3 Percentage prediction error of predicted to measured everolimus concentration. 


\section{Discussion}

In this study, we have shown that the agreement of DBS with WB concentration measurements of everolimus in patients with cancer is very high over the entire concentration range. With DBS, results of $95 \%$ of samples fell within the limits of clinical significance, which would thereby lead to the same dosing advice as when WB measurement was used. The predictive performance of DBS was excellent, with negligible bias and small imprecision and good overall predictive performance, satisfying the EMA criteria for cross validation ${ }^{29,30}$. Consequently, in view of the high agreement and excellent predictive performance, DBS is a valid method as a practical alternative for venous sampling to measure everolimus concentrations in patients with cancer. As such, these results confirm the results of a DBS validation study of everolimus in 55 transplant patients, where a similar slope and a somewhat larger intercept was found ${ }^{21}$.

DBS $_{w b}$ was determined in order to discriminate influences of the blood drop collection methods (capillary versus whole-blood) and material (filter paper versus EDTA tube). As DBS and DBS ${ }_{w b}$ everolimus concentrations were nearly similar, it can be concluded that collection of blood by finger prick does not affect concentration measurements of everolimus.

Albeit not significantly, DBS and $\mathrm{DBS}_{\mathrm{wb}}$ did show consistently higher everolimus concentrations compared to measuring everolimus in WB. Everolimus is strongly bound to erythrocytes (approximately $85 \%$ at the blood concentration range of $5-100 \mu \mathrm{g} / \mathrm{L})^{31,32}$, and we speculate that this fraction can have a higher concentration at the centre of the punch of the sampling paper. When hematocrit levels are low $(\leq 0.20 \mathrm{~L} / \mathrm{L})$, chromatographic effects can play a role, resulting in inaccurately lower everolimus concentration measurements ${ }^{33}$. Especially DBS samples with high everolimus concentrations ( $>20 \mu \mathrm{g} / \mathrm{L}$ ) in combination with low hematocrit can provide inaccurate lower results ${ }^{21,34}$. As hematocrit levels were $\geq 0.25 \mathrm{~L} / \mathrm{L}$ for all patients, the impact of hematocrit on everolimus concentration measurement was not an issue in the population we investigated. However, awareness should be in place for everolimus measurements in patients with a hematocrit below $0.20 \mathrm{~L} / \mathrm{L}$. The collection of blood spots by volumetric absorptive microsampling is an alternative for DBS sampling that might overcome this issue, as it enables the collection of an accurate blood volume, independently of hematocrit levels ${ }^{35}$.

It is important to note certain limitations of this study. First, the finger prick sampling was performed by a dedicated physician at the hospital, and since this situation does not reflect the at-home oncology sampling setting, the results cannot be extrapolated without further validation. Therefore, we recommend future studies to perform a validation of clinical utility with DBS cards sampled by patients themselves. However, we expect at-home sampling to be feasible when clear instructions and adequate training are provided, since previous literature has shown that 86 to $98 \%$ of 
the DBS samples obtained from patients were suitable for analysis ${ }^{36-38}$. Second, the number of analysed DBS was relatively small. A smaller sample size leads to a wider confidence interval, resulting in a decreased power to detect a difference between two methods. However, this sample size is comparable to the sample size in other studies with DBS and oral anticancer agents ${ }^{16,39}$, while the agreement between DBS with WB for everolimus is markedly better than for other anticancer drugs, such as pazopanib and nilotinib ${ }^{16,39}$. DBS shows a relatively high performance to measure everolimus concentrations, when compared to the performance of DBS for measuring other drugs. This can partially be explained as everolimus is measured in WB instead of plasma, and therefore has the same matrix as DBS. Moreover, good agreement of DBS and WB has been shown for everolimus in transplantation patients ${ }^{21}$. Therefore, we considered it appropriate to base our sample size on a power calculation using previously obtained data in transplantation patients treated with everolimus for our assumptions. This deems to be a legitimate approach, since our data indeed show highly similar results to the previous independently performed study. Third, only trough levels were analysed. This did not lead to restrictions, however, as a wide range of everolimus concentrations were obtained.

In summary, it can be concluded that this is the first study that demonstrates the clinical validity of DBS sampling for analysis of everolimus concentration in patients with cancer. Measuring everolimus concentrations in patients with cancer is particularly important to identify patients with very low or high everolimus concentrations. In our study $70 \%$ of our patients had extrapolated everolimus trough concentrations that fell outside the proposed therapeutic window. In these patients, it is likely that dose adjustments can improve effectiveness or prevent toxicity. DBS is a patient-friendly and practical alternative for WB concentration measurements and in this study has shown to be accurate for the goal to individualise everolimus therapy in cancer patients. Implementation of standard everolimus DBS measurement early after start of treatment has the potential to improve clinical outcomes for patients with cancer treated with everolimus. 


\section{References}

1 Motzer RJ, Escudier B, Oudard S, et al. Phase 3 trial of everolimus for metastatic renal cell carcinoma: final results and analysis of prognostic factors. Cancer. 2010;116(18):4256-65.

2 Baselga J, Campone $M$, Piccart $M$, et al. Everolimus in postmenopausal hormonereceptor-positive advanced breast cancer. $\mathrm{N}$ Engl J Med. 2012;366(6):520-9.

3 Yao JC, Fazio N, Singh S, et al, Rad001 in Advanced Neuroendocrine Tumours FTSG. Everolimus for the treatment of advanced, non-functional neuroendocrine tumours of the lung or gastrointestinal tract (RADIANT-4): a randomised, placebo-controlled, phase 3 study. Lancet. 2016;387(10022):968-77.

4 Yao JC, Shah MH, Ito T, et al, Rad001 in Advanced Neuroendocrine Tumors TTSG. Everolimus for advanced pancreatic neuroendocrine tumors. N Engl J Med. 2011;364(6):514-23.

5 European Medicinces Agency. Afinitor, Summary of Product Characteristics. 2009. Retrieved from https://www.ema.europa.eu/ documents/product-information/afinitorepar-product-information_en.pdf. Accessed February 2017.

6 den Burger JC, Wilhelm AJ, Chahbouni A, et al. Analysis of cyclosporin A, tacrolimus, sirolimus, and everolimus in dried blood spot samples using liquid chromatography tandem mass spectrometry. Anal Bioanal Chem. 2012;404(6-7):1803-11.

7 van der Heijden J, de Beer Y, Hoogtanders K, et al. Therapeutic drug monitoring of everolimus using the dried blood spot method in combination with liquid chromatographymass spectrometry. J Pharm Biomed Anal. 2009;50(4):664-70.

8 European Medicinces Agency. Afinitor, Highlights of prescribing information. 2009. Retrieved from https://www.accessdata.fda. gov/drugsatfda_docs/label/2012/022334s016l bl.pdf. Accessed May 2017.

9 de Wit D, Schneider TC, Moes DJ,et al. Everolimus pharmacokinetics and its exposure-toxicity relationship in patients with thyroid cancer. Cancer Chemother Pharmacol. 2016;78(1):63-71.

10 Deppenweiler M, Falkowski S, Saint-Marcoux $F$, et al. Towards therapeutic drug monitoring of everolimus in cancer? Results of an exploratory study of exposure-effect relationship. Pharmacol Res. 2017;121:138-44.

11 Ravaud A, Urva SR, Grosch K, et al. Relationship between everolimus exposure and safety and efficacy: meta-analysis of clinical trials in oncology. Eur J Cancer. 2014;50(3):486-95.

12 Thiery-Vuillemin A, Mouillet G, Nguyen Tan Hon $\mathrm{T}$, et al. Impact of everolimus blood concentration on its anti-cancer activity in patients with metastatic renal cell carcinoma. Cancer Chemother Pharmacol. 2014;73 (5):999-1007.

13 US Food \& Drug Administration. Drug Approval Package. Afinitor. 2009. Retrieved from https://www.accessdata.fda.gov/ drugsatfda_docs/nda/2009/022334s000TOC.c fm. Accessed February 2017.

14 O'Donnell A, Faivre S, Burris HA 3rd, et al. Phase I pharmacokinetic and pharmacodynamic study of the oral mammalian target of rapamycin inhibitor everolimus in patients with advanced solid tumors. J Clin Oncol. 2008;26(10):1588-95.

15 Awada A, Cardoso F, Fontaine C, et al. The oral mTOR inhibitor RAD001 (everolimus) in combination with letrozole in patients with advanced breast cancer: results of a phase I study with pharmacokinetics. Eur J Cancer. 2008;44(1):84-91.

16 de Wit D, den Hartigh J, Gelderblom $\mathrm{H}$, et al. Dried blood spot analysis for therapeutic drug monitoring of pazopanib. J Clin Pharmacol. 2015;55(12):1344-50.

17 Kralj E, Trontelj J, Pajic T, et al. Simultaneous measurement of imatinib, nilotinib and dasatinib in dried blood spot by ultra high performance liquid chromatography tandem mass spectrometry. J Chromatogr B Analyt Technol Biomed Life Sci. 2012;903:150-6.

18 Antunes MV, Raymundo S, Wagner SC, et al. DBS sampling in imatinib therapeutic drug monitoring: from method development to clinical application. Bioanalysis. 2015;7 (16):2105-17.

19 Nijenhuis CM, Huitema AD, Marchetti S, et al. The Use of Dried Blood Spots for Pharmacokinetic Monitoring of Vemurafenib Treatment in Melanoma Patients. J Clin Pharmacol. 2016;56(10):1307-12. 
20 Koster RA, Veenhof $\mathrm{H}$, Botma R, et al. Dried blood spot validation of five immunosuppressants, without hematocrit correction, on two LC-MS/MS systems. Bioanalysis. 2017;9(7):553-63.

21 Koster RA, Alffenaar JW, Greijdanus B, et al. Fast LC-MS/MS analysis of tacrolimus, sirolimus, everolimus and cyclosporin $A$ in dried blood spots and the influence of the hematocrit and immunosuppressant concentration on recovery. Talanta. 2013;115: 47-54.

22 Wang $\mathrm{Y}$, Chia $\mathrm{YL}$, Nedelman J, et al. A therapeutic drug monitoring algorithm for refining the imatinib trough level obtained at different sampling times. Ther Drug Monit. 2009;31(5):579-84.

23 Knapen LM, Beer Yd, Brüggemann RJM, et al. Development and validation of an analytical method using UPLC-MS/MS to quantify everolimus in dried blood spots in the oncology setting. J Pharm Biomed Anal. 2018; 149:106-13.

24 Clinical and Laboratory Standards Institute. 2013. Measurement Procedure Comparison and Bias Estimation Using Patient Samples; Approved Guideline-Third Edition.

25 MEDCALC. Easy-to-use statistical software. Retrieved from https://www.medcalc.org/ manual/sampling_blandaltman.php. Accessed February 2017.

26 Bland JM, Altman DG. Statistical methods for assessing agreement between two methods of clinical measurement. Lancet. 1986;1 (8476):307-10.

27 Passing $\mathrm{H}$, Bablok. A new biometrical procedure for testing the equality of measurements from two different analytical methods. Application of linear regression procedures for method comparison studies in clinical chemistry, Part I. J Clin Chem Clin Biochem. 1983;21(11):709-20.

28 Sheiner LB, Beal SL. Some suggestions for measuring predictive performance. J Pharmacokinet Biopharm. 1981;9(4):503-12.

29 European Medicines Agency. Guideline on bioanalytical method validation. 21 July 2011. Retrieved from https://www.ema.europa.eu/ documents/scientific-guideline/guidelinebioanalytical-method-validation_en.pdf. Accessed March 2017.

30 van Amsterdam $\mathrm{P}$, Companjen A, BrudnyKloeppel $M$, et al. The European Bioanalysis
Forum community's evaluation, interpretation and implementation of the European Medicines Agency guideline on Bioanalytical Method Validation. Bioanalysis. 2013;5 (6):645-59.

31 van Erp NP, van Herpen CM, de Wit D, et al. A Semi-Physiological Population Model to Quantify the Effect of Hematocrit on Everolimus Pharmacokinetics and Pharmacodynamics in Cancer Patients. Clin Pharmacokinet. 2016;55(11):1447-56.

32 US Food \& Drug Administration. Center for Drug Evaluation and Research. Application Number: 22-334. Clinical Pharmacology and Biopharmaceutics Review(s). Afinitor. 2008. Retrieved from https://www.accessdata.fda. gov/drugsatfda_docs/nda/2009/022334s000_ ClinPharmR.pdf. Accessed March 2017.

33 de Vries R, Barfield M, van de Merbel N, et al. The effect of hematocrit on bioanalysis of DBS: results from the EBF DBS-microsampling consortium. Bioanalysis. 2013;(17):2147-60.

34 Koster RA, Botma R, Greijdanus B, et al. The performance of five different dried blood spot cards for the analysis of six immunosuppressants. Bioanalysis. 2015;7(10):122535.

35 Kip AE, Kiers KC, Rosing $H$, et al. Volumetric absorptive microsampling (VAMS) as an alternative to conventional dried blood spots in the quantification of miltefosine in dried blood samples. J Pharm Biomed Anal. 2017;135:160-6.

36 Jager NG, Rosing $\mathrm{H}$, Linn SC, et al. Dried Blood Spot Self-Sampling at Home for the Individualization of Tamoxifen Treatment: A Feasibility Study. Ther Drug Monit. 2015;37 (6):833-6.

37 Cheung $\mathrm{CY}$, van der Heijden J, Hoogtanders $\mathrm{K}$, et al. Dried blood spot measurement: application in tacrolimus monitoring using limited sampling strategy and abbreviated AUC estimation. Transpl Int. 2008;21(2):140-5.

38 Kromdijk W, Mulder JW, Smit PM, et al. Therapeutic drug monitoring of antiretroviral drugs at home using dried blood spots: a proof-of-concept study. Antivir Ther. 2013;18 (6):821-5.

39 Boons C, Chahbouni A, Schimmel AM, et al. Dried blood spot sampling of nilotinib in patients with chronic myeloid leukaemia: a comparison with venous blood sampling. J Pharm Pharmacol. 2017;69(10):1265-74. 


\section{Chapter 4.3}

Plasma concentrations of tyrosine kinase inhibitors imatinib, erlotinib, and sunitinib in routine clinical outpatient cancer care

N.A.G. Lankheet, L.M. Knapen, J.H.M. Schellens, J.H. Beijnen, N. Steeghs,

A.D.R. Huitema

Ther Drug Monit. 2014;36(3):326-34 


\section{Abstract}

\section{Background / objectives}

The objectives of this study were to evaluate the plasma concentrations of the tyrosine kinase inhibitors (TKIs), imatinib, erlotinib, and sunitinib, in a cohort of patients with cancer in routine clinical practice and to find the possible factors related to plasma concentrations below the target level.

\section{Patients and methods}

An observational study was performed in an unselected cohort of patients using TKIs for cancer treatment. Randomly timed plasma samples were drawn together with regular laboratory investigations during routine outpatient clinic visits. The plasma concentrations of TKIs were determined using a validated high-performance liquid chromatography coupled with tandem mass spectrometry detection method. Trough concentrations were estimated using the interval between the last dose intake and blood sampling and the mean elimination half-life of the TKIs and were compared with target trough concentrations. Outpatient medical records were reviewed to collect data on patient- and medication-related factors that could have contributed to the variation in TKI plasma concentrations.

\section{Results}

Only $26.8 \%, 88.9 \%$ and $51.4 \%$ of the calculated trough plasma concentrations of imatinib, erlotinib, and sunitinib samples, respectively, reached the predefined target concentration (imatinib: $1100 \mathrm{ng} / \mathrm{mL}$, erlotinib: $500 \mathrm{ng} / \mathrm{mL}$, and sunitinib: $50 \mathrm{ng} / \mathrm{mL}$ ). Interpatient variability was high with coefficients of variation of $39.1 \%, 40.1 \%$, and $29.2 \%$ for imatinib, erlotinib, and sunitinib, respectively. High variation in plasma concentrations could only partly be explained by patient- or medication-related factors.

\section{Conclusions}

Almost half of the plasma concentrations in the outpatient population seemed to be below the target level with a risk of treatment failure. It is not possible to predict which patients are at a risk of plasma concentrations below the target level based on patient- or medication-related factors. Thus, therapeutic drug monitoring could play a crucial role in routine cancer care to identify patients that are in need of individual adjusted dosages. Further research is required to investigate the safety and efficacy of therapeutic drug monitoring. 


\section{Introduction}

Tyrosine kinase inhibitors (TKIs) are targeted agents, which have been introduced in anticancer treatment within the last 2 decades and became an integral part of this treatment rapidly. Application of these agents in patients with malignant diseases has shown to successfully induce clinical responses in various malignancies ${ }^{1}$.

For several TKIs, relationships existing between plasma concentrations and treatment efficacy and toxicity have been described ${ }^{2-8}$. Pharmacokinetic variability (both interpatient and intrapatient) may, therefore, have important clinical consequences. For imatinib, erlotinib, and sunitinib, minimal plasma concentration thresholds have been established below which a substantial increase in treatment failure and drug resistance was observed ${ }^{5,7,9-15}$.

The ease of oral administration of TKIs enables patients to get their drug regimen in an outpatient setting, which is more patient friendly. However, oral administration also entails the possibility of considerable variation in drug exposure due to patient nonadherence (e.g. due to drug-related toxicity), drug interactions with co-medication, and variability in oral drug availability ${ }^{16}$. Although pharmacokinetic parameters, such as metabolic clearance and bioavailability, can vary substantially among individuals and especially during illness ${ }^{17}$, TKIs are currently prescribed at a fixed dose. The large interindividual variability in systemic exposure in combination with the positive exposure-efficacy relationship and low therapeutic index of TKIs, form a rationale for therapeutic drug monitoring (TDM) of these drugs ${ }^{10,18-21}$.

We have investigated the occurrence of TKI plasma concentrations below the target level in an outpatient population. In addition, we have studied patient-related (e.g. gender, age, and weight) and medication-related (e.g. dose and drug interactions with concomitantly administered drugs) factors that may contribute to the occurrence of TKI concentrations below the target level. The data may unveil some valuable arguments about the role of TDM in an unselected cohort of patients in routine daily clinical practice. Further, the outcome may support further optimisation and individualisation of TKI therapy.

\section{Methods}

\section{Patients}

Patients were included if a TKI was part of their anticancer therapy in the period from June 2009 to May 2012. The patients were willing and able to undergo blood sampling. No exclusion criteria were defined, because the cohort of patients should reflect an unselected, 'real-life' cohort of patients. For a patient to be evaluable, at least one drug plasma concentration during the steady state should be available during the defined 
period. The study protocol was approved by the local independent ethics committees. The patients received information regarding the purpose and conduct of this study and provided written informed consent. A few plasma samples included in the analysis were drawn on a request from the physician for TDM purposes and to substantiate clinical observations as part of the treatment follow-up.

Patients using TKIs other than imatinib, erlotinib, and sunitinib were excluded from the analysis, because patient numbers were too small. All plasma samples were single randomly timed samples obtained by standardised procedures using a venapuncture. The samples were drawn together with regular laboratory investigations during routine outpatient clinic visits. Time after drug administration (interval between last dose intake and plasma sampling) and dosing scheme were documented. Patients whose dosing scheme or the time interval after the last intake was not available were excluded from the final analysis.

\section{Bioanalysis}

Ethylenediaminetetraacetic acid whole-blood samples were centrifuged within 36 hours of collection without stability issues. Subsequently, ethylenediaminetetraacetic acid plasma was stored at $-70^{\circ} \mathrm{C}$ until analysis. Plasma concentrations of imatinib, erlotinib, sunitinib, and its active metabolite $\mathrm{N}$-desethyl sunitinib were determined by validated high-performance liquid chromatography coupled with tandem mass spectrometry detection methods ${ }^{22,23}$. Lower limits of quantification were $20 \mathrm{ng} / \mathrm{mL}$ for imatinib and erlotinib. For sunitinib and its metabolite, the lower limits of quantification were $5 \mathrm{ng} / \mathrm{mL}$.

\section{Trough plasma concentrations}

For each TKI, trough target plasma concentrations that need to be reached for effective therapy were defined based on published data and are shown in Table 4.3.1. For each patient sample, trough plasma concentrations were compared with the target trough concentrations. Blood samples were drawn during follow-up visits at the outpatient clinic along with routine laboratory tests. Most patients took their medication early in the morning or late in the evening. Therefore, the plasma samples were randomly drawn during the dosing interval within this study and trough plasma concentrations were not always available. Therefore, trough plasma concentrations were estimated using the interval between last dose intake and blood sampling and the mean elimination half-life of the drugs, as previously proposed by Wang et al. ${ }^{24}$. The formulas used for this purpose were:

1) Conc $_{24 h}=$ Conc $_{\text {measured }} \times 0.5\left(\frac{24-\text { interval }}{t^{\prime / 2}}\right)$

2) Conc $_{12 h}=$ Conc $_{\text {measured }} \times 0.5\left(\frac{24-\text { interval }}{t^{1 / 2}}\right)$ 
Here, Conc $_{24 \mathrm{~h}}$ and $\mathrm{Conc}_{12 \mathrm{~h}}$ are the calculated plasma trough levels for drugs with once daily or twice daily administration, respectively; Conc $_{\text {measured }}$ is the measured drug plasma concentration in nanograms per millilitre; interval is the interval between the last dose intake and blood sampling in hours; $\mathrm{t}^{1 / 2}$ is the mean terminal half-life of the drug in hours.

Formula 1 was used for drugs with once daily administration, and formula 2 was used for drugs with twice daily administration. Mean elimination half-lives used in the calculation were 18,36, 50, and 95 hours for imatinib, erlotinib, sunitinib, and $N$-desethyl sunitinib, respectively ${ }^{10,19,20}$. Subsequently, estimated trough concentrations were categorised as below or above the target trough level. A linear mixed effects model was used to assess interpatient and intrapatient variabilities in trough concentrations.

To calculate dose-corrected plasma concentrations, the most frequently prescribed mean daily doses within our cohort, namely, $400 \mathrm{mg}$ for imatinib, $150 \mathrm{mg}$ for erlotinib, and $50 \mathrm{mg}$ for sunitinib were used as standard mean daily doses.

Table 4.3.1 Target trough plasma concentrations of tyrosine kinase inhibitors.

\begin{tabular}{lccc}
\hline Drug & Target trough level $(\mathrm{ng} / \mathrm{mL})$ & Subgroup & References \\
\hline Imatinib & 1000 & $\mathrm{CML}^{\mathrm{a}}$ & 7,15 \\
Imatinib & 1110 & $\mathrm{GIST}^{\mathrm{a}}$ & 10 \\
Erlotinib & 500 & $\mathrm{~b}$ & 5 \\
Suntinib & $50-100$ & $9,11-14$ \\
\hline
\end{tabular}

CML: chronic myeloid leukemia; GIST: gastrointestinal stromal tumour. ${ }^{a}$ Based on preclinical pharmacokinetic-pharmacodynamic data. ${ }^{b}$ sum of sunitinib and $N$-desethyl sunitinib level.

\section{Patient data collection}

To study patient- and medication-related factors that could have contributed to variations in TKI plasma concentrations, outpatient medical records were reviewed. Data were collected on the daily dose, age, gender, weight, liver function tests [aspartate aminotransferase (AST), alanine aminotransferase (ALT)], cigarette smoking behaviour, tumour type, and concomitantly administered drugs.

\section{Toxicity and plasma concentrations}

The causality relation of reported adverse events with TKI use was determined on the investigator's discretion using 2 criteria: (1) Are there previous conclusive reports on this reaction and (2) Did the adverse event occur after the TKI was administered ${ }^{25}$. Data on the occurrence of adverse events, which were clinically relevant and possibly or probably related to TKI use, were extracted from medical records. It was assessed which TKI plasma concentrations were related to the occurrence of toxicity. Subsequently, the prevalence of adverse events in patients with plasma concentrations below or above the target level was investigated. 


\section{Factors influencing plasma concentrations}

To assess the correlation between all patient- and medication-related factors (except daily dose) and TKI plasma concentrations, dose-corrected plasma concentrations were used as dose-exposure relationships were found to be linear ${ }^{11,26,27}$. The correlation between patient-related factors age, gender, body weight, or liver function and TKI plasma concentrations was assessed. Additionally, 4 medication-related factors were investigated. First, the correlation between the mean daily TKI dose and TKI plasma concentrations was determined. Second, the influence of concomitant medication, which induces or inhibits cytochrome P450 enzyme 3A4 (CYP3A4), was assessed, because TKIs are mainly metabolised via CYP3A4 ${ }^{10,19,20}$. Third, the bioavailability of erlotinib is known to depend on gastric $\mathrm{pH}<5.0$ and the bioavailability of imatinib is known to depend on drug efflux transporters [P-glycoprotein (P-gp) and breast cancer resistance protein (BCRP)]. Therefore, the impact of the use of $\mathrm{H}_{2}$-antagonists and proton pump inhibitors (PPIs), which increase gastric $\mathrm{pH}$ and inhibit $\mathrm{P}$-gp and BCRP ${ }^{28-31}$, on TKI plasma concentrations was investigated. At last, the effect of cigarette smoking on erlotinib concentrations was investigated, because smoking is known to decrease erlotinib exposure by induction of CYP $1 \mathrm{~A}^{27}$. For all patient- and medication-related factors, correlations with plasma concentrations were tested using linear mixed effect modeling (NONMEM 7; Icon Development Solutions, Ellicott City, MD, 2009). The likelihood ratio test was used to assess the significance of different parameters (patient- and medication-related factors) in the regression models. A p value of $<0.05$ was considered statistically significant ${ }^{32}$.

Additionally, for each plasma concentration that was classified as within approximately the lowest 10th percentile of concentrations, it was investigated whether 1 of the above mentioned factors could be identified as the cause of the very low plasma concentrations. Although no upper limit of the therapeutic window of plasma trough levels has been defined for TKIs, extremely high TKI plasma concentrations were also investigated in a similar manner. Therefore, the involvement of possible patient- and medication-related factors was investigated for the approximately $5 \%$ highest plasma concentrations of each TKI.

\section{Results}

\section{Patients and plasma concentrations}

Finally, 108 evaluable patients were included in the study, with a mean age of 61 years (range 33-83 years). More than half of the patients were female (58\%). A total of 246 plasma concentrations were available, yielding a median of 2 plasma concentrations per patient (range 1-7). Characteristics of all the patients enrolled are depicted in Table 4.3.2 stratified by TKI therapy. 
Table 4.3.2 Characteristics of patients on imatinib, erlotinib, and sunitinib therapy.

\begin{tabular}{|c|c|c|c|c|c|c|}
\hline \multirow{2}{*}{$\begin{array}{l}\text { Characteristic } \\
\text { Number of patients }\end{array}$} & \multicolumn{2}{|c|}{ Imatinib } & \multicolumn{2}{|c|}{ Erlotinib } & \multicolumn{2}{|c|}{ Sunitinib } \\
\hline & & 36 & & 41 & & 31 \\
\hline Number of samples & & 112 & & 99 & & 35 \\
\hline \multicolumn{7}{|l|}{$\begin{array}{l}\text { Samples per patient } \\
\text { (no. of patients, \%) }\end{array}$} \\
\hline 1 & & $9(25.0)$ & & $10(24.4)$ & & $27(87.1)$ \\
\hline 2 & & 5 (13.9) & & $16(39.0)$ & & 4 (12.9) \\
\hline 3 & & $9(25.0)$ & & 6 (14.6) & & \\
\hline 4 & & 5 (13.9) & & $7(17.1)$ & & \\
\hline 5 & & $4(11.1)$ & & $1(2.4)$ & & \\
\hline 6 & & $2(5.6)$ & & $1(2.4)$ & & \\
\hline 7 & & $2(5.6)$ & & & & \\
\hline \multicolumn{7}{|l|}{$\begin{array}{l}\text { Gender (no. of } \\
\text { patients, \%) }\end{array}$} \\
\hline Male & & $14(38.9)$ & & 15 (36.6) & & $16(51.6)$ \\
\hline Female & & $22(61.1)$ & & $26(63.4)$ & & $15(48.4)$ \\
\hline Mean age (years, SD) & & $60(11.1)$ & & $60(7.9)$ & & $63(9.9)$ \\
\hline $\begin{array}{l}\text { Mean body weight (kg, } \\
\text { SD) }\end{array}$ & & $78(18.7)$ & & $75(15.3)$ & & $83(17.8)$ \\
\hline \multicolumn{7}{|l|}{$\begin{array}{l}\text { SD) } \\
\text { Tumour type } \\
\text { (no. of patients, \%) }\end{array}$} \\
\hline & GIST & $35(97.2)$ & NSCLC & 41 (100) & $\mathrm{mRCC}$ & $29(93.5)$ \\
\hline & $\mathrm{CML}$ & $1(2.8)$ & & & GIST & $2(6.5)$ \\
\hline \multicolumn{7}{|l|}{$\begin{array}{l}\text { Dosing scheme } \\
\text { (no. of patients, \%) }\end{array}$} \\
\hline & 200 mg QD & $2(5.6)$ & $25 \mathrm{mg} Q \mathrm{D}$ & $1(2.4)$ & $25 \mathrm{mg} \mathrm{QD}^{\mathrm{a}}$ & $2(6.5)$ \\
\hline & $300 \mathrm{mg}$ QD & $1(2.8)$ & $50 \mathrm{mg}$ QD & $2(4.9)$ & $37.5 \mathrm{mg} \mathrm{QD}{ }^{\mathrm{a}}$ & $12(38.7)$ \\
\hline & $400 \mathrm{mg} Q D$ & $31(86.0)$ & $75 \mathrm{mg}$ QD & $2(4.9)$ & $50 \mathrm{mg} \mathrm{QD}^{\mathrm{a}}$ & $15(48.4)$ \\
\hline & 300 mg BID & $1(2.8)$ & $100 \mathrm{mg}$ QD & $3(7.3)$ & $62.5 \mathrm{mg} \mathrm{QD}{ }^{\mathrm{a}}$ & $2(6.5)$ \\
\hline & 400 mg BID & $1(2.8)$ & $150 \mathrm{mg}$ QD & $32(78.1)$ & & \\
\hline & & & $100 \mathrm{mg}$ eod & $1(2.4)$ & & \\
\hline $\begin{array}{l}\text { Median sampling time } \\
\text { (hours, range) }\end{array}$ & & $15,4(3-24)$ & & $12,0(3-44)$ & & $14,3(2-44)$ \\
\hline
\end{tabular}

BID: twice daily; CML: chronic myeloid leukemia; eod: every other day; GIST: gastrointestinal stromal tumour; mRCC: metastasised renal cell carcinoma; no; number: NSCLC: non-small cell lung cancer; QD: once daily; SD: standard deviation. ${ }^{a}$ Patients were treated with sunitinib for 4 weeks followed by 2 weeks off-treatment. Plasma samples were analysed during steady state of the on-treatment phase.

The mean trough plasma concentrations for imatinib, erlotinib, and the sum of sunitinib and $N$-desethyl sunitinib were $926 \mathrm{ng} / \mathrm{mL}$ (range $87.0-3607$ ), $1010 \mathrm{ng} / \mathrm{mL}$ (range 74.9-5542) and $51.6 \mathrm{ng} / \mathrm{mL}$ (range 14.7-93.7), respectively. In the total study population, target trough plasma concentrations were reached in $55.3 \%$ of patient samples. For the particular patient groups using imatinib, erlotinib, or sunitinib treatment, target trough concentrations were reached in $26.8 \%, 88.9 \%$, and $51.4 \%$ of patient samples, respectively. The distribution of trough concentrations divided into below or above the target plasma concentrations is depicted in Table 4.3.3. 
Table 4.3.3 Distribution of (calculated) trough concentrations divided in below and above target levels.

\begin{tabular}{lcrcrcr}
\hline TKI & \multicolumn{2}{c}{ Total } & \multicolumn{2}{c}{ Below target level } & \multicolumn{2}{c}{ Above target level } \\
\cline { 2 - 6 } & Number & Mean (CV\%) & Number (\%) & Mean (CV\%) & Number (\%) & Mean (CV\%) \\
\hline Imatinib & 112 & $926(51.7)$ & $82(73.2)$ & $712(32.0)$ & $30(26.8)$ & $1508(33.4)$ \\
Erlotinib & 99 & $1011(68.6)$ & $11(11.1)$ & $288(57.9)$ & $88(88.9)$ & $1101(61.9)$ \\
Sunitinib & 35 & $51.6(38.7)$ & $17(48.6)$ & $35.5(26.5)$ & $18(51.4)$ & $68.6(18.2)$ \\
Total & 246 & & $110(44.7)$ & & $136(55.3)$ & \\
\hline
\end{tabular}

CV\%: coefficient of variation; mean: mean plasma concentration in nanograms per millilitre; TKI: tyrosine kinase inhibitor.

A large variability in the trough concentrations reached was observed for all drugs, as shown in Figure 4.3.1. Interpatient variability in plasma concentrations with coefficient of variation (CV\%) of $39.1 \%, 40.1 \%$, and $29.2 \%$ were found for imatinib, erlotinib, and sunitinib, respectively. From 26 patients on imatinib therapy, $>1$ plasma sample was available, and the intrapatient variability of plasma concentrations in this group was $32.9 \%$ (CV\%). For erlotinib, the intrapatient variability, based on 30 patients with multiple plasma samples, was $35.5 \%$ (CV\%). Only 4 patients with multiple plasma samples were included in the sunitinib group, and the intrapatient variability of total plasma concentrations in those patients was $34.5 \%$ (CV\%).

\section{Trough levels in relation to toxicities}

During the study period, 66 patients (61.1\%) suffered from toxicities: 19 patients (52.8\%) on imatinib therapy, 25 patients (61.0\%) on erlotinib therapy, and 22 patients (71.0\%) on sunitinib therapy. The incidence of reported adverse events is shown in Table 4.3.4. Consequently, 4 patient groups could be defined, namely, (1) patients with drug concentrations below the target level and without toxicities; (2) patients with drug concentrations below the target level and with toxicities; (3) patients with drug levels above the target level and without toxicities; and (4) patients with drug levels above the target level and with toxicities. The distribution of patient plasma samples within these 4 groups is depicted in Figure 4.3.2. No difference was observed between the mean trough plasma concentrations of patients with or without toxicities, as shown in Figure 4.3.3. Toxicities also occurred at drug concentrations below the target level. For sunitinib and erlotinib, the prevalence of toxicities in patients with plasma concentrations above the target level was higher than in patients with plasma concentrations below the target levels. 

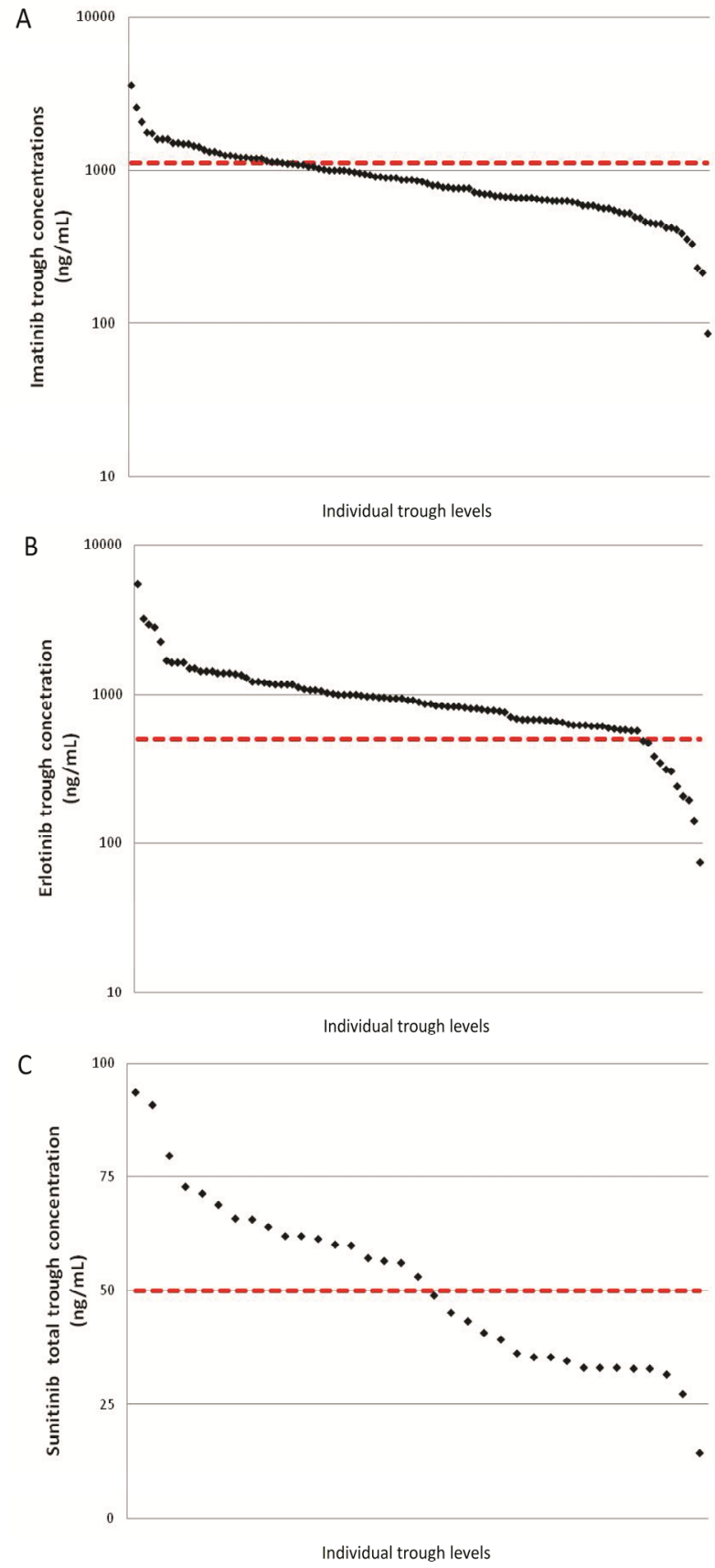

Figure 4.3.1 Calculated trough plasma concentrations for all plasma samples of imatinib (A), erlotinib (B), and sunitinib (C) patients. The dashed line represents the target level below which trough plasma concentrations were classified as below the target level. 
Table 4.3.4 Incidence of toxicity.

\begin{tabular}{|c|c|c|c|c|c|c|}
\hline \multirow{2}{*}{$\begin{array}{l}\text { Characteristic } \\
\text { Patients with toxicity } \\
\text { (no. of patients, \%) }\end{array}$} & \multicolumn{2}{|l|}{ Imatinib } & \multicolumn{2}{|c|}{ Erlotinib } & \multicolumn{2}{|c|}{ Sunitinib } \\
\hline & & $19(52.8)$ & & $25(61.0)$ & & $22(71.0)$ \\
\hline $\begin{array}{l}\text { Samples related to } \\
\text { toxicity (no. of } \\
\text { patients, \%) }\end{array}$ & & $61(54.4)$ & & $64(64.6)$ & & $23(65.7)$ \\
\hline \multirow{12}{*}{$\begin{array}{l}\text { Toxicity (no. of } \\
\text { patients, \%) }\end{array}$} & Fatigue & $6(16.7)$ & Skin rash & $22(53.7)$ & Fatigue & $9(29.0)$ \\
\hline & $\begin{array}{l}\text { Periorbital } \\
\text { edema }\end{array}$ & $6(16.7)$ & Diarrhea & $6(14.6)$ & $\begin{array}{l}\text { Hand foot } \\
\text { syndrome }\end{array}$ & $7(22.6)$ \\
\hline & Nausea & $4(11.1)$ & Blepharitis & $4(9.8)$ & Mucositis & $7(22.6)$ \\
\hline & Skin rash & $3(8.3)$ & Paronychia & $4(9.8)$ & Dysgeusia & 4 (12.9) \\
\hline & Diarrhea & $2(5.6)$ & Vomiting & $2(4.9)$ & Stomatitis & $3(9.7)$ \\
\hline & Dyspnea & $2(5.6)$ & Fatigue & $2(4.9)$ & Hypertension & $2(6.5)$ \\
\hline & Peripheral edema & $2(5.6)$ & Anorexia & $2(4.9)$ & Anorexia & $2(6.5)$ \\
\hline & Dysgeusia & $1(2.8)$ & Weight loss & $1(2.4)$ & Dyspepsia & $2(6.5)$ \\
\hline & Hypertension & $1(2.8)$ & Dysgeusia & $1(2.4)$ & Vomiting & $1(3.2)$ \\
\hline & Alopecia & $1(2.8)$ & Nausea & $1(2.4)$ & Skin rash & $1(3.2)$ \\
\hline & Anorexia & $1(2.8)$ & & & Muscle cramps & $1(3.2)$ \\
\hline & Anemia & $1(2.8)$ & & & Hypothyroidism & $1(3.2)$ \\
\hline
\end{tabular}

No: number.

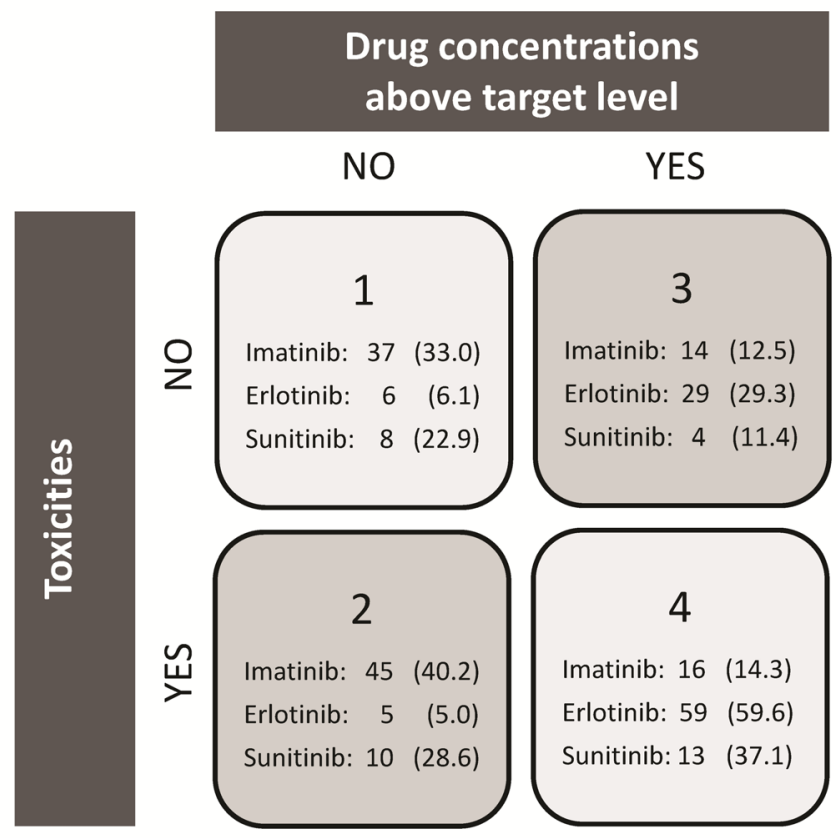

Figure 4.3.2 Four subgroups of patients samples can be defined based on whether or not reaching target drug concentrations and whether or not related to occurrence of toxicities. Distribution of patient samples within these 4 groups is depicted with number and percentage (within parentheses) of samples for each tyrosine kinase inhibitor. 


\section{Factors influencing plasma concentrations}

Our cohort comprised patients with a large range of body weights. Despite obesity, all patients with extremely high body weights were treated with standard doses of TKIs. However, no significant effect of body weight on TKI plasma concentrations was observed. No large differences were found in plasma concentrations between different age groups. Additionally, no significant effects of gender on plasma concentrations were observed for erlotinib and sunitinib. For imatinib, a trend of higher plasma concentrations in females was observed $(+39 \%, p>0.05)$. Slight increases of plasma concentrations were observed for imatinib $(+24 \%, p>0.05)$ and erlotinib $(+23 \%, p>0.05)$ with decreasing liver function. For erlotinib concentrations, a dose-exposure correlation was shown, but due to large interpatient variability, no linear relationship was found. For imatinib and sunitinib, no relationship between dose and plasma concentrations was observed in our patient cohort. The effect of PPIs on erlotinib and imatinib plasma concentrations was not significant $[-27.8 \%(p>0.05)$ and $+8.0 \%(p>0.05)$, respectively]. A significant increase of sunitinib concentrations $(+53 \%, p<0.05)$ was observed with concomitant use of PPIs. Eleven plasma samples of two patients, using imatinib concomitantly with CYP3A4 inducers (carbamazepine or phenytoin), showed significantly decreased imatinib plasma concentrations $(-58.4 \%, p<0.05)$. In one of the patients, a dose increase from $400 \mathrm{mg}$ once daily to subsequently 300 and $400 \mathrm{mg}$ twice daily was implemented. However, even when comparing measured plasma concentrations instead of dose-corrected plasma concentrations, the imatinib concentrations were significantly decreased $(-51.1 \%, p<0.05)$. Five erlotinib concentrations of cigarette smokers were measured, and the CYP1A enzyme inducing effect of smoking was correlated to an insignificant decrease of erlotinib concentrations $(-37.0 \%, p>0.05)$.

Only $11(11.1 \%)$ of the erlotinib samples were below the target level of $500 \mathrm{ng} / \mathrm{mL}$. Among these samples were two samples of heavy cigarette smokers; three samples of patients with low erlotinib doses ( $25 \mathrm{mg}$ QD or $150 \mathrm{mg}$ every other day); five samples of patients using medication that increases the gastric $\mathrm{pH}$; and one sample for which no explanation could be found for plasma concentrations below the target level. Four sunitinib samples (11.4\%) were below a total sum concentration of $30 \mathrm{ng} / \mathrm{mL}$. The extremely low concentration in one of the samples could possibly be explained by a high body weight of $125 \mathrm{~kg}$. Potential causes for the other low sunitinib concentrations were not identified. Fifteen imatinib samples (13.4\%) obtained from eight different patients were $<500 \mathrm{ng} / \mathrm{mL}$. Seven of those extremely low plasma concentrations were attributed to the drug-drug interactions with CYP3A4-inducing agents, as mentioned above. For the other eight samples, no patient- or medication-related factor could be identified to explain the trough plasma concentrations below the target level.

In three patient samples $(2.7 \%)$, very high trough concentrations of imatinib $(\geq 2000 \mathrm{ng} / \mathrm{mL}$ ) were found. For two of these samples, no explanation was found for the 
extremely high concentrations. The third sample was of a patient treated with $300 \mathrm{mg}$ imatinib twice daily. In two samples (5.7\%), high sunitinib plasma concentrations $(>90 \mathrm{ng} / \mathrm{mL}$ ) were measured. One of the patients had increased AST and ALT activities in the serum. For erlotinib, the highest observed plasma concentrations could possibly be explained by hepatic dysfunction. Of the five patients (5.1\%) with the highest plasma concentrations ( $\geq 2000 \mathrm{ng} / \mathrm{mL}$ ), all patients had increased ALT and AST activities in serum.

\section{Discussion}

The objective of this study was to explore the distribution of trough plasma concentrations of imatinib, erlotinib, and sunitinib in an unselected cohort of patients. Only 55.3\% of the trough plasma concentrations reached the predefined target level. In our cohort, the large interpatient variability of plasma concentrations could only be explained partly by patient- or medication-related factors. This implicates that routine TDM may be very valuable for treatment optimisation and individualisation of these drugs.

As far as we know, this is the first investigation of trough plasma concentrations of various TKIs in an unselected population. Imatinib trough concentrations have been determined previously in different cohorts of patients who were enrolled in clinical trials with strict inclusion and exclusion criteria (e.g. patients with poor compliance to treatment or using concomitant medication were excluded) $)^{7,14,15}$. For imatinib, additionally, some observational studies without exclusion criteria have been performed in which the influence of patient-related factors on plasma trough levels was investigated $^{8,33,34}$.

We used an unselected cohort of patients to approach the outpatient cancer patient care as best as possible. In such 'real-life' cohort, variability in drug exposure may be much larger than in clinical trials, and consequently, response to treatment (efficacy and/or toxicity) may show a wider variability. With this observational study design, it is, however, difficult to detect factors that significantly affect plasma concentrations. Another drawback of the observational study design is that only clinically relevant toxicity was recorded and, thus, toxicity was not systematically graded according to the common terminology criteria for adverse events. However, because the main goal of the study was to explore the range of trough plasma concentrations that was reached in a 'real-life' patient population, it was accepted that only exploratory investigations would be achievable on possible determinants of variation in plasma concentrations and adverse events. In addition, patients with a wide variability of tumour types, disease stages, pretreatment and treatment duration were included in our study. For this reason, it was not justified to compare clinical outcomes, as, for example, biomarkers for molecular response in chronic myeloid leukemia (CML), 
during imatinib therapy, between patients with plasma concentrations below and above the target level.

The algorithm to estimate trough plasma concentrations was derived from the method to estimate imatinib trough plasma concentrations previously described by Wang et al. ${ }^{24}$. This method using population elimination rate was feasible to calculate the trough level of individual plasma samples obtained at different time points in the elimination phase of the drug with acceptable deviations from real trough levels. Because the elimination half-lives of erlotinib and sunitinib are even longer than the elimination half-life of imatinib, the use of a similar algorithm to estimate trough plasma concentrations was justified. The algorithm was validated for samples drawn \pm 7 hours around the 24 hour postdose time point. Using this algorithm with plasma samples drawn within the absorption phase of the drug (2-4 hours postdose for imatinib, 1-7 hours for erlotinib and 6-12 hours sunitinib), one could underestimate the trough plasma concentration. For most patients in our study, the samples were collected well after the time to reach the maximum plasma concentration $\left(T_{\text {max }}\right)^{35}$. However, in a few cases, the samples were collected somewhat earlier. As this study is exploratory and the major aim was to assess the variability in concentrations in a 'real-life' cohort of patients, we have included these patients in the final analysis. However, if TDM is to be implemented, dosing advices based on concentrations collected before or around the $T_{\max }$ should be avoided. For sunitinib, the active metabolite was incorporated in the target trough plasma concentration that was described in published data. Therefore, besides sunitinib also the active metabolite $\mathrm{N}$-desethyl sunitinib was analysed to determine the total trough plasma concentration. For imatinib and erlotinib, target plasma concentrations were based solely on the parent compounds. Therefore, the metabolites were not included in the analysis of imatinib and erlotinib trough plasma concentrations.

High percentages of samples were below the target level for imatinib and sunitinib, namely, $73.2 \%$, and $48.6 \%$, respectively. Because multiple samples per patient were analysed, these percentages do not directly reflect the number of patients that are on suboptimal regimen. Nevertheless, our results are comparable with the percentage of patients reaching subtarget concentrations of imatinib and sunitinib in previously reported studies ${ }^{7,14,34,36}$. The relatively high number of imatinib concentrations below the target level can probably be explained by decreasing imatinib exposure during eight months after the start of the treatment, for example, due to the induction of hepatic enzymes, as reported by Eechoute et al. ${ }^{37}$, because most patients included in our study had already been treated with imatinib for several years. Although our patient cohort comprised unselected patients, the interpatient variability in trough plasma concentrations (CV\%) observed for imatinib (39.1\%), erlotinib (40.1\%), and sunitinib (29.2\%) was comparable with previously reported data ${ }^{5-7,14,36}$. The observed intrapatient variability was relatively high compared with the interpatient variability (imatinib 35.5\%, erlotinib 35.5\%, and sunitinib 34.5\%); however, it is not known 
whether this was because of patient compliance, concomitant medication, or other factors.

In line with registration studies, patient-related factors age, body weight, and gender did not substantially affect trough plasma concentrations because of large interpatient variabilities ${ }^{10,19,20}$. The influence of female gender on increasing imatinib concentrations was corroborated by previous studies ${ }^{15,38}$. In the study of Widmer et al. $^{38}$, the metabolic clearance of imatinib was decreased in women. The relationship between decreased liver function and increased plasma concentrations has not been confirmed in other studies thus far ${ }^{10,19,20}$. Analogous to these findings, we only observed a tendency toward higher plasma concentrations of imatinib, erlotinib, and sunitinib with increasing ALT and AST activities in serum. The concomitant use of CYP3A4-inducing antiepileptic drugs lead to significantly lower plasma concentrations of imatinib. It has previously been reported that imatinib trough concentrations were reduced 2.9-fold when concurrently used with enzyme inducing antiepileptics ${ }^{39}$. This can explain why a 2-fold imatinib dose increase was not sufficient to reach target trough levels, as observed in a patient using carbamazepine. As expected, a trend of decreased erlotinib plasma concentrations (-28.7\%) with the concomitant use of PPIs or $\mathrm{H}_{2}$-antagonists was observed within our patient cohort. The increased plasma concentrations of sunitinib in combination with PPIs could not be explained by decreased absorption ${ }^{40}$, and it is unknown whether the inhibition of P-gp, BCRP, or other efflux transporters contributes to the increased bioavailability of sunitinib in humans ${ }^{41,42}$.

No prospective clinical trials have been performed to investigate the safety and the effectiveness of TDM for imatinib, erlotinib, and sunitinib therapy. Thus, the ultimate proof that reaching target trough concentrations increases the treatment outcome remains to be awaited. However, when assuming that target trough concentrations are needed for adequate treatment responses, four patient groups with different changes for successful therapy can be defined, as is mentioned in Figure 4.3.3. Hence, patient group 3 (drug concentrations above the target level and no toxicity) is most likely to reach optimal treatment responses. Patients in group 1 (drug concentrations below the target level and no toxicity) may benefit from TDM-guided dose increases. Patients in group 2 (drug concentrations below the target level and toxicity) also need dose adjustments to reach target trough levels; however, TDM would in this case probably not be helpful because toxicities would make dose increases impossible. Patients in group 4 (drug concentrations above the target level and toxicities) risk treatment discontinuation because of these toxicities. Therefore, TDM may support treatment success in these patients by decreasing TKI doses and aiming for TKI concentrations just above the target level. Thus, patients in group 1 and 4 could probably benefit from the implementation of routine TDM, which included $47.3 \%, 65.7 \%$, and $60.0 \%$ of the patient samples for imatinib, erlotinib, and sunitinib in our cohort, respectively. 


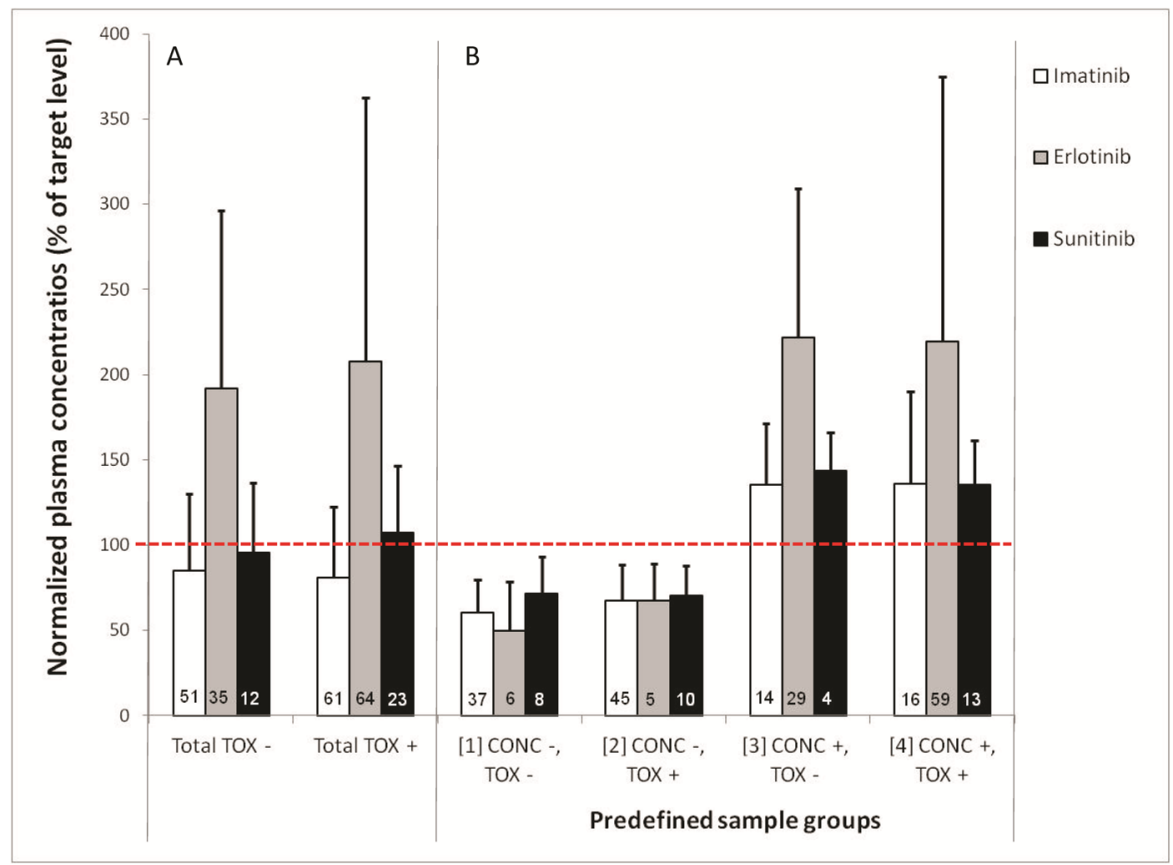

Figure 4.3.3 (A), Mean tyrosine kinase inhibitor plasma concentrations of sample group without the occurrence of toxicity (TOX -) and sample group with the occurrence of toxicity (TOX +); (B), mean plasma concentrations of 4 predefined sample groups, namely, samples of (1) patients with drug concentrations below the target level (CONC -) and without toxicities (TOX -); (2) patients with drug concentrations below the target level (CONC -) and with toxicities (TOX +); (3) patients with drug concentrations above the target level (CONC + ) and without toxicities (TOX -); and (4) patients with drug concentrations above the target level (CONC +) and with toxicities $($ TOX +$)$. Error bars represent the relative standard deviation. Numbers in bars represent the sample number of the specific subgroup of samples.

To identify drugs for which TDM can be an added value in treatment optimisation, different criteria are defined ${ }^{18}$. The anticancer drugs imatinib, erlotinib, and sunitinib fulfill the five most important criteria. These drugs have a narrow therapeutic index (1) with a steep exposure-response relationship $(2)^{4-7,43}$. Additionally, a substantial interpatient variability (3) and relatively small intrapatient variability (4) were observed. Moreover, no easily accessible clinical or laboratory parameter is available that can be used for determining the dosage (5), because no patient- or medication-related factors could completely explain the high interpatient variability in trough concentrations.

In conclusion, around half of the plasma concentrations in the outpatient population seemed to be below the target level. These patients, consequently, could risk treatment failure or development of drug resistance. A large percentage of plasma concentrations below the target level and extremely high concentrations could not be 
explained by patient- or medication-related factors. Accordingly, it is not possible to predict which patients need optimisation of plasma concentrations through TKI dose adjustment. Thus, TDM could play a crucial role in routine cancer care to identify patients that are in need of individual adjusted dosages. 


\section{References}

1. Krause DS, Van Etten RA. Tyrosine kinases as targets for cancer therapy. N Engl J Med. 2005;353(2):172-87.

2. Burris HA 3rd, Hurwitz HI, Dees EC, et al. Phase I safety, pharmacokinetics, and clinical activity study of lapatinib (GW572016), a reversible dual inhibitor of epidermal growth factor receptor tyrosine kinases, in heavily pretreated patients with metastatic carcinomas. J Clin Oncol. 2005;23(23):530513.

3. Calvo E, Rowinsky EK. Effect of epidermal growth factor receptor mutations on the response to epidermal growth factor receptor tyrosine kinase inhibitors: target-based populations for target-based drugs. Clin Lung Cancer. 2004;Suppl1:S35-42.

4. Delbaldo C, Chatelut E, Re M, et al. Pharmacokinetic-pharmacodynamic

relationships of imatinib and its main metabolite in patients with advanced gastrointestinal stromal tumors. Clin Cancer Res. 2006;12(20Pt1):6073-8.

5. Hidalgo M, Siu LL, Nemunaitis J, et al. Phase I and pharmacologic study of OSI-774, an epidermal growth factor receptor tyrosine kinase inhibitor, in patients with advanced solid malignancies. J Clin Oncol. 2001;19(13):3267-79.

6. Houk BE, Bello CL, Poland B, et al. Relationship between exposure to sunitinib and efficacy and tolerability endpoints in patients with cancer: results of a pharmacokinetic/pharmaco-dynamic metaanalysis. Cancer Chemother Pharmacol. 2010;66(2):357-71.

7. Larson RA, Druker BJ, Guilhot F, et al. Imatinib pharmacokinetics and its correlation with response and safety in chronic-phase chronic myeloid leukemia: a subanalysis of the IRIS study. Blood. 2008;111(8):4022-8.

8. Widmer N, Decosterd LA, Leyvraz S, et al. Relationship of imatinib-free plasma levels and target genotype with efficacy and tolerability. Br J Cancer. 2008;98(10):1633-40.

9. Abrams TJ, Murray LJ, Pesenti E, et al. Preclinical evaluation of the tyrosine kinase inhibitor SU11248 as a single agent and in combination with "standard of care" therapeutic agents for the treatment of breast cancer. Mol Cancer Ther. 2003;2(10):1011-21.
10. European Medicinces Agency. Sutent, Summary of Product Characteristics. 2009. Retrieved from https://www.ema.europa.eu/documents/pro duct-information/sutent-epar-productinformation_en.pdf. Accessed July 2012.

11. Faivre S, Delbaldo C, Vera K, et al. Safety, pharmacokinetic, and antitumor activity of SU11248, a novel oral multitarget tyrosine kinase inhibitor, in patients with cancer. J Clin Oncol. 2006;24(1):25-35.

12. Mendel $D B$, Laird $A D, X i n X$, et al. In vivo antitumor activity of SU11248, a novel tyrosine kinase inhibitor targeting vascular endothelial growth factor and platelet-derived growth factor receptors: determination of a pharmacokinetic/pharmacodynamic relationship. Clin Cancer Res. 2003;9(1):32737.

13. Murray LJ, Abrams TJ, Long KR, et al. SU11248 inhibits tumor growth and CSF-1R-dependent osteolysis in an experimental breast cancer bone metastasis model. Clin Exp Metastasis. 2003;20(8):757-66.

14. Picard S, Titier K, Etienne G, et al. Trough imatinib plasma levels are associated with both cytogenetic and molecular responses to standard-dose imatinib in chronic myeloid leukemia. Blood. 2007;109(8):3496-9.

15. Demetri GD, Wang Y, Wehrle E, et al. Imatinib plasma levels are correlated with clinical benefit in patients with unresectable/ metastatic gastrointestinal stromal tumors. J Clin Oncol. 2009;27(19):3141-7.

16. Klümpen $\mathrm{HJ}$, Samer $\mathrm{CF}$, Mathijssen $\mathrm{RH}$, et al. Moving towards dose individualization of tyrosine kinase inhibitors. Cancer Treat Rev. 2011;37(4):251-60.

17. Slaviero KA, Clarke SJ, Rivory LP. Inflammatory response: an unrecognised source of variability in the pharmacokinetics and pharmacodynamics of cancer chemotherapy. Lancet Oncol. 2003;4(4):224-32.

18. de Jonge $M E$, Huitema $A D$, Schellens $J H$, et al. Individualised cancer chemotherapy: strategies and performance of prospective studies on therapeutic drug monitoring with dose adaptation: a review. Clin Pharmacokinet. 2005;44(2):147-73.

19. European Medicinces Agency. Tarceva, Summary of Product Characteristics. 2009. 
Retrieved from https://www.ema.europa.eu/documents/pro duct-information/tarceva-epar-productinformation_en.pdf. Accessed July 2012.

20. European Medicinces Agency. Glivec, Summary of Product Characteristics. 2009. Retrieved from https://www.ema.europa.eu/documents/pro duct-information/glivec-epar-productinformation_en.pdf. Accessed July 2012.

21. Gao B, Yeap S, Clements A, Balakrishnar B, et al. Evidence for Therapeutic Drug Monitoring of Targeted Anticancer Therapies. J Clin Oncol. 2012;30(32):4017-25.

22. Lankheet NA, Steeghs $\mathrm{N}$, Rosing $\mathrm{H}$, et al. Quantification of sunitinib and N-desethyl sunitinib in human EDTA plasma by liquid chromatography coupled with electrospray ionization tandem mass spectrometry: validation and application in routine therapeutic drug monitoring. Ther Drug Monit. 2013;35(2):168-76.

23. Lankheet NAG, Hillebrand MJ, Rosing $\mathrm{H}$, et al. Method development and validation for the quantification of dasatinib, erlotinib, gefitinib, imatinib, lapatinib, nilotinib, sorafenib and sunitinib in human plasma by liquid chromatography coupled with tandem mass spectrometry. Biomed Chromatogr. 2013;27(4):466-76.

24. Wang $\mathrm{Y}$, Chia $\mathrm{YL}$, Nedelman J, et al. A therapeutic drug monitoring algorithm for refining the imatinib trough level obtained at different sampling times. Ther Drug Monit. 2009;31(5):579-84.

25. Naranjo CA, Busto U, Sellers EM, et al. A method for estimating the probability of adverse drug reactions. Clin Pharm Ther. 1981;30(2):239-45.

26. Guilhot F, Hughes TP, Cortes J, et al. Plasma exposure of imatinib and its correlation with clinical response in the Tyrosine Kinase Inhibitor Optimization and Selectivity Trial. Haematologica. 2012;97(5):731-8.

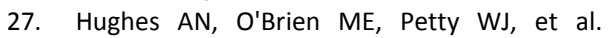
Overcoming CYP1A1/1A2 mediated induction of metabolism by escalating erlotinib dose in current smokers. J Clin Oncol. 2009;27(8):1220-6.

28. Breedveld P, Pluim D, Cipriani G, et al. The effect of Bcrp1 (Abcg2) on the in vivo pharmacokinetics and brain penetration of imatinib mesylate (Gleevec): implications for the use of breast cancer resistance protein and P-glycoprotein inhibitors to enable the brain penetration of imatinib in patients. Cancer Res. 2005;65(7):2577-82.

29. Duong S, Leung M. Should the concomitant use of erlotinib and acid-reducing agents be avoided? The drug interaction between erlotinib and acid-reducing agents. J Oncol Pharm Pract. 2011;17(4):448-52.

30. Haouala A, Widmer N, Duchosal MA, et al. Drug interactions with the tyrosine kinase inhibitors imatinib, dasatinib, and nilotinib. Blood. 2011;117(8):e75-87.

31. Pauli-Magnus $C$, Rekersbrink S, Klotz U, et al. Interaction of omeprazole, lansoprazole and pantoprazole with P-glycoprotein. Naunyn Schmiedebergs Arch Pharmacol. 2001;364(6): 551-7.

32. Solutions ID. NONMEM Users guides. Ellicot City, Maryland, USA; 1989.

33. Kawaguchi T, Hamada A, Hirayama C, et al. Relationship between an effective dose of imatinib, body surface area, and trough drug levels in patients with chronic myeloid leukemia. Int J Hematol. 2009;89(5):642-8.

34. Sohn SK, Oh SJ, Kim BS, et al. Trough plasma imatinib levels are correlated with optimal cytogenetic responses at 6 months after treatment with standard dose of imatinib in newly diagnosed chronic myeloid leukemia. Leuk Lymphoma. 2011;52(6):1024-9.

35. Hartmann JT, Haap M, Kopp HG, et al. Tyrosine kinase inhibitors - a review on pharmacology, metabolism and side effects. Curr Drug Metab. 2009;10(5):470-81.

36. George S, Blay JY, Casali PG, et al. Clinical evaluation of continuous daily dosing of sunitinib malate in patients with advanced gastrointestinal stromal tumour after imatinib failure. Eur J Cancer. 2009;45(11):1959-68.

37. Eechoute K, Fransson MN, Reyners AK, et al. A long-term prospective population

pharmacokinetic study on imatinib plasma concentrations in GIST patients. Clin Cancer Res. 2012;18(20):5780-7.

38. Widmer N, Decosterd LA, Csajka C, et al. Population pharmacokinetics of imatinib and the role of alpha-acid glycoprotein. $\mathrm{Br} \mathrm{J}$ Clin Pharmacol. 2006;62(1):97-112.

39. Pursche S, Schleyer E, von BM, et al. Influence of enzyme-inducing antiepileptic drugs on trough level of imatinib in glioblastoma 
patients. Curr Clin Pharmacol. 2008;3(3):198203.

40. Di Gion $P$, Kanefendt $F$, Lindauer $A$, et al. Clinical pharmacokinetics of tyrosine kinase inhibitors: focus on pyrimidines, pyridines and pyrroles. Clin Pharmacokinet. 2011;50(9): 551-603.

41. Hu S, Chen Z, Franke R, et al. Interaction of the multikinase inhibitors sorafenib and sunitinib with solute carriers and ATP-binding cassette transporters. Clin Cancer Res. 2009;15(19):6062-9.

42. Tang SC, Lagas JS, Lankheet NA, et al. Brain accumulation of sunitinib is restricted by
P-glycoprotein (ABCB1) and breast cancer resistance protein (ABCG2) and can be enhanced by oral elacridar and sunitinib coadministration. Int J Cancer. 2012;130(1): 223-33.

43. Thomas F, Rochaix $\mathrm{P}$, White-Koning $\mathrm{M}$, et al. Population pharmacokinetics of erlotinib and its pharmacokinetic/pharmacodynamic relationships in head and neck squamous cell carcinoma. Eur J Cancer. 2009;45(13):2316-23. 
0 


\section{Chapter}

Summary, general conclusions, and future perspectives 



\section{Summary}

The aim of the first part of the thesis was to investigate the impact of medications for type 2 diabetes mellitus (T2DM) on the risk to develop cancer. The second part of the thesis focused on the implementation of a new anticancer drug after approval by the regulatory authorities and the effectiveness of oncolytic treatment in real-life and per hospital type. In the last part of the thesis we aimed to establish strategies that might help to improve individualised dosing in cancer patients.

In Chapter 1, a general introduction and outline of this thesis are provided.

In Chapter 2.1, we presented the results of a study in which we assessed the association between biguanide (metformin and phenformin) use and colorectal cancer. Previous studies had shown conflicting results, as biguanides were found both to be decreasing and increasing the risk of colorectal cancer in T2DM patients. We conducted a population-based cohort study, using healthcare data from the Danish National database (1996-2007). All patients aged $\geq 18$ years with at least two prescriptions of oral antidiabetic medication $(n=177,281)$ were identified. Control subjects did not have an antidiabetic prescription, and were matched to the antidiabetic drug users in a 3:1 ratio $(n=477,647)$. The primary endpoint was the association between biguanide use and the risk of colorectal cancer. Mean follow-up duration was 5.3 years for the antidiabetic drug users and 6.2 years for the non-diabetic reference group. Current biguanide users had a 1.2-fold increased risk of colorectal cancer as compared to the non-diabetic reference group (95\% confidence interval (Cl) 1.08-1.30). There was, however, no difference in developing colorectal cancer in biguanide users compared with users of other antidiabetic drugs (hazard ratio (HR) 0.95; 95\% Cl 0.87-1.04), who never had used a biguanide. There were also no statistically significant differences between current and past biguanide users versus never users of biguanides. Patients who recently had stopped using biguanides (3-12 months before) seemed to have an increased risk on developing colorectal cancer (HR 1.25; 95\% Cl 1.05-1.50), which was statistically significant when compared with never users of biguanides. Overall, we concluded that biguanide use was not associated with colorectal cancer. T2DM itself was associated with the risk of colorectal cancer.

In Chapter 2.2, we reported the results of another pharmacoepidemiological study in which we investigated the association between incretin use (dipeptidyl peptidase-4 inhibitors (DPP4-Is) and glucagon-like peptide-1 receptor agonists (GLP-1RAs) and pancreatic cancer risk. In this retrospective population-based cohort study, data (20072012) were obtained from the United Kingdom (UK) Clinical Practice Research Datalink (CPRD). Patients with at least one non-insulin antidiabetic drug (NIAD) prescription $(n=210,798)$ and aged $\geq 18$ years during data collection, were matched one-to-one to control patients without T2DM $(n=210,798)$. The primary endpoint was the association 
between incretin use $(n=28,370)$ and the risk of pancreatic cancer. Mean follow-up duration was 4.1 years for incretin users, 3.3 years for other NIAD users and 3.3 years for the non-diabetic reference group. Current NIAD users had a 4.3-fold increased risk (95\% $\mathrm{Cl} 3.49-5.24)$ and current incretin users had a 7.5 -fold increased risk $(95 \% \mathrm{Cl} 5.09$ 11.12) of pancreatic cancer compared with the non-diabetic reference group. There was, however, no statistically significant association between current incretin users and pancreatic cancer as compared to NIAD users who never had used an incretin agent (HR 1.36; 95\% Cl 0.94-1.96). Therefore, we concluded that incretin use was not associated with pancreatic cancer after adjustment for indicators of the severity of the underlying T2DM. T2DM itself was associated with the risk of pancreatic cancer.

The second part of the thesis focused on the implementation of a new anticancer drug after approval by the regulatory authorities (i.e. Food and Drug Administration (FDA) and European Medicines Agency (EMA)) per hospital type and the (determinants of) effectiveness and outcomes of oncolytic treatment in real-life cancer patients.

Previous studies have shown that the implementation of the use of new anti-cancer agents in real-life is often slow and that there is a large variation in the use of treatment options between different hospital types. The implementation and effectiveness of exemestane plus everolimus treatment in patients with advanced breast cancer $(A B C)$ in real-life, shortly after approval of everolimus, is described in Chapter 3.1. In this study, we included all patients with $A B C$ treated with exemestane plus everolimus shortly after approval of everolimus (i.e. the period 2012-2014). The primary endpoint of the study was the progression-free survival (PFS) per hospital type. Data were obtained from the SOutheast Netherlands Advanced BREast cancer (SONABRE) registry. Hospitals were classified as academic, teaching, or non-teaching. PFS and a 12-week conditional PFS (post-hoc) was estimated by the Kaplan-Meier method. The multivariable Cox proportional hazards model was stratified by type of hospital and adjusted for patient, tumour and treatment characteristics. We included 122 patients, comprising 48 patients treated in an academic $(n=1), 56$ in teaching $(n=4)$, and 18 in non-teaching $(\mathrm{n}=2)$ hospitals. The median PFS was 6.3 months $(95 \% \mathrm{Cl} 4.0$ 8.6) overall, and 8.5 months ( $95 \% \mathrm{Cl} 7.7-9.3)$, 4.2 months ( $95 \% \mathrm{Cl} 2.0-6.3)$, and 5.5 (95\% $\mathrm{Cl}$ 4.2-6.7) months for patients treated in academic, teaching and non-teaching hospitals, respectively. The adjusted HR for PFS-events was $1.5(95 \% \mathrm{Cl} 1.0-2.2)$ and 1.0 (95\% $\mathrm{Cl} 0.5-1.9)$ for patients treated at teaching and non-teaching hospitals versus the academic hospital. In contrast, the adjusted HR for 12-week conditional PFS-events was not different between hospital types. We concluded that the median PFS was statistically borderline different between hospital types due to a difference in reported number of PFS-events in the first 12-week treatment period. This seemed to be the result of a different assessment approach. We recommend physicians to broadly share treatment protocols and treatment experience in order to improve the implementation of therapies. 
To assess (determinants of) effectiveness and outcomes of oncolytic treatment in real-life cancer patients, we next studied the impact of dose and simultaneous use of acid reducing agents (ARAs) on the effectiveness of vemurafenib in metastatic BRAF V600 mutated melanoma patients (Chapter 3.2). In this retrospective cohort study, we identified all patients aged $\geq 18$ years with at least one vemurafenib dispensing as firstline treatment for metastatic BRAF V600 mutated melanoma $(n=112)$ using electronic patient records and pharmacy dispensing records of a Dutch academic hospital (2012-2016). The primary endpoint was to assess the association between disease progression of metastatic BRAF V600 mutated melanoma and 1) dose reductions of vemurafenib, and 2) simultaneous use of ARAs. Cox regression analysis was used to estimate the risk of progression with full-dose $(n=64)$ versus reduced-dose vemurafenib $(n=48)$ and with simultaneous use of vemurafenib and ARAs $(n=35)$ versus vemurafenib alone $(n=77)$. Analyses were adjusted for age and sex. The median PFS of the total cohort was 6.0 months $(95 \% \mathrm{Cl} 5.0-6.9)$. There was no association between disease progression and dose reductions of vemurafenib, or with simultaneous use of ARAs (HR 1.12; $95 \% \mathrm{Cl} 0.64-1.61$ and $\mathrm{HR} 1.23 ; 95 \% \mathrm{Cl} 0.53-2.85)$, respectively. In addition, there was no increased risk of progression among patients who used reduced-dose vemurafenib and ARAs versus those receiving full-dose vemurafenib as sole therapy. However, a tendency for disease progression was observed among patients who tolerated full-dose vemurafenib and simultaneously used ARAs versus full-dose vemurafenib alone ( $\mathrm{HR} 2.37 ; 95 \% \mathrm{Cl}$ 0.97-5.76), which increased and became statistically significant in a sensitivity analysis ( $\mathrm{HR} 4.56 ; 95 \% \mathrm{Cl} 1.51-13.75$ ). We concluded that there was no association between the use of vemurafenib in a reduced dose or the simultaneous use of vemurafenib and ARAs and the risk of disease progression. However, patients tolerating full-dose vemurafenib simultaneously with use of ARAs might have an increased risk of progression of metastatic BRAF V600 melanoma. These results need further validation in a larger population.

As we acknowledge that effectiveness in daily practice may differ for various reasons, we focussed in the third part of this thesis on a method that may improve individual treatment outcomes in the near future. Therapeutic drug monitoring (TDM) is a powerful tool that can be used to improve effectiveness and reduce toxicity in reallife patients. However, TDM is currently not routinely used in clinical care of cancer patients mainly due to the lack of evidence from prospective randomised controlled trials (RCTs) that demonstrate its effectiveness. Yet, there is evidence supporting the use of TDM in clinical practice.

Dried blood spots (DBS) sampling can be performed by patients at their home, thereby providing a possibility to measure the trough plasma concentration just before clinical evaluation by the oncologist.

In Chapter 4.1, we describe the results of a study in which we aimed to develop and validate an analytical assay using ultra performance liquid chromatography-tandem mass spectrometry (UPLC-MS/MS) to quantify everolimus in DBS in the oncology 
setting. The assay was validated for standard validation parameters, such as accuracy, precision, matrix effect, and relevant DBS-specific parameters, including hematocrit effect and extensive stability assessment. Everolimus concentrations could be quantified over the range of 3-75 $\mathrm{\mu g} / \mathrm{L}$. The intra- and inter-assay precision and accuracy of the method were shown to be acceptable. The matrix effects appeared to be influenced by the hematocrit effect. The hematocrit effect was tested in a range of $0.20-0.50 \mathrm{~L} / \mathrm{L}$, and were found to be satisfactory at values $\geq 0.25 \mathrm{~L} / \mathrm{L}$. However, at $0.20 \mathrm{~L} / \mathrm{L}$ hematocrit in combination with high everolimus concentrations of 20 and 40 $\mu \mathrm{g} / \mathrm{L}$, the accuracy was $>15 \%$ of the nominal concentration. Everolimus was stable in DBS for at least 80 days at $2-8^{\circ} \mathrm{C}$. We concluded that the everolimus DBS bioanalytical method had been successfully developed and validated.

Subsequently, a clinical validation of the DBS assay was undertaken, of which the results are described in Chapter 4.2. In this observational pharmacokinetic study, patients aged $\geq 18$ years, treated with everolimus for any type tumour were included $(n=22)$. The primary endpoint was to determine the agreement and predictive performance of DBS compared to whole-blood (WB) to measure everolimus concentrations in patients with cancer. Paired DBS and WB samples were collected and analysed using UPLC-MS/MS. Bland-Altman and Passing-Bablok analysis were used to determine method agreement. Limits of clinical relevance were set at a difference of $\pm 25 \%$. Using DBS concentration and Passing-Bablok regression analysis, WB concentrations were predicted. Bland-Altman analysis showed a mean ratio of everolimus WB to DBS concentrations of 0.90 (95\% limits of agreement $0.71-1.08$ ), with $95 \%$ of data points within limits of clinical relevance. Passing-Bablok regression analysis of everolimus DBS versus WB showed no significant constant bias of the intercept (intercept 0.02; 95\% Cl 0.93-1.35), but a small significant proportional bias of the slope (slope $0.89 ; 95 \% \mathrm{Cl}$ 0.76-0.99). Predicted concentrations using DBS concentrations and Passing-Bablok regression analysis showed low bias and imprecision and $90 \%$ of samples had an absolute percentage prediction error of $<20 \%$. We concluded therefore that DBS was a valid method to determine everolimus concentrations in cancer patients.

Tyrosine kinase inhibitors (TKIs) are effective targeted anticancer agents. For several TKIs, plasma concentrations were shown to be related to treatment efficacy and toxicity. Pharmacokinetic variability may, therefore, have important clinical consequences. Plasma concentrations of imatinib, erlotinib, and sunitinib in routine real-life cancer patients were determined, as presented in Chapter 4.3. The primary objectives of this study were to evaluate plasma concentrations of imatinib, erlotinib, and sunitinib, in patients treated in routine clinical practice, and to find possible factors related to plasma concentrations below the target level. We included all patients treated with TKIs as part of their anticancer treatment, comprising 108 patients treated with respectively imatinib $(n=36)$, erlotinib $(n=41)$, and sunitinib $(n=31)$. Randomly timed plasma samples were drawn together with regular laboratory investigations 
during routine outpatient clinic visits. The plasma concentrations of TKIs were determined using high performance liquid chromatography-tandem mass spectrometry (HPLC-MS/MS). Trough concentrations were estimated using the interval between the last dose intake and blood sampling and the mean elimination half-life of the TKIs and compared with target trough concentrations. Outpatient medical records were reviewed to collect data on patient- and medication-related factors that could have contributed to the variation in TKI plasma concentration. In only $26.8 \%, 88.9 \%$ and $51.4 \%$ of the patients treated with respectively imatinib, erlotinib, and sunitinib, the calculated trough plasma concentrations reached the predefined lower boundary of the therapeutic window. The interpatient variability was high, with coefficients of variation of $39.1 \%, 40.1 \%$, and $29.2 \%$ for imatinib, erlotinib, and sunitinib, respectively. It was not possible to predict which patients were at risk of subtherapeutic plasma concentrations based on patient characteristics (e.g. sex, age, body weight) or medication-related factors (e.g. dose, drug-drug interactions). We concluded that almost half of the patients treated in routine clinical practice might be at risk of treatment failure due to subtherapeutic plasma concentrations. Therefore, TDM could play an important role in routine clinical care to identify patients that are in need of dose optimisation.

In Chapter 5 a summary on the thesis is provided, followed by the general discussion and future perspectives.

\section{General discussion}

In RCTs, drugs are tested in the ideal world. Patients with serious comorbidities and patients who use drugs that might interfere with the tested medication are excluded. However, real-life is different. Patients may suffer from chronic diseases such as T2DM or may use chronic medications such as ARAs (even without prescription), and may in addition need therapies for cancer or another life-threatening disease. Some diseases co-occur more frequently than might be expected from incidence and prevalence rates, suggesting a possible causal relationship between the diseases and/or the treatment of these diseases. In addition, comorbidities or co-medication can have an impact on compliance and on pharmacokinetics or-dynamics of a particular oncologic treatment and vice versa.

During the past few years, there has been substantial interest in assessing the risk of colorectal cancer with the use of biguanides and the risk of pancreatic cancer with the use of incretin agents. Indeed, T2DM and cancer are both major threats to human health and these diseases represent respectively the $7^{\text {th }}$ and $2^{\text {nd }}$ cause of death globally $^{1}$. Further, T2DM and cancer are both rapidly increasing in prevalence worldwide. The International Diabetes Federation has projected that the prevalence of T2DM will increase from 382 million in 2013 to 552 million by 2030 and 592 million by 
$2035^{2}$. In addition, the World Health Organization projected global cancer incidence will increase from 15.2 million in 2015 to 21.6 million and to 24.0 million by $2035^{3}$. It is therefore that cancer is a frequent comorbidity in patients with T2DM as is T2DM in patients with cancer. Thus, investigating potential associations between the use of antidiabetic drugs and the risk of cancer is highly relevant.

The notion that biguanides may have beneficial anticancer effects stems mainly from in vitro and experimental animal studies, potentially acting via the indirect activation of AMP-activated protein kinase, which plays a role on activating tumour suppressor genes ${ }^{4,5}$. In addition, biguanide use is known to improve insulin-sensitivity, resulting in a reduction of circulating level of insulin and insulin-like growth factor 1 (IGF-1), that are involved in carcinogenesis through the upregulation of the insulin/ IGF-1 signalling pathway.

The association between biguanides and colorectal cancer has been assessed by numerous studies. Indeed, meta-analyses of observational studies showed a decreased risk of colorectal cancer with the use of metformin ${ }^{5-8}$. But, a crucial factor that could affect the interpretation of the results is that time-related biases were common in many of the studies included in the meta-analyses ${ }^{9}$. Time-related biasses are known to exaggerate the benefits of a drug, resulting in an artificial reduced risk ${ }^{9}$. In addition, the healthy user effect seemed to be present in many studies which could also explain why T2DM patients on metformin had a lower risk of developing colorectal cancer as compared to T2DM patients on other antidiabetic drugs ${ }^{10}$. We performed an observational study with a rigorous design and analysis, aiming to minimize the timerelated biasses and healthy user effect, by using a cohort entry date that was equal to the date of the first anti-hyperglycaemic prescription since start of the study and by performing a 'new user design' (Chapter 2.1) ${ }^{11}$. In this study, we found no protective anticancer effect of biguanides, which was in line with the findings of a meta-analysis of $14 \mathrm{RCTs}^{12}$. Yet, an important limitation of almost all studies (including our observational study) is the relatively short duration of follow-up. Studies have indicated that the transition from large adenoma to carcinoma takes approximately 15 years ${ }^{13}$. However, after stratifying our results by the number of dispensings, we also did not find a beneficial association between biguanide use and colorectal cancer ${ }^{11}$. Therefore, increasing evidence demonstrates against a beneficial association between biguanides and colorectal cancer. In the future it might be interesting to investigate whether the use of biguanides can prevent the recurrence of colorectal cancer.

The association between incretin agents and pancreatic cancer has also been examined extensively. The notion that incretin agents may result in increased pancreatic cancer is emerging from in vitro and in vivo studies, possibly acting via enhancement of $\beta$-cell proliferation, a differentiation of adult stem cells in the ductal pancreatic epithelium, and an inhibition in $\beta$-cell apoptosis ${ }^{14,15}$. Further, within rodent models it was demonstrated that incretin agents could induce pancreatic acinar 
inflammation, which may result in ductal hyperplasia and ultimately in pancreatic cancer $^{16}$.

The hypothesis that the use of incretin agents could be associated with risk of pancreatic cancer has been supported by pharmacovigilance reports ${ }^{17-19}$. However, these studies are highly susceptible to reporting bias, which may overestimate the actual risk. In contrast, the observational studies conducted to date have been conflicting and inconclusive ${ }^{20-23}$. Our real-life population-based cohort study did not find any association between incretin use and pancreatic cancer (Chapter 2.2 ${ }^{24}$. Our findings are in line with the results from recent meta-analyses of RCTs that compared the use of incretin agents to active non-incretin antidiabetic agents or placebo/usual care $^{25-28}$. Again, the duration of follow-up was relatively short to detect a causal effect of incretins on pancreatic cancer. The time span from an initiated pancreatic intraepithelial neoplasia lesion to a parental clone, which will initiate an infiltrating pancreatic carcinoma, is approximately 12 years ${ }^{29}$. Yet, after the stratification of our results by number of dispensings, we also did not find a beneficial association between incretin use and pancreatic cancer ${ }^{24}$. Therefore, increasing evidence demonstrates against an increased association between incretin agents and pancreatic cancer.

Based on the data presented in this thesis and together with data from literature, it can be concluded that both biguanide and incretin use do not have a clinical relevant impact on the risk of colorectal and pancreatic cancer in T2DM patients. It is therefore that both antidiabetic treatment options can be used according to the guidelines in T2DM patients, including those at high risk of colorectal and pancreatic cancer.

We showed further that T2DM itself seemed to be associated with an increased risk of colorectal and pancreatic cancer ${ }^{11,24}$. The increased risk of colorectal and pancreatic cancer in patients with T2DM was also demonstrated in previous meta-analyses ${ }^{30,31}$. In addition, T2DM itself also increased the risk of several other cancers, including liver, endometrium, rectum, breast, and bladder cancer ${ }^{32}$. There are several explanations for the increased risk of cancer in patients with T2DM. First, T2DM can influence the cancer risk by several pathophysiological mechanisms, including hyperglycemia, hyperinsulinemia or chronic inflammation ${ }^{33}$. Second, T2DM and cancer share many risk factors, such as obesity, low physical activity, alcohol consumption and smoking ${ }^{33}$. As a consequence, both people at risk of T2DM and those at risk of cancer are likely to benefit from changing an unhealthy lifestyle into a healthy lifestyle. I, therefore, strongly believe that health care should more focus on prevention.

In patients who already developed cancer, the aim should be to optimise and individualise treatment. This aim can be achieved by understanding the factors that may interfere with achieving the maximum response in patients treated with anticancer agents. Efficacy of new treatment options is often determined within well controlled clinical trials; yet, the effectiveness in the real-life situation may be different. In this thesis a slightly shorter median PFS was observed in real-life than in clinical trials

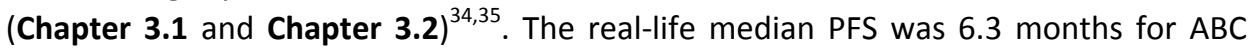


patients treated with exemestane plus everolimus ${ }^{34}$, as compared with the 7.8 months

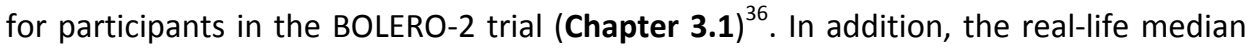
PFS was 6.0 months for metastatic BRAF V600 mutated melanoma patients treated with vemurafenib ${ }^{35}$, as compared with the 6.9 months for patients treated with vemurafenib in the BRIM-3 trial (Chapter 3.2) ${ }^{37}$. Several comments can be made on this issue.

Most importantly, clinical trials use strict in- and exclusion criteria, whereas in reallife patients have generally a worse performance status and more extensive disease. After approval of a new drug, the effectiveness in real-life should be assessed and compared with the reported efficacy from the registration trial(s), to increase awareness on the difference between the two. Also helpful might be to know the proportion of patients treated with a new drug in real-life that would have met the eligibility criteria of the registration trial, to draw conclusions on the generalisability of the results. Ultimately, we should aim to include all patients in registration trials who are potentially able to receive the new anticancer drugs in real-life, in order to increase the generalisability of the RCT results to the daily clinical practice.

Further, in the study on exemestane with everolimus in $A B C$, we observed different numbers on early progression between hospital types ${ }^{34}$. In real-life, the assessment of progressive disease showed to be more pragmatic. It was not always based on imaging, but relative frequently on tumour markers, whereas in the first months of treatment tumour markers may not be a reliable indicator for disease status ${ }^{38}$. Based hereon, we recommend physicians to broadly share treatment protocols and treatment experience.

Lastly, we showed that the effectiveness of vemurafenib in patients with BRAF V600 mutated metastatic melanoma tolerating the full-dose of vemurafenib might be at risk when simultaneously using ARAs ${ }^{35}$. This finding requires prospective validation. Of relevance, the treatment of metastatic BRAF V600 mutated melanoma has undergone several major changes over the past years. Currently, vemurafenib is combined with cobimetinib in patients with metastatic BRAF V600 mutated melanoma, because the coBRIM phase 3 trial demonstrated a significant increase in the median PFS duration when treated with vemurafenib and cobimetinib versus vemurafenib plus placebo ${ }^{39}$. Hence, for new studies on drug-drug interactions between vemurafenib and ARAs, this effect of a potential interaction in the combination should (also) be tested.

From the real-life studies described in this thesis, it can be concluded that both exemestane plus everolimus in $A B C$ and vemurafenib in metastatic BRAF V600 mutated melanoma patients have shown clinical benefit considering the median PFS duration (all patients had progressive disease at start of treatment). This finding was supported by various other real-life studies ${ }^{40-43}$. Knowledge on parameters that are related to treatment outcome is of relevance to inform patients about the effectiveness of the proposed treatment, providing them with a realistic prospect. In addition to effectiveness benefits, patients should also be informed about the tolerability and 
health-related quality of life (HRQoL) to make treatment decisions. Recent studies have shown a maintained HRQoL with both exemestane plus everolimus and cobimetinib in combination with vemurafenib ${ }^{44,45}$. It can, therefore, be concluded that both exemestane plus everolimus and vemurafenib are of value in daily practice to treat patients with respectively $A B C$ and metastatic $B R A F$ V600 mutated melanoma.

At present, there are possibilities to improve the effectiveness of treatment options in individual patients. For example, data on the exposure-response relationships of anticancer agents can be used. The exposure-response relationship of everolimus and vemurafenib has previously been reported ${ }^{43,46-48}$. This information may be used to identify patients who are exposed to supra- or subtherapeutic plasma concentrations, which may be due to drug-drug interactions. An example of patients who may be exposed to subtherapeutic plasma concentrations are BRAF V600 mutated melanoma patients tolerating full-dose vemurafenib and using ARAs simultaneously, since we showed that these patients may be exposed to a potential drug-drug interaction ${ }^{35}$. Of note, information regarding the exposure-response relationship of exemestane plus everolimus and cobimetinib in combination with vemurafenib is currently lacking. Yet, in the era in which combination therapy is the cornerstone of cancer treatment, in an attempt to overcome treatment resistance, it is warranted that the exposure-response relationships of these rational, registered, combination therapies need to be determined.

In Chapter $\mathbf{4}$ of this thesis we have focused on the optimisation of treatment in reallife individual patients with cancer when making use of TDM. We demonstrated that there was a high interpatient variability in exposure of real-life patients $(n=108)$ treated with imatinib, erlotinib, or sunitinib (Chapter 4.3$)^{49}$. In addition, we found that almost $50 \%$ of the calculated trough plasma concentrations were subtherapeutic when using the physicians' prescribed dose of imatinib, erlotinib, or sunitinib ${ }^{49}$. The assessed patient and medication related factors did not entirely explain which patients were at risk of subtherapeutic plasma concentrations. This implied that routine TDM could be valuable for the treatment optimisation of these targeted agents. This finding is supported by previous studies which demonstrated that TDM is a promising strategy for treatment optimisation of imatinib and sunitinib (TDM recommendation viable) and

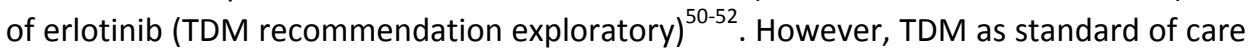
is not performed for any of the targeted agents, including imatinib and sunitinib. As a consequence, fixed dosing of targeted agents is still standard practice. The most important obstacle to overcome the implementation of TDM is the lack of evidence from prospective RCTs that supports the use of pharmacokinetically guided dosing versus fixed dosing ${ }^{50,53}$. Yet, as long as the benefits of pharmaceutical companies to invest in large prospective TDM trials are minimal, these trials may never or only limited be conducted. Fortunately, the Dutch Pharmacology Oncology Group has recently been founded, aiming to determine to what extent TDM actually contributes to a better response and ultimately improved overall survival (http://www.dpog.nl) ${ }^{54}$. 
Another hurdle to overcome before TDM can be implemented in daily practice may be the impractical blood sampling ${ }^{55}$. Currently, patients need to come to the hospital for blood sampling after which the plasma concentrations need to be determined in the laboratory. The feasibility of TDM can potentially be improved when performing TDM with the use of DBS sampling. DBS sampling can be performed by patients at their home and sent by post to the laboratory for analysis. We therefore developed and analytically and clinically validated an everolimus DBS sampling method in the oncology

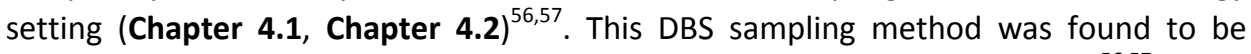
adequate in determining everolimus concentrations in patients with cancer ${ }^{56,57}$. Since the exposure-response relationship of everolimus has already largely been established ${ }^{46,47}$, it is possible that TDM of everolimus may in the future not be that far away.

\section{Future perspectives}

The clinical treatment landscape of cancer treatment is rapidly evolving. Precision medicine is increasingly being used in daily cancer practice, and will be used even more in the near future. It aims to identify which patients benefit the most of a specific intervention based upon the features of the individual and the tumour ${ }^{58}$.

At present, there is a widespread interest in the use of targeted therapy in daily clinical practice and the research setting. Driver mutations and up- or downregulation of relevant cellular pathways can be identified and targeted with rationally designed novel therapeutic agents, irrespective of the initial site and histology of the tumour. This approach has substantially improved the treatment outcomes of many patients with cancer, as illustrated by an improved survival for patients with epidermal growth factor positive (EGFR-positive) lung cancer ${ }^{59}, B R A F$-positive metastatic melanoma ${ }^{60}$ and HER2-positive breast cancer ${ }^{61-63}$. Patients with tumours that do not express a specific molecular profile, do not benefit from the use of such targeted agents ${ }^{64,65}$. It is expected that in the next decade the molecular profile of the tumour will increasingly be used to identify relevant mutations, deletions, or amplifications which will lead to the increasing use of targeted treatments ${ }^{66,67}$.

Hence, we begin to understand the importance of driver mutations and of up- or downregulation of relevant cellular pathways ${ }^{66}$. With this in mind, both European Society for Medical Oncology (ESMO) and American Society of Clinical Oncology (ASCO) have developed a set of criteria a new drug should fulfill before its use will be considered in guidelines ${ }^{68,69}$. For instance, for curative settings, at least $5 \%$ improvement of survival at $\geq 3$-year follow-up and/or improvements in disease-free survival with a hazard ratio of $<0.65$ compared with control without mature survival data is preferable ${ }^{70}$. For non-curative settings if median OS with the standard treatment is $\leq 12$ months, improvements in OS of at least 3 months and a hazard ratio of $\leq 0.65$ 
and/or an increase in 2-year survival alone of at least $10 \%$ is preferable ${ }^{70}$. Last, for single-arm studies in 'orphan diseases' and for diseases with 'high unmet need', a PFS of at least 6 months, an overall response rate (ORR) of at least $60 \%$ and/or an ORR of at least $20 \%$ and a duration of response of at least 9 months is preferable ${ }^{70}$. In the Netherlands, the committee 'BOM' of the Dutch Society of Medical Oncology recommends on the implementation of new drugs, that are EMA approved. Their recommendation is based on the so-called PASQUIL algorithm (i.e. palliative treatment, adjuvant therapy, side effects, quality of life, impact of treatment and level of evidence and cost), using pre-defined minimal efficacy criteria. Yet, the committee BOM is at present not able to provide advice regarding the EMA approved drugs that were based on single-arm studies in 'orphan diseases' and for diseases with 'high unmet need'. Thus far no conditions for recommendations according to the PASQUIL algorithm have been formulated for the drugs that have been EMA approved based on single-arm studies. It is therefore important that these new conditions for recommendations need to be formulated the near future, in order to have the committee BOM act decisively shortly after the approval of new drugs by the EMA.

Yet, besides treating the right patient with the right (targeted) agent, based on the genomic profile of the tumour, it is also important to treat the right patient with the right dose ${ }^{71}$. First, it can be necessary to upfront individualise the dose based on specific pharmacogenetic variants, as is the case for patients with a dihydropyrimidine dehydrogenase deficiency ${ }^{72,73}$. Second, dose individualisation after treatment initiation may be necessary for patients that are exposed to sub- or supratherapeutic concentrations, which may be due to the presence of comorbidities, co-medications, or lifestyle factors ${ }^{71}$. Since, we are not able to predict which patients are exposed to these sub- or supratherapeutic exposure levels, it can be indicated to measure drug exposure in case of severe and unexpected toxicity, a lack of clinical benefit, suspected nonadherence, or (pharmacokinetic) drug-drug interactions ${ }^{54}$. Furthermore, it may also be indicated to determine drug exposure levels in patients engaged in selfmanagement or patients using anticancer drugs for a long period of time, as a way to provide insight in their treatment goals (i.e. to see if the target level has been reached). Since the measurement of drug exposure in oncology is not easy to perform, it is one step forward to determine simple point-of-care tests, such as blood glucose monitoring devices, that can be performed by patients themselves at their homes ${ }^{54}$. In addition, effective clinical tools, such as an app, need to be developed that can aid both physicians and patients in the interpretation and generalisation of an individual dosing advice. Dosing algorithms for future treatment with oral targeted therapies have been developed $^{53}$, which makes dose individualisation of targeted agents not that far away.

Another goal for the future should be to obtain more high-quality real-life data that can be used to perform real-life studies. Real-life studies provide insight in the effectiveness, implementation, (pharmacokinetic) drug-drug interactions and safety of therapeutic anticancer agents in daily practice, enabling to translate data from RCTs to 
real-life patients that more often have comorbidities such as $\mathrm{T}^{\mathrm{D}} \mathrm{DM}^{74}$. Fortunately, the value of real-life data has increasingly been recognised which has led to the routine collection and storage of data in electronic patient records. However, the quality of this data is often poor, due to inconsistencies, data errors, invalid data, missing data and non-standardisation of forms and vocabulary ${ }^{75}$. It is now time to broadly recognise these issues. We should support initiatives that aim to obtain high quality real-life data, such as the multi-year programme 'Registration at the Source ${ }^{76}$, in order to boost the performance of real-life studies in the future.

In conclusion, cancer is a major threat to human health and more awareness should be brought to provide insight in the disease itself and the association between the use of medications such as antidiabetic drugs and the risk of cancer. Real-life studies can improve the outcome of patients with cancer by providing insight in the effectiveness, implementation and drug-drug interactions of novel anticancer agents. The measurement of drug exposure in routine cancer care may be of importance to individualise dosing in order to optimise treatment. It is only by collaboration of a dedicated team of various medical specialists that we can progress in improving treatment outcomes of patients with cancer. 


\section{References}

1. World Health Organization. Home/News/Fact sheets/Detail/Cancer.

Retrieved from http://www.who.int/newsroom/fact-sheets. Accessed July 2018.

2. Guariguata L, Whiting DR, Hambleton I, et al. Global estimates of diabetes prevalence for 2013 and projections for 2035. Diabetes Res Clin Pract. 2014;103(2):137-49.

3. World Health Organization. Global cancer observatory. Cancer Tomorrow. Retrieved from http://gco.iarc.fr/tomorrow/home. Accessed July 2018.

4. Rehman G, Shehzad A, Khan AL, et al. Role of AMP-activated protein kinase in cancer therapy. Arch Pharm (Weinheim). 2014;347(7):457-68.

5. Zhang ZJ, Zheng ZJ, Kan $\mathrm{H}$, et al. Reduced risk of colorectal cancer with metformin therapy in patients with type 2 diabetes: a metaanalysis. Diabetes Care. 2011;34(10):2323-8.

6. He XK, Su TT, Si JM, et al. Metformin Is Associated With Slightly Reduced Risk of Colorectal Cancer and Moderate Survival Benefits in Diabetes Mellitus: A Meta-Analysis. Medicine (Baltimore). 2016;95(7):e2749.

7. Liu F, Yan L, Wang Z, et al. Metformin therapy and risk of colorectal adenomas and colorectal cancer in type 2 diabetes mellitus patients: A systematic review and meta-analysis. Oncotarget. 2017;8(9):16017-26.

8. Singh $\mathrm{S}$, Singh $\mathrm{H}$, Singh PP, et al. Antidiabetic medications and the risk of colorectal cancer in patients with diabetes mellitus: a systematic review and meta-analysis. Cancer Epidemiol Biomarkers Prev. 2013;22(12):225868.

9. Suissa S, Azoulay L. Metformin and the risk of cancer: time-related biases in observational studies. Diabetes Care. 2012;35(12):2665-73.

10. Ioannou GN, Boyko EJ. Metformin and colorectal cancer risk in diabetic patients. Diabetes Care. 2011;34(10):2336-7.

11. Knapen LM, Dittrich ST, de Vries F, et al. Use of biguanides and the risk of colorectal cancer: a register-based cohort study. Curr Drug Saf. 2013;8(5):349-56.

12. Stevens RJ, Ali R, Bankhead CR, et al. Cancer outcomes and all-cause mortality in adults allocated to metformin: systematic review and collaborative meta-analysis of randomised clinical trials. Diabetologia. 2012;55(10):2593603.

13. Jones S, Chen WD, Parmigiani $G$, et al. Comparative lesion sequencing provides insights into tumor evolution. Proc Natl Acad Sci U S A. 2008;105(11):4283-8.

14. Brubaker PL, Drucker DJ. Minireview: Glucagon-like peptides regulate cell proliferation and apoptosis in the pancreas, gut, and central nervous system. Endocrinology. 2004;145(6):2653-9.

15. Spranger J, Gundert-Remy U, Stammschulte T. GLP-1-based therapies: the dilemma of uncertainty. Gastroenterology. 2011;141(1):20-3.

16. Nachnani JS, Bulchandani DG, Nookala A, et al. Biochemical and histological effects of exendin-4 (exenatide) on the rat pancreas. Diabetologia. 2010;53(1):153-9.

17. Raschi E, Piccinni C, Poluzzi E, et al. The association of pancreatitis with antidiabetic drug use: gaining insight through the FDA pharmacovigilance database. Acta Diabetol. 2013;50(4):569-77.

18. Faillie JL, Babai S, Crepin S, et al. Pancreatitis associated with the use of GLP-1 analogs and DPP-4 inhibitors: a case/non-case study from the French Pharmacovigilance Database. Acta Diabetol. 2014;51(3):491-7.

19. Elashoff M, Matveyenko AV, Gier B, et al. Pancreatitis, pancreatic, and thyroid cancer with glucagon-like peptide-1-based therapies. Gastroenterology. 2011;141(1):150-6.

20. Boniol M, Franchi M, Bota M, et al. IncretinBased Therapies and the Short-term Risk of Pancreatic Cancer: Results From Two Retrospective Cohort Studies. Diabetes Care. 2018;41(2):286-92.

21. Azoulay L, Filion KB, Platt RW, et al. Incretin based drugs and the risk of pancreatic cancer: international multicentre cohort study. BMJ. 2016;352:i581.

22. Romley JA, Goldman DP, Solomon M, et al. Exenatide therapy and the risk of pancreatitis and pancreatic cancer in a privately insured population. Diabetes Technol Ther. 2012;14(10):904-11.

23. Funch D, Gydesen $H$, Tornoe $K$, et al. A prospective, claims-based assessment of the risk of pancreatitis and pancreatic cancer with liraglutide compared to other antidiabetic 
drugs. Diabetes Obes Metab. 2014;16(3):2735.

24. Knapen LM, van Dalem J, Keulemans YC et al. Use of incretin agents and risk of pancreatic cancer: a population-based cohort study. Diabetes Obes Metab. 2016;18(3):258-65.

25. Chen H, Zhou X, Chen T, et al. Incretin-Based Therapy and Risk of Pancreatic Cancer in Patients with Type 2 Diabetes Mellitus: A Meta-analysis of Randomized Controlled Trials. Diabetes Ther. 2016;7(4):725-42.

26. Wang $H$, Liu $Y$, Tian $Q$, et al. Incretin-based therapies and risk of pancreatic cancer in patients with type 2 diabetes: A meta-analysis of randomized controlled trials. Diabetes Obes Metab. 2018;20(4):910-20.

27. Monami M, Dicembrini I, Martelli D, et al. Safety of dipeptidyl peptidase-4 inhibitors: a meta-analysis of randomized clinical trials. Curr Med Res Opin. 2011;27Suppl3:57-64.

28. Monami M, Nreu B, Scatena A, et al. Safety issues with glucagon-like peptide-1 receptor agonists (pancreatitis, pancreatic cancer and cholelithiasis): Data from randomized controlled trials. Diabetes Obes Metab. 2017; 19(9):1233-41.

29. Yachida S, Jones S, Bozic I, et al. Distant metastasis occurs late during the genetic evolution of pancreatic cancer. Nature. 2010; 467(7319):1114-7.

30. Larsson SC, Orsini N, Wolk A. Diabetes mellitus and risk of colorectal cancer: a meta-analysis. J Natl Cancer Inst. 2005;97(22):1679-87.

31. Huxley R, Ansary-Moghaddam A, Berrington de Gonzalez A, et al. Type-II diabetes and pancreatic cancer: a meta-analysis of 36 studies. Br J Cancer. 2005;92(11):2076-83.

32. Vigneri $P$, Frasca $F$, Sciacca $L$ et al. Diabetes and cancer. Endocr Relat Cancer. 2009;16(4): 1103-23.

33. Giovannucci E, Harlan DM, Archer MC, et al. Diabetes and cancer: a consensus report. Diabetes Care. 2010;33(7):1674-85.

34. Knapen LM, Geurts SME, De Boer M, et al. A real-life study on everolimus plus exemestane therapy in a cohort of HR-positive, HER2negative postmenopausal advanced breast cancer patients; A study of the SOutheast Netherlands Advanced BREast cancer (SONABRE) Registry. Eur J Cancer. 2018;92: S113.

35. Knapen LM, Koornstra RHT, Driessen JHM, et al. The Impact of Dose and Simultaneous Use of Acid-Reducing Agents on the Effectiveness of Vemurafenib in Metastatic BRAF V600 Mutated Melanoma: a Retrospective Cohort Study. Target Oncol. 2018;13(3):363-370.

36. Yardley DA, Noguchi S, Pritchard $\mathrm{KI}$, et al. Everolimus plus exemestane in postmenopausal patients with $\mathrm{HR}(+)$ breast cancer: BOLERO-2 final progression-free survival analysis. Adv Ther. 2013;30(10):87084.

37. McArthur GA, Chapman PB, Robert C, et al. Safety and efficacy of vemurafenib in BRAF(V600E) and BRAF(V600K) mutationpositive melanoma (BRIM-3): extended follow-up of a phase 3, randomised, openlabel study. Lancet Oncol. 2014;15(3):323-32.

38. Van Poznak C, Somerfield MR, Bast RC, et al. Use of Biomarkers to Guide Decisions on Systemic Therapy for Women With Metastatic Breast Cancer: American Society of Clinical Oncology Clinical Practice Guideline. J Clin Oncol. 2015;33(24):2695-704.

39. Larkin J, Ascierto PA, Dreno B, et al. Combined vemurafenib and cobimetinib in BRAFmutated melanoma. $\mathrm{N}$ Engl J Med. 2014;371(20): 1867-76.

40. Lousberg L, Jerusalem G. Safety, Efficacy, and Patient Acceptability of Everolimus in the Treatment of Breast Cancer. Breast Cancer (Auckl) 2016;10:239-52.

41. Steger GG, Pfeiler G, Petru E, et al. Efficacy and safety of everolimus plus exemestane in $\mathrm{HR}+$, HER2- advanced breast cancer progressing on/after prior endocrine therapy, in routine clinical practice: second interim analysis from STEPAUT. Cancer Research 2017;77(4Suppl):[Abstract P4-22-20].

42. Czirbesz K, Gorka E, Balatoni T, et al. Efficacy of Vemurafenib Treatment in 43 Metastatic Melanoma Patients with BRAF Mutation. Single-Institute Retrospective Analysis, Early Real-Life Survival Data. Pathol Oncol Res. 2019;25(1):45-50.

43. Kramkimel N, Thomas-Schoemann A, Sakji L, et al. Vemurafenib pharmacokinetics and its correlation with efficacy and safety in outpatients with advanced BRAF-mutated melanoma. Target Oncol. 2016;11(1):59-69.

44. Burris HA, 3rd, Lebrun F, Rugo HS, et al. Health-related quality of life of patients with advanced breast cancer treated with everolimus plus exemestane versus placebo plus exemestane in the phase 3 , randomized, 
controlled, BOLERO-2 trial. Cancer. 2013;119(10):1908-15.

45. Dréno $B$, Ascierto $P A$, Atkinson $V$, et al. Health-related quality of life impact of cobimetinib in combination with vemurafenib in patients with advanced or metastatic BRAF(V600) mutation-positive melanoma. Br J Cancer 2018;118(6):777-84.

46. Deppenweiler M, Falkowski S, Saint-Marcoux $F$, et al. Towards therapeutic drug monitoring of everolimus in cancer? Results of an exploratory study of exposure-effect relationship. Pharmacol Res. 2017;121:13844.

47. Ravaud A, Urva SR, Grosch K, et al. Relationship between everolimus exposure and safety and efficacy: meta-analysis of clinical trials in oncology. Eur J Cancer. 2014; 50(3):486-95.

48. Goldwirt L, Chami I, Feugeas JP, et al. Reply to 'Plasma vemurafenib concentrations in advanced BRAFV600mut melanoma patients: impact on tumour response and tolerance' by Funck-Brentano et al. Ann Oncol. 2016;27(2): 363-4.

49. Lankheet NA, Knapen LM, Schellens JH, et al. Plasma concentrations of tyrosine kinase inhibitors imatinib, erlotinib, and sunitinib in routine clinical outpatient cancer care. Ther Drug Monit. 2014;36(3):326-34.

50. Verheijen RB, Yu H, Schellens JHM, et al. Practical Recommendations for Therapeutic Drug Monitoring of Kinase Inhibitors in Oncology. Clin Pharmacol Ther. 2017;102(5):765-76.

51. de Wit D, Guchelaar HJ, den Hartigh J, et al. Individualized dosing of tyrosine kinase inhibitors: are we there yet? Drug Discov Today. 2015;20(1):18-36.

52. Yu H, Steeghs N, Nijenhuis CM, et al. Practical guidelines for therapeutic drug monitoring of anticancer tyrosine kinase inhibitors: focus on the pharmacokinetic targets. Clin Pharmacokinet. 2014;53(4):305-25.

53. Bardin C, Veal G, Paci A, et al. Therapeutic drug monitoring in cancer--are we missing a trick? Eur J Cancer. 2014;50(12):2005-9.

54. Dutch Pharmacology Oncology Group. Retrieved from http://www.dpog.nl. Accessed August 2018.

55. ter Heine R, Huitema ADR, Mathijssen RHJ, et al. 'Therapeutic drug monitoring' van tyrosinekinaseremmers: precisie-geneeskunde nog doeltreffender. Ned Tijdschr Oncol. 2015; 12(7):267-76.

56. Willemsen AECAB, Knapen LM, de Beer YM, et al. Clinical validation study of dried blood spot for determining everolimus concentration in patients with cancer. Eur J Clin Pharmacol. 2018;74(4):465-71.

57. Knapen LM, Beer $Y$, Brüggemann RJM, et al. Development and validation of an analytical method using UPLC-MS/MS to quantify everolimus in dried blood spots in the oncology setting. J Pharm Biomed Anal. 2018; 149:106-13.

58. Yates LR, Seoane J, Le Tourneau C, et al. The European Society for Medical Oncology (ESMO) Precision Medicine Glossary. Ann Oncol. 2018;29(1):30-5.

59. Shepherd FA, Rodrigues Pereira J, Ciuleanu T, et al. Erlotinib in previously treated non-smallcell lung cancer. N Engl J Med. 2005;353(2):123-32.

60. Chapman PB, Hauschild A, Robert C, et al. Improved survival with vemurafenib in melanoma with BRAF V600E mutation. N Engl J Med. 2011;364(26):2507-16.

61. Swain SM, Baselga J, Kim SB, et al. Pertuzumab, trastuzumab, and docetaxel in HER2-positive metastatic breast cancer. N Engl J Med. 2015;372(8):724-34.

62. Blackwell KL, Burstein HJ, Storniolo AM, et al. Overall survival benefit with lapatinib in combination with trastuzumab for patients with human epidermal growth factor receptor 2-positive metastatic breast cancer: final results from the EGF104900 Study. J Clin Oncol. 2012;30(21):2585-92.

63. Verma S, Miles D, Gianni L, et al. Trastuzumab emtansine for HER2-positive advanced breast cancer. N Engl J Med. 2012;367(19):1783-91.

64. Hainsworth JD, Murphy PB, Alemar JR, et al. Use of a multiplexed immunoassay (PRO Onc assay) to detect HER2 abnormalities in circulating tumor cells of women with HER2negative metastatic breast cancer: lack of response to HER2-targeted therapy. Breast Cancer Res Treat. 2016;160(1):41-9.

65. Fehrenbacher L, Cecchini RS, Geyer CE et al. NSABP B-47 (NRG oncology): Phase III randomized trial comparing adjuvant chemotherapy with adriamycin (A) and cyclophosphamide (C) $\rightarrow$ weekly paclitaxel (WP), or docetaxel (T) and $C$ with or without a year of trastuzumab $(H)$ in women with node- 
positive or high-risk node-negative invasive breast cancer (IBC) expressing HER2 staining intensity of IHC $1+$ or $2+$ with negative FISH (HER2-Low IBC). Cancer Research. 2018;GS102.

66. Andre F, Mardis E, Salm M, et al. Prioritizing targets for precision cancer medicine. Ann Oncol. 2014;25(12):2295-303.

67. Bins S, Cirkel GA, Gadellaa-Van Hooijdonk CG, et al. Implementation of a Multicenter Biobanking Collaboration for Next-Generation Sequencing-Based Biomarker Discovery Based on Fresh Frozen Pretreatment Tumor Tissue Biopsies. Oncologist. 2017;22(1):33-40.

68. Cherny NI, Sullivan R, Dafni $U$, et al. A standardised, generic, validated approach to stratify the magnitude of clinical benefit that can be anticipated from anti-cancer therapies: the European Society for Medical Oncology Magnitude of Clinical Benefit Scale (ESMOMCBS). Ann Oncol. 2015;26(8):1547-73.

69. Schnipper LE, Davidson NE, Wollins DS, et al. American Society of Clinical Oncology Statement: A Conceptual Framework to Assess the Value of Cancer Treatment Options. J Clin Oncol. 2015;33(23):2563-77.

70. Cherny NI, Dafni U, Bogaerts J, et al. ESMOMagnitude of Clinical Benefit Scale version 1.1. Ann Oncol. 2017;28(10):2340-66.

71. Mathijssen RH, Sparreboom A, Verweij J. Determining the optimal dose in the development of anticancer agents. Nat Rev Clin Oncol. 2014;11(5):272-81.
72. Meulendijks D, Henricks LM, Sonke GS, et al. Clinical relevance of DPYD variants c.1679T>G, c.1236G >A/HapB3, and c.1601G $>A$ as predictors of severe fluoropyrimidineassociated toxicity: a systematic review and meta-analysis of individual patient data. Lancet Oncol. 2015;16(16):1639-50.

73. Lunenburg C, Henricks LM, Guchelaar HJ, et al. Prospective DPYD genotyping to reduce the risk of fluoropyrimidine-induced severe toxicity: Ready for prime time. Eur J Cancer. 2016;54:40-8.

74. Booth CM, Tannock IF. Randomised controlled trials and population-based observational research: partners in the evolution of medical evidence. Br J Cancer. 2014;110(3):551-5.

75. Chen $\mathrm{H}$, Hailey $\mathrm{D}$, Wang $\mathrm{N}$, et al. A review of data quality assessment methods for public health information systems. Int J Environ Res Public Health. 2014;11(5):5170-207.

76. Nederlandse Federatie van Universitair Medische Centra. Registratie aan de bron. Retrieved from https://www.nfu.nl/programma/registratieaan-de-bron/programma. Accessed August 2018. 

0

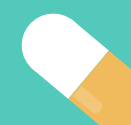

$\theta$

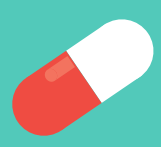

$\theta$

○ 


\section{Appendices}

List of abbreviations Nederlandse samenvatting

Valorisation

List of publications

Dankwoord

Curriculum vitae 



\section{List of abbreviations}

\begin{tabular}{|c|c|}
\hline$A B C$ & Advanced breast cancer \\
\hline ACE & Angiotensin-conversing enzyme \\
\hline $\operatorname{adj}$ & Adjusted \\
\hline ADOPT & A diabetes outcome progression trial \\
\hline ALT & Alanine aminotransferase \\
\hline AMPK & AMP-activated protein kinase \\
\hline ARA & Acid-reducing agents \\
\hline ASCO & American Society of Clinical Oncology \\
\hline AST & Aspartate aminotransferase \\
\hline ATC & Anatomical therapeutic chemical \\
\hline BCRP & Breast cancer resistance protein \\
\hline BID & Twice daily \\
\hline BMI & Body mass index \\
\hline CA $15-3$ & Cancer antigen $15-3$ \\
\hline $\mathrm{Cl}$ & Confidence interval \\
\hline CML & Chronic myeloid leukemia \\
\hline CPRD & Clinical Practice Research Database \\
\hline $\mathrm{C}_{\text {trough }}$ & Trough concentration \\
\hline CV & Coefficient of variation \\
\hline CYP3A4 & Cytochrome P450 enzyme 3A4 \\
\hline DPP4-Is & Dipeptidyl peptidase-4 inhibitors \\
\hline DBS & Dried blood spot \\
\hline EGFR & Epidermal growth factor receptor \\
\hline EMA & European medicines agency \\
\hline eod & Every other day \\
\hline $\mathrm{ERCP}$ & Endoscopic retrograde cholangiopancreatography procedure \\
\hline ESI & Electrospray ionization \\
\hline ESMO & European Society of Medical Oncology \\
\hline FDA & Food and Drug Administration \\
\hline GIST & Gastrointestinal stromal tumour \\
\hline GLP-1 & Glucagon-like peptide-1 \\
\hline GLP-1RAs & Glucagon-like peptide-1 receptor agonists \\
\hline GP & General practitioner \\
\hline HbA1c & Glycosylated hemoglobin type A1c \\
\hline HER2-negative & Human epidermal growth factor receptor 2-negative \\
\hline HPLC-MS/MS & High performance liquid chromatography-tandem mass spectrometry \\
\hline $\mathrm{HR}$ & Hazard ratio \\
\hline HRa & Adjusted hazard ratio \\
\hline HR-positive & Hormone receptor-positive \\
\hline
\end{tabular}




\begin{tabular}{|c|c|}
\hline HRQoL & Health-related quality of life \\
\hline HRT & Hormone replacement therapy \\
\hline ICD & International classification of diseases \\
\hline IGF-1 & Insulin-like growth factor 1 \\
\hline IR & Incidence rate \\
\hline IS & Internal standard \\
\hline KM & Kaplan-Meier \\
\hline LLOQ & Lower limit of quantification \\
\hline LoA & Limits of agreement \\
\hline MAPE & Median absolute percentage prediction error \\
\hline MAPK & Mitogen-activated protein kinase \\
\hline MPE & Median prediction error \\
\hline MPPE & Median percentage prediction error \\
\hline $\mathrm{mRCC}$ & Metastatic renal cell carcinoma \\
\hline mTOR & Mammalian target of rapamycin \\
\hline NIAD & Non-insulin antidiabetic drug \\
\hline no & Number \\
\hline NSAI & Non-steroidal aromatase inhibitor \\
\hline NSAIDs & Non steroidal anti-inflammatory drugs \\
\hline NSCLC & Non-small cell lung cancer \\
\hline OAD & Oral antidiabetic \\
\hline OR & Odds ratio \\
\hline ORR & Overall response rate \\
\hline OS & Overall survival \\
\hline P-gp & P-glycoprotein \\
\hline PI3 & Phosphatidylinositol-3 \\
\hline PFS & Progression-free survival \\
\hline PPIs & Proton pump inhibitors \\
\hline PY & Person-years \\
\hline QC & Quality controls \\
\hline $\mathrm{QCH}$ & Quality control high-level \\
\hline QCL & Quality control low-level \\
\hline QCM & Quality control mid-level \\
\hline QD & Once daily \\
\hline RECORD & $\begin{array}{l}\text { Rosiglitazone evaluated for cardiovascular outcomes and regulation of } \\
\text { glycaemia in diabetes }\end{array}$ \\
\hline RMSE & Root median squared prediction error \\
\hline RCT & Randomized clinical trial \\
\hline RT & Room temperature \\
\hline SD & Standard deviation \\
\hline$S / N$ & Signal-to-noise ratio \\
\hline
\end{tabular}




$\begin{array}{ll}\text { SONABRE } & \text { SOutheast Netherlands Advanced BREast cancer } \\ \text { T2DM } & \text { Type } 2 \text { diabetes mellitus } \\ \text { TDM } & \text { Therapeutic drug monitoring } \\ \text { THIN } & \text { The health information network } \\ \text { TKI } & \text { Tyrosine kinase inhibitor } \\ \text { TZD } & \text { Thiazolidinediones } \\ \text { UK } & \text { United Kingdom } \\ \text { ULOQ } & \text { Upper limit of quantification } \\ \text { UPLC-MS/MS } & \text { Ultra performance liquid chromatography-tandem mass spectrometry } \\ \text { US } & \text { Unites States } \\ \text { WB } & \text { Whole-blood }\end{array}$


Appendices 


\section{Nederlandse samenvatting}

Kanker en type 2 diabetes mellitus (T2DM) zijn veelvoorkomende aandoeningen die een grote bedreiging vormen voor de wereldgezondheid. Wereldwijd is kanker de tweede en T2DM de zevende doodsoorzaak. Tegenwoordig komen er steeds meer en betere behandelingsmogelijkheden voor deze aandoeningen beschikbaar. Echter, kort nadat nieuwe behandelingen op de markt beschikbaar komen, zijn er vaak nog vele onzekerheden. Zo zijn de lange termijn bijwerkingen van nieuwe geneesmiddelen onbekend en is het mogelijk dat een geneesmiddel interacties met andere behandelingen geeft. Daarnaast kunnen de effectiviteit en veiligheid van geneesmiddelen in de dagelijkse praktijk verschillen ten opzichte van de resultaten die worden bereikt in gerandomiseerde interventie onderzoeken. Om de patiënt tot een weloverwogen keuze voor zijn behandeling te laten komen is het van groot belang om onderzoek te doen naar de eerder genoemde aspecten in de dagelijkse praktijk. Ook is het van essentie om te realiseren dat niet elke patiënt dezelfde effectiviteit behaalt met dezelfde therapie. In de dagelijkse praktijk worden alle patiënten met kanker initieel met dezelfde dosis of gelijke dosis per lichaamsomvang behandeld, terwijl hierbij grote verschillen in blootstelling en effectiviteit kan ontstaan. Het is daarom belangrijk dat er onderzoek wordt gedaan naar het optimaliseren van de dosering van een geneesmiddel bij individuele patiënten, zodat idealiter de effectiviteit kan worden verhoogd en de toxiciteit kan worden verlaagd.

Het doel van het eerste gedeelte van dit proefschrift was het bepalen van de associatie tussen het gebruik van bloedglucoseverlagende geneesmiddelen en risico op het ontwikkelen van kanker. Het tweede deel van dit proefschrift focust op de implementatie van nieuwe doelgerichte antikanker geneesmiddelen korte tijd na goedkeuring door regulerende instanties en de effectiviteit van deze geneesmiddelen in de dagelijkse praktijk en per type ziekenhuis. In het derde en laatste gedeelte van het proefschrift was het doel om strategieën te ontwikkelen die kunnen bijdragen aan het optimaliseren van de dosering van oncolytica.

In hoofdstuk 1 wordt een introductie gegeven ten aanzien van de onderwerpen die aan bod komen in dit proefschrift en wordt de opbouw van het proefschrift toegelicht.

In hoofdstuk $\mathbf{2 . 1}$ is een studie uitgevoerd naar de associatie tussen colorectaalkanker en het gebruik van biguanides (metformine en fenformine), aangezien er aanwijzingen waren dat het gebruik van biguanides mogelijk het risico op colorectaalkanker verlaagt. Een retrospectieve populatie-gebaseerde cohortstudie werd uitgevoerd met behulp van een Deense database, waarin informatie is opgenomen over onder andere medische en medicatie gegevens van de gehele Deense bevolking. De studiepopulatie bestond uit alle patiënten ouder dan 18 jaar met minimaal twee recepten voor een oraal bloedglucoseverlagend geneesmiddel binnen de studieperiode (1996-2007). Middels een Cox proportional hazard model werd het risico op colorectaalkanker berekend bij gebruik van biguanides en gecorrigeerd voor 
beïnvloedende factoren ('confounders'). Gebruikers van orale bloedglucoseverlagende geneesmiddelen $(n=177.281)$ werden 1:3 gematcht met een referentie groep die bestond uit mensen zonder T2DM ( $n=477.647)$.

De biguanide gebruikers hadden een 1,2-voudig verhoogd risico op colorectaalkanker in vergelijking met de niet-diabetische referentie groep (95\% betrouwbaarheidsinterval (BI) 1,08-1,30). Echter, de biguanide gebruikers hadden geen verhoogd risico op colorectaalkanker in vergelijking met gebruikers van andere bloedglucoseverlagende geneesmiddelen (gecorrigeerde hazard ratio (HR) 0,95; $95 \% \mathrm{BI}$ 0,87-1,04). In subanalyses werd de invloed van de gebruiksduur en de tijd na het stoppen van biguanides en het risico op colorectaalkanker onderzocht. Beide variabelen hadden geen invloed op de bevindingen, evenals een sensitiviteitsanalyse waarin gebruik gemaakt werd van een 'new user design'.

Samengevat gaf deze studie geen aanleiding om het gebruik van metformine (fenformine is niet meer op de markt) ter preventie van colorectaalkanker in klinische studies verder te onderzoeken.

In hoofdstuk 2.2 wordt de associatie tussen het gebruik van incretines (dipeptidyl peptidase-4 remmers en glucagonachtige peptide-1 receptor agonisten) en het risico op alvleesklierkanker onderzocht, aangezien er aanwijzingen waren dat het gebruik van incretines mogelijk het risico op alvleesklierkanker verhoogt. Er werd gebruik gemaakt van gegevens van de Britse huisartsen database, de 'Clinical Practice Research Datalink' (CPRD), die representatief is voor circa $7 \%$ van de Britse bevolking. De studiepopulatie bestond uit alle patiënten ouder dan 18 jaar met minimaal één recept voor een oraal bloedglucoseverlagend geneesmiddel binnen de studieperiode (juni 2007-augustus 2012). Middels een Cox proportional hazard model werd het risico op alvleesklierkanker berekend bij gebruik van incretines en gecorrigeerd voor beïnvloedende factoren. Gebruikers van orale bloedglucoseverlagende geneesmiddelen $(n=210.798)$ werden 1:1 gematcht met een referentie groep die bestond uit mensen zonder T2DM ( $n=210.798)$.

De gebruikers van incretines $(n=28.370)$ hadden een verhoogd risico op alvleesklierkanker in vergelijking met de niet-diabetische referentie groep (gecorrigeerde HR 7,52; 95\% BI 5,09-11,12). Echter, incretine gebruikers hadden geen verhoogd risico op alvleesklierkanker in vergelijking met gebruikers van andere orale bloedglucoseverlagende geneesmiddelen (gecorrigeerde HR 1,36; 95\% BI 0,94-1,96). In een subanalyse werd de relatie tussen de tijd na stoppen van incretines en het risico op alvleesklierkanker onderzocht. In deze analyse werd een verhoogd risico op alvleesklierkanker gevonden in de recent gestopte gebruikers van incretines (i.e. laatste gebruik 91-180 dagen geleden). Het gevonden toegenomen risico kan het resultaat zijn van protopathische bias ('protopathic bias') of andere vormen van vertekening. Protopathische bias kan optreden als symptomen worden toegeschreven aan het gebruik van een geneesmiddel, terwijl deze symptomen al aanwezig zijn voordat er met het geneesmiddel wordt begonnen. 
Samenvattend gaven de resultaten van deze studie geen aanleiding om te adviseren dat gebruikers van incretines hiermee zouden moeten stoppen vanwege een verhoogd risico op alvleesklierkanker.

In het tweede gedeelte van het proefschrift ligt de focus op de implementatie van nieuwe geneesmiddelen tegen kanker korte tijd na goedkeuring door regulerende instanties. Tevens werd onderzoek gedaan naar (determinanten van de) effectiviteit in de dagelijkse praktijk en per type ziekenhuis.

Eerdere studies hebben aangetoond dat de implementatie van het gebruik van nieuwe geneesmiddelen tegen kanker in de dagelijkse praktijk vaak traag is en dat er een grote variatie is in de volgorde van het gebruik van, en het arsenaal aan behandelingsopties per type ziekenhuis. In hoofdstuk $\mathbf{3 . 1}$ beschrijven we de resultaten van een studie naar de implementatie en effectiviteit van gecombineerd gebruik van exemestaan met everolimus per type ziekenhuis in patiënten met hormoon-receptorpositief, HER2-negatief gemetastaseerd mammacarcinoom. Voor deze studie hebben we gebruik gemaakt van gegevens uit het 'SOutheast Netherlands Advanced BREast cancer' (SONABRE) register, waarin op dit moment gegevens zijn opgenomen van zeven ziekenhuizen en op korte termijn wordt uitgebreid met nogmaals zeven ziekenhuizen in de regio Zuidoost Nederland. Progressievrije overleving was gedefinieerd als tijd tussen startdatum van gebruik van exemestaan met everolimus tot het optreden van progressie. Middels een Cox proportional hazard model werd de progressievrije overleving per type ziekenhuis vanaf de start van behandeling en na 12 weken behandeling met exemestaan met everolimus berekend en gecorrigeerd voor beïnvloedende factoren.

In de studieperiode juli 2012-december 2014 werden 244 patiënten met een diagnose van op afstand gemetastaseerd hormoon-receptor-positief, HER2-negatief mammacarcinoom geregistreerd, waarvan 65,123 , en 56 patiënten werden behandeld in respectievelijk een academisch $(n=1)$, topklinisch $(n=4)$ en algemeen $(n=2)$ ziekenhuis. In totaal werden 122 patiënten behandeld met exemestaan met everolimus, waarvan 48, 56, en 18 patiënten werden behandeld in respectievelijk het academische, de topklinische en algemene ziekenhuizen. Dit onderzoek liet zien dat de mediane progressievrije overleving 6,3 maanden (95\% BI 4,0-8,6) was voor de gehele groep patiënten met gemetastaseerd mammacarcinoom behandeld met exemestaan met everolimus. De mediane progressievrije overleving per type ziekenhuis was respectievelijk 8,5 maanden (95\% BI 7,7-9,3), 4,2 maanden (95\% BI 2,0-6,3) en 5,5 maanden (95\% BI 4,2-6,7) voor patiënten behandeld in het academische, de topklinische en algemene ziekenhuizen. Het risico op progressie was 1,5-voudig verhoogd $(95 \%$ BI 1,0-2,2) voor patiënten behandeld in de topklinische ziekenhuizen en gelijk (gecorrigeerde HR 1,0; 95\% BI 0,5-1,9) voor patiënten behandeld in de algemene ziekenhuizen in vergelijking met het academische ziekenhuis. De gecorrigeerde HR na 12 weken behandeling met exemestaan met everolimus was niet verschillend tussen de type ziekenhuizen. In de eerste 12 weken van behandeling, werd de behandeling met 
exemestaan met everolimus gestopt door vroege progressie in één van de 48 patiënten in het academische ziekenhuis versus negen van de 74 patiënten in de nietacademische ziekenhuizen. In het academische ziekenhuis werd vroege progressie bevestigd door beeldvorming. In de niet-academische ziekenhuizen werd vroege progressie bevestigd door beeldvorming bij twee patiënten en was een verhoging in de tumormarker 15-3 bij vier patiënten, klinische achteruitgang bij twee patiënten en een onbekende oorzaak bij één patiënt tevens reden tot het stoppen van de behandeling.

Concluderend was de mediane progressievrije overleving marginaal significant verschillend tussen de topklinische ziekenhuizen in vergelijking met het academische ziekenhuis, hetgeen mogelijk het resultaat is van een verschil in de wijze van evaluatie en interpretatie van de effectiviteit in de eerste 12 weken van behandeling. Daarnaast werd een verschil in mate van implementatie van everolimus tussen de type ziekenhuizen gezien.

In hoofdstuk 3.2 beschrijven we de resultaten van een studie waarin de impact van de dosering en het gelijktijdig gebruik van maagzuursecretieremmers (protonpompremmers, $\mathrm{H}_{2}$-antagonisten en antacida) op de effectiviteit van vemurafenib in patiënten met gemetastaseerd BRAF V600 gemuteerd melanoom werd onderzocht. Onze hypothese was dat er een verlaagd risico op progressie zou zijn bij patiënten die door toxiciteit behandeld moesten worden met een gereduceerde dosis vemurafenib en een verhoogd risico op progressie bij gelijktijdig gebruik van vemurafenib met maagzuursecretieremmers. Voor deze studie hebben we zowel de aflevergegevens van recepten van de poliklinische apotheek alsmede de gegevens uit de elektronische patiëntendossiers van een academisch ziekenhuis in Nederland gebruikt. Progressievrije overleving was gedefinieerd als tijd tussen startdatum van gebruik van vemurafenib tot het optreden van progressie. Progressie werd gedefinieerd als radiologisch vastgestelde ziekteprogressie of klinische progressie vastgesteld door de behandelaar. Middels een Cox proportional hazard model werd het risico op progressie bij gebruik van de volledige dosering $(n=64)$ versus de gereduceerde dosering vemurafenib $(n=48)$ en bij het gelijktijdig gebruik van vemurafenib en maagzuursecretieremmers $(n=35)$ versus vemurafenib alleen $(n=77)$ berekend en gecorrigeerd voor leeftijd en geslacht.

In totaal waren gedurende de studieperiode $55 \%$ van de patiënten ( $n=112)$ progressief op behandeling met vemurafenib, waarbij de mediane progressievrije overleving 6.0 maanden (95\% BI 5,0-6,9) bedroeg. Er werd geen verlaagd risico op progressie gevonden bij gebruik van een gereduceerde dosering vemurafenib versus het gebruik van de volledige dosering vemurafenib (gecorrigeerde HR 1,12; $95 \% \mathrm{BI}$ $0,64-1,61)$. Ook was er geen verhoogd risico op progressie bij gelijktijdig gebruik van de volledige dosering vemurafenib en maagzuursecretieremmers versus vemurafenib alleen (gecorrigeerde HR 1,23; 95\% BI 0,53-2,85). Tevens vonden we geen toegenomen risico op progressie bij gelijktijdig gebruik van een gereduceerde dosering vemurafenib en maagzuursecretieremmers versus vemurafenib alleen (gecorrigeerde HR 1,00; 
95\% BI 0,45-2,20). Echter, er werd een marginaal significant verhoogd risico op progressie gevonden bij gelijktijdig gebruik van de volledige dosering vemurafenib en maagzuursecretieremmers versus het gebruik van de volledige dosering vemurafenib (gecorrigeerde HR 2,37; 95\% BI 0,97-5,76). Dit risico werd significant verhoogd in een subanalyse, waarbij enkel gebruik werd gemaakt van data uit de aflevergegevens van recepten van de poliklinische apotheek (gecorrigeerde HR 4,56; 95\% BI 1,51-13,75).

Deze resultaten laten zien dat er mogelijk een verhoogd risico is op progressie bij gelijktijdig gebruik van de volledige dosering vemurafenib en maagzuursecretieremmers. Het is noodzakelijk om deze bevinding prospectief te valideren.

Omdat de effectiviteit van orale doelgerichte geneesmiddelen tegen kanker in de dagelijkse praktijk kan verschillen, hebben we in het derde deel van het proefschrift de focus gelegd op een methode die de individuele behandeluitkomsten van patiënten met kanker mogelijk kan verbeteren. Het (regelmatig) meten van plasmaconcentraties is een krachtig instrument dat in de dagelijkse praktijk gebruikt kan worden om de effectiviteit te verbeteren en de toxiciteit te verminderen van de betreffende groep patiënten met kanker. Echter, het aanpassen van de dosering aan de hand van gemeten bloedconcentraties; 'therapeutic drug monitoring' (TDM), wordt hedendaags beperkt routinematig uitgevoerd. De reden is dat het prospectieve bewijs over de toegevoegde waarde onvoldoende is aangetoond. Toch komt er langzamerhand meer bewijs beschikbaar dat het gebruik van TDM de patiëntenzorg in de dagelijkse praktijk ondersteunt.

Bij het gebruik van everolimus als immunosuppressivum wordt in de dagelijkse praktijk de bloedconcentratie regelmatig gecontroleerd, in tegenstelling tot bij het gebruik van everolimus als geneesmiddel tegen kanker. Hoofdstuk 4.1 beschrijft de ontwikkeling en validatie van een analytische droge bloedspot ('dried blood spot', DBS) methode met behulp van 'ultra performance liquid chromatography-tandem mass spectrometry' (UPLC-MS/MS) voor everolimus in patiënten met kanker. Een DBS methode is veelbelovend, omdat deze methode een patiëntvriendelijk alternatief is dat het proces van bloedafname kan vereenvoudigen. Bij deze methode kunnen patiënten zelfstandig in hun eigen huis met een vingerprik een druppel bloed opvangen op een speciaal filterpapier kaartje. Het primaire onderzoeksdoel in onze studie was dan ook om een DBS methode voor everolimus te ontwikkelen en valideren voor het uitvoeren van TDM bij patiënten met kanker. De analytische methode werd gevalideerd conform de richtlijn voor bioanalytische methode ontwikkeling opgesteld door de European Medicines Agency (EMA). Aanvullend op deze richtlijn werden er DBS-specifieke parameters getoetst, zoals het hematocrieteffect, het spotvolume, het terugwinningspercentage en de stabiliteit bij bewaren op DBS kaartjes. De resultaten van de validatie van de DBS bioanalytische methode voldeden aan de eisen opgesteld door de EMA. Echter, bij hematocrietwaarden $<0,25 \mathrm{~L} / \mathrm{L}$ in combinatie met hoge everolimus concentraties van 20 en $40 \mu \mathrm{g} / \mathrm{L}$ voldeed de juistheid niet aan de specificaties. Bij het 
gebruik van de methode in de patiëntenzorg moet hiermee rekening worden gehouden.

Concluderend is de everolimus DBS methode voor patiënten met kanker succesvol ontwikkeld en gevalideerd. Er is echter speciale aandacht nodig voor het uitvoeren van TDM van everolimus bij patiënten met kanker met een hematocrietwaarde van $<0,25$ $\mathrm{L} / \mathrm{L}$ in combinatie met hoge everolimus concentraties van 20 en $40 \mu \mathrm{g} / \mathrm{L}$.

In hoofdstuk 4.2 is er gekeken naar de geschiktheid van de ontwikkelde DBS everolimus methode in patiënten met kanker. Het primaire onderzoeksdoel was om de overeenkomst en predictieve prestatie analyse ('predictive performance') van de everolimus concentraties gemeten in DBS versus de everolimus concentraties gemeten in volbloed te onderzoeken. Met behulp van Bland-Altman grafieken en Passing-Bablok regressie-analyse werd de overeenkomst tussen de everolimus concentraties gemeten in DBS, DBS volbloed (DBS monsters gemaakt van volbloed) en volbloed onderzocht. De limiet voor klinische relevantie werd vastgesteld op een verschil van $\pm 25 \%$, omdat dit verschil zou resulteren in een andere dosering everolimus. Met behulp van de predictieve prestatie analyse werd de overeenkomst tussen everolimus concentraties gemeten in DBS en de volbloed concentraties zoals berekend met behulp van de DBS concentratie en Passing-Bablok regressie-analyse onderzocht.

In totaal werden er 22 patiënten met kanker geïncludeerd in deze studie, waarvan de gepaarde DBS en volbloed monsters van 20 patiënten bruikbaar waren voor analyse. Er werd een goede overeenkomst tussen everolimus concentraties gemeten in DBS en volbloed aangetoond. Bland-Altman grafieken toonden een gemiddelde ratio everolimus volbloed versus DBS van 0,90 , waarbij $95 \%$ van de data binnen de limiet voor klinische relevantie viel. Passing-Bablok regressie-analyse van DBS vergeleken met volbloed toonde geen vaste afwijking (snijpunt 0,02; 95\% BI 0,93-1,35) en een kleine proportionele bias (helling 0,89; 95\% BI 0,76-0,99). De predictieve prestatie analyse toonde een goede overeenkomst tussen everolimus concentraties gemeten in DBS en de volbloed concentraties zoals berekend.

Deze resultaten laten zien dat de everolimus DBS methode geschikt is voor de toepassing van TDM van everolimus bij patiënten met kanker. Deze bevinding is bijzonder waardevol om vroege over- of onderdosering van everolimus vast te stellen, zodat de dosering kort na start van therapie kan worden geoptimaliseerd.

Tyrosine kinase remmers (TKIs) vertegenwoordigen een relatief nieuwe groep aan orale doelgerichte geneesmiddelen tegen kanker. Voor steeds meer TKIs is een relatie aangetoond tussen enerzijds plasmaconcentraties en anderzijds effectiviteit en toxiciteit. In hoofdstuk 4.3 zijn de resultaten weergegeven van een TDM studie naar de plasmaconcentraties van imatinib, erlotinib, en sunitinib van patiënten met kanker in de dagelijkse praktijk. Ook werd onderzocht welke patiënt- en medicatie gerelateerde factoren, zoals gewicht, leeftijd, geslacht en co-medicatie, mogelijk betrokken waren bij het ontstaan van subtherapeutische TKI plasmaconcentraties. De bloedafnames vonden plaats tijdens routinematige controles. De plasmaconcentraties van imatinib, erlotinib, 
en sunitinib werden bepaald met behulp van gevalideerde 'high performance liquid chromatography-tandem mass spectrometry' (HPLC-MS/MS) bepalingsmethodes. De data voor deze studie werden geëxtraheerd uit de elektronische patiëntendossiers van een ziekenhuis in Nederland.

In totaal werden 108 patiënten geïncludeerd. De therapeutische plasmaconcentratie werd in $26,8 \%, 88,9 \%$, en $51,4 \%$ van de patiënten behandeld met respectievelijk imatinib, erlotinib, en sunitinib gehaald. De interpatiënt variabiliteit was hoog, respectievelijk $39,1 \%, 40,1 \%$ en $29,2 \%$ voor imatinib, erlotinib, en sunitinib. De grote variatie in plasmaconcentratie kon niet geheel worden verklaard met de onderzochte patiënt- en medicatie gerelateerde factoren.

Samengevat had bijna de helft van de patiënten subtherapeutische TKI plasmaconcentraties. Het was niet mogelijk om op basis van de onderzochte patiënten medicatie gerelateerde factoren te voorspellen welke patiënten het grootste risico liepen op subtherapeutische plasmaconcentraties. Het (regelmatig) meten van plasmaconcentraties in de dagelijkse praktijk zou daarom een belangrijke rol kunnen spelen in de behandeling met TKIs om te bepalen bij welke patiënten de dosering mogelijk kan worden geoptimaliseerd.

In hoofdstuk $\mathbf{5}$ wordt de samenvatting van dit proefschrift besproken. Tevens worden de uitgevoerde onderzoeken bediscussieerd en in een toekomstig perspectief geplaatst. Hedendaags zijn er toenemende behandelingsmogelijkheden voor patiënten met kanker. De effectiviteit van deze nieuwe behandelingsmogelijkheden hangt samen met een toenemend inzicht in factoren (tumorgenetica) die hebben geresulteerd in het ontstaan van kanker. Daarnaast zijn patiëntkenmerken, zoals het genetisch profiel en comorbiditeit met bijkomend medicatiegebruik, van belang voor het verder individualiseren van de therapie. Door het betrekken van alle hierboven genoemde factoren zijn we steeds beter in staat om niet alleen de juiste patiënt te selecteren voor de juiste behandeling, maar ook de patiënt te behandelen met de juiste dosering. Daarbij is het van belang dat er in de toekomst meer aandacht komt voor het genereren van kwalitatieve hoogstaande data uit de dagelijkse praktijk. Uiteindelijk is het ultieme doel om de uitkomst van de behandeling en de kwaliteit van leven van patiënten met kanker te verbeteren. 
Appendices 


\section{Valorisation}

In this thesis several real-life studies based on patients with type 2 diabetes mellitus (T2DM) using anti-hyperglycaemic agents and patients with cancer using anticancer agents, are presented. Both cancer and T2DM are among the most frequently diagnosed diseases worldwide, whose incidence is only increasing. Both diseases are a major threat to human health, affecting burden of morbidity and leading to mortality in millions of people worldwide. Of the 56.9 million deaths worldwide in 2016, respectively 9.0 and 1.6 million were attributed to the death of people from cancer and $\mathrm{T}^{2} \mathrm{DM}^{1}$. Cancer is characterised by an uncontrolled division of cells which can invade local tissue and metastasize to other organs. T2DM is a chronic disease characterised by high blood glucose levels. Patients with cancer often also suffer from other comorbidities, including T2DM. It is known that about $8-18 \%$ of patients with cancer also have $\mathrm{T}_{2} \mathrm{DM}^{2}$. This thesis provides value for society by gaining real-life insight in 1) safety of the use of anti-hyperglycaemic agents, 2) effectiveness and implementation of anticancer agents, and 3) treatment optimisation of patients with cancer.

In the first part of this thesis, the association between the use of biguanides and the risk of colorectal cancer and the use of incretin-based therapies and the risk of pancreatic cancer was investigated. We found no beneficial effect for the use of biguanides and the risk of colorectal cancer in T2DM patients ${ }^{3}$. In addition, we found no increased risk of pancreatic cancer with the use of incretin agents in T2DM patients ${ }^{4}$. Last, both studies showed that T2DM itself seemed to be associated with an increased risk of colorectal and pancreatic cancer ${ }^{3,4}$. These findings are of societal benefit. First, the finding that our study and others did not show any beneficial effects of biguanide use with the risk of colorectal cancer, supports no need to further investigate this potential inverse association in randomized controlled trials (RCTs). Given that RCTs are expensive and potentially not that effective in investigating (very) rare adverse events that take longer than one year to develop ${ }^{5}$, this study may help to save society both costs and valuable time. In addition, participants of RCTs will not unnecessarily need to be exposed to biguanides. Second, it is valuable for society to learn that the use of incretin agents is not associated with pancreatic cancer. The initial concern of the risk of pancreatic cancer with the use of incretin agents was based on histological findings in human pancreata and spontaneous reports in an adverse event database ${ }^{6,7}$. The European Medicines Agency (EMA) published an assessment report regarding the safety of glucagon-like peptide-1 (GLP-1) based therapies in 2013, stating that large observational studies with minimal residual confounding are needed to investigate this association $^{8}$. To date, our study is one of the largest observational studies that has investigated this association. Residual confounding was minimal as we were able to statistically adjust our analysis for important confounders such as body mass index (BMI), alcohol use and glycosylated hemoglobin type A1c ( $\mathrm{HbA} 1 \mathrm{c})$ value. Thus, the findings from our study provide important reassurance to society that incretin agents 
can probably be safely used as second-line treatment in T2DM patients, including those at high risk of pancreatic cancer. This reassurance is especially valuable given that pancreatic cancer is one of the most lethal worldwide with 5 -year survival rates of stage IV exocrine pancreatic cancer of about $1 \%{ }^{9}$. Third, both studies provide evidence that the disease T2DM itself may be associated with a risk of cancer. There are several explanations for the increased risk of cancer in patients with T2DM. The explanations include pathophysiological mechanisms, such as hyperglycemia, hyperinsulinemia or chronic inflammation, and shared risk factors, such as obesity, low physical activity, alcohol consumption or smoking ${ }^{10}$. As a consequence, both patients at risk of T2DM and cancer are likely to benefit from changing an unhealthy lifestyle into a healthy lifestyle ${ }^{10}$. This learns us that health care should probably focus more on prevention.

Next to safety, we assessed the effectiveness of targeted oncologic therapies in real-life populations of patients with cancer. We determined the median progressionfree survival (PFS) of patients with advanced breast cancer treated with exemestane plus everolimus and of patients with metastatic BRAF V600 mutated melanoma treated with vemurafenib. We found that the real-life PFS was slightly shorter as seen in the RCTs, 6.3 months versus 7.8 months for the patients treated with exemestane plus everolimus $^{11,12}$, and 6.0 months versus 6.9 months for the patients treated with vemurafenib $^{13,14}$. This information may help us to inform patients making treatment decisions. What is more, we found a large difference in median PFS of patients with advanced breast cancer treated with exemestane plus everolimus per type of hospital, which was respectively 8.5 months, 4.2 months and 5.5 months for the patients treated in academic, teaching and non-teaching hospitals ${ }^{11}$. The borderline significantly difference in median PFS between hospital types was possibly the result of a different assessment approach in the first 12-week treatment period ${ }^{11}$. The transparency on the variation in outcome by type of hospital may help to improve the quality of health care, as there is a willingness to share best practices, treatment protocols and treatment experiences. The OncozON (Comprehensive Cancer Network Southeast Netherlands) initiative, which started in 2010, is a good example, where knowledge on best practices in oncological health care is optimally shared. The results from the presented regional real-life studies will be used to improve health care of future patients overall and more specifically in the OncoZON region.

Besides determining the real-life safety and effectiveness of (anticancer) treatment options, we have also investigated methods to measure and potentially optimize the safety and effectiveness of anticancer agents ${ }^{15,16}$. Currently, patients with cancer are increasingly being treated with fixed-dosed anticancer agents that are given orally. Yet, the oral administration route may result in large inter-individual variability in pharmacokinetics and introduces the risk for treatment adherence risks ${ }^{17}$, potentially resulting in less optimal treatment outcomes. Therapeutic drug monitoring (TDM) of anticancer agents has the potential to individualize the dose and thereby potentially optimize treatment outcomes. This thesis includes two studies that describe the 
development of a bioanalytical method together with the analytical and clinical validation of a DBS method to quantify everolimus in patients with cancer ${ }^{15,16}$. The results of these studies showed that the everolimus DBS sampling method in patients with cancer had a sufficient analytical and clinical validity ${ }^{15,16}$, thereby for the first time ever enabling the possibility to perform everolimus DBS sampling in patients with cancer. The benefit for society may especially be present to early recognise patients with cancer that are over- or underexposed and deserve a dose adaption especially to avoid everolimus induced toxicity.

Further, the development and validation of this DBS sampling method also facilitates the miniaturisation of blood collection, since it only requires a blood spotting volume of $30 \mu \mathrm{L}^{18}$. This, in addition to the ability to have patients perform DBS sampling themselves at their homes and sent the samples to the laboratory by regular mail for analysis, potentially eases the use of TDM in oncology ${ }^{18}$. One of the future improvements may be to use DBS sampling to determine all routine health (check-up) parameters along with the (trough) concentration of an anticancer agent, enabling the early detection of deviations or abnormalities. An effective clinical tool may be used to interpret the analysis results and provide the patient and physician with an individual (dosing) advice. Although these ideas are currently both analytically and technologically challenging, it may be achievable when collaborating with the right professionals with the desired expertise.

Last, this thesis consists of real-life (cohort) studies, which are of benefit to society as they provide insight in new, real-life evidence. Real-life evidence cannot be provided by RCTs, as these use well-regulated and controlled settings. Thus, real-life studies are necessary to translate data from RCTs to real-life patients and this thesis has contributed to that. Yet, given that the importance of real-life studies is very clear, it is remarkable that the digital collection and storage of real-life data is not yet available. Data from electronic patient files are not in a standard fashion recorded and also not transferred to a central database. For that reason data in registries, such as the SOutheast Netherlands Advanced BReast cancer (SONABRE) registry, are manually and retrospectively collected by data clerks. The SONABRE registry is quite unique, also showing that it is not easy to build a database on patients with distant recurrence of cancer which is currently not captured in national cancer registries. One of the future improvements should therefore be the collection of relevant real-life data in a consistent, structured and digital (automated) way. By doing so, the SONABRE registry and comparable initiatives can be extended to other regions in the Netherlands and abroad. Further, it should become easier to link data from different databases. Data linkage is not only an important tool to provide complementary information from one data source that will not be captured in the other, but also to enable identifying and reducing potential misclassification. It may therefore be one step forward to link the real-life data from the studies in this thesis to another large health care database. 
In conclusion, this thesis provides essential real-life insight in safety and effectiveness of antidiabetic and anticancer agents in patients with T2DM and cancer. The safety and effectiveness of targeted anticancer agents may be improved by using DBS sampling, which enables to measure drug exposure in routine care to optimise dosing. We hope that the results from this thesis will help stimulate to perform more real-life studies and to generate more real-life evidence. It is only by collaboration of multiple professionals and physicians that we can provide the best possible health care to all real-life patients, regardless of their comorbidities, or type of hospital they are treated in. 


\section{References}

1. World Health Organization. Home/News/Fact sheets/Detail/the-top-10-causes-of-death. Retrieved from: https://www.who.int/newsroom/fact-sheets/detail/the-top-10-causesof-death. Accessed December 2018.

2. Ko C, Chaudhry S. The need for a multidisciplinary approach to cancer care. J Surg Res. 2002;105(1):53-7.

3. Knapen LM, Dittrich ST, de Vries F, et al. Use of biguanides and the risk of colorectal cancer: a register-based cohort study. Curr Drug Saf. 2013;8(5):349-56.

4. Knapen LM, van Dalem J, Keulemans YC, et al. Use of incretin agents and risk of pancreatic cancer: a population-based cohort study. Diabetes Obes Metab. 2016;18(3):258-65.

5. Vandenbroucke JP. When are observational studies as credible as randomised trials? Lancet. 2004;363(9422):1728-31.

6. Butler AE, Campbell-Thompson M, Gurlo $\mathrm{T}$, et al. Marked expansion of exocrine and endocrine pancreas with incretin therapy in humans with increased exocrine pancreas dysplasia and the potential for glucagonproducing neuroendocrine tumors. Diabetes. 2013;62(7):2595-604.

7. Raschi E, Piccinni C, Poluzzi E, et al. The association of pancreatitis with antidiabetic drug use: gaining insight through the FDA pharmacovigilance database. Acta Diabetol. 2013;50(4):569-77.

8. European Medicines Agency. Assessment report for GLP-1 based therapies. 25 July 2013. Retrieved from: https://www.ema.europa.eu/documents/rep ort/assessment-report-article-53-procedureglp-1-based-therapies_en.pdf. Accessed January 2019.

9. American cancer society. Pancreatic Cancer Survival Rates, by Stage. Retrieved from: https://www.cancer.org/cancer/pancreaticcancer/detection-diagnosis-staging/survivalrates.html. Accessed December 2018.

10. Giovannucci E, Harlan DM, Archer MC, et al. Diabetes and cancer: a consensus report. Diabetes Care. 2010;33(7):1674-85.
11. Knapen LM, Geurts SME, De Boer M, et al. A real-life study on everolimus plus exemestane therapy in a cohort of HR-positive, HER2negative postmenopausal advanced breast cancer patients; A study of the SOutheast Netherlands Advanced BREast cancer (SONABRE) Registry. European Journal of Cancer. 2018;92:S3-S113.

12. Yardley DA, Noguchi S, Pritchard $\mathrm{KI}$, et al. Everolimus plus exemestane in postmenopausal patients with $\mathrm{HR}(+)$ breast cancer: BOLERO-2 final progression-free survival analysis. Adv Ther. 2013;30(10): 870-84.

13. Knapen LM, Koornstra RHT, Driessen JHM, et al. The Impact of Dose and Simultaneous Use of Acid-Reducing Agents on the Effectiveness of Vemurafenib in Metastatic BRAF V600 Mutated Melanoma: a Retrospective Cohort Study. Target Oncol. 2018;13(3):363-70.

14. McArthur GA, Chapman PB, Robert C, et al. Safety and efficacy of vemurafenib in BRAF(V600E) and BRAF(V600K) mutationpositive melanoma (BRIM-3): extended follow-up of a phase 3, randomised, openlabel study. Lancet Oncol. 2014;15(3):323-32.

15. Knapen LM, Beer Y, Brüggemann RJM, et al. Development and validation of an analytical method using UPLC-MS/MS to quantify everolimus in dried blood spots in the oncology setting. J Pharm Biomed Anal. 2018; 149:106-13.

16. Willemsen AECAB, Knapen LM, de Beer YM, et al. Clinical validation study of dried blood spot for determining everolimus concentration in patients with cancer. Eur J Clin Pharmacol. 2018;74(4):465-71.

17. Foulon V, Schoffski P, Wolter P. Patient adherence to oral anticancer drugs: an emerging issue in modern oncology. Acta Clin Belg. 2011;66(2):85-96.

18. Bardin C, Veal G, Paci A, et al. Therapeutic drug monitoring in cancer--are we missing a trick? Eur J Cancer. 2014;50(12):2005-9. 
Appendices 


\section{List of publications}

L.M. Knapen, S.T.A.M. Dittrich, F. de Vries, et al. Use of biguanides and the risk of colorectal cancer: a register-based cohort study. Curr Drug Saf. 2013;8(5):349-56.

F. de Vries, M.P. Zeegers, L.M. Knapen, et al. Thiazolidinediones and cancer: duplicate publication bias? Oncologist. 2013;18(10):1147.

N.A. Lankheet, L.M. Knapen, J.H. Schellens, et al. Plasma concentrations of tyrosine kinase inhibitors imatinib, erlotinib, and sunitinib in routine clinical outpatient cancer care. Ther Drug Monit. 2014;36(3):326-34.

L.M. Knapen, N.P. van Erp, H.G.M. Leufkens, et al. Het gebruik van incretines en het risico op alvleesklierkanker: een populatiegebaseerde cohortstudie. Pharm Weekblad. 2016;1:a1627.

L.M. Knapen, J. van Dalem, Y.C. Keulemans, et al. Use of incretin agents and risk of pancreatic cancer: a population-based cohort study. Diabetes Obes Metab. 2016;18(3):258-65.

J.H.M. Driessen, L.M. Knapen, P.P.M.M. Geusens, et al. Fracture risk reduction with use of dipeptidyl peptidase-4 inhibitors: is there immortal time bias? Osteoporos Int. 2017;28(8):2429-2430.

L.M. Knapen, R.G. de Jong, J.H.M. Driessen, et al. Use of incretin agents and risk of acute and chronic pancreatitis: A population-based cohort study. Diabetes Obes Metab. 2017;19(3):401-411.

L.M. Knapen, R.H.T. Koornstra, J.H.M. Driessen, et al. The impact of dose and simultaneous use of acid-reducing agents on the effectiveness of vemurafenib in metastatic BRAF V600 mutated melanoma: A retrospective cohort study. Target Oncol. 2018;13(3):363-370.

A.E.C.A.B. Willemsen, L.M. Knapen, Y.M. de Beer, et al. Clinical validation study of dried blood spot for determining everolimus concentration in patients with cancer. Eur J Clin Pharmacol. 2018;74(4):465-471.

L.M. Knapen, Y.M. de Beer, R.J.M. Brüggemann, et al. Development and validation of an analytical method using UPLC-MS/MS to quantify everolimus in dried blood spots in the oncology setting. J Pharm Biomed Anal. 2018;149:106-113. 
L.M. Knapen, A.M.C. Dingemans, S. Croes. Administration of crizotinib via jejunostomy tube: A case report. OAJ Clin Case Rep. 2018;1:1.

L.M. Knapen, S.M.E. Geurts, K.I.E. Ibragimova, et al. A real-life study on the implementation and effectiveness of exemestane plus everolimus per hospital type in patients with advanced breast cancer. A study of the Southeast Netherlands Advanced Breast Cancer Registry. Breast. 2019;44:46-51. 


\section{Dankwoord}

Dit proefschrift is het resultaat van de samenwerking met velen. Met veel plezier heb ik samen met hen aan mijn proefschrift gewerkt. Graag zou ik in dit dankwoord dan ook een aantal personen in het bijzonder willen bedanken.

Allereerst wil ik de patiënten bedanken die hebben geparticipeerd in de onderzoeken die staan beschreven in dit proefschrift. Zonder hen was het onmogelijk geweest mijn onderzoek te voltooien.

Vervolgens wil ik mijn promotor en co-promotoren, respectievelijk prof. dr. V.C.G. Tjan-Heijnen en dr. S. Croes, dr. F. de Vries en dr. N.P. van Erp, bedanken voor de fijne begeleiding de afgelopen jaren.

Beste Vivianne, ik ben jou zeer dankbaar dat je ervoor gekozen hebt mij als promotor te begeleiden in dit promotietraject. Jouw oneindige kennis en kunde, je onvermoeibare steun en je zorgzaamheid maken dat ik me geen betere promotor kon wensen. Het was een privilege om met je te mogen samenwerken.

Beste Sander, jij was mijn eerste aanspreekpunt. Het was prettig dat ik met alles bij je terecht kon. Ik wil je bedanken voor al het vertrouwen dat je me hebt gegeven, waar ik veel waarde aan heb gehecht en nog hecht.

Beste Frank, door jou heb ik farmacoepidemiologisch onderzoek leren waarderen. Je hebt me veel geleerd over het belang van efficiënt tijdmanagement en het gestructureerd uitvoeren en opschrijven van onderzoek. Mijn dank hiervoor.

Beste Nielka, hoewel op fysieke afstand was jij altijd betrokken bij mijn promotieonderzoek. Jouw deskundigheid op het gebied van farmacokinetische aspecten van orale antikanker geneesmiddelen is van grote waarde geweest voor het succesvol afronden van dit proefschrift. Je enthousiasme en gedrevenheid heb ik als inspirerend ervaren. Bedankt voor al je hulp.

Ik wil de leden van de beoordelingscommissie, prof. dr. N. Schaper, dr. M. Aarts, prof. dr. H.-J. Guchelaar en prof. dr. R. Mathijssen, bedanken voor de geïnvesteerde tijd gedurende het kritisch lezen en beoordelen van mijn proefschrift.

Verder wil ik alle co-auteurs van de verschillende manuscripten bedanken voor jullie kritische blik en waardevolle aanvullingen. In het bijzonder wil ik graag de volgende mensen bedanken. Annemariek Driessen, ik kan je niet genoeg bedanken voor al je hulp. Je bent van enorme waarde geweest voor het uitvoeren van de vele statistische 
analyses, de hulp bij het schrijven van onderzoeksprotocollen en bovenal de mentale support. Khava Ibragimova, bedankt voor al het werk dat je hebt verzet met betrekking tot de SOutheast Netherlands Advanced BREast cancer (SONABRE) register. Veel succes met het afronden van je eigen promotieonderzoek. Yvo de Beer, bedankt voor je hulp bij het ontwikkelen en het uitvoeren van de analytische validatie van de 'dried bloot spot' everolimus methode. Nienke Lankheet, bedankt dat ik tijdens jouw promotietraject samen met je aan de 'nib-studie' heb mogen werken.

Tiny Wouters, bedankt voor de lay-out van het proefschrift.

Lilian Wishaupt, bedankt voor de secretariële ondersteuning bij dit proefschrift.

Alle collega's van het Maastricht Universitair Medisch Centrum, VieCuri Medisch Centrum, Laurentius ziekenhuis en Nij Smellinghe wil ik bedanken voor de fijne samenwerking van de afgelopen jaren.

Dan mijn paranimfen Daan Knapen en Sandra Geurts. Daan, wij delen een passie voor de medische wereld en in het bijzonder de medische oncologie. Wat ben ik trots dat jij als lid van de 'KnapenKids' mijn paranimf wilt zijn. Veel succes met je eigen promotieonderzoek. Sandra, je scherpe analytische blik was verhelderend en heeft me nieuwe inzichten gegeven. Bedankt voor al je hulp bij het uitvoeren van de statistische analyses. Wat fijn dat jij mijn paranimf wilt zijn.

Lieve vrienden, familie en schoonfamilie. Ik wil jullie bedanken voor jullie nimmer afnemende interesse in mijn onderzoek en de nodige afleiding. Ik voel me enorm bevoorrecht om jullie in mijn leven te hebben.

Lieve papa en mama, wat hebben jullie hard gewerkt om ons alles te geven. Bedankt voor jullie onvoorwaardelijke steun en liefde.

Lieve Bas, jij maakt me aan het lachen. Jouw positiviteit is bewonderingswaardig. Ik ben zo trots op je. Bedankt dat je er altijd voor me bent. 


\section{Curriculum vitae}

Lotte Marieke Knapen werd geboren op 11 november 1987 te Blerick. Na het afronden van het tweetalig VWO op het Isendoorn College te Warnsveld in 2006 studeerde zij farmacie aan de Rijksuniversiteit Groningen. Hier haalde zij in 2009 haar bachelor diploma. Tijdens de master farmacie volgde zij in 2011 een onderzoeksstage aan het Dublin Institute of Technology te Dublin. Daarna volgde zij in 2012 een projectstage aan het Slotervaart ziekenhuis/Nederlands Kanker Instituut te Amsterdam. Zij heeft daar een project uitgevoerd over de variatie en determinanten van variatie in bloedspiegels van tyrosine kinase remmers in de oncologie onder begeleiding van prof. dr. A.D.R. Huitema.

$\mathrm{Na}$ het behalen van de master farmacie was zij van 2012 tot 2016 werkzaam als ziekenhuisapotheker in opleiding in het Maastricht Universitair Medisch Centrum+ (MUMC+) te Maastricht in combinatie met het VieCuri Medisch Centrum te Venlo. Gedurende deze periode werden haar taken als ziekenhuisapotheker in opleiding gecombineerd met promotieonderzoek. Haar promotieonderzoek is het resultaat van een samenwerking tussen de afdeling Klinische Farmacie \& Toxicologie onder begeleiding van dr. S. Croes, dr. F. de Vries en dr. N.P. van Erp (Radboud universitair medisch centrum) en de afdeling Medische Oncologie onder begeleiding van prof. dr. V.C.G. Tjan-Heijnen van het MUMC+. Van 2016 tot 2018 heeft zij het promotieonderzoek afgerond in het MUMC+. In 2018 startte zij vervolgens als ziekenhuisapotheker in Nij Smellinghe te Drachten. 
